\title{
Strategic Diastereoselective C1 Functionalization in the Aza- Rocaglamide Scaffold toward Natural Product-Inspired eIF4A Inhibitors
}

\author{
Christian Nilewski*, Theodore D. Michels, Alan X. Xiang, Garrick K. Packard, Paul A. Sprengeler, Boreth Eam, \\ Sarah Fish, Peggy A. Thompson, Christopher J. Wegerski, Justin T. Ernst, Siegfried H. Reich
}

eFFECTOR Therapeutics, 11180 Roselle Street, Suite A, San Diego, CA 92121, United States

\section{Supporting Information}




\section{Table of contents}

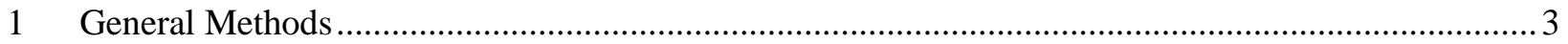

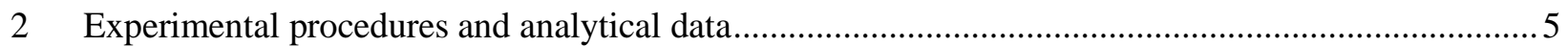

3 Mechanistic investigations of stereoretentive and stereoinvertive displacements ........................... 12

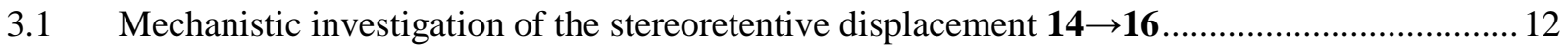

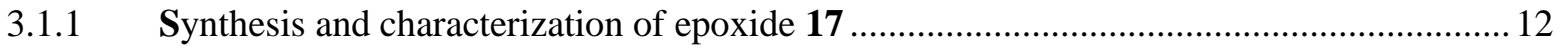

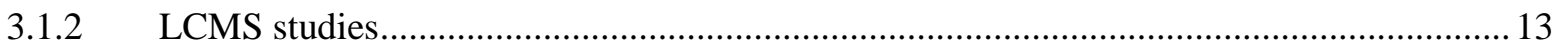

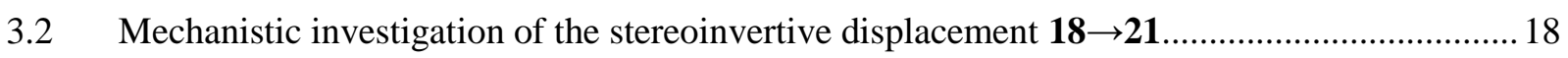

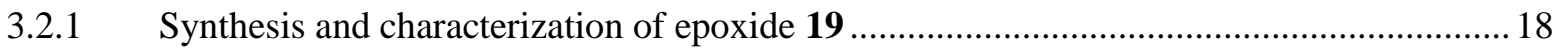

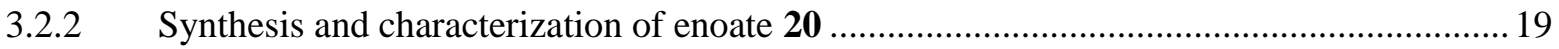

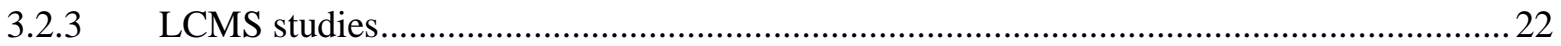

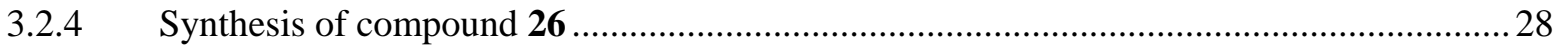

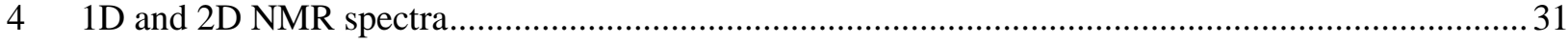

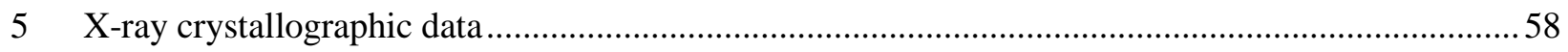

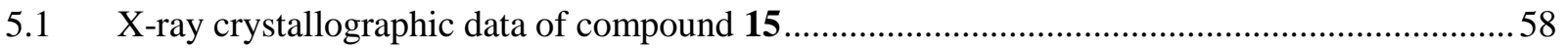

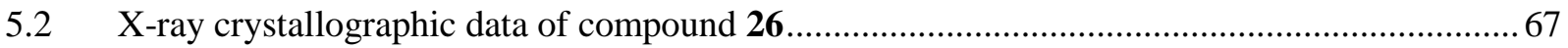

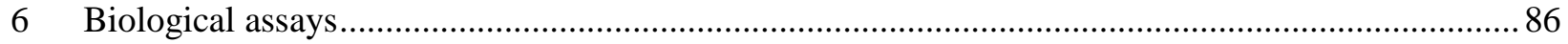

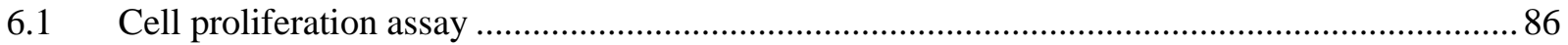

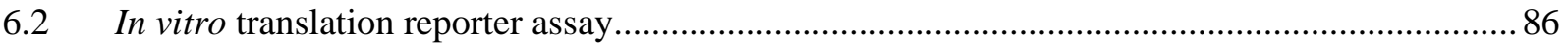




\section{General Methods}

All reagents and solvents were used as purchased from commercial sources unless otherwise noted. Silvestrol ((-)-1) was purchased from MedChemExpress (Monmouth Junction, NJ, USA). Rocaglamide ((-)-2) was synthesized and QCed at Julilant Life Sciences (Uttar Pradesh, India). Cycloheximide was purchased from Sigma-Aldrich (St. Louis, MO).

Microwave reactions were performed with a Biotage Initiator focused beam microwave reactor. Reactions were monitored by TLC (Merck, TLC silica gel 60 F254) or LCMS using the following instruments: Shimadzu LC-10AD liquid chromatograph system equipped with a PE SCIEX API 2000 mass spectrometer, a Shimadzu SCL-10A System controller and SPD-10A UV/Vis detector,

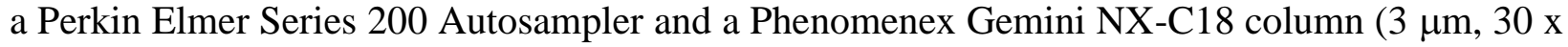
$4.6 \mathrm{~mm}$ ) (mobile phase: 15-95\% MeCN/water + 0.1\% TFA); or an Agilent 1100 series LCMS system equipped with a PE SCIEX API 150EX mass spectrometer and a Phenomenex Gemini NXC18 column ( $3 \mu \mathrm{m}, 30 \times 4.6 \mathrm{~mm})$ (mobile phase: $15-95 \% \mathrm{MeCN} /$ water $+0.1 \%$ TFA).

Flash column chromatography was performed with a Teledyne ISCO CombiFlash Rf200+ system or a Yamazen Smart Flash EPLC W-Prep 2XY system using normal-phase silica columns (230400 mesh).

HPLC purification was performed using a Gilson automated purification system equipped with a Gilson 215 liquid handler and 333 and 334 pumps using Phenomenex Gemini NX-C18 columns $(10 \mu \mathrm{m}, 250 \times 30 \mathrm{~mm}$ or $10 \mu \mathrm{m}, 250 \times 50 \mathrm{~mm})$.

1D $\left({ }^{1} \mathrm{H},{ }^{13} \mathrm{C}\right)$ and 2D NMR (COSY, HSQC, HMBC, NOESY) spectra were recorded on Agilent MR400 and Varian VX 500 spectrometers. Coupling constants $(J)$ are reported in hertz $(\mathrm{Hz})$. Chemical shifts $(\delta)$ of NMR spectra are reported in parts per million (ppm) and are calibrated using residual undeuterated solvent for ${ }^{1} \mathrm{H}$ NMR $\left[{ }^{1} \mathrm{H}=7.26\left(\mathrm{CHCl}_{3}\right), 2.50\left(\mathrm{D}_{5} \mathrm{H}-\mathrm{DMSO}\right), 3.31\right.$ $\left.\left(\mathrm{CD}_{2} \mathrm{HOD}\right) \mathrm{ppm}\right]$ and ${ }^{13} \mathrm{C}$ deuterated solvent for ${ }^{13} \mathrm{C} \mathrm{NMR}\left[77.16\left(\mathrm{CDCl}_{3}\right), 39.52\right.$ (DMSO-d6), $\left.49.00\left(\mathrm{CD}_{3} \mathrm{OD}\right) \mathrm{ppm}\right]$ as an internal reference at $298 \mathrm{~K}^{1}$ The following abbreviations were used to designate the multiplicities: $\mathrm{s}=$ singlet, $\mathrm{d}=$ doublet, $\mathrm{t}=$ triplet, $\mathrm{q}=$ quartet, $\mathrm{m}=$ multiplet, $\mathrm{b}=$ broad.

\footnotetext{
${ }^{1}$ Fulmer, G. R.; Miller, A. J. M.; Sherden, N. H.; Gottlieb, H. E.; Nudelman, A.; Stoltz, B. M.; Bercaw, J. E.; Golberg, K. I. Organometallics 2010, 29, 2176.
} 
Electrospray ionization (ESI) mass spectrometry was performed on PE SCIEX API 2000 and PE SCIEX API 150EX mass spectrometers. High resolution mass spectrometry (HRMS) was performed using a Triple TOF 5600+ mass spectrometer (hybrid quadrupole time-of-flight platform; AB Sciex) connected to a 1290 UHPLC system (Agilent). The mass spectrometer was operated in electrospray positive ionization mode (ESI+). Acquisition was a full scan from $\mathrm{m} / \mathrm{z}$ 100 to 1000 with a pulser frequency of $18.092 \mathrm{kHz}$ and accumulation time of $250 \mathrm{~ms}$. Unless otherwise noted, monoisotopic masses of the $\left[\mathrm{M}+\mathrm{H}^{+}\right]$peaks are reported. All observed isotope patterns were in agreement with calculated isotope patterns. 
2 Experimental procedures and analytical data

Synthetic scheme for the synthesis of compounds 8 and 9:<smiles>CC(=O)c1ncc(Cl)cc1O</smiles>
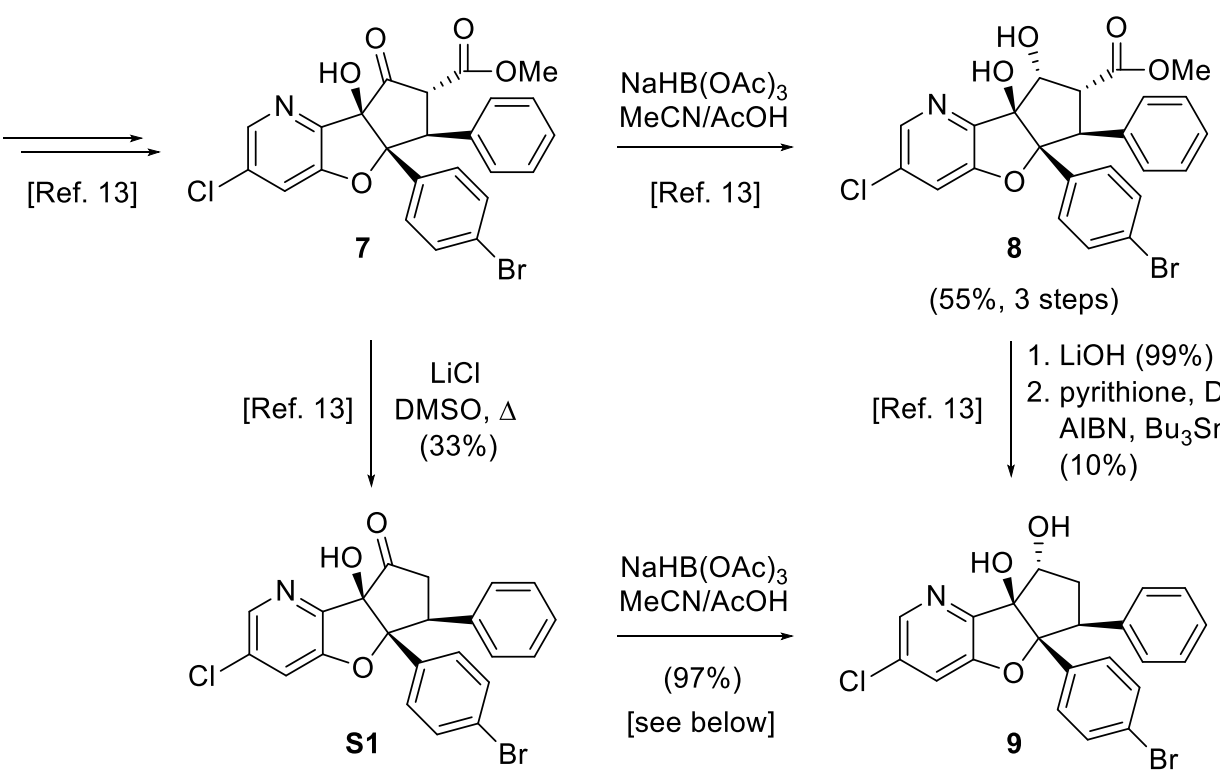

(55\%, 3 steps)
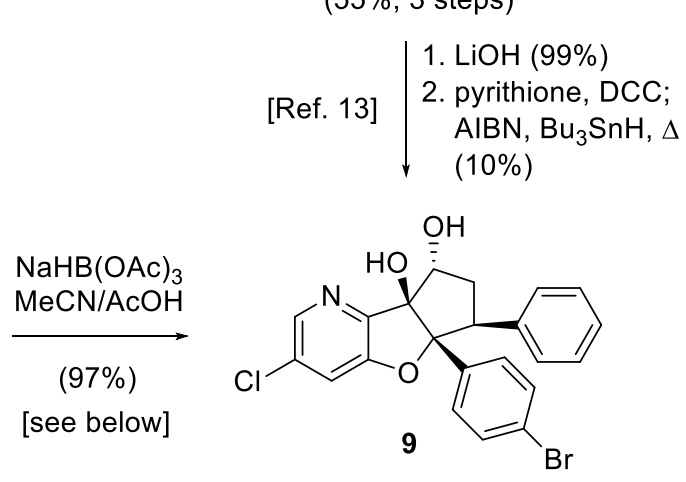

rac-(5aR,6S,8R,8aS)-5a-(4-bromophenyl)-3-chloro-6-phenyl-5a,6,7,8-tetrahydro-8aHcyclopenta[4,5]furo[3,2-b]pyridine-8,8a-diol (9)<smiles>O[C@H]1C[C@@H](c2ccccc2)[C@@]2(c3ccc(Br)cc3)Oc3cc(Cl)cnc3[C@]12O</smiles>

$$
r a c-(5 \mathrm{a} R, 6 S, 8 \mathrm{a} R)-5 \mathrm{a}-(4-\text { bromophenyl)-3-chloro-8a-hydroxy-6- }
$$
phenyl-6,7-dihydrocyclopenta[4,5]furo[1,2-b]pyridin-8-one $(150 \mathrm{mg}, 0.328 \mathrm{mmol})$ was dissolved in acetic acid $(1.4 \mathrm{~mL})$ and $\mathrm{MeCN}(1.4 \mathrm{~mL})$, and sodium triacetoxyborohydride $(0.71 \mathrm{~g}, 3.4$ $\mathrm{mmol}$ ) was added. The mixture was stirred at room temperature. After 20 min water (ca. $10 \mathrm{~mL}$ ) was carefully added. The mixture was extracted ( 3 x ca. $15 \mathrm{~mL}$ DCM), and the combined organic phases were dried $\left(\mathrm{Na}_{2} \mathrm{SO}_{4}\right)$, filtered, and concentrated. Purification by column chromatography $\left(\mathrm{SiO}_{2}, 0-50 \% \mathrm{EtOAC} / \mathrm{Hexane}\right)$ gave product 9 as a white solid. Yield: $146 \mathrm{mg}(0.318 \mathrm{mmol}, 97 \%)$. HRMS (ESI) m/z: $[\mathrm{M}+\mathrm{H}]^{+} \mathrm{Calcd}$ for $\mathrm{C}_{22} \mathrm{H}_{18} \mathrm{BrClNO}_{3}{ }^{+}$458.0153; Found 458.0147; ${ }^{1} \mathbf{H}$ NMR (400 MHz, $\mathbf{d}^{6}$-DMSO) $\delta / \mathrm{ppm}=8.20$ $(\mathrm{d}, J=2.0 \mathrm{~Hz}, 1 \mathrm{H}), 7.65(\mathrm{~d}, J=2.0 \mathrm{~Hz}, 1 \mathrm{H}), 7.23(\mathrm{~d}, J=8.5 \mathrm{~Hz}, 2 \mathrm{H}), 7.15-6.99(\mathrm{~m}, 7 \mathrm{H}), 5.85(\mathrm{~s}$, 1H), $5.12(\mathrm{~b}, 1 \mathrm{H}), 4.48$ (d, $J=4.2 \mathrm{~Hz}, 1 \mathrm{H}), 4.23$ (dd, $J=14.1,6.1 \mathrm{~Hz}, 1 \mathrm{H}), 2.80$ (ddd, $J=13.3$, 13.3, 4.2, 1H), $2.14(\mathrm{dd}, J=13.3,6.1 \mathrm{~Hz}, 1 \mathrm{H}) ;{ }^{13} \mathbf{C}$ NMR (101 MHz, d $\mathbf{d}^{6}$-DMSO) $\delta / \mathrm{ppm}=153.1$, 
$149.7,140.4,138.6,135.8,130.7,129.4,129.3,128.0,127.7,126.1,119.8,116.9,103.1,91.8$, $77.6,53.9,37.7$.

rac-(5a $R, 6 S, 8 R, 8 \mathrm{a} S)-5 \mathrm{a}-(4-b r o m o p h e n y l)-3-c h l o r o-8 a-h y d r o x y-6-p h e n y l-5 a, 7,8,8 \mathrm{a}-$ tetrahydro-6H-cyclopenta[4,5]furo[3,2-b]pyridin-8-yl methanesulfonate (14)

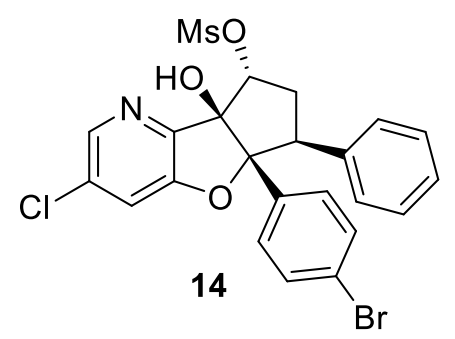

To a solution of $r a c-(5 \mathrm{a} R, 6 S, 8 R, 8 \mathrm{a} S)-5 \mathrm{a}-(4-b r o m o p h e n y l)-3-c h l o r o-$

6-phenyl-7,8-dihydro-6H-cyclopenta[4,5]furo[1,2-b]pyridine-8,8adiol (9) (50 mg, $0.11 \mathrm{mmol})$ in pyridine $(1 \mathrm{~mL})$ was added methanesulfonyl chloride (10 $\mathrm{uL}, 15 \mathrm{mg}, 0.13 \mathrm{mmol})$ and the mixture was stirred at rt. After $22 \mathrm{~h}$ another $3 \mathrm{uL} \mathrm{MsCl}$ added, and the mixture was stirred for two more hours. Then the reaction was quenched with $\mathrm{NH}_{4} \mathrm{Cl}(\mathrm{aq})$ and the mixture was extracted with DCM (3 x ca. $10 \mathrm{~mL})$. The combined organic phases were dried $\left(\mathrm{Na}_{2} \mathrm{SO}_{4}\right)$, filtered and concentrated. Purification by column chromatography $\left(\mathrm{SiO}_{2}, 0-50 \%\right.$ EtOAc/hexane) gave rac-(5aR,6S,8R,8aS)-5a-(4-bromophenyl)-3-chloro-8a-hydroxy-6-phenyl-5a,7,8,8atetrahydro-6H-cyclopenta[4,5]furo[3,2-b]pyridin-8-yl methanesulfonate (14) as a white solid. Yield: $44 \mathrm{mg}(0.082 \mathrm{mmol}, 75 \%)$. HRMS (ESI) m/z: $[\mathrm{M}+\mathrm{H}]^{+}$Calcd for $\mathrm{C}_{23} \mathrm{H}_{20} \mathrm{BrClNO}_{5} \mathrm{~S}^{+}$ 535.9929; Found 535.9916; ${ }^{1}$ H NMR (400 MHz, d6-DMSO) $\delta / \mathrm{ppm}=8.33(\mathrm{~d}, J=2.0,1 \mathrm{H}), 7.82$ $(\mathrm{d}, J=2.0,1 \mathrm{H}), 7.30(\mathrm{~d}, J=8.8,2 \mathrm{H}), 7.17-7.03(\mathrm{~m}, 7 \mathrm{H}), 6.52(\mathrm{~s}, 1 \mathrm{H}), 5.48(\mathrm{dd}, J=5.1,1.3,1 \mathrm{H})$, $4.02(\mathrm{dd}, J=14.3,6.6,1 \mathrm{H}), 3.24(\mathrm{~s}, 3 \mathrm{H}), 3.08(\mathrm{ddd}, J=14.3,14.3,5.1,1 \mathrm{H}), 2.39$ (ddd, $J=14.3$, 6.6, 1.3); ${ }^{13}$ C NMR (101 MHz, d6-DMSO) $\delta / \mathrm{ppm}=152.7,147.3,141.6,137.0,134.3,131.8$, $129.7,129.4,127.9,127.8,126.7,120.5,118.3,102.4,90.0,87.0,53.4,38.1,35.3$.

\section{rac-(5aR,6S,8S,8aR)-5a-(4-bromophenyl)-3-chloro-8a-hydroxy-6-phenyl-5a,7,8,8a-} tetrahydro-6H-cyclopenta[4,5]furo[3,2-b]pyridine-8-carbonitrile (15)

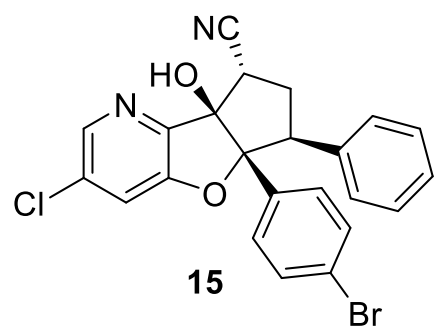

To a solution of $r a c$-[(5aR,6S,8R,8aS)-5a-(4-bromophenyl)-3-chloro8a-hydroxy-6-phenyl-7,8-dihydro-6H-cyclopenta[4,5]furo[1,2b]pyridin-8-yl] methanesulfonate (14) (570 mg, $1.06 \mathrm{mmol})$ in DMSO (10 mL) was added potassium cyanide (142.5 mg, $2.19 \mathrm{mmol})$ and the mixture was stirred at $\mathrm{rt}$ under argon. After $1.5 \mathrm{~h}$ the mixture was diluted with EtOAc and washed brine $(2 \mathrm{x})$, then dried $\left(\mathrm{Na}_{2} \mathrm{SO}_{4}\right)$, filtered and concentrated. Purification by column chromatography $\left(\mathrm{SiO}_{2}, 0-40 \%\right.$ EtOAc/hexane) yielded $496 \mathrm{mg}$ (contained 
ca. 0.55 eq. of EtOAc, $0.965 \mathrm{mmol}, 91 \%$ ) of $\mathbf{1 5}$ as an off-white solid. An analytically pure sample was obtained upon dissolving the material in $\mathrm{CDCl}_{3}$, concentrating and drying under vacuum. HRMS (ESI) m/z: $[\mathrm{M}+\mathrm{H}]^{+}$Calcd for $\mathrm{C}_{23} \mathrm{H}_{17} \mathrm{BrClN}_{2} \mathrm{O}_{2}{ }^{+}$467.0156; Found 467.0145; ${ }^{1} \mathrm{H}$ NMR $\left(400 \mathrm{MHz}, \mathbf{d}^{6}\right.$-DMSO) $\delta / \mathrm{ppm}=8.38(\mathrm{~d}, J=2.0 \mathrm{~Hz}, 1 \mathrm{H}), 7.86(\mathrm{~d}, J=2.0 \mathrm{~Hz}, 1 \mathrm{H}), 7.32(\mathrm{~d}, J=$ $8.8 \mathrm{~Hz}, 2 \mathrm{H}), 7.17-7.08(\mathrm{~m}, 5 \mathrm{H}), 7.04-7.00(\mathrm{~m}, 2 \mathrm{H}), 6.53$ (bs, $1 \mathrm{H}), 4.06(\mathrm{dd}, J=8.8,3.5 \mathrm{~Hz}, 1 \mathrm{H})$, $3.87(\mathrm{dd}, J=12.7,7.0 \mathrm{~Hz}, 1 \mathrm{H}), 3.00(\mathrm{ddd}, J=13.7,12.7,8.8 \mathrm{~Hz}, 1 \mathrm{H}), 2.40(\mathrm{ddd}, J=13.7,7.0,3.5$ $\mathrm{Hz}, 1 \mathrm{H}) ;{ }^{13} \mathbf{C}$ NMR (101 MHz, d $\mathbf{d}^{6}$-DMSO) $\delta / \mathrm{ppm}=151.9,148.3,141.8,137.0,134.0,132.2$, $129.8,129.5,128.0,127.8,126.9,120.7,119.7,118.8,102.4,87.9,54.8,39.7^{*}, 31.9 . *=$ determined by HSQC.

rac-(5aR,6S,8S,8aR)-8-(aminomethyl)-5a-(4-bromophenyl)-3-chloro-6-phenyl-5a,6,7,8tetrahydro-8aH-cyclopenta[4,5]furo[3,2-b]pyridin-8a-ol (TFA salt) (16)

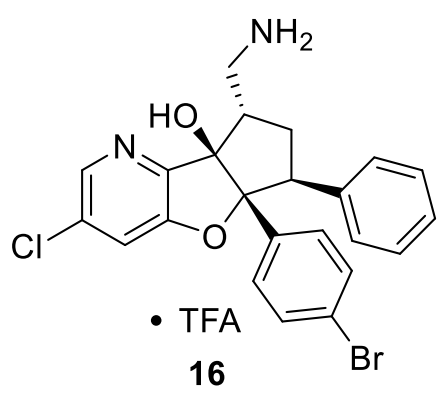

To a solution of $r a c-(5 \mathrm{a} R, 6 S, 8 S, 8 \mathrm{a} R)-5 \mathrm{a}$-(4-bromophenyl)-3-chloro8a-hydroxy-6-phenyl-7,8-dihydro-6H-cyclopenta[4,5]furo-[1,2b]pyridine-8-carbonitrile (15) (20 mg, $0.043 \mathrm{mmol})$ in THF ( $0.5 \mathrm{~mL})$ at $0^{\circ} \mathrm{C}$ was added lithium aluminum hydride $(3.4 \mathrm{mg}, 0.090 \mathrm{mmol}$ ) and the mixture was stirred at rt. After $1 \mathrm{~h} 0.02 \mathrm{~mL}$ water were added at $0^{\circ} \mathrm{C}$, followed by $0.02 \mathrm{~mL} 12.5 \% \mathrm{NaOH}$ (aq) and ca. $60 \mathrm{mg}$ of $\mathrm{Na}_{2} \mathrm{SO}_{4}$. The mixture was stirred for $10 \mathrm{~min}$ at $\mathrm{rt}$, then filtered through celite (rinsed with DCM) and concentrated. The crude product (ca. $19 \mathrm{mg}$, yellow foam) was purified by repeated HPLC $\left(\mathrm{C} 18, \mathrm{MeCN} / \mathrm{H}_{2} \mathrm{O}+0.1 \% \mathrm{TFA}\right)$ to yield $5.3 \mathrm{mg}(0.0091 \mathrm{mmol}, 21 \%)$ of $\mathbf{1 6}$ as a white solid; HRMS (ESI) $\mathrm{m} / \mathrm{z}$ : $[\mathrm{M}+\mathrm{H}]^{+}$Calcd for $\mathrm{C}_{23} \mathrm{H}_{21} \mathrm{BrClN}_{2} \mathrm{O}_{2}{ }^{+} 471.0469$; Found 471.0469; ${ }^{1} \mathrm{H}$ NMR (400 MHz, d'-DMSO, TFA salt) $\delta / \mathrm{ppm}=8.30(\mathrm{~d}, J=2.0 \mathrm{~Hz}, 1 \mathrm{H}), 8.01(\mathrm{~b}, 3 \mathrm{H}), 7.88(\mathrm{~d}, J=2.0 \mathrm{~Hz}, 1 \mathrm{H})$, $7.38(\mathrm{~d}, J=8.8 \mathrm{~Hz}, 2 \mathrm{H}), 7.16-7.08(\mathrm{~m}, 5 \mathrm{H}), 6.96-6.92(\mathrm{~m}, 2 \mathrm{H}), 6.01$ (s, 1H), 3.74 (dd, $J=9.9$, $8.5 \mathrm{~Hz}, 1 \mathrm{H}), 3.56-3.47(\mathrm{~m}, 1 \mathrm{H}), 3.20-3.10(\mathrm{~m}, 1 \mathrm{H}), 3.08-2.97(\mathrm{~m}, 1 \mathrm{H}), 2.60$ (ddd, $J=14.0,9.9$, $9.9 \mathrm{~Hz}, 1 \mathrm{H}), 1.86$ (ddd, $J=14.0,8.5,6.2,1 \mathrm{H}) ;{ }^{13} \mathbf{C}$ NMR (101 MHz, d6 - -DMSO, TFA salt) $\delta$ / $\mathrm{ppm}=152.5,149.5,140.9,138.2,134.2,132.1,129.9,129.4,128.1,127.8,126.7,120.5,119.2$, 103.0, 87.5, 52.7, 46.1, 41.1, 29.7. 

sulfonyl)-oxy)-6-phenyl-5a,7,8,8a-tetrahydro-6H-cyclopenta[4,5]furo[3,2-b]pyridine-7carboxylate (18)

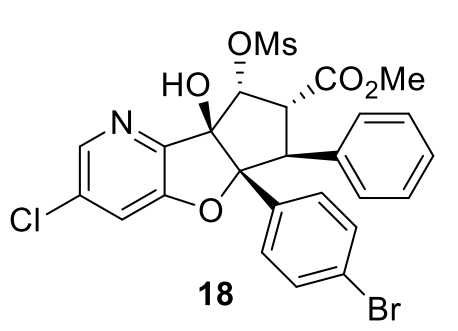

To a solution of rac-methyl $(5 \mathrm{a} R, 6 S, 7 R, 8 R, 8 \mathrm{a} S)-5 \mathrm{a}-(4-$ bromophenyl)-3-chloro-8,8a-dihydroxy-6-phenyl-7,8-dihydro-6Hcyclopenta[4,5]furo[1,2-b]pyridine-7-carboxylate $(\mathbf{8})(800 \mathrm{mg}, 1.55$ mmol) in pyridine $(15 \mathrm{~mL})$ was added methanesulfonyl chloride $(0.15 \mathrm{~mL}, 0.22 \mathrm{~g}, 1.9 \mathrm{mmol})$ and the mixture was stirred at rt. After $23.5 \mathrm{~h}$ another $0.08 \mathrm{~mL}(0.1 \mathrm{~g}, 1 \mathrm{mmol})$ methanesulfonyl chloride added, and the mixture was stirred for another $3.5 \mathrm{~h}$. Another $0.1 \mathrm{~mL}(0.1 \mathrm{~g}, 1 \mathrm{mmol})$ methanesulfonyl chloride were added, and the mixture was stirred for ca. another $1 \mathrm{~h}$. Then the reaction was quenched with $\mathrm{NH}_{4} \mathrm{Cl}$ (aq) and the mixture was extracted with DCM thrice. The combined organic phases were dried $\left(\mathrm{Na}_{2} \mathrm{SO}_{4}\right)$, filtered and concentrated. The crude product was purified by column chromatography $\left(\mathrm{SiO}_{2}, 0-50 \%\right.$ ethyl acetate/hexane) to yield $775 \mathrm{mg}(1.30 \mathrm{mmol}, 84 \%)$ of $\mathbf{1 8}$ as a yellowish foam; HRMS (ESI) m/z: $[\mathrm{M}+\mathrm{H}]^{+}$Calcd for $\mathrm{C}_{25} \mathrm{H}_{22} \mathrm{BrClNO}_{7} \mathrm{~S}^{+}$593.9983; Found 593.9960; ${ }^{1} \mathbf{H}$ NMR

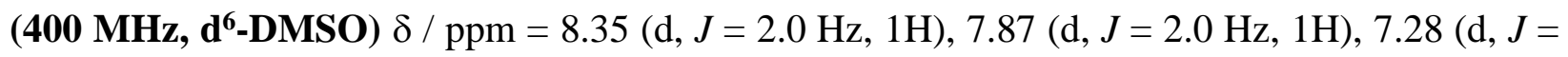
$8.6 \mathrm{~Hz}, 2 \mathrm{H}), 7.13$ (d, J = 8.6 Hz, 2H), 7.13-7.04 (m, 3H), 7.00-6.96 (m, 2H), 6.76 (s, 1H), 5.69 (d, $J=6.1 \mathrm{~Hz}, 1 \mathrm{H}), 4.46(\mathrm{dd}, J=14.5,6.1 \mathrm{~Hz}, 1 \mathrm{H}), 4.26(\mathrm{~d}, J=14.5 \mathrm{~Hz}, 1 \mathrm{H}), 3.57$ (s, 3H), 3.15 (s, 3H); ${ }^{13}$ C NMR (101 MHz, d6-DMSO) $\delta / \mathrm{ppm}=168.3,152.9,147.0,141.7,135.7,133.5,132.2$, $129.7,129.7,127.9,127.7,126.9,120.6,118.7,101.1,89.2,86.4,55.4,52.1,49.1,38.6$.

rac-methyl (5a $R, 6 S, 7 R, 8 R, 8 \mathrm{a} R)-5 \mathrm{a}-(4-b r o m o p h e n y l)-3-c h l o r o-8-c y a n o-8 a-h y d r o x y-6-$ phenyl-5a,7,8,8a-tetrahydro-6H-cyclopenta[4,5]furo[3,2-b]pyridine-7-carboxylate (21)

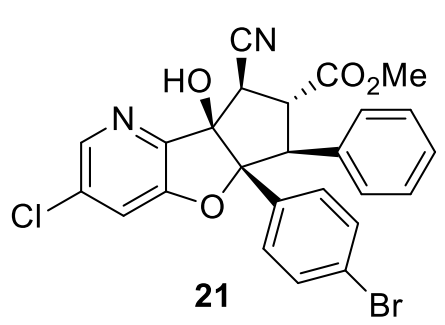

To a solution of rac-methyl $(5 \mathrm{a} R, 6 \mathrm{~S}, 7 R, 8 R, 8 \mathrm{a} S)-5 \mathrm{a}-(4-$ bromophenyl)-3-chloro-8a-hydroxy-8-methylsulfonyloxy-6-phenyl7,8-dihydro-6H-cyclopenta[4,5]furo[1,2-b]pyridine-7-carboxylate (18) $(775 \mathrm{mg}, 1.30 \mathrm{mmol})$ in DMSO $(13 \mathrm{~mL})$ was added potassium cyanide (189 mg, $2.90 \mathrm{mmol}$ ) and the mixture was stirred at $\mathrm{rt}$ under argon. After stirring for $16 \mathrm{~h}$ the mixture was diluted with EtOAc and washed brine $(2 \mathrm{x})$, then dried $\left(\mathrm{Na}_{2} \mathrm{SO}_{4}\right)$, filtered and concentrated. Purification by column chromatography $\left(\mathrm{SiO}_{2}, 0-45 \%\right.$ ethyl acetate/hexane) yielded $469 \mathrm{mg}$ (0.892 mmol, 68\%) of 21 as a yellowish foam; HRMS (ESI) 
m/z: $[\mathrm{M}+\mathrm{H}]^{+}$Calcd for $\mathrm{C}_{25} \mathrm{H}_{19} \mathrm{BrClN}_{2} \mathrm{O}_{4}{ }^{+}$525.0211; Found 525.0219; ${ }^{\mathbf{1}} \mathbf{H}$ NMR (400 MHz, d6. DMSO) $\delta / \mathrm{ppm}=8.36(\mathrm{~d}, J=1.9 \mathrm{~Hz}, 1 \mathrm{H}), 7.85(\mathrm{~d}, J=1.9 \mathrm{~Hz}, 1 \mathrm{H}), 7.37(\mathrm{~d}, J=8.9 \mathrm{~Hz}, 2 \mathrm{H}), 7.13$ $(\mathrm{d}, J=8.9 \mathrm{~Hz}, 2 \mathrm{H}), 7.10-7.06(\mathrm{~m}, 3 \mathrm{H}), 6.96-6.90(\mathrm{~m}, 2 \mathrm{H}), 6.83(\mathrm{~s}, 1 \mathrm{H}), 4.22(\mathrm{~d}, J=10.5 \mathrm{~Hz}, 1 \mathrm{H})$, $4.15(\mathrm{dd}, J=13.1,10.5 \mathrm{~Hz}, 1 \mathrm{H}), 3.75(\mathrm{~d}, J=13.1 \mathrm{~Hz}, 1 \mathrm{H}), 3.53$ (s, 3H). ${ }^{13} \mathrm{C}$ NMR (101 MHz, d6-DMSO) $\delta / \mathrm{ppm}=170.5,151.1,149.7,142.3,134.8,133.2,132.5,130.2,129.6,128.1,127.9$, 127.3, 121.1, 119.6, 117.4, 101.4, 83.9, 57.0, 52.7, 47.7, 41.2.

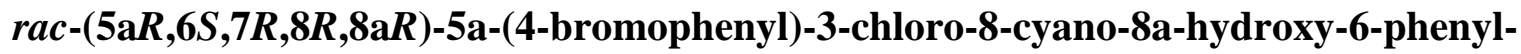
5a,7,8,8a-tetrahydro-6H-cyclopenta[4,5]furo[3,2-b]pyridine-7-carboxylic acid (22)

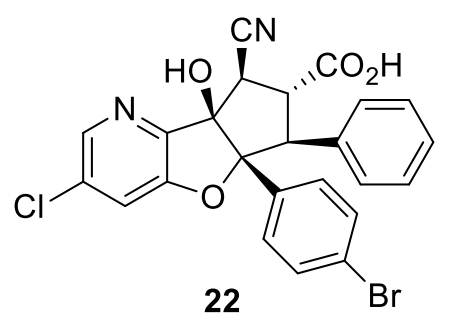

To rac-methyl (5aR,6S,7R,8R,8a $R)$-5a-(4-bromophenyl)-3-chloro-8cyano-8a-hydroxy-6-phenyl-7,8-dihydro-6H-cyclopenta-[4,5]furo[1,2-b]pyridine-7-carboxylate $\quad(\mathbf{2 1}) \quad(460 \quad \mathrm{mg}, \quad 0.875 \quad \mathrm{mmol})$ in methanol $(4.5 \mathrm{~mL})$ and $\mathrm{THF}(4.5 \mathrm{~mL})$ at $\mathrm{rt}$ was added $\mathrm{LiOH}(\mathrm{aq})$ $\left(2 \mathrm{M}, 6.3 \mathrm{~mL}, 12.6 \mathrm{mmol}\right.$ ) and the mixture was stirred at $40^{\circ} \mathrm{C}$ (water bath). After $1 \mathrm{~h}$ the mixture was cooled down to rt, and stirred for another $15 \mathrm{~min}$, then acidified with $2 \mathrm{M} \mathrm{HCl}(\mathrm{aq})(7 \mathrm{~mL})$ and diluted with EtOAc and water. The aq. phase was extracted thrice, and the combined organic phases were dried $\left(\mathrm{Na}_{2} \mathrm{SO}_{4}\right)$, filtered and concentrated to give $417 \mathrm{mg}(0.815 \mathrm{mmol}, 93 \%)$ of $\mathbf{2 2}$. The crude product was directly used in the next step without further purification. An analytically pure sample could be obtained by purification by reverse phase HPLC (C18, MeCN/water+0.1\%TFA). HRMS (ESI) m/z: [M+H] ${ }^{+}$ Calcd for $\mathrm{C}_{24} \mathrm{H}_{17} \mathrm{BrClN}_{2} \mathrm{O}_{4}{ }^{+}$511.0055; Found 511.0051; ${ }^{1} \mathrm{H}$ NMR (400 MHz, d $\mathbf{6}$-DMSO) $\delta / \mathrm{ppm}$ $=13.11(\mathrm{~b}, 1 \mathrm{H}), 8.36(\mathrm{~d}, J=2.0 \mathrm{~Hz}, 1 \mathrm{H}), 7.87(\mathrm{~d}, J=2.0 \mathrm{~Hz}, 1 \mathrm{H}), 7.40(\mathrm{~d}, J=8.8 \mathrm{~Hz}, 2 \mathrm{H}), 7.13$ $(\mathrm{d}, J=8.8 \mathrm{~Hz}, 2 \mathrm{H}), 7.11-7.08(\mathrm{~m}, 3 \mathrm{H}), 6.97-6.90(\mathrm{~m}, 2 \mathrm{H}), 6.81(\mathrm{~s}, 1 \mathrm{H}), 4.11(\mathrm{~d}, J=10.8 \mathrm{~Hz}, 1 \mathrm{H})$, $3.98(\mathrm{dd}, J=13.4,10.8 \mathrm{~Hz}, 1 \mathrm{H}), 3.70(\mathrm{~d}, J=13.4 \mathrm{~Hz}, 1 \mathrm{H}) ;{ }^{13} \mathbf{C}$ NMR (101 MHz, d6-DMSO) $\delta$ / $\mathrm{ppm}=171.5,151.0,149.7,142.1,134.7,133.2,132.3,130.1,129.5,128.1,127.7,127.2,121.0$, $119.5,117.3,101.5,83.8,57.2,48.2,41.4$. 
rac-(5a $R, 6 S, 8 R, 8 \mathrm{a} R)$-5a-(4-bromophenyl)-3-chloro-8a-hydroxy-6-phenyl-5a,7,8,8atetrahydro-6H-cyclopenta[4,5]furo[3,2-b]pyridine-8-carbonitrile (23)

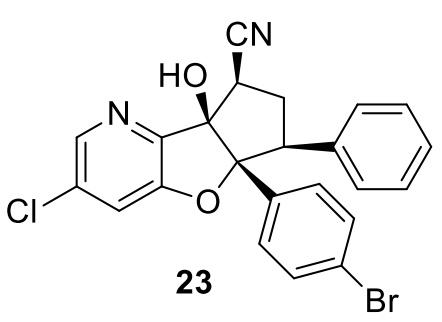

In a screw-cap vial, crude $r a c-(5 \mathrm{a} R, 6 S, 7 R, 8 R, 8 \mathrm{a} R)-5 \mathrm{a}-(4-$ bromophenyl)-3-chloro-8-cyano-8a-hydroxy-6-phenyl-7,8-dihydro6H-cyclopenta-[4,5]furo[1,2-b]pyridine-7-carboxylic acid (22) (387 $\mathrm{mg}, 0.756 \mathrm{mmol})$ and 1-hydroxypyridine-2-thione (144 mg, 1.13 mmol) were suspended in chloroform (7.5 mL). 2-methylpropane-2-

thiol $(2.1 \mathrm{~mL}, 0.168 \mathrm{~g}, 18.6 \mathrm{mmol})$ was added, the mixture was irradiated (light source: HDX XG1026 lamp, $250 \mathrm{~W}$ halogen bulb), and DCC (175 mg, $0.848 \mathrm{mmol})$ in $1.5 \mathrm{~mL} \mathrm{CHCl}_{3}$ was added dropwise under argon. The mixture was irradiated for $15 \mathrm{~min}$ with vigorous stirring without additional external heating. The mixture was then concentrated and dried in vacuo. Purification by repeated column chromatography (first column: $\mathrm{SiO}_{2}, 0-25 \%$ ethyl acetate/hexane; second column: $\mathrm{SiO}_{2}, 0-20 \%$ ethyl acetate/hexane) yielded $136 \mathrm{mg}(0.291 \mathrm{mmol}, 38 \%)$ of $\mathbf{2 3}$ as an offwhite foam; HRMS (ESI) m/z: [M+H] $]^{+}$Calcd for $\mathrm{C}_{23} \mathrm{H}_{17} \mathrm{BrClN}_{2} \mathrm{O}_{2}{ }^{+}$467.0156; Found 467.0144; ${ }^{1}$ H NMR (400 MHz, d $\mathbf{d}^{6}$-DMSO) $\delta / \mathrm{ppm}=8.32(\mathrm{~d}, J=2.0 \mathrm{~Hz}, 1 \mathrm{H}), 7.81(\mathrm{~d}, J=2.0 \mathrm{~Hz}, 1 \mathrm{H}), 7.36$ $(\mathrm{d}, J=8.7 \mathrm{~Hz}, 2 \mathrm{H}), 7.15-7.05(\mathrm{~m}, 5 \mathrm{H}), 6.98-6.93(\mathrm{~m}, 2 \mathrm{H}), 6.55(1 \mathrm{H}), 3.98(\mathrm{dd}, J=11.2,7.5 \mathrm{~Hz}$, 1H), $3.62(\mathrm{dd}, J=14.0,5.9 \mathrm{~Hz}, 1 \mathrm{H}), 2.80-2.69(\mathrm{~m}, 1 \mathrm{H}), 2.58-2.52(\mathrm{~m}, 1 \mathrm{H}) ;{ }^{13} \mathbf{C} \mathbf{N M R}(\mathbf{1 0 1} \mathbf{M H z}$, d6-DMSO) $\delta / \mathrm{ppm}=151.3,150.3,142.0,136.8,134.0,132.3,130.1,129.7,128.0,128.0,127.1$, $120.9,119.2,119.1,102.0,85.5,54.0,37.3,32.2$.

rac-(5aR,6S,8R,8aR)-8-(aminomethyl)-5a-(4-bromophenyl)-3-chloro-6-phenyl-5a,6,7,8tetrahydro-8aH-cyclopenta[4,5]furo[3,2-b]pyridin-8a-ol (TFA salt) (24)

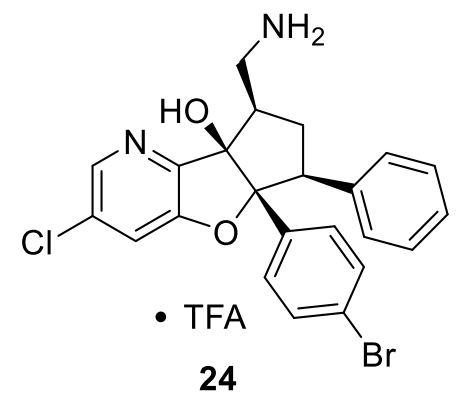
To a solution of $r a c-(5 \mathrm{a} R, 6 S, 8 R, 8 \mathrm{a} R)-5 \mathrm{a}$-(4-bromophenyl)-3-chloro8a-hydroxy-6-phenyl-7,8-dihydro-6H-cyclopenta[4,5]furo-[1,2b]pyridine-8-carbonitrile (23) $(134 \mathrm{mg}, 0.286 \mathrm{mmol})$ in THF $(2.9 \mathrm{~mL})$ at $0{ }^{\circ} \mathrm{C}$ was added lithium aluminum hydride $(27.2 \mathrm{mg}, 0.716 \mathrm{mmol})$ and the mixture was stirred at rt. After $30 \mathrm{~min} 0.1 \mathrm{~mL}$ water were added at $0{ }^{\circ} \mathrm{C}$, followed by $0.1 \mathrm{~mL} 12.5 \% \mathrm{NaOH}$ (aq) and ca. $200 \mathrm{mg}$ of $\mathrm{Na}_{2} \mathrm{SO}_{4}$. The mixture was stirred for $10 \mathrm{~min}$ at $\mathrm{rt}$, then filtered through celite (rinsed with DCM) and concentrated. The crude product was taken up in $2 \mathrm{~mL} \mathrm{DCM}$ and treated with trifluoroacetic acid $(0.03 \mathrm{~mL}, 0.39000 \mathrm{mmol})$, then concentrated, to give $176 \mathrm{mg}$ of the crude product. $80 \mathrm{mg}$ of 
this crude product were purified by preparative HPLC (C18, MeCN/water+0.1\% TFA) for analytical purposes, providing $22 \mathrm{mg}$ (0.037 mmol, 28\%) of 24. HRMS (ESI) m/z: $[\mathrm{M}+\mathrm{H}]^{+} \mathrm{Calcd}$ for $\mathrm{C}_{23} \mathrm{H}_{21} \mathrm{BrClN}_{2} \mathrm{O}_{2}{ }^{+}$471.0469; Found 471.0474; ${ }^{1} \mathrm{H}$ NMR (400 MHz, d $\mathbf{6}$-DMSO, TFA salt) $\delta$ / ppm $=8.29(\mathrm{~d}, J=2.0 \mathrm{~Hz}, 1 \mathrm{H}), 7.84(\mathrm{~d}, J=2.0 \mathrm{~Hz}, 1 \mathrm{H}), 7.84-7.73(\mathrm{~b}, 3 \mathrm{H}), 7.33(\mathrm{~d}, J=8.7 \mathrm{~Hz}$, 2H), 7.15-7.04 (m, 5H), 6.99-6.95 (m, 2H), 6.15 (s, 1H), 3.70-3.62 (m, 1H), 3.45-3.35 (m, 1H), 3.24-3.13 (m, 1H), 2.87-2.75 (m,1 H), 2.40-2.23 (m, 2H); ${ }^{13}$ C NMR (101 MHz, d ${ }^{6}$-DMSO, TFA salt) $\delta / \mathrm{ppm}=151.9,151.1,141.3,137.7,134.7,131.6,129.7,129.6,127.8,127.7,126.6,120.4$, $118.8,103.2,86.0,54.6,44.1,32.0$.

\section{4-((5aR,6S,8R,8aR)-8-(aminomethyl)-3-chloro-8a-hydroxy-6-phenyl-6,7,8,8a-tetrahydro-}

\section{5aH-cyclopenta[4,5]furo[3,2-b]pyridin-5a-yl)benzonitrile (TFA salt) (25)}

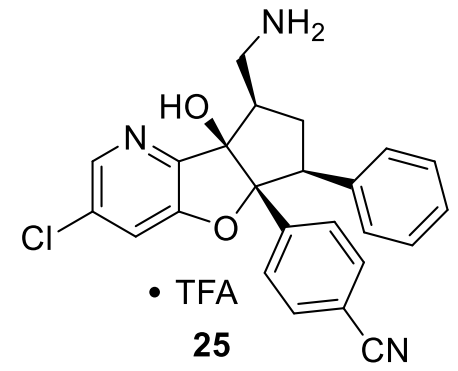

In a $2 \mathrm{~mL}$ microwave vial $\mathrm{rac}-(5 \mathrm{a} R, 6 S, 8 R, 8 \mathrm{a} R)-8$-(aminomethyl)-5a-

(4-bromophenyl)-3-chloro-6-phenyl-5a,6,7,8-tetrahydro-8aHcyclopenta[4,5]furo[3,2-b]pyridin-8a-ol (TFA salt) (24) (48 mg, $0.082 \mathrm{mmol})$ was dissolved in DMF $(0.7 \mathrm{~mL})$ and water $(0.07 \mathrm{~mL})$. Zinc cyanide (39 $\mathrm{mg}, 0.33 \mathrm{mmol}$ ) and zinc (3 $\mathrm{mg}, 0.05 \mathrm{mmol})$ were added, and the mixture was degassed by bubbling argon through it for 5 min. Dppf (14.0 mg, $0.0253 \mathrm{mmol})$ and $\mathrm{Pd}_{2}(\mathrm{dba})_{3}(10.8 \mathrm{mg}, 0.0118 \mathrm{mmol})$ were added and the mixture was degassed for another $5 \mathrm{~min}$, then incubated at $100^{\circ} \mathrm{C}$ for $2.25 \mathrm{~h}$ in total (Biotage Initiator focused beam microwave reactor). The mixture was then filtered, diluted with $\mathrm{MeCN}$, DMSO and water and subjected to purification by preparative HPLC (C18, $\mathrm{MeCN} /$ water+0.1\% TFA) to give compound 25. Yield: $13.8 \mathrm{mg}(0.0259 \mathrm{mmol}, 33 \%$ over two steps), white solid. HRMS (ESI) m/z: $[\mathrm{M}+\mathrm{H}]^{+}$Calcd for $\mathrm{C}_{24} \mathrm{H}_{21} \mathrm{ClN}_{3} \mathrm{O}_{2}{ }^{+}$418.1317; Found

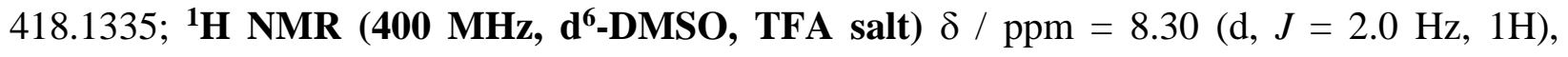
7.97-7.87 (b, 3H), 7.86 (d, $J=2.0 \mathrm{~Hz}, 1 \mathrm{H}), 7.60$ (d, $J=8.4 \mathrm{~Hz}, 2 \mathrm{H}), 7.37$ (d, $J=8.4 \mathrm{~Hz}, 2 \mathrm{H})$, 7.16-7.02 (m, 3H), 7.02-6.90 (m, 2H), $6.22(\mathrm{bs}, 1 \mathrm{H}), 3.79-3.70(\mathrm{~m}$, partially obscured by water peak, $1 \mathrm{H}$ expected), 3.47-3.35 (m, 1H), 3.27-3.13 (m, 1H), 2.89-2.77 (m, 1H), 2.48-2.36 (m, 1H), 2.36-2.24 (m, 1H); ${ }^{13}$ C NMR (126 MHz, d6-DMSO, TFA salt) $\delta / \mathrm{ppm}=151.7,151.1$, $141.7,141.2$, 137.5, 131.9, 130.9, 128.5, 128.0, 127.8, 126.9, 119.1, 118.9, 109.8, 103.4, 86.5, $55.2,44.6,32.1$. 


\section{Mechanistic investigations of stereoretentive and stereoinvertive displacements}

\subsection{Mechanistic investigation of the stereoretentive displacement $\mathbf{1 4} \rightarrow \mathbf{1 6}$}

3.1.1 Synthesis and characterization of epoxide $\mathbf{1 7}$

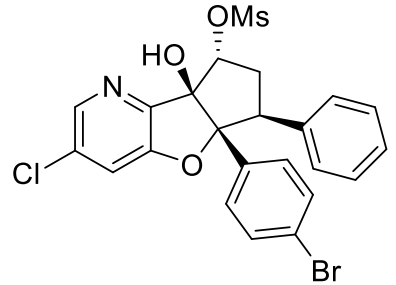

14

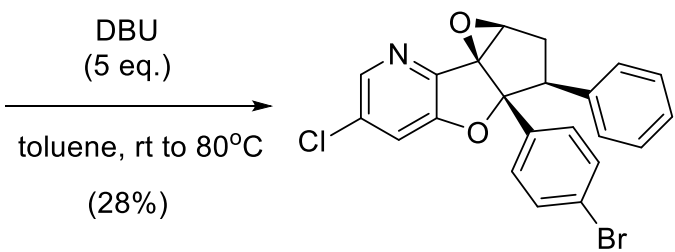

17

rac-(1aS,3S,3aR,8bS)-3a-(4-bromophenyl)-6-chloro-3-phenyl-1a,2,3,3atetrahydrooxireno[2", $\left.3^{\prime \prime}: 1^{\prime}, 5^{\prime}\right]$ cyclopenta[1',2':4,5]furo[3,2-b]pyridine (17)<smiles>Clc1cnc2c(c1)O[C@]1(c3ccc(Br)cc3)[C@H](c3ccccc3)C[C@@H]3O[C@]231</smiles>

17

In a flame dried vial, [(5aR,6S,8R,8aS)-5a-(4-bromophenyl)-3chloro-8a-hydroxy-6-phenyl-7,8-dihydro-6H-cyclopenta[4,5]furo[1,2-b]pyridin-8-yl] methanesulfonate (14) $(17.7 \mathrm{mg}, 0.0330 \mathrm{mmol})$ was dissolved in toluene $(0.5 \mathrm{~mL})$. DBU ( $25 \mathrm{uL}, 26 \mathrm{mg}, 0.17 \mathrm{mmol})$ was added, and the mixture was stirred at $50{ }^{\circ} \mathrm{C}$ in a heating block. After 20 min ca. $50 \%$ conversion was observed by TLC. After another 25 min the mixture warmed up to $65^{\circ} \mathrm{C}$. After another $45 \mathrm{~min}$ the mixture wss warmed up to $80{ }^{\circ} \mathrm{C}$. After another $45 \mathrm{~min}$ another $25 \mu \mathrm{L}$ DBU were added. Stirring at $80{ }^{\circ} \mathrm{C}$ was continued for $15 \mathrm{~min}$, after which the mixture was cooled down to rt. The reaction was quenched with $\mathrm{NH}_{4} \mathrm{Cl}(\mathrm{aq})$ and extracted $(3 \mathrm{x}$ DCM). The combined organic phases were dried $\left(\mathrm{Na}_{2} \mathrm{SO}_{4}\right)$, filtered and concentrated. Purification by column chromatography $\left(\mathrm{SiO}_{2}, 0-30 \%\right.$ ethyl acetate/hexane) followed by preparative TLC $\left(\mathrm{SiO}_{2}, \mathrm{EtOAc} / \mathrm{Hex}=1 / 1\right)$ gave $4.0 \mathrm{mg}(0.0091 \mathrm{mmol}, 28 \%$ yield $)$ of epoxide 17 as a white solid. HRMS (ESI) m/z: $[\mathrm{M}+\mathrm{H}]^{+}$Calcd for $\mathrm{C}_{22} \mathrm{H}_{16} \mathrm{BrClNO}_{2}{ }^{+} 440.0047[\mathrm{M}+\mathrm{H}]^{+}$; Found 440.0034; ${ }^{1} \mathbf{H}$ NMR (400 MHz, CDCl $) \delta /$ ppm $=8.17(\mathrm{~d}, J=1.9 \mathrm{~Hz}, 1 \mathrm{H}), 7.29(\mathrm{~d}, J=1.9 \mathrm{~Hz}, 1 \mathrm{H}), 7.23-7.03$ (m, 9H), 4.60 (dd, $J=12.2,9.3 \mathrm{~Hz}, 1 \mathrm{H}), 4.27$ (d, $J=3.8 \mathrm{~Hz}, 1 \mathrm{H}), 2.72$ (dd, $J=14.8,9.3,3.8 \mathrm{~Hz}$, 1H), 2.57 (dd, $J=14.8,12.2 \mathrm{~Hz}, 1 \mathrm{H}) ;{ }^{13} \mathbf{C}$ NMR (101 MHz, CDCl$) \delta / \mathrm{ppm}=155.7,142.6,141.7$, $136.9,135.3,133.1,130.8,129.0,128.4,128.2,127.2,122.1,118.1,95.3,78.7,67.2,63.6,32.7$. 


\subsubsection{LCMS studies}

The conversion of $\mathbf{1 4}$ to $\mathbf{1 5}$ was studied by LCMS. Samples of the reaction mixture were taken at different time points from $\mathrm{t}=0 \mathrm{~min}$ to $\mathrm{t}=1.5 \mathrm{~h}$ and analyzed. LCMS chromatograms of compounds 14, 17, and 15 were obtained and used as references. The results are in agreement with the reaction pathway shown below. 
Proposed reaction pathway:

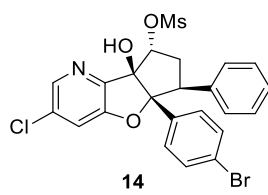

$t_{R}($ LCMS $)=2.70 \mathrm{~min}$

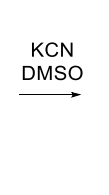

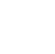

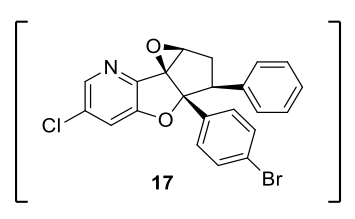

$t_{R}($ LCMS $)=3.04 \mathrm{~min}$

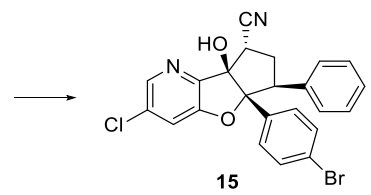

$t_{R}($ LCMS $)=2.65 \mathrm{~min}$

\section{LCMS of reaction mixture at different timepoints:}

$\mathrm{t}=2 \min$

- Vetector A, Channel 1 trom Sample 391 (CN-134-088-2mın-rt) of 134.witt

Max. 4.7e5

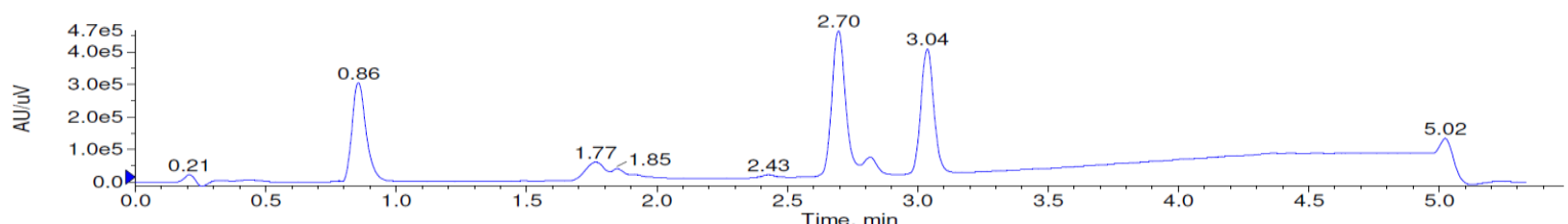
$\mathrm{t}=10 \mathrm{~min}$

- Vetector A, Lnannel 1 trom sample 392 (CN-134-U88-1 Umın-rt) or 134.WITt

Max. b.bes

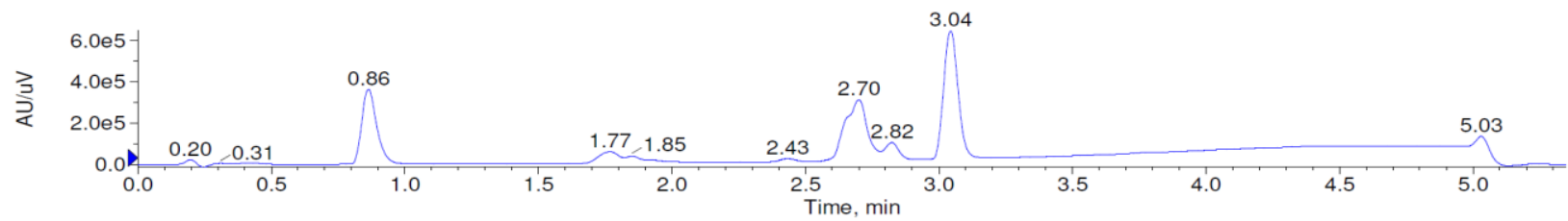

$\mathrm{t}=30 \mathrm{~min}$

- Vetector A, Channel 1 trom Sample 393 (CN-134-U88-3Umın-rt) of 134.witt

Max. 6.0e5

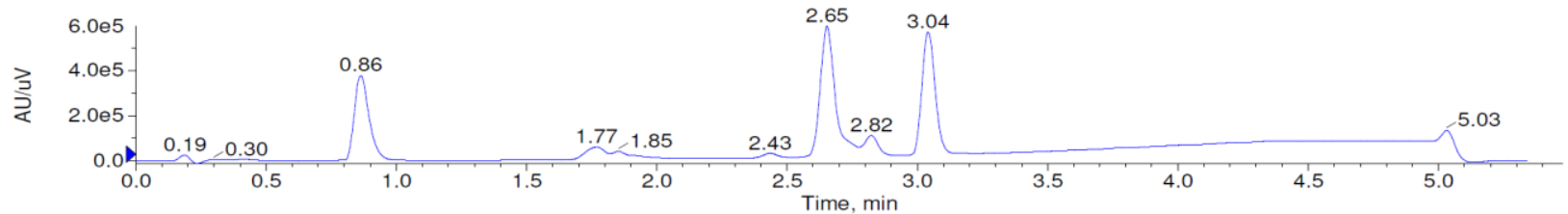

$\mathrm{t}=1 \mathrm{~h}$

- vetector A, Lnannel 1 trom sample 3ys (LiN-134-U88-1n-rt) or 134.Wiाt

Max. /.2es

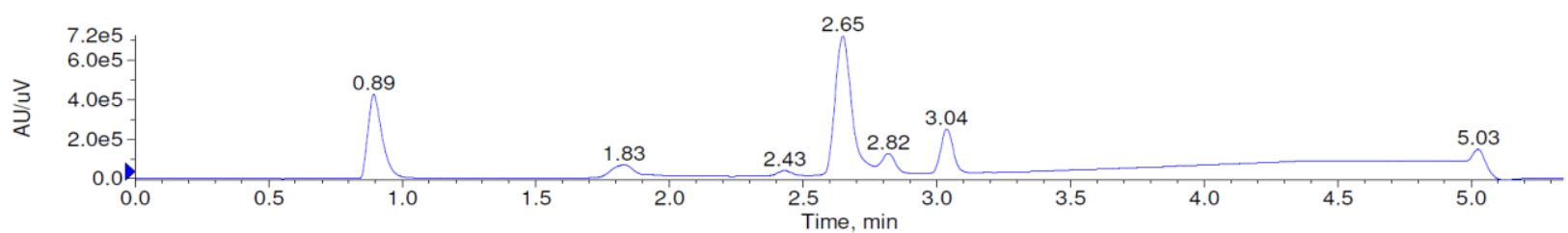

$\mathrm{t}=1.5 \mathrm{~h}$

- Detector A, Channel 1 from Sample 398 (CN-134-088-1.5h-rt) of 134.witt

Max. 5.6e5

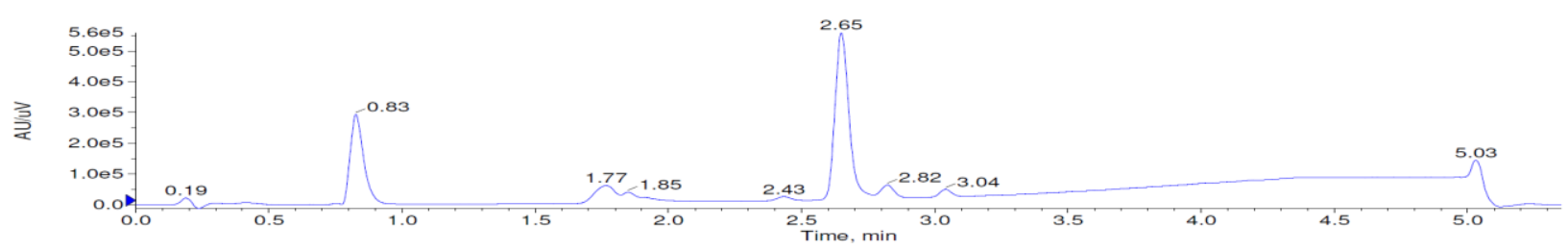


LCMS of 14 (starting material):<smiles>CO[C@H]1C[C@H](c2ccccc2)[C@@]2(c3ccc(Br)cc3)Oc3cc(Cl)cnc3[C@]12O</smiles>

Chemical Formula: $\mathrm{C}_{23} \mathrm{H}_{19} \mathrm{BrClNO}_{5} \mathrm{~S}$

Exact Mass: 534.9856

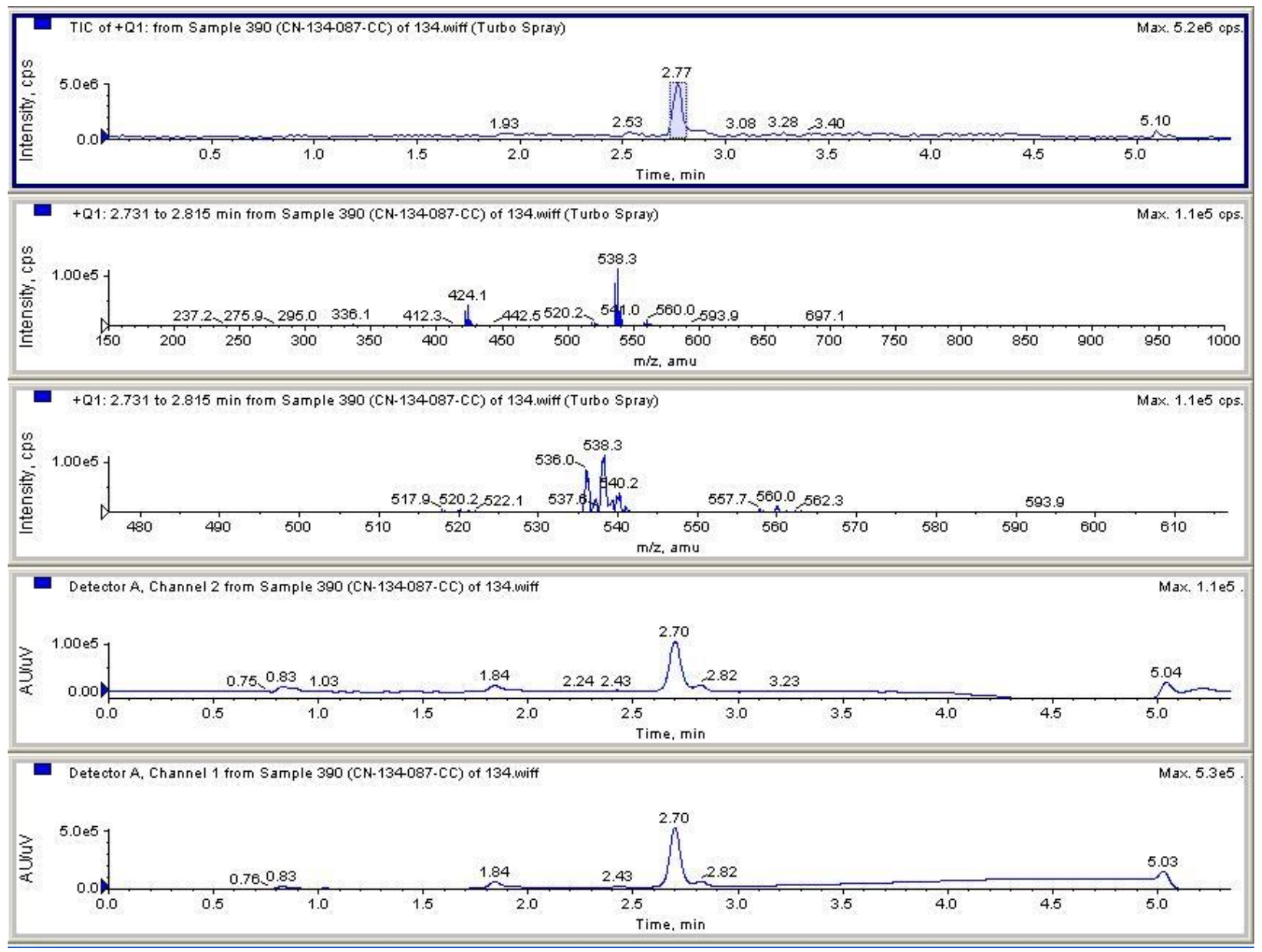


LCMS of 17 (separately synthesized and characterized by LCMS and NMR):<smiles>Clc1cnc2c(c1)O[C@]1(c3ccc(Br)cc3)[C@H](c3ccccc3)C[C@@H]3O[C@]231</smiles>

Chemical Formula: $\mathrm{C}_{22} \mathrm{H}_{15} \mathrm{BrCINO}_{2}$ Exact Mass: 438.9975

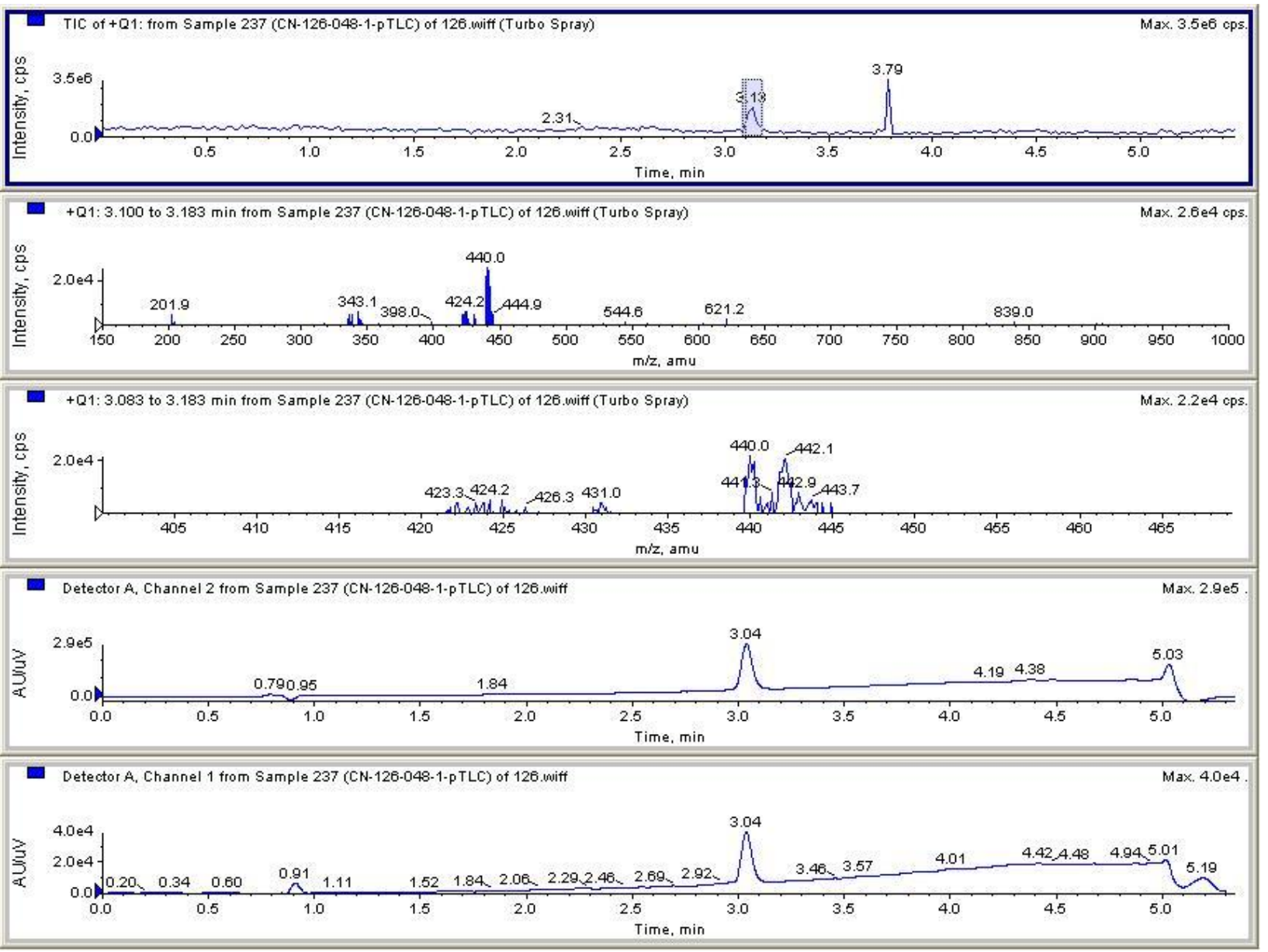




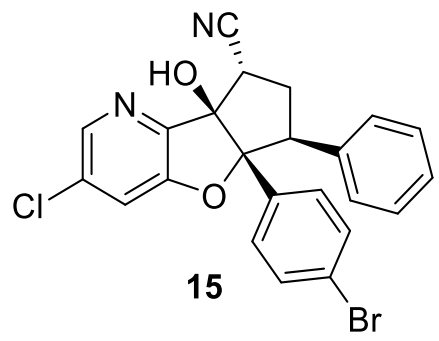

Chemical Formula: $\mathrm{C}_{23} \mathrm{H}_{16} \mathrm{BrClN}_{2} \mathrm{O}_{2}$

Exact Mass: 466.0084

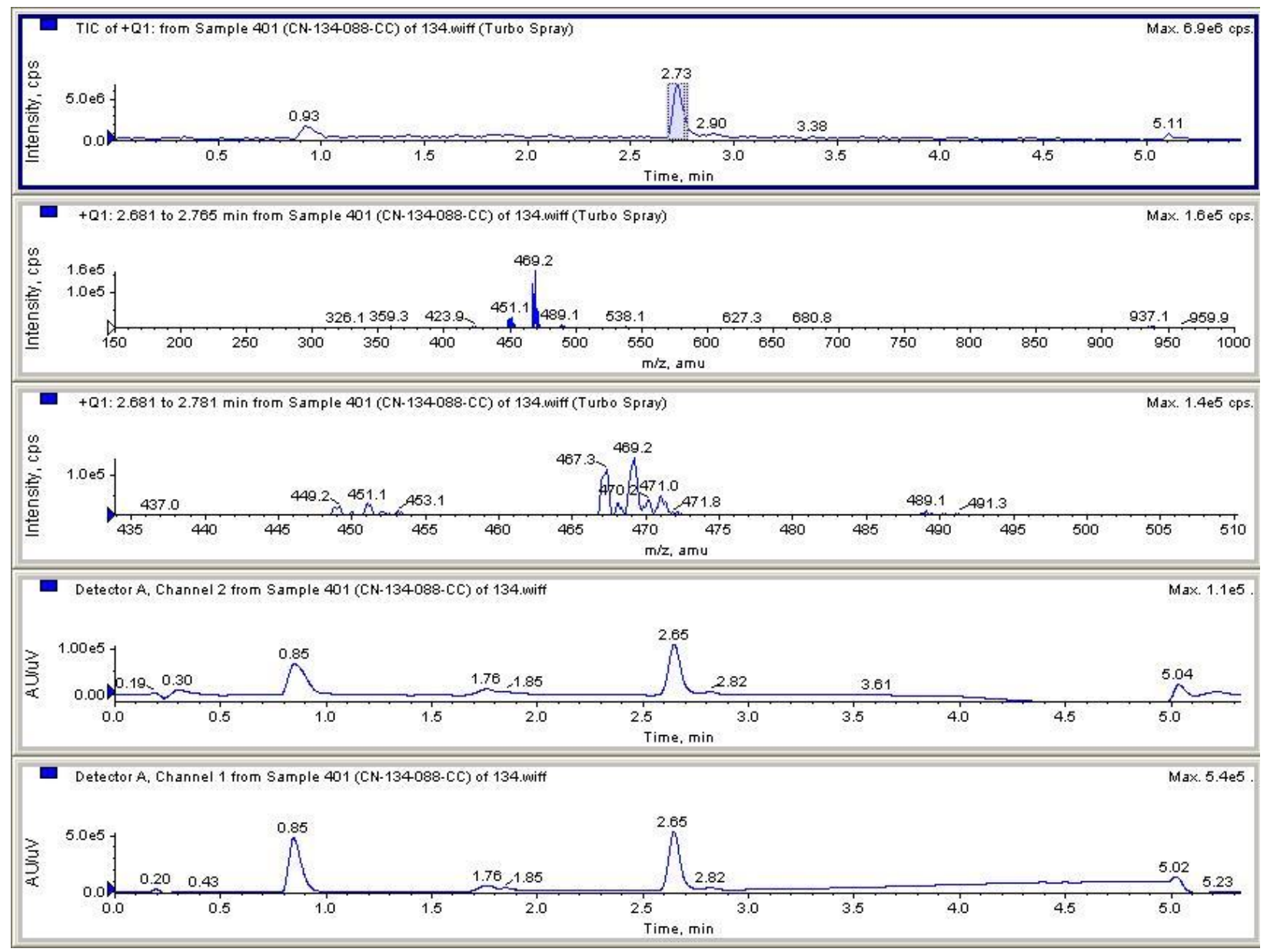


3.2 Mechanistic investigation of the stereoinvertive displacement $\mathbf{1 8} \rightarrow \mathbf{2 1}$

3.2.1 Synthesis and characterization of epoxide $\mathbf{1 9}$

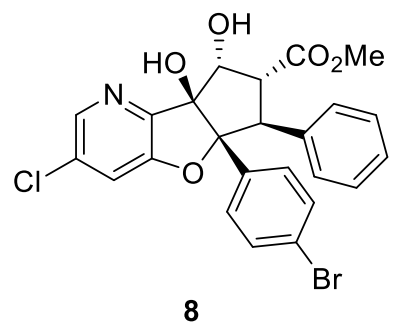

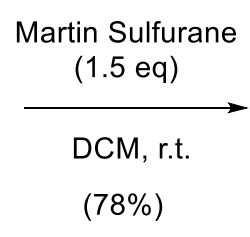

$(78 \%)$

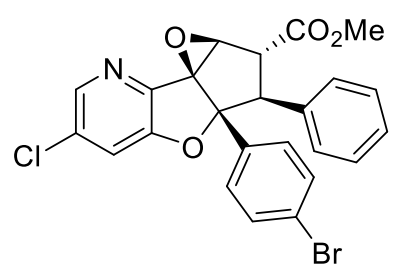

19

rac-methyl $\quad(1 \mathrm{a} S, 2 R, 3 S, 3 \mathrm{a} R, 8 \mathrm{~b} S)$-3a-(4-bromophenyl)-6-chloro-3-phenyl-1a,2,3,3a-tetrahydro-oxireno[2",3"':1',5']cyclopenta[1',2':4,5]furo[3,2-b]pyridine-2-carboxylate (19)<smiles></smiles>

19

rac-Methyl $(5 \mathrm{a} R, 6 S, 7 R, 8 R, 8 \mathrm{a} S)$-5a-(4-bromophenyl)-3-chloro-8,8adihydroxy-6-phenyl-7,8-dihydro-6H-cyclopenta[4,5]furo[1,2-

b]pyridine-7-carboxylate (8) $(42 \mathrm{mg}, 0.081 \mathrm{mmol})$ was dissolved in DCM $(0.6 \mathrm{~mL})$ under argon. Martin sulfurane $(82 \mathrm{mg}, 0.12 \mathrm{mmol})$ was added, and the mixture was stirred at rt. The mixture turned dark red. After $6 \mathrm{~h}$ the reaction was quenched with $\mathrm{NH}_{4} \mathrm{Cl}(\mathrm{aq})$. The mixture was extracted with DCM (3x), and the combined organic phases were dried $\left(\mathrm{Na}_{2} \mathrm{SO}_{4}\right)$, filtered and concentrated. Purification by repeated column chromatography $\left(\mathrm{SiO}_{2}, 0-30 \%\right.$ ethyl acetate/hexane, then $0-20 \%$ ethyl acetate/hexane) yielded $29.6 \mathrm{mg}(0.0594 \mathrm{mmol}, 73 \%)$ of 19 as a light orange solid; HRMS (ESI) m/z: $[\mathrm{M}+\mathrm{H}]^{+}$Calcd for $\mathrm{C}_{24} \mathrm{H}_{18} \mathrm{BrClNO}_{4}{ }^{+}$498.0102; Found 498.0082; ${ }^{1} \mathbf{H}$ NMR (400 MHz, $\left.\mathbf{C D C l}_{3}\right) \delta / \mathrm{ppm}=8.17(\mathrm{~d}, J=1.9 \mathrm{~Hz}, 1 \mathrm{H}), 7.29(\mathrm{~d}, J=1.9 \mathrm{~Hz}, 1 \mathrm{H}), 7.23-7.19(\mathrm{~m}, 2 \mathrm{H}), 7.15-7.10$ (m, 5H), 7.05-7.00 (m, 2H), $4.91(\mathrm{~d}, J=11.8 \mathrm{~Hz}, 1 \mathrm{H}), 4.37$ (d, $J=0.6 \mathrm{~Hz}, 1 \mathrm{H}), 3.72(\mathrm{~s}, 3 \mathrm{H}), 3.72$

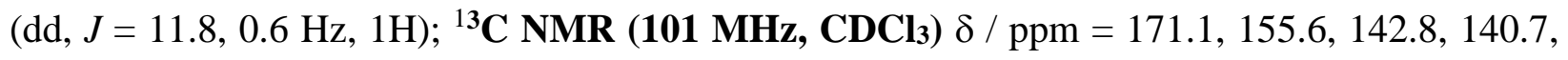
135.2, 135.0, 133.5, 130.9, 128.9, 128.5, 128.2, 127.7, 122.4, 118.3, 94.7, 78.1, 68.4, 67.4, 53.0, 50.0 . 
3.2.2 Synthesis and characterization of enoate $\mathbf{2 0}$

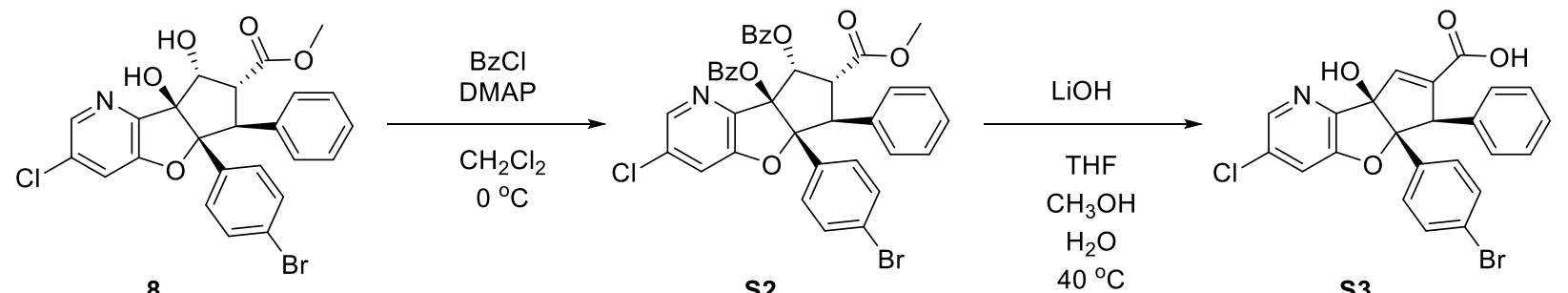

S2

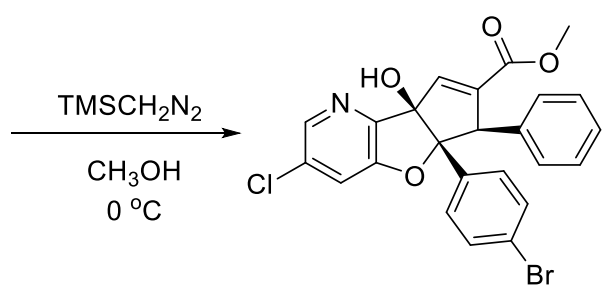

20

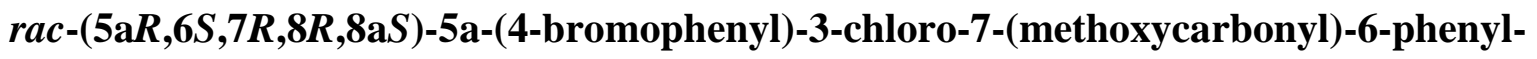
5a,6,7,8-tetrahydro-8aH-cyclopenta[4,5]furo[3,2-b]pyridine-8,8a-diyl dibenzoate (S2)

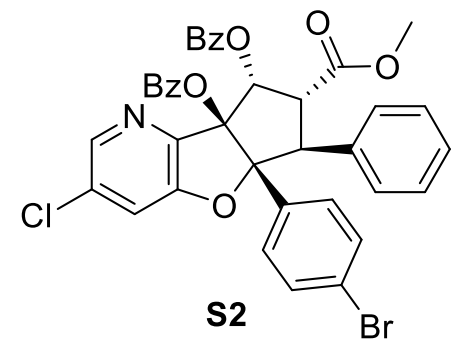

rac-methyl $(5 \mathrm{a} R, 6 S, 7 R, 8 R, 8 \mathrm{a} S)-5 \mathrm{a}$-(4-bromophenyl)-3-chloro-8,8adihydroxy-6-phenyl-5a,7,8,8a-tetrahydro-6H-cyclopenta[4,5]furo[3,2-b]pyridine-7-carboxylate (8) (150.0 $\mathrm{mg}, 0.2903 \mathrm{mmol})$ was dissolved in dichloromethane $(1.5 \mathrm{~mL})$ and the solution was cooled to

$0{ }^{\circ} \mathrm{C}$. Triethylamine $(0.45 \mathrm{~mL}, 0.33 \mathrm{~g}, 3.2 \mathrm{mmol})$, benzoyl chloride (0.15 mL, $0.18 \mathrm{~g}, 1.3 \mathrm{mmol})$, and 4-dimethylaminopyridine (19.5 mg, $0.160 \mathrm{mmol})$ were added sequentially. After $20 \mathrm{~min}$ the reaction mixture was warmed to room temperature. After $2 \mathrm{~h}$ the reaction mixture was poured mixture onto saturated aqueous sodium bicarbonate and diluted with ethyl acetate. The aqueous layer was extracted with ethyl acetate three times. The combined organic material was washed with brine, dried over magnesium sulfate and concentrated. Purification via silica gel chromatography (0-100 \% ethyl acetate in dichloromethane) afforded the desired product S2 as a tan resin. Yield: $173 \mathrm{mg}(238 \mathrm{mmol}, 82 \%)$;RMS (ESI) m/z: [M+H] ${ }^{+}$ Calcd for $\mathrm{C}_{38} \mathrm{H}_{28} \mathrm{BrClNO}_{7}{ }^{+}$724.0732; Found $\mathrm{m} / z$ 724.0695; ${ }^{1} \mathbf{H}$ NMR (400 MHz, CDCl + TMS) $\delta / \mathrm{ppm}=7.90(\mathrm{~d}, J=2.0 \mathrm{~Hz}, 1 \mathrm{H}), 7.86-7.82(\mathrm{~m}, 2 \mathrm{H}), 7.82-7.77(\mathrm{~m}, 2 \mathrm{H}), 7.59-7.53(\mathrm{~m}, 1 \mathrm{H})$, 7.53-7.48 (m, 1H), $7.47(\mathrm{~d}, J=2.0 \mathrm{~Hz}, 1 \mathrm{H}), 7.43-7.37(\mathrm{~m}, 2 \mathrm{H}), 7.37-7.31(\mathrm{~m}, 2 \mathrm{H}), 7.19-7.06(\mathrm{~m}$, $7 \mathrm{H}), 6.91(\mathrm{~d}, J=7.4 \mathrm{~Hz}, 1 \mathrm{H}), 6.90-6.86(\mathrm{~m}, 2 \mathrm{H}), 4.39$ (d, J=14.7 Hz, 1H), 4.18 (dd, J = 14.7, 7.4 Hz, 1H), 3.50 (s, 3H); ${ }^{13}$ C NMR (101 MHz, CDCl3 + TMS) $\delta / \mathrm{ppm}=168.4,165.2,163.7,154.0$, 
$143.8,142.5,134.7,133.6,133.6,133.6,133.5,130.9,129.9,129.8,129.2,129.1,128.5,128.5$, $128.3,127.8,127.7,127.4,122.1,118.6,100.5,94.1,78.4,57.2,52.6,48.6$.

rac-(5a $R, 6 R, 8 \mathrm{a} R)$-5a-(4-bromophenyl)-3-chloro-8a-hydroxy-6-phenyl-5a,8a-dihydro-6Hcyclopenta[4,5]furo[3,2-b]pyridine-7-carboxylic acid (S3)<smiles>O=C(O)C1=C[C@]2(O)c3ncc(Cl)cc3O[C@@]2(c2ccc(Br)cc2)[C@H]1c1ccccc1</smiles>

S3 rac-(5a $R, 6 S, 7 R, 8 R, 8 \mathrm{a} S)$-5a-(4-bromophenyl)-3-chloro-7-(methoxycarbonyl)-6-phenyl-5a,6,7,8-tetrahydro-8aH-cyclopenta[4,5]furo[3,2-b]pyridine-8,8a-diyl dibenzoate (S2) (80.0 $\mathrm{mg}, 0.110 \mathrm{mmol}$ ) was dissolved in THF $(1.5 \mathrm{~mL})$, water $(1.5 \mathrm{~mL})$, and methanol $(1.5 \mathrm{~mL})$. Solid lithium hydroxide $(5.0 \mathrm{mg}, 0.22 \mathrm{mmol})$ was added at room temperature and the mixture subsequently stirred at $40^{\circ} \mathrm{C}$ in a heating block. After $18 \mathrm{~h}$ the reaction mixture was cooled to room temperature. The solvent was removed in vacuo and the residue taken up in a 1:1 mixture of methanol and DMSO with 1 drop of TFA. Purification via preparative HPLC (C18, MeCN/water+0.1\%TFA) afforded the desired product $\mathbf{S 3}$ as a white solid. Yield: $25 \mathrm{mg}(52 \mathrm{mmol}, 47 \%)$; HRMS (ESI) $\mathrm{m} / \mathrm{z}$ : $[\mathrm{M}+\mathrm{H}]^{+}$Calcd for $\mathrm{C}_{23} \mathrm{H}_{16} \mathrm{BrClNO}_{4}{ }^{+}$483.9946; Found 483.9919; ${ }^{1} \mathrm{H}$ NMR (400 MHz, CD 3 OD) $\delta / \mathrm{ppm}=8.12$ (bs, $1 \mathrm{H}), 7.52(\mathrm{~d}, J=1.8 \mathrm{~Hz}, 1 \mathrm{H}), 7.16-7.11(\mathrm{~m}, 5 \mathrm{H}), 7.09-7.05(\mathrm{~m}, 2 \mathrm{H}), 7.03(\mathrm{~d}, J=1.5 \mathrm{~Hz}, 1 \mathrm{H}), 6.98-$ $6.94(\mathrm{~m}, 2 \mathrm{H}), 4.72(\mathrm{~d}, J=1.5 \mathrm{~Hz}, 1 \mathrm{H}) ;{ }^{13} \mathbf{C}$ NMR (101 MHz, CD $\left.\mathbf{3 O D}\right) \delta / \mathrm{ppm}=167.0,154.5$, 150.1, 142.7, 142.4, 141.4, 138.7, 135.6, 133.9, 130.9, 130.7, 130.3, 129.1, 128.3, 122.5, 119.5, 104.5, 91.7, 63.7 .

rac-methyl (5aR,6R,8aR)-5a-(4-bromophenyl)-3-chloro-8a-hydroxy-6-phenyl-5a,8adihydro-6H-cyclopenta[4,5]furo[3,2-b]pyridine-7-carboxylate (20)

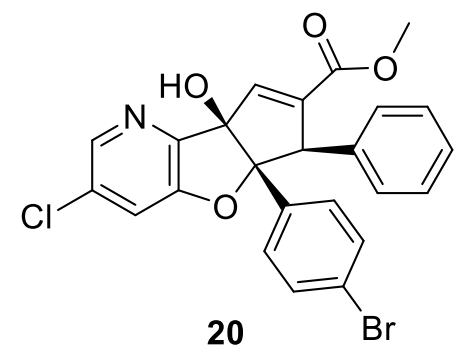

in ether) was added every $30 \mathrm{~min}(0.03 \mathrm{~mL}, 0.06 \mathrm{mmol}$ each time $)$ until the starting material was consumed as judged by LCMS. The solvent was removed in vacuo and purification via preparative 
HPLC (C18, MeCN/water+0.1\%TFA) afforded the desired product 20 as a white solid. Yield 5.7 mg (11 mmol, 69\%); HRMS (ESI) m/z: [M+H $]^{+}$Calcd for $\mathrm{C}_{24} \mathrm{H}_{18} \mathrm{BrClNO}_{4}{ }^{+}$498.0102; Found 498.0091; ${ }^{1} \mathbf{H}$ NMR (400 MHz, d $\mathbf{d}^{\mathbf{6}}$-DMSO) $\delta / \mathrm{ppm}=8.22(\mathrm{~d}, J=2.0 \mathrm{~Hz}, 1 \mathrm{H}), 7.71(\mathrm{~d}, J=2.0$ $\mathrm{Hz}, 1 \mathrm{H}), 7.21(\mathrm{~d}, J=8.8 \mathrm{~Hz}, 2 \mathrm{H}), 7.18-7.11(\mathrm{~m}, 3 \mathrm{H}), 7.05$ (d, $J=8.8 \mathrm{~Hz}, 2 \mathrm{H}), 6.98-6.92(\mathrm{~m}, 4 \mathrm{H})$, $4.67(\mathrm{~d}, J=1.6 \mathrm{~Hz}, 1 \mathrm{H}), 3.59$ (s, 3H); ${ }^{13} \mathbf{C}$ NMR (101 MHz, d ${ }^{6}$-DMSO) $\delta / \mathrm{ppm}=163.7,152.3$, $148.9,141.9,141.5,137.9,136.9,134.3,131.4,129.8,129.4,128.9,128.0,127.2,120.8,118.0$, $102.4,89.8,61.4,52.0$. 


\subsubsection{LCMS studies}

The conversion of $\mathbf{1 8}$ to $\mathbf{2 1}$ was studied by LCMS. Samples of the reaction mixture were taken at

different time points from $\mathrm{t}=0 \mathrm{~min}$ to $\mathrm{t}=17.5 \mathrm{~h}$ and analyzed. LCMS chromatograms of compounds 18, 19, 20, and 21 were obtained and used as references. The results are in agreement with the reaction pathway shown below. 


\section{Proposed reaction pathway:}

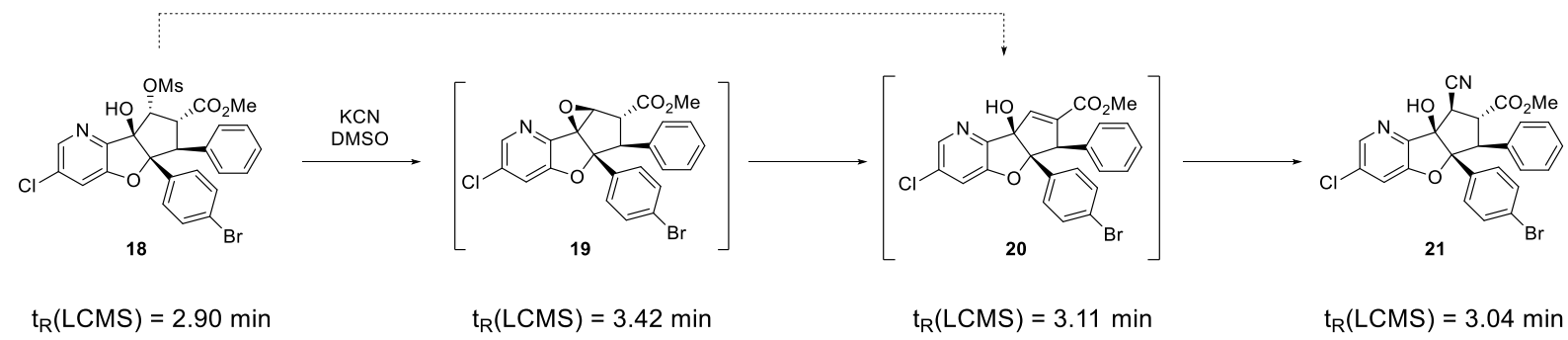

\section{LCMS of reaction mixture at different timepoints:}

$\mathrm{t}=0 \mathrm{~min}$ (before $\mathrm{KCN}$ addition):

- TWC of DAD Signal Data: from Sample 19 (CN-178-016-0min) of 178.wiff

Max. $2133.8 \mathrm{mAU}$

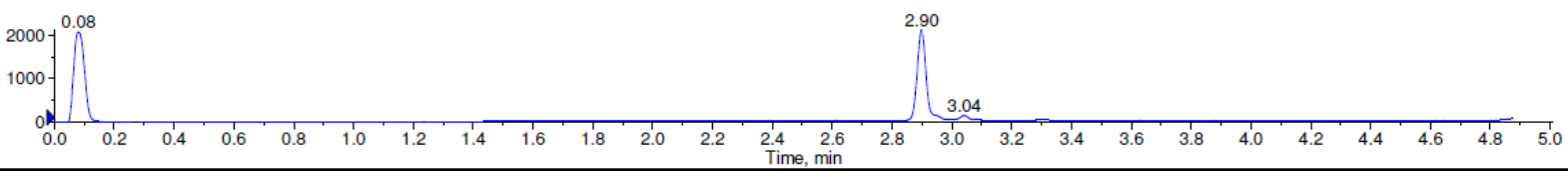

$\mathrm{t}=5 \mathrm{~min}:$

- TWC of DAD Signal Data: from Sample 16 (CN-178-016-5min) of 178.wiff

Max. 2017.1 mAU.

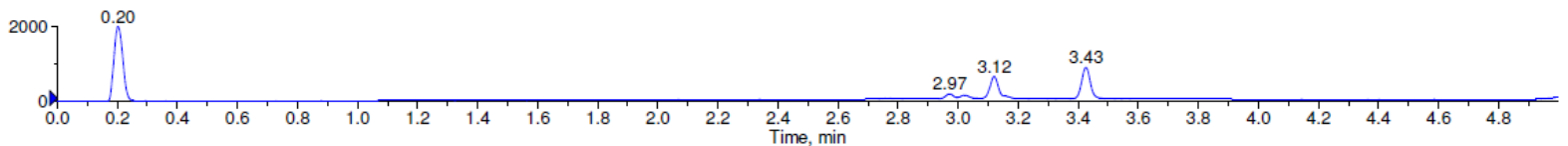

$\mathrm{t}=10 \mathrm{~min}:$

TWC of DAD Signal Data: from Sample 17 (CN-178-016-10min) of 178.wiff

Max. 2082.4 mAU.

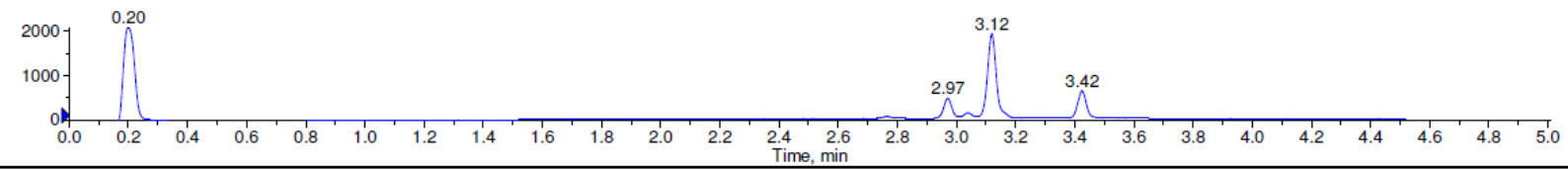

$\mathrm{t}=30 \mathrm{~min}:$

- TWC of DAD Signal Data: from Sample 18 (CN-178-016-30min) of 178.wiff

Max. 2093.9 mAU.

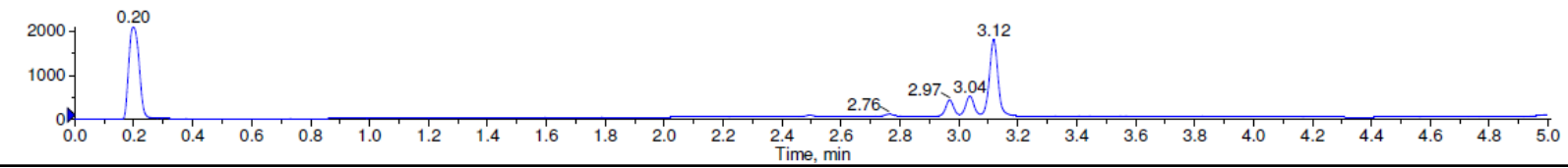

$\mathrm{t}=2 \mathrm{~h}$ :

TWC of DAD Signal Data: from Sample 23 (CN-178-016-2h-rt) of 178.wiff

Max. 2093.6 mAU

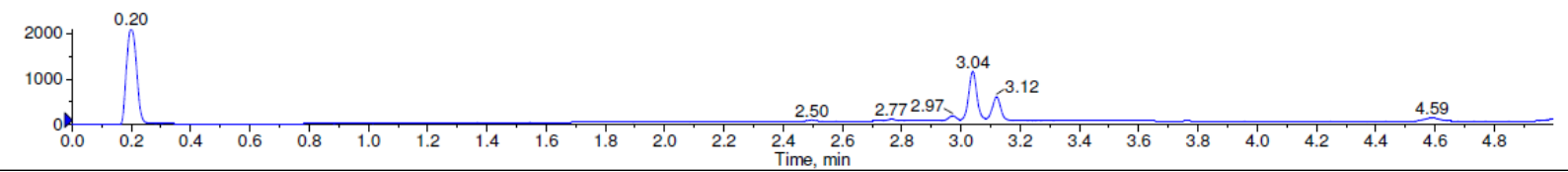

$\mathrm{t}=17.5 \mathrm{~h}:$

TWC of DAD Signal Data: from Sample 26 (CN-178-017.5h-rt) of 178.wiff

Max. 2130.2 mAU.

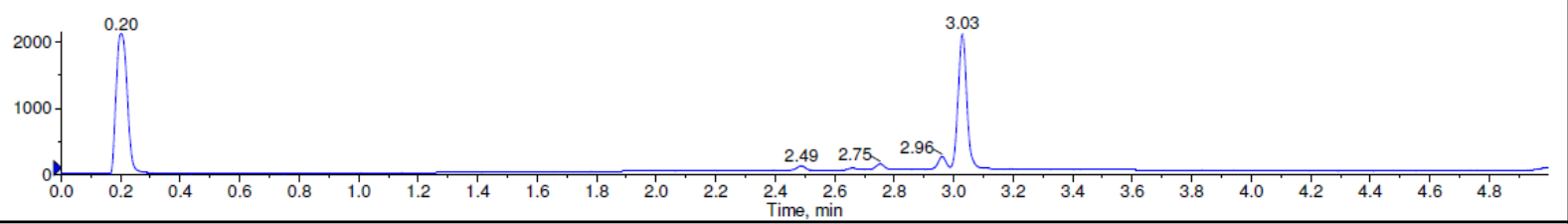




\section{LCMS of 18 (starting material) in DMSO before KCN addition:}<smiles>CO[C@H]1[C@H](c2ccccc2)[C@@]2(c3ccc(Br)cc3)Oc3cc(Cl)cnc3[C@]2(O)[C@@H]1OC</smiles>

Chemical Formula: $\mathrm{C}_{25} \mathrm{H}_{21} \mathrm{BrClNO}_{7} \mathrm{~S}$

Exact Mass: 592.9911

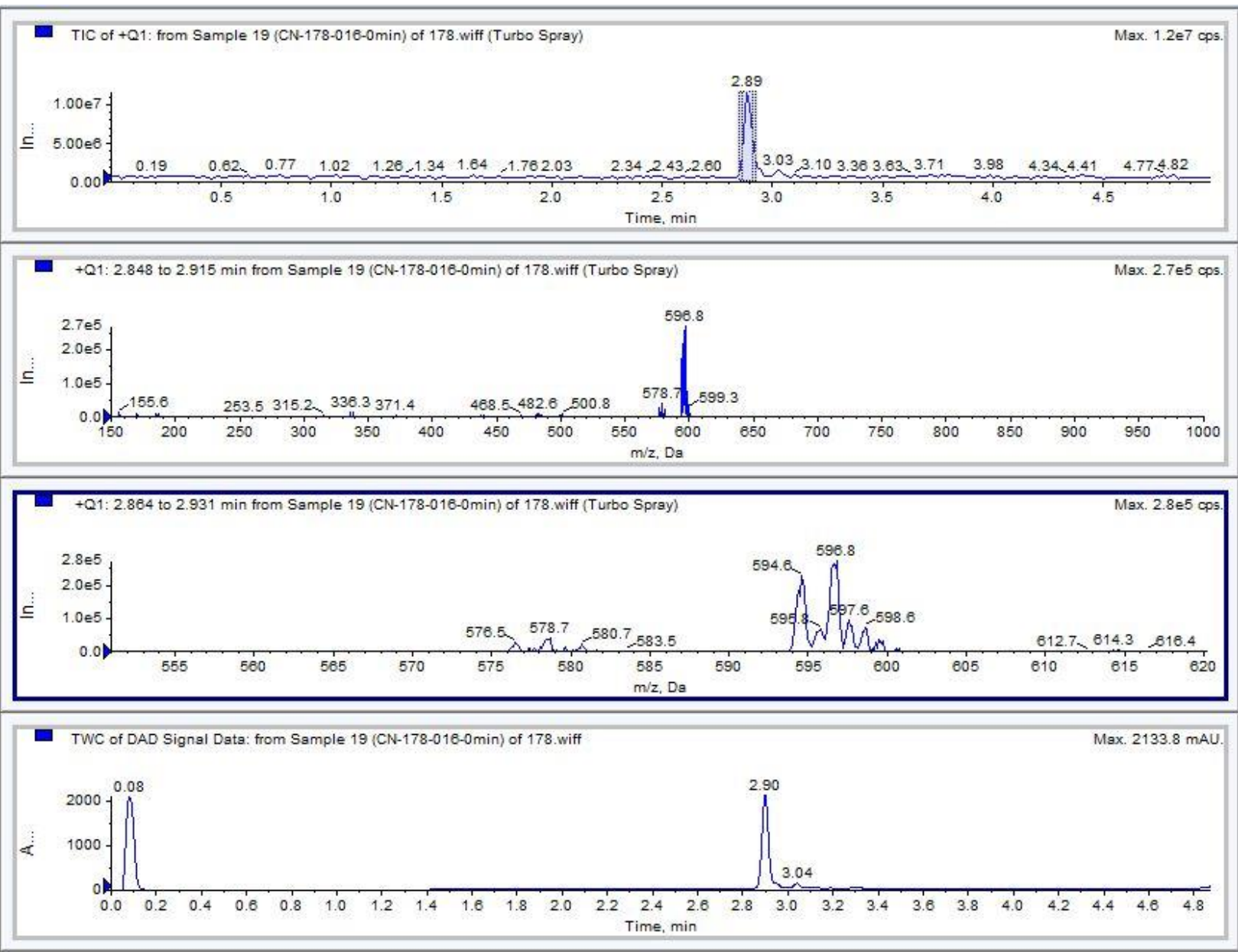


LCMS of 19 (separately synthesized and characterized by LCMS and NMR):<smiles></smiles>

19

Chemical Formula: $\mathrm{C}_{24} \mathrm{H}_{17} \mathrm{BrClNO}_{4}$

Exact Mass: 497.0029

TIC of + Q1: from Sample 20 (CN-178-eFT-1622) of 178.wiff (Turbo Spray)

Max. 8.5e6 cps.

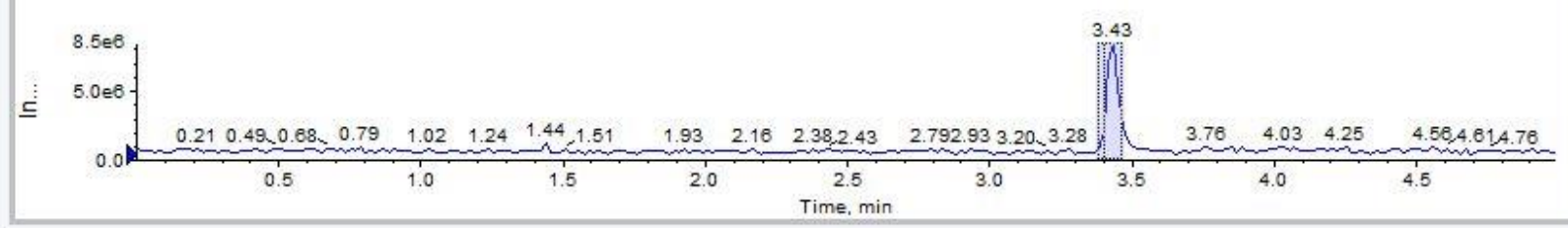

$+Q 1: 3.384$ to 3.487 min from Sample 20 (CN-178-eFT-1622) of 178.wiff (Turbo Spray)

Max. $1.8 \mathrm{e} 5 \mathrm{cps}$

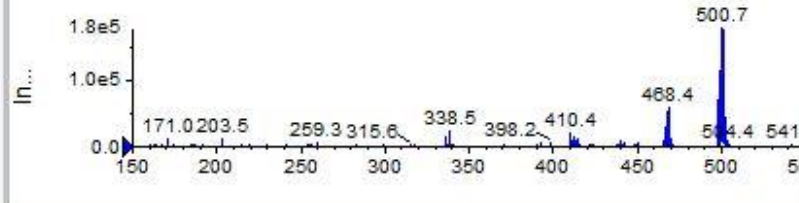

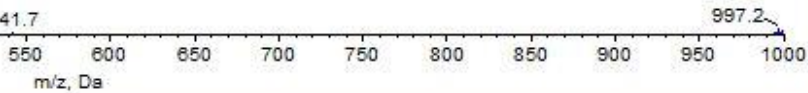

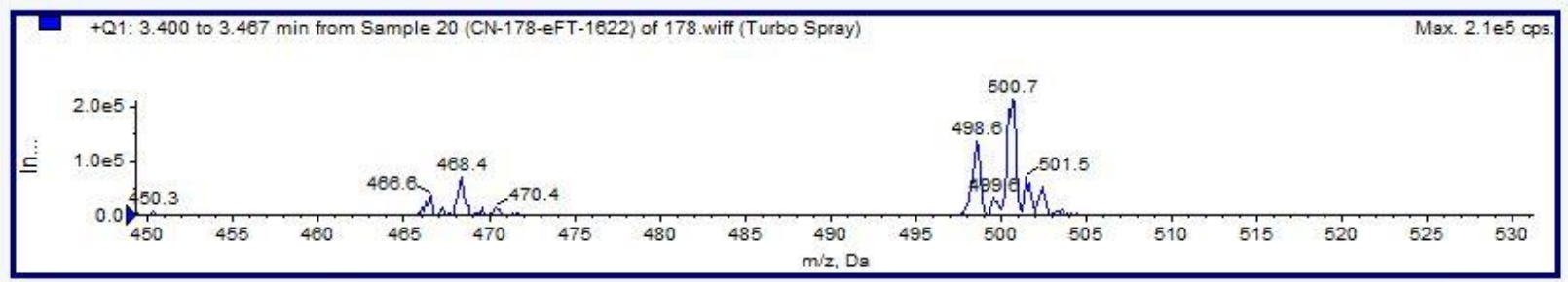

TWC of DAD Signal Data: from Sample 20 (CN-178-eFT-1822) of 178.wiff Max. 2552.2 mAL. 255
$\vdots \quad 200$
$\times \quad 100$

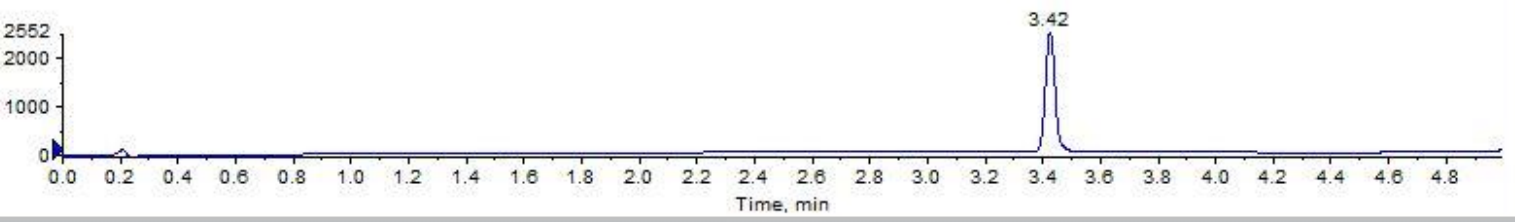


LCMS of 20 (separately synthesized and characterized by LCMS and NMR):<smiles>COC(=O)C1=C[C@]2(O)c3ncc(Cl)cc3O[C@@]2(c2ccc(Br)cc2)C1c1ccccc1</smiles>

20

Chemical Formula: $\mathrm{C}_{24} \mathrm{H}_{17} \mathrm{BrClNO}_{4}$

Exact Mass: 497.0029

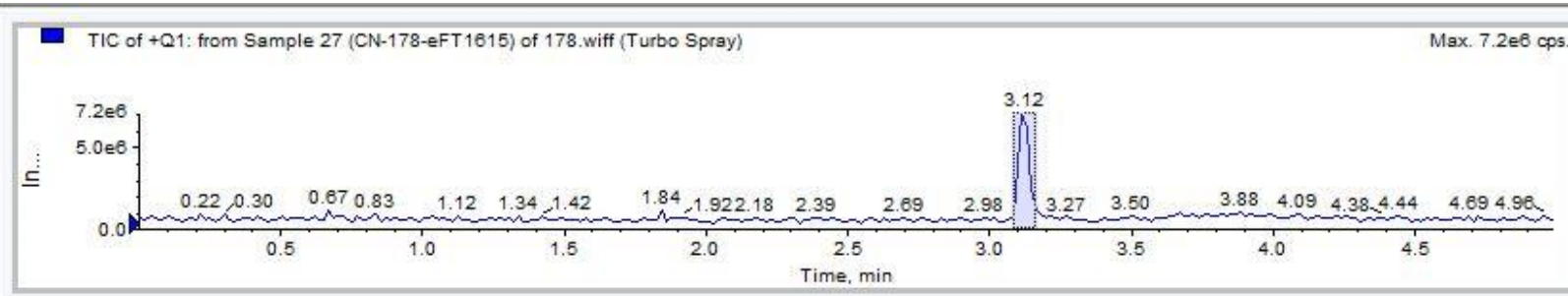

+Q1: 3.082 to $3.188 \mathrm{~min}$ from Sample 27 (CN-178-eFT 1615) of 178. wiff (Turbo Spray)

Mgx. $1.7 \mathrm{e} 5 \mathrm{cps}$

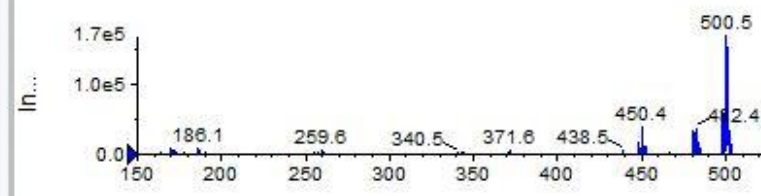

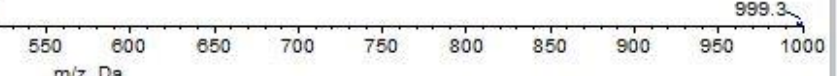

$+\mathrm{Q} 1: 3.082$ to $3.168 \mathrm{~min}$ from Sample 27 (CN-178-eFT1615) of 178.wiff (Turbo Spray)

Max. $1.7 \mathrm{e} 5 \mathrm{cps}$

$\subseteq \quad 1.0 e 5-\frac{3.7 e 5}{370}$

(1)

- TWC of DAD Signal Data: from Sample 27 (CN-178-eFT1615) of 178.wiff

Max. $2273.0 \mathrm{mAU}$

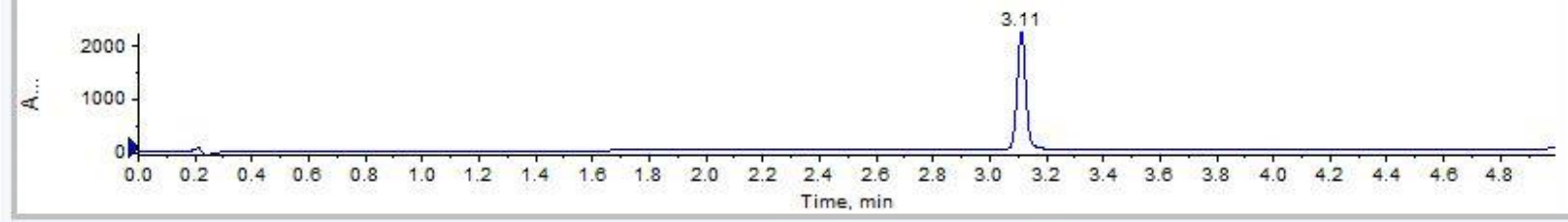


LCMS of 21 (product):<smiles>CO[C@H]1[C@@H](c2ccccc2)[C@@]2(c3ccc(Br)cc3)Oc3cc(Cl)cnc3[C@]2(O)[C@@H]1C#N</smiles>

Chemical Formula: $\mathrm{C}_{25} \mathrm{H}_{18} \mathrm{BrClN}_{2} \mathrm{O}_{4}$

Exact Mass: 524.0138

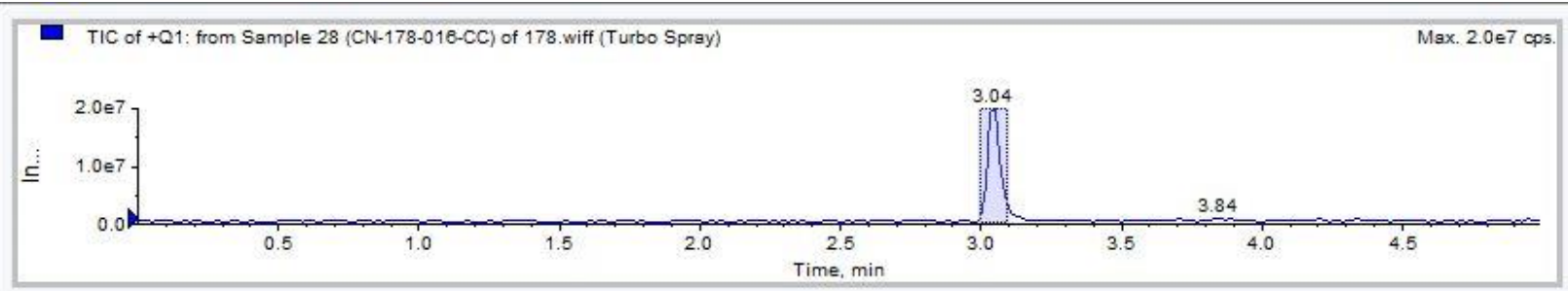

+Q1: 2.998 to 3.099 min from Sample 28 (CN-178-018-CC) of 178 wiff (Turbo Spray)

Max. 4.3e5 cps.

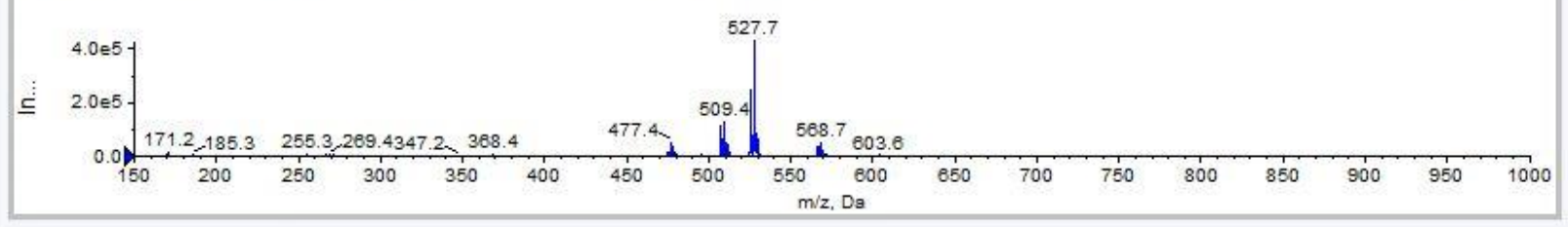

W + Q1: 2.998 to 3.099 min from Sample 28 (CN-178-018-CC) of 178.wiff (Turbo Spray)

Max. 4.3e5 cps

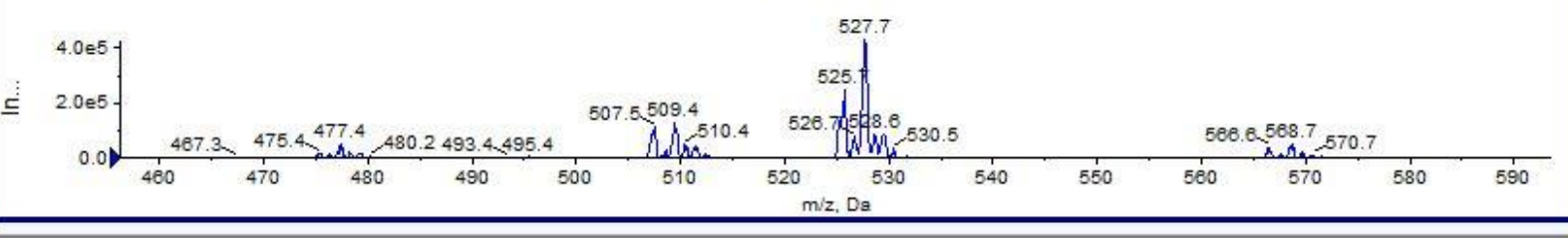

- TWC of DAD Signal Data: from Sample $28(\mathrm{CN}-178-016-\mathrm{CC})$ of 178 .wiff

Max. 3199.1 mAU.

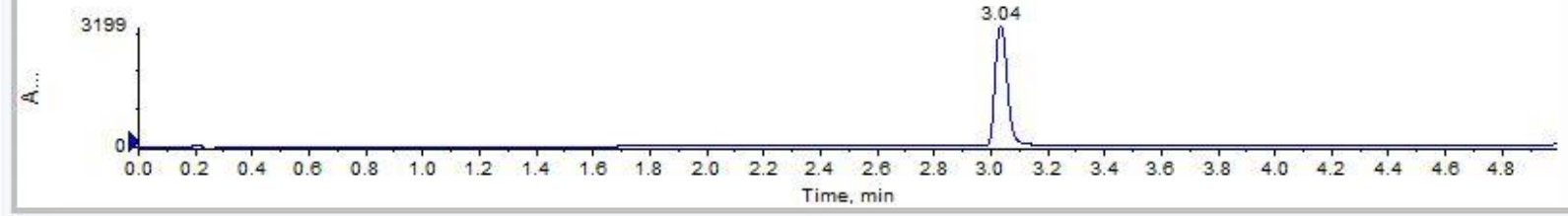




\subsubsection{Synthesis of compound $\mathbf{2 6}$}

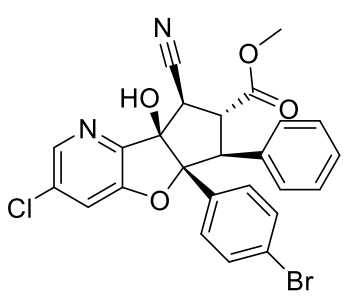

21

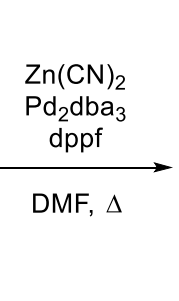

$\frac{\mathrm{Me}_{2} \mathrm{NH}, \mathrm{EDC}, \mathrm{HOBt}}{\text { DIPEA, DCM }}$

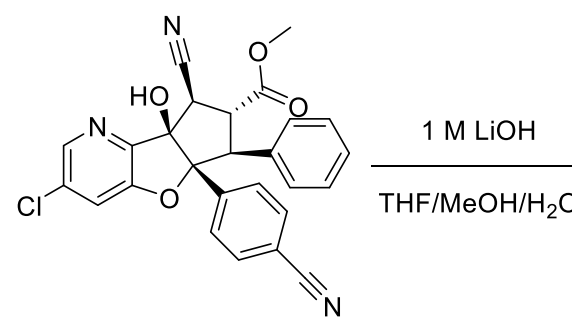

S4

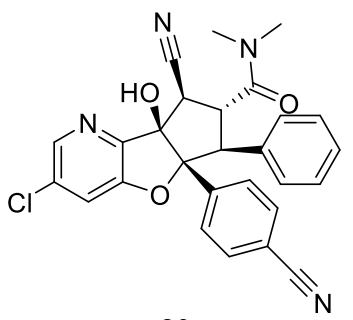

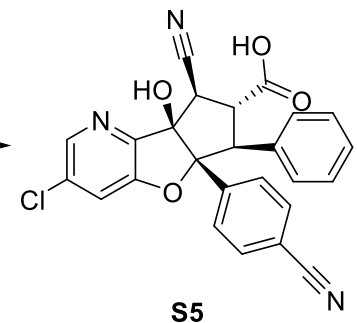

S5

26

$r a c$-methyl (5a $R, 6 S, 7 R, 8 R, 8 \mathrm{a} R)$-3-chloro-8-cyano-5a-(4-cyanophenyl)-8a-hydroxy-6-phenyl5a,7,8,8a-tetrahydro-6H-cyclopenta[4,5]furo[3,2-b]pyridine-7-carboxylate (S4)<smiles>COC(=O)[C@H]1[C@@H](C#N)[C@]2(O)c3ncc(Cl)cc3O[C@@]2(c2ccc(C#N)cc2)[C@H]1c1ccccc1</smiles>

A suspension of rac-methyl $(5 \mathrm{a} R, 6 S, 7 R, 8 R, 8 \mathrm{a} R)-5 \mathrm{a}-(4-$ bromophenyl)-3-chloro-8-cyano-8a-hydroxy-6-phenyl-7,8-dihydro6H-cyclopenta[4,5]furo[1,2-b]pyridine-7-carboxylate (21) (184 mg, $0.350 \mathrm{mmol}$ ), zinc cyanide (247 mg, $2.10 \mathrm{mmol})$, zinc (23 mg, 0.35 $\mathrm{mmol}), \mathrm{Pd}_{2}(\mathrm{dba})_{3}(32 \mathrm{mg}, 0.035 \mathrm{mmol})$ and dppf (38.8 mg, 0.0700 $\mathrm{mmol})$ in DMF $(3 \mathrm{~mL})$ and water $(0.3 \mathrm{~mL})$ was purged with argon for $5 \mathrm{~min}$. The reaction was stirred at $120^{\circ} \mathrm{C}$ for $6 \mathrm{~h}$ in a heating block. The resulting mixture was cooled to room temperature, diluted with DCM and filtered through a pad of celite. The filtrate was washed with water. The combined organics were dried over magnesium sulfate, filtered and concentrated. The crude product was purified via flash chromatography (silica, ethyl acetate/hexanes $=0-30 \%$ ) to afford S4 as a white solid. HRMS (ESI) m/z: $[\mathrm{M}+\mathrm{H}]^{+} \mathrm{Calcd}$ for $\mathrm{C}_{26} \mathrm{H}_{19} \mathrm{ClN}_{3} \mathrm{O}_{4}{ }^{+}$472.1059; Found 472.1053; ${ }^{1} \mathrm{H}$ NMR (400 MHz, DMSO-d6) $\delta / \mathrm{ppm}=8.38(\mathrm{~d}$, $J=2.0 \mathrm{~Hz}, 1 \mathrm{H}), 7.90(\mathrm{~d}, J=2.0 \mathrm{~Hz}, 1 \mathrm{H}), 7.68-7.63(\mathrm{~m}, 2 \mathrm{H}), 7.38(\mathrm{~d}, J=8.3 \mathrm{~Hz}, 2 \mathrm{H}), 7.11-7.05$ (m, 3H), 6.99-6.92 (m, 2H), 6.89 (s, 1H), 4.33-4.24 (m, 2H), 3.89-3.80 (m, 1H), $3.54(\mathrm{~s}, 3 \mathrm{H}) ;{ }^{13} \mathbf{C}$ NMR (101 MHz, DMSO-d6) $\delta$ / ppm = 170.3, 150.9, 149.4, 142.3, 139.3, 134.6, 132.3, 131.0, $128.4,127.9,127.8,127.2,119.5,118.6,117.2,110.1,101.3,84.0,57.1,52.6,47.4,41.2$. 
rac-(5a $R, 6 S, 7 R, 8 R, 8 \mathrm{a} R)$-3-chloro-8-cyano-5a-(4-cyanophenyl)-8a-hydroxy-6-phenyl-

\section{5a,7,8,8a-tetrahydro-6H-cyclopenta[4,5]furo[3,2-b]pyridine-7-carboxylic acid (S5)}

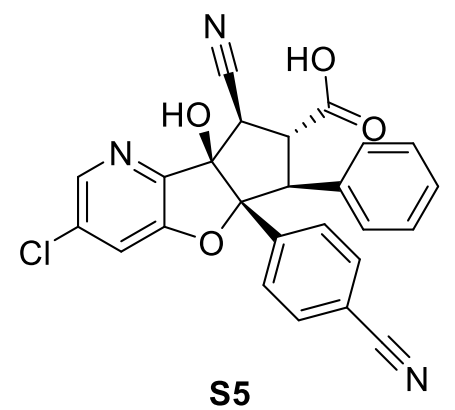

S5

To a solution of rac-methyl $(5 \mathrm{a} R, 6 S, 7 R, 8 R, 8 \mathrm{a} R)$-3-chloro-8-cyano-

5a-(4-cyanophenyl)-8a-hydroxy-6-phenyl-5a,7,8,8a-tetrahydro-6Hcyclopenta[4,5]furo[3,2-b]pyridine-7-carboxylate (S4) (15 mg, 0.032 mmol) in tetrahydrofuran $(1 \mathrm{~mL})$ and methanol $(1 \mathrm{~mL})$ was added 2 M lithium hydroxide ( $1 \mathrm{~mL}, 2 \mathrm{mmol})$. The reaction was stirred at room temperature for $5 \mathrm{~min}$. The reaction was acidified with $1 \mathrm{M}$ hydrochloric acid and the organic volatiles were evaporated. The mixture was diluted with water and extracted with dichloromethane. The combined organics were dried over magnesium sulfate, filtered and concentrated. The crude product $\mathbf{S 5}$ was used for next step without further purification. Yield: $15 \mathrm{mg}$, crude; HRMS (ESI) m/z: $[\mathrm{M}+\mathrm{H}]^{+}$Calcd for $\mathrm{C}_{25} \mathrm{H}_{17} \mathrm{ClN}_{3} \mathrm{O}_{4}{ }^{+} 458.0902$; Found 458.0888.

rac-(5a $R, 6 S, 7 R, 8 R, 8 \mathrm{a} R)-3-c h l o r o-8-c y a n o-5 a-(4-c y a n o p h e n y l)-8 a-h y d r o x y-N, N-d i m e t h y l-6-$ phenyl-5a,7,8,8a-tetrahydro-6H-cyclopenta[4,5]furo[3,2-b]pyridine-7-carboxamide (26)

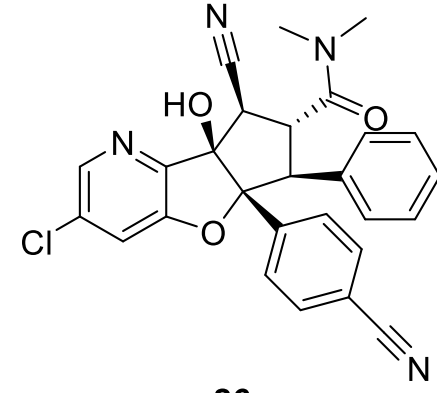

26

To a solution of crude rac- $(5 \mathrm{a} R, 6 S, 7 R, 8 R, 8 \mathrm{a} R)-3$-chloro-8-cyano-5a(4-cyanophenyl)-8a-hydroxy-6-phenyl-5a,7,8,8a-tetrahydro-6Hcyclopenta[4,5]furo[3,2-b]pyridine-7-carboxylic acid (S5) (30 mg), $\mathrm{N}$-(3-dimethylaminopropyl)-N'-ethylcarbodiimide hydrochloride (39 $\mathrm{mg}, 0.20 \mathrm{mmol})$, 1-hydroxybenzotriazole hydrate $(31 \mathrm{mg}, 0.20 \mathrm{mmol})$ in dichloromethane $(4 \mathrm{~mL})$ at $0 \quad{ }^{\circ} \mathrm{C}$ were added $\mathrm{N}, \mathrm{N}$ diisopropylethylamine $(0.08 \mathrm{~mL}, \quad 0.46 \mathrm{mmol})$ and $\mathrm{N}$ methylmethanamine hydrochloride $(11 \mathrm{mg}, 0.13 \mathrm{mmol})$. The reaction was stirred at room temperature overnight. The mixture was diluted with dichloromethane and washed with aqueous saturated sodium bicarbonate solution. The combined organics were dried over magnesium sulfate, filtered and concentrated. The crude product was purified via column chromatography (silica, ethyl acetate/hexane $=0-60 \%)$, followed by preparative HPLC (C18, MeCN/water+0.1\% TFA) to afford rac-(5aR,6S,7R,8R,8aR)-3-chloro-8-cyano-5a-(4-cyanophenyl)-8a-hydroxy-N,N-dimethyl-6phenyl-5a,7,8,8a-tetrahydro-6H-cyclopenta[4,5]furo[3,2-b]pyridine-7-carboxamide (26) as a white solid. Yield: $13.7 \mathrm{mg}$ (0.0277 mmol, 43\% over two steps); HRMS (ESI) m/z: [M+H] $]^{+}$Calcd 
for $\mathrm{C}_{27} \mathrm{H}_{22} \mathrm{ClN}_{4} \mathrm{O}_{3}{ }^{+}$485.1375; Found 485.1359; ${ }^{1} \mathrm{H}$ NMR (400 MHz, DMSO-d6) $\delta / \mathrm{ppm}=8.37$ (d, $J=2.0 \mathrm{~Hz}, 1 \mathrm{H}), 7.90(\mathrm{~d}, J=2.0 \mathrm{~Hz}, 1 \mathrm{H}), 7.73-7.65(\mathrm{~m}, 2 \mathrm{H}), 7.48(\mathrm{~d}, J=8.3 \mathrm{~Hz}, 2 \mathrm{H}), 7.11-$ 7.07 (m, 3H), 6.91 (d, $J=0.6 \mathrm{~Hz}, 1 \mathrm{H}), 6.89-6.83(\mathrm{~m}, 2 \mathrm{H}), 4.49$ (dd, $J=12.6,10.4 \mathrm{~Hz}, 1 \mathrm{H}), 3.92$ $(\mathrm{d}, J=12.6 \mathrm{~Hz}, 1 \mathrm{H}), 3.90(\mathrm{dd}, J=10.4,0.6 \mathrm{~Hz}, 1 \mathrm{H}), 3.26(\mathrm{~s}, 3 \mathrm{H}), 2.77(\mathrm{~s}, 3 \mathrm{H}) ;{ }^{13} \mathbf{C}$ NMR $(101$ MHz, d6-DMSO) $\delta / \mathrm{ppm}=168.5,150.9,149.4,142.3,139.6,134.4,132.4,131.1,128.6,127.98$, 127.96, 127.4, 119.5, 118.6, 117.1, 110.2, 101.5, 84.7, 58.8, 44.1, 42.4, 37.3, 35.8. 


\section{1D and 2D NMR spectra}

rac-(5a $R, 6 S, 8 R, 8 \mathrm{aS})$-5a-(4-bromophenyl)-3-chloro-6-phenyl-5a,6,7,8-tetrahydro-8aHcyclopenta[4,5]furo[3,2-b]pyridine-8,8a-diol (9)

${ }^{1} \mathrm{H}-\mathrm{NMR}\left(400 \mathrm{MHz}, \mathrm{d}^{6}\right.$-DMSO)

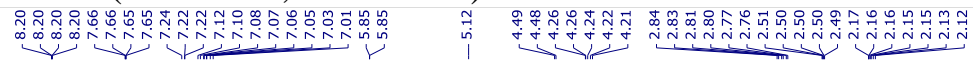

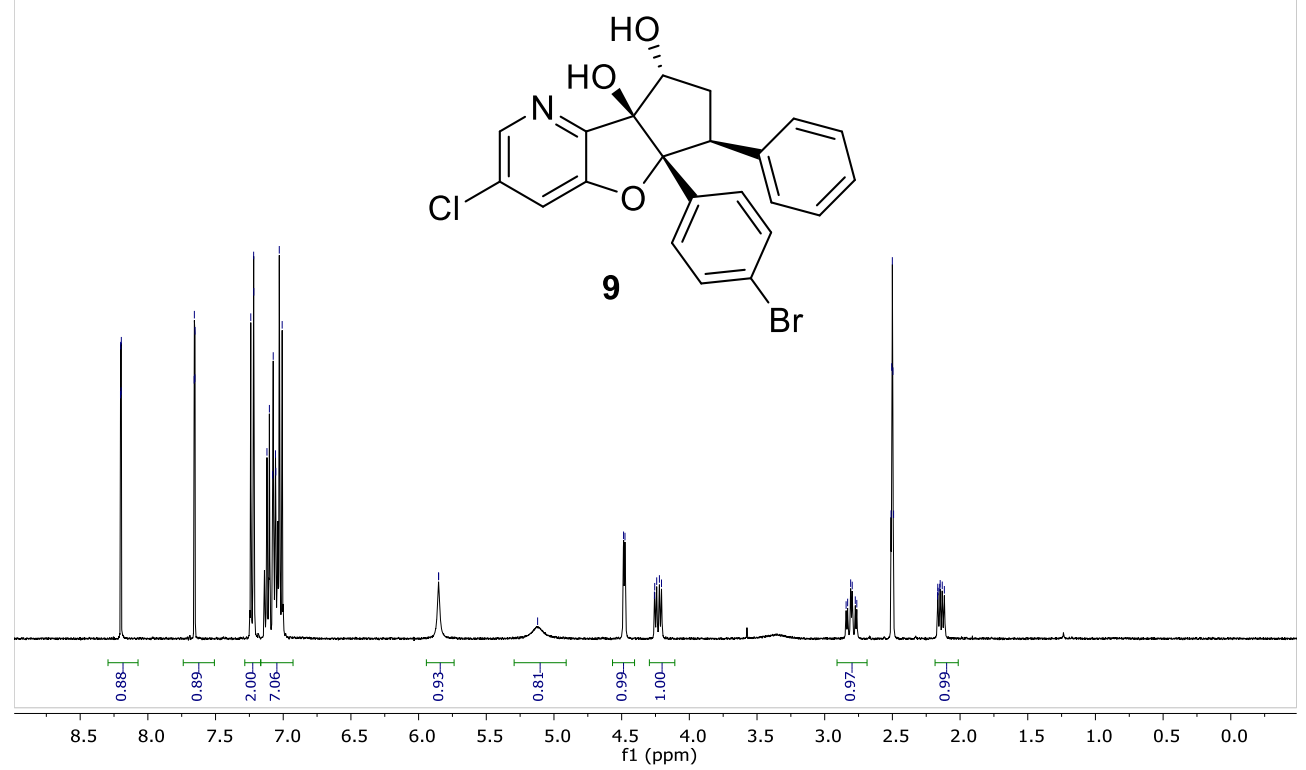

${ }^{13} \mathrm{C}-\mathrm{NMR}\left(101 \mathrm{MHz}, \mathrm{d}^{6}\right.$-DMSO)

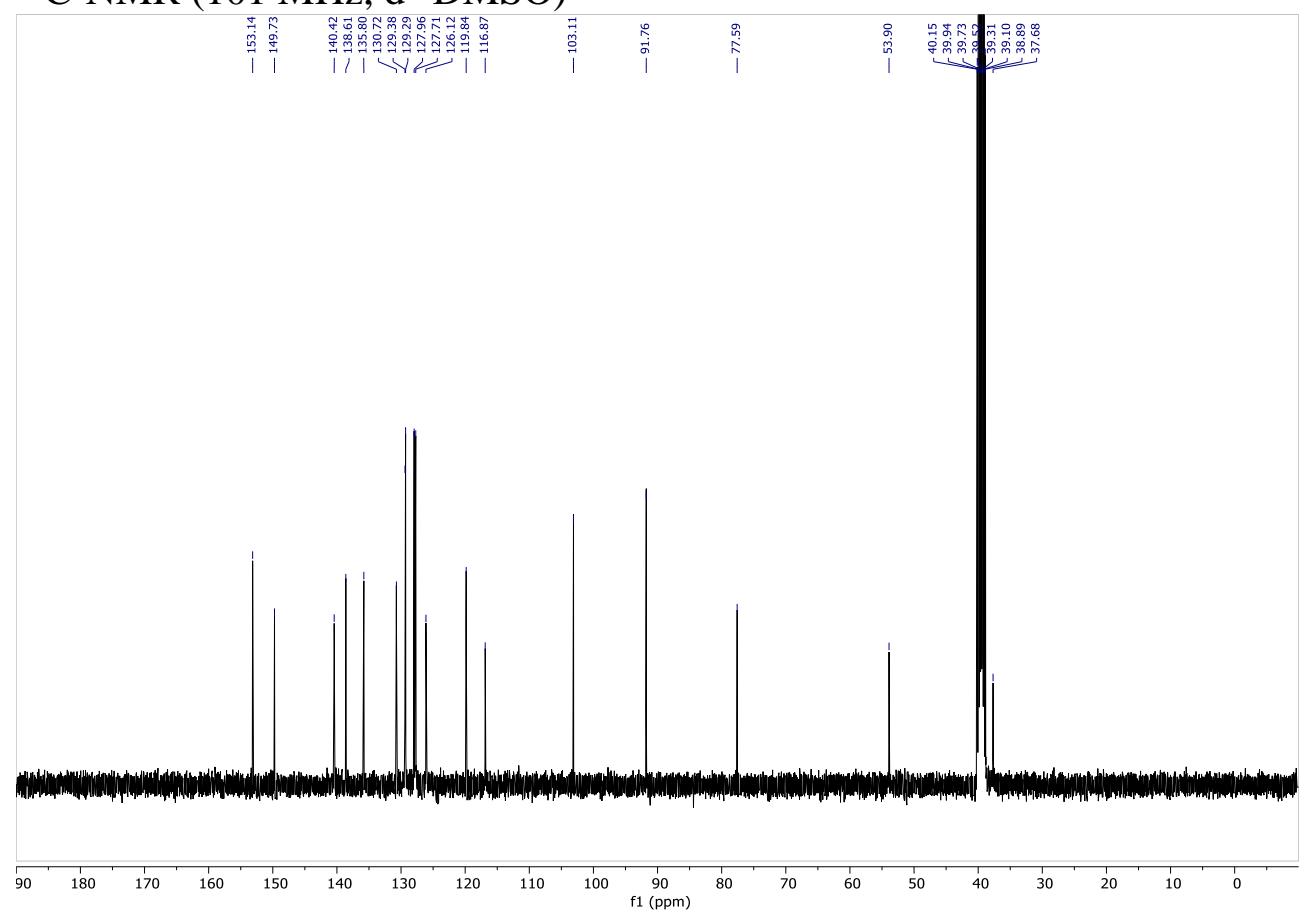


${ }^{1} \mathrm{H},{ }^{1} \mathrm{H}-\mathrm{COSY}\left(\mathrm{d}^{6}\right.$-DMSO)

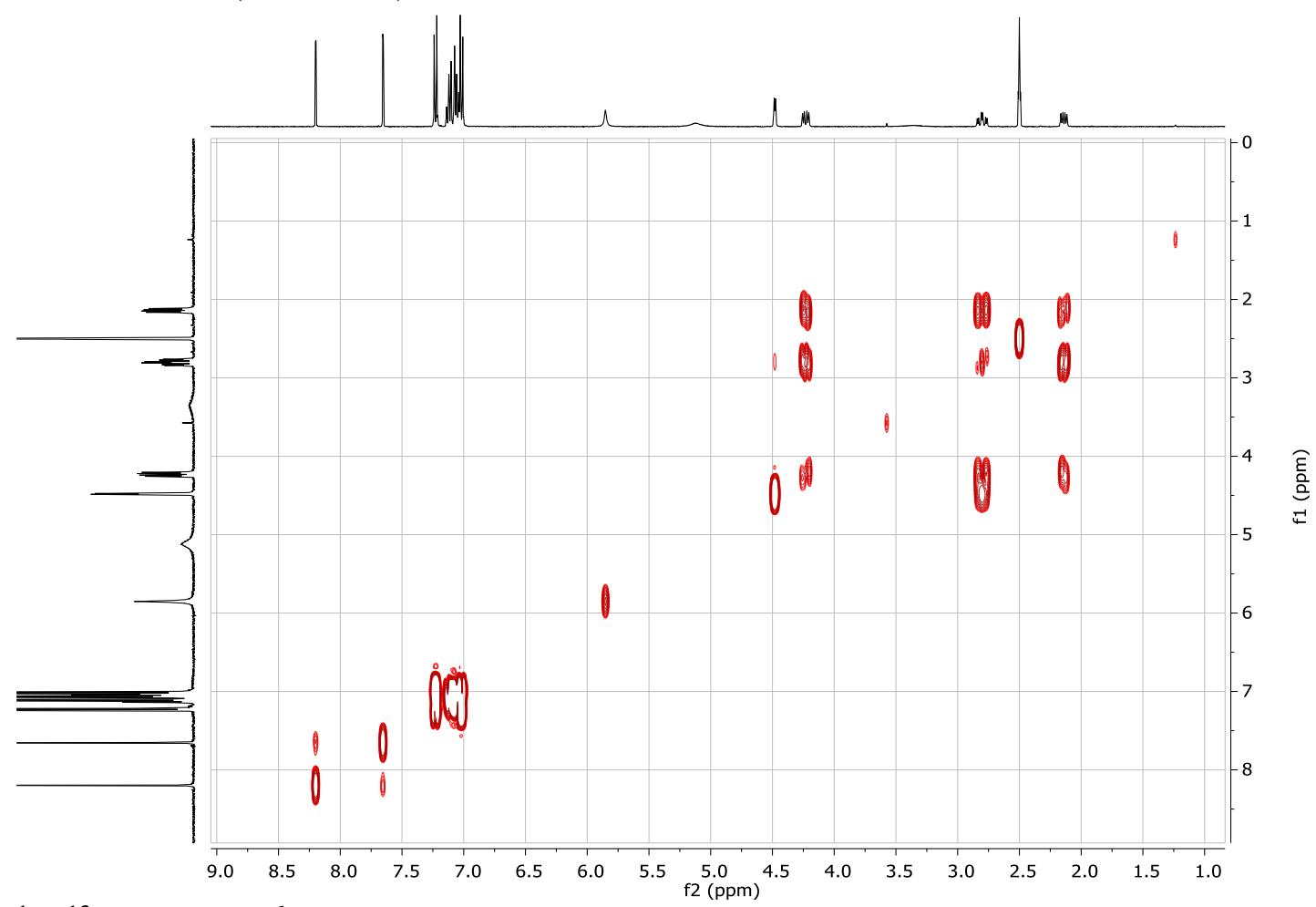

${ }^{1} \mathrm{H},{ }^{13} \mathrm{C}$-HSQC ( $\mathrm{d}^{6}$-DMSO)

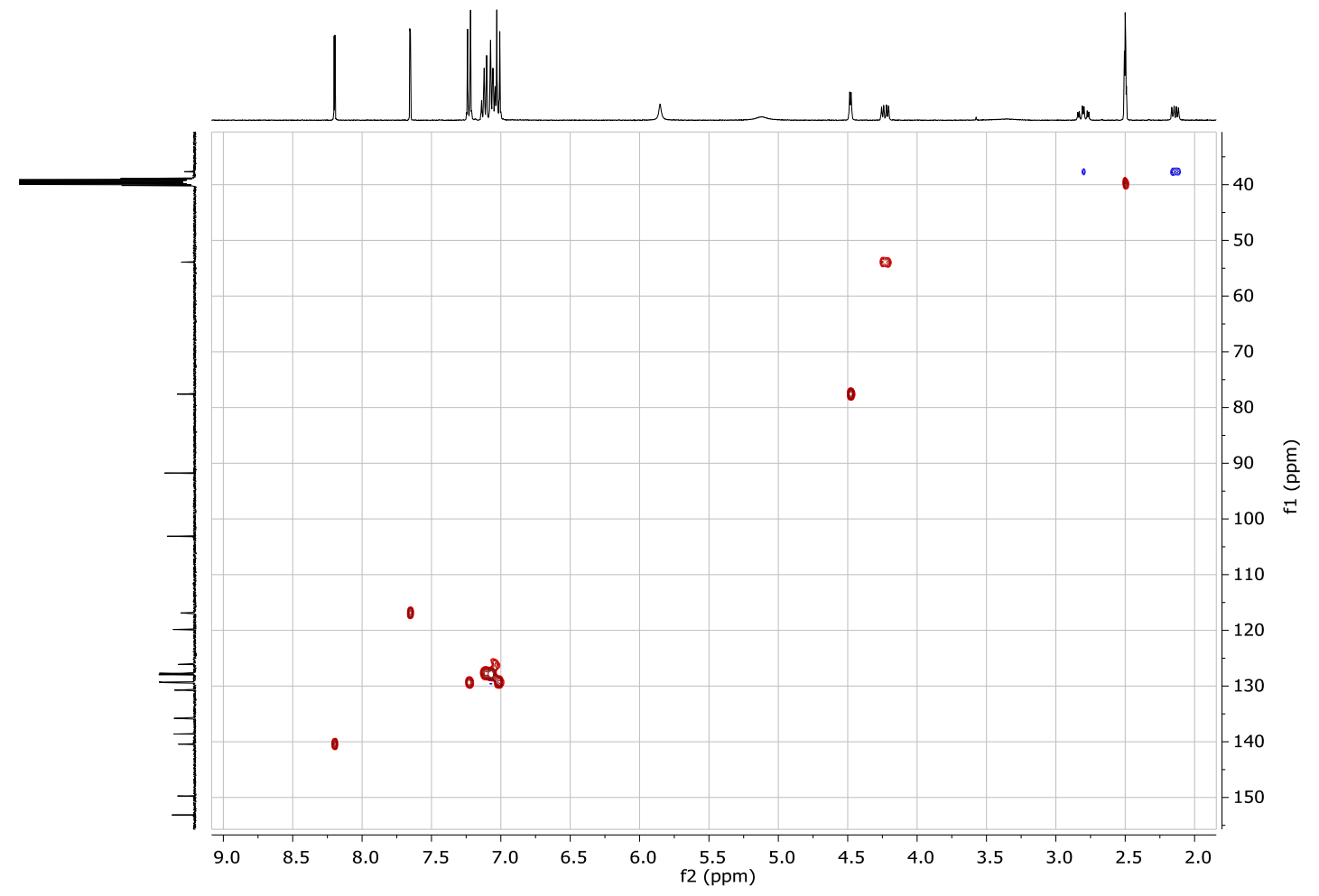




\section{${ }^{1} \mathrm{H},{ }^{1} \mathrm{H}-\mathrm{NOESY}\left(\mathrm{d}^{6}\right.$-DMSO)}

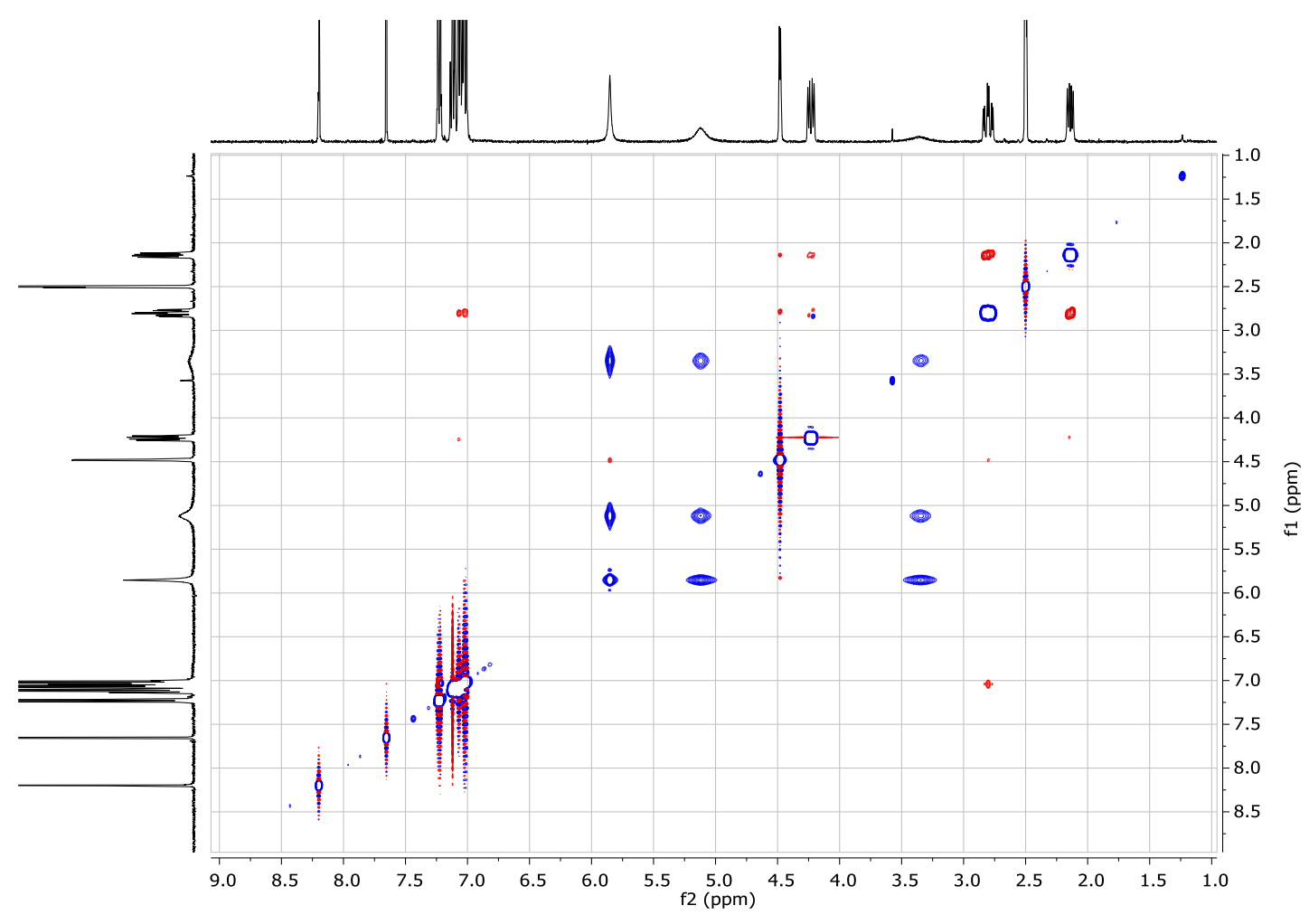


$r a c$-(5a $R, 6 S, 8 R, 8 \mathrm{a} S)-5 \mathrm{a}-(4-b r o m o p h e n y l)-3-c h l o r o-8 a-h y d r o x y-6-p h e n y l-5 a, 7,8,8 \mathrm{a}-$ tetrahydro-6H-cyclopenta[4,5]furo[3,2-b]pyridin-8-yl methanesulfonate (14)

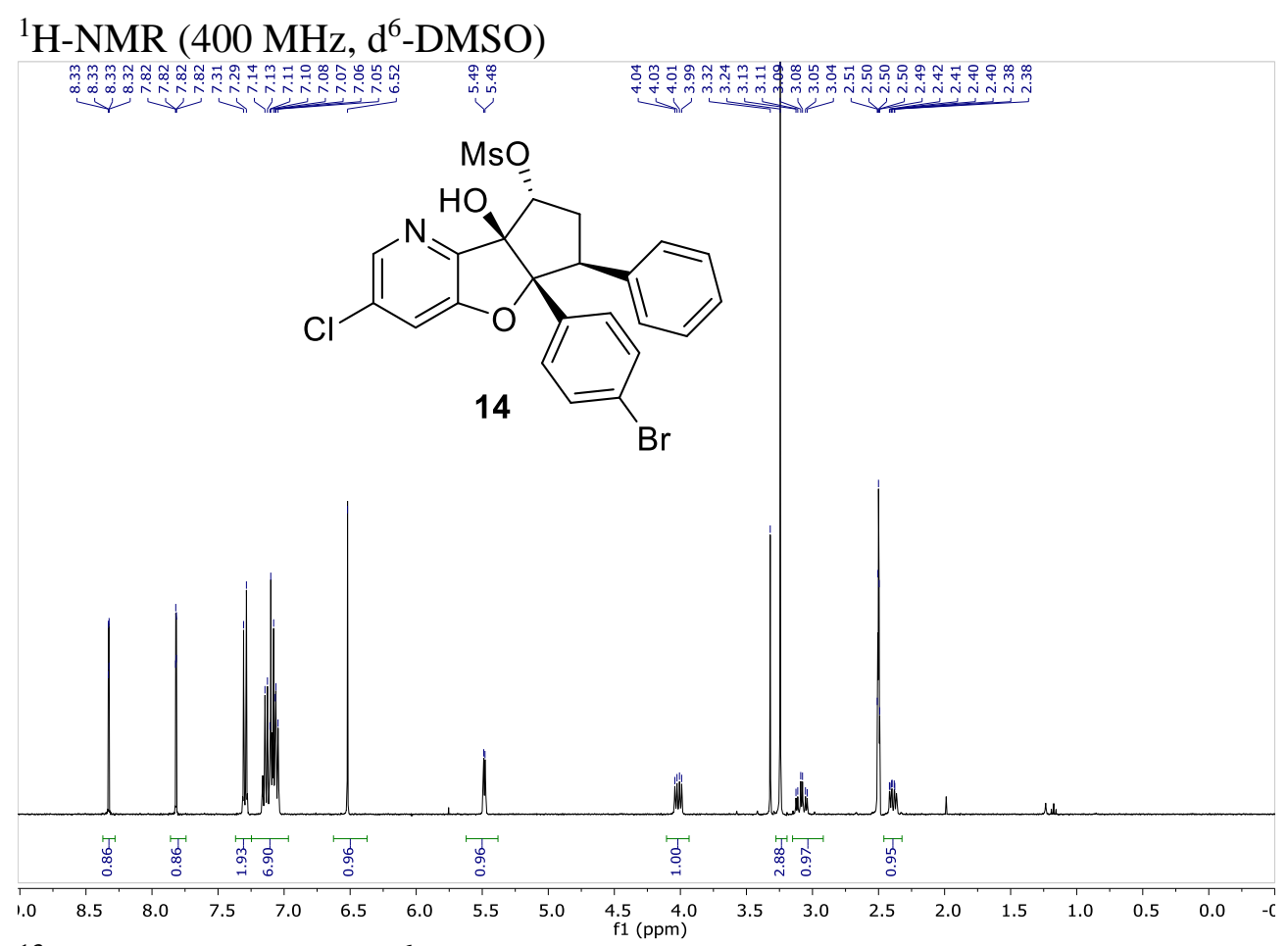

${ }^{13} \mathrm{C}-\mathrm{NMR}\left(101 \mathrm{MHz}, \mathrm{d}^{6}\right.$-DMSO)

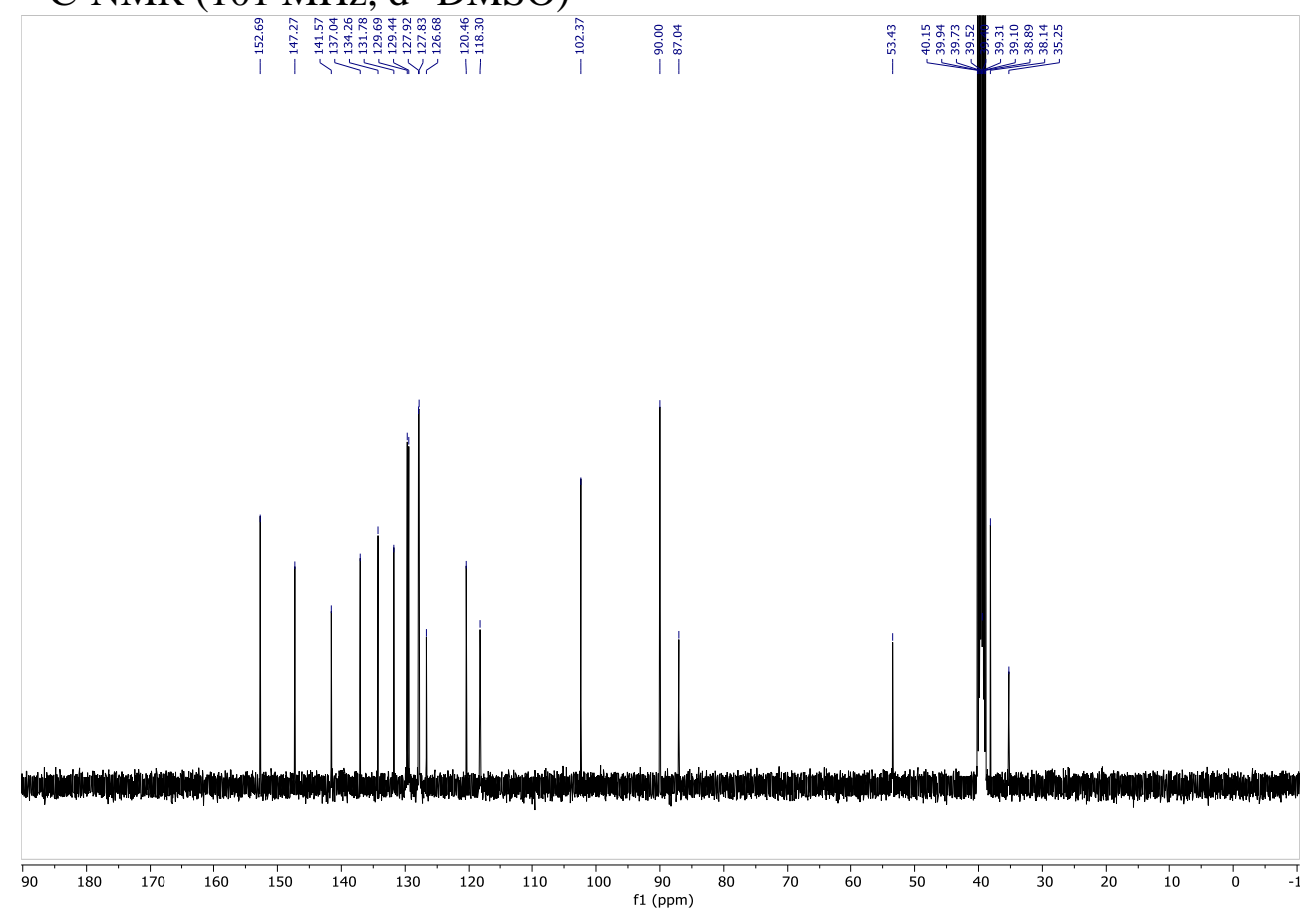


${ }^{1} \mathrm{H},{ }^{1} \mathrm{H}-\mathrm{COSY}$ (d ${ }^{6}$-DMSO)

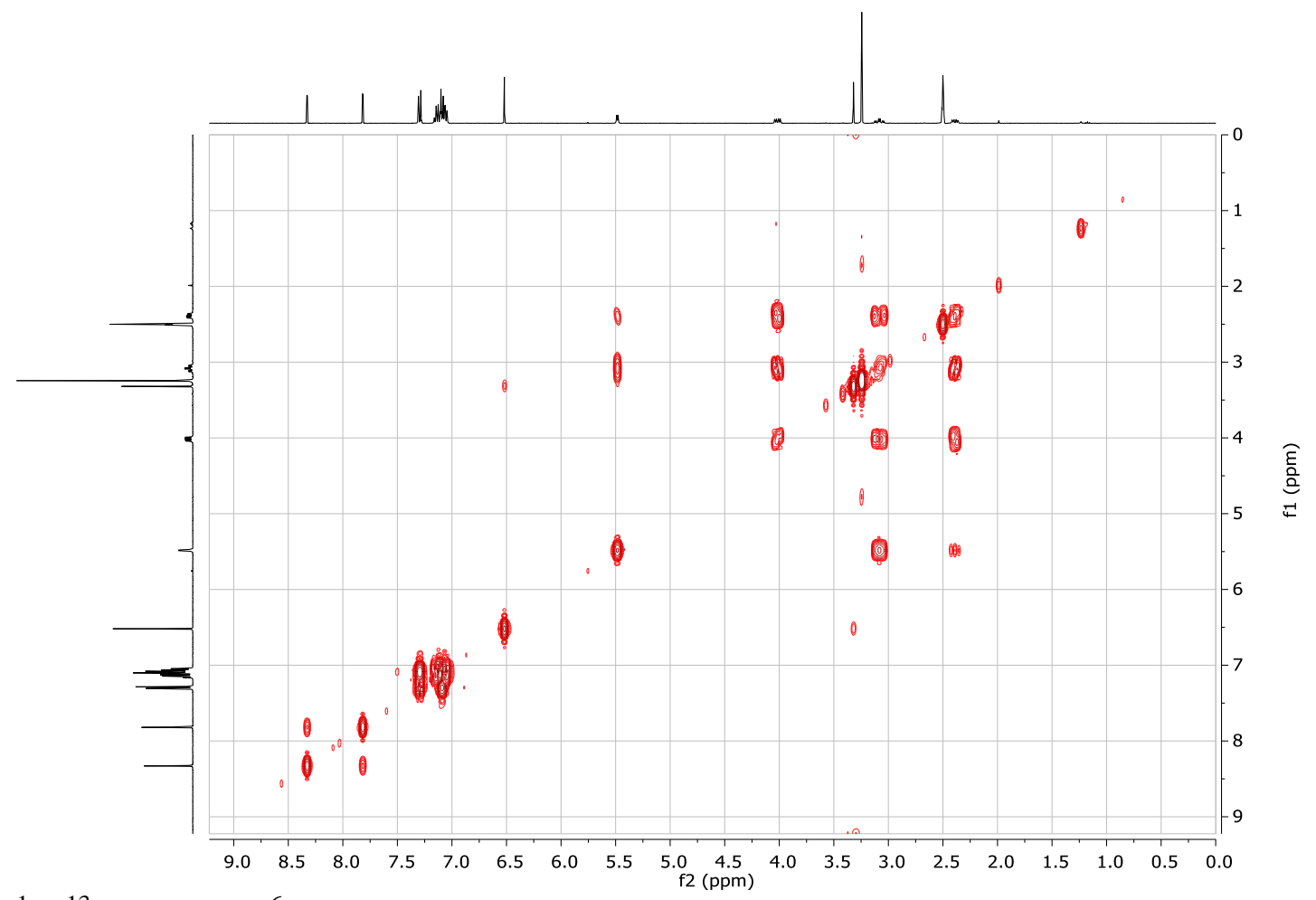

${ }^{1} \mathrm{H},{ }^{13} \mathrm{C}$-HSQC ( $\mathrm{d}^{6}$-DMSO)

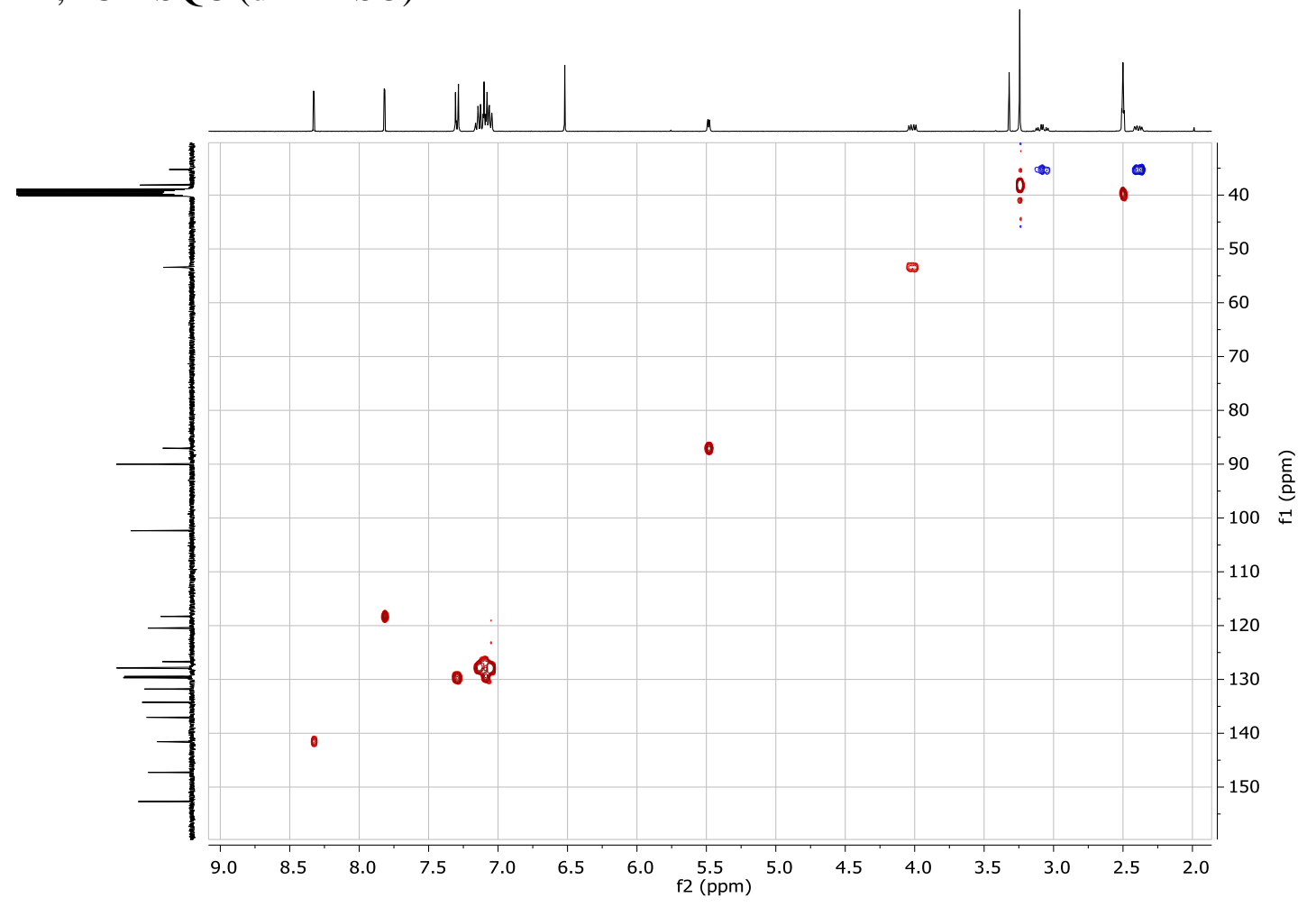


${ }^{1} \mathrm{H},{ }^{1} \mathrm{H}-\mathrm{NOESY}\left(\mathrm{d}^{6}-\mathrm{DMSO}\right)$

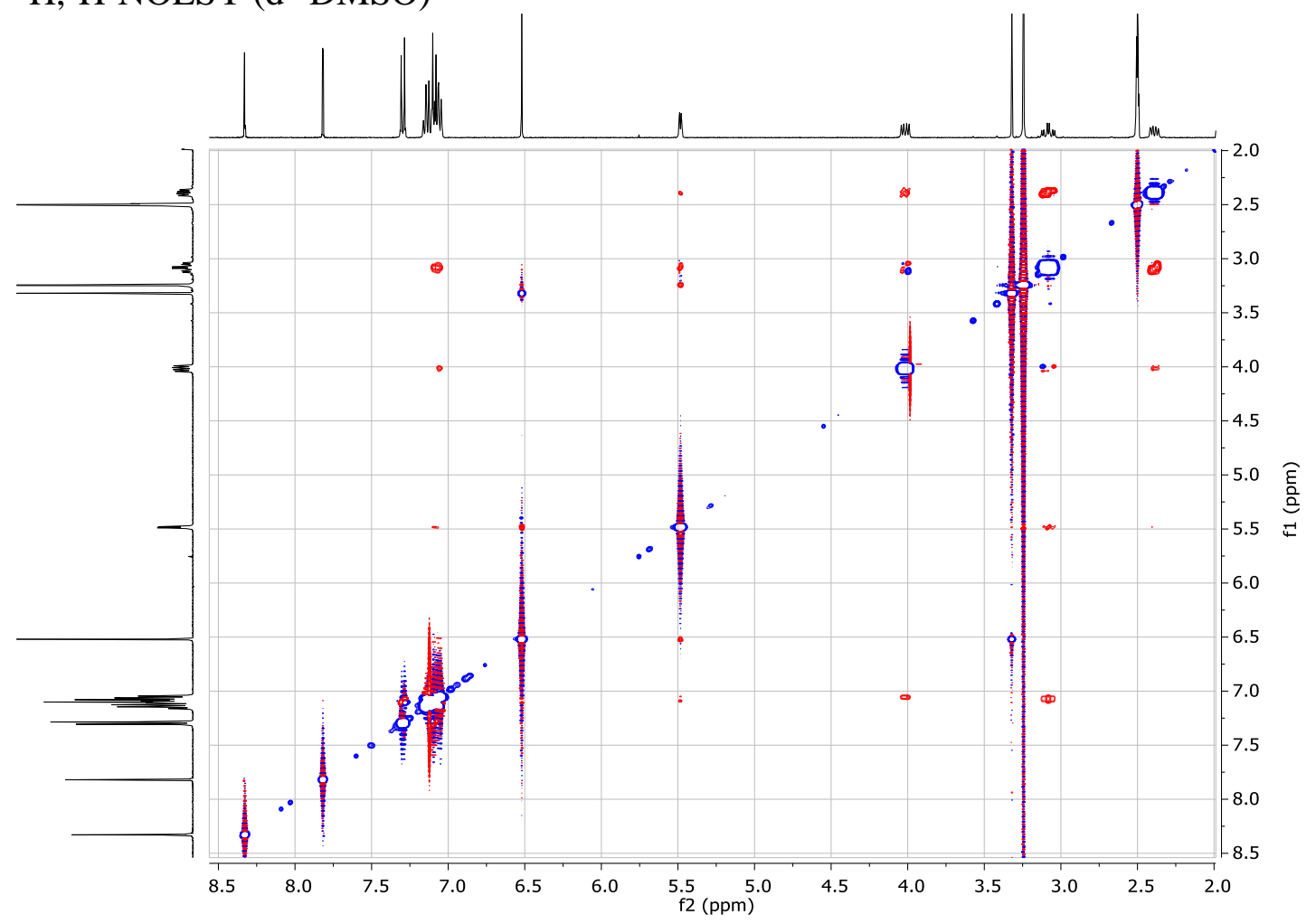


rac-(5a $R, 6 S, 8 S, 8 a R)-5 a-(4-b r o m o p h e n y l)-3-c h l o r o-8 a-h y d r o x y-6-p h e n y l-5 a, 7,8,8 a-$ tetrahydro-6H-cyclopenta[4,5]furo[3,2-b]pyridine-8-carbonitrile (15)

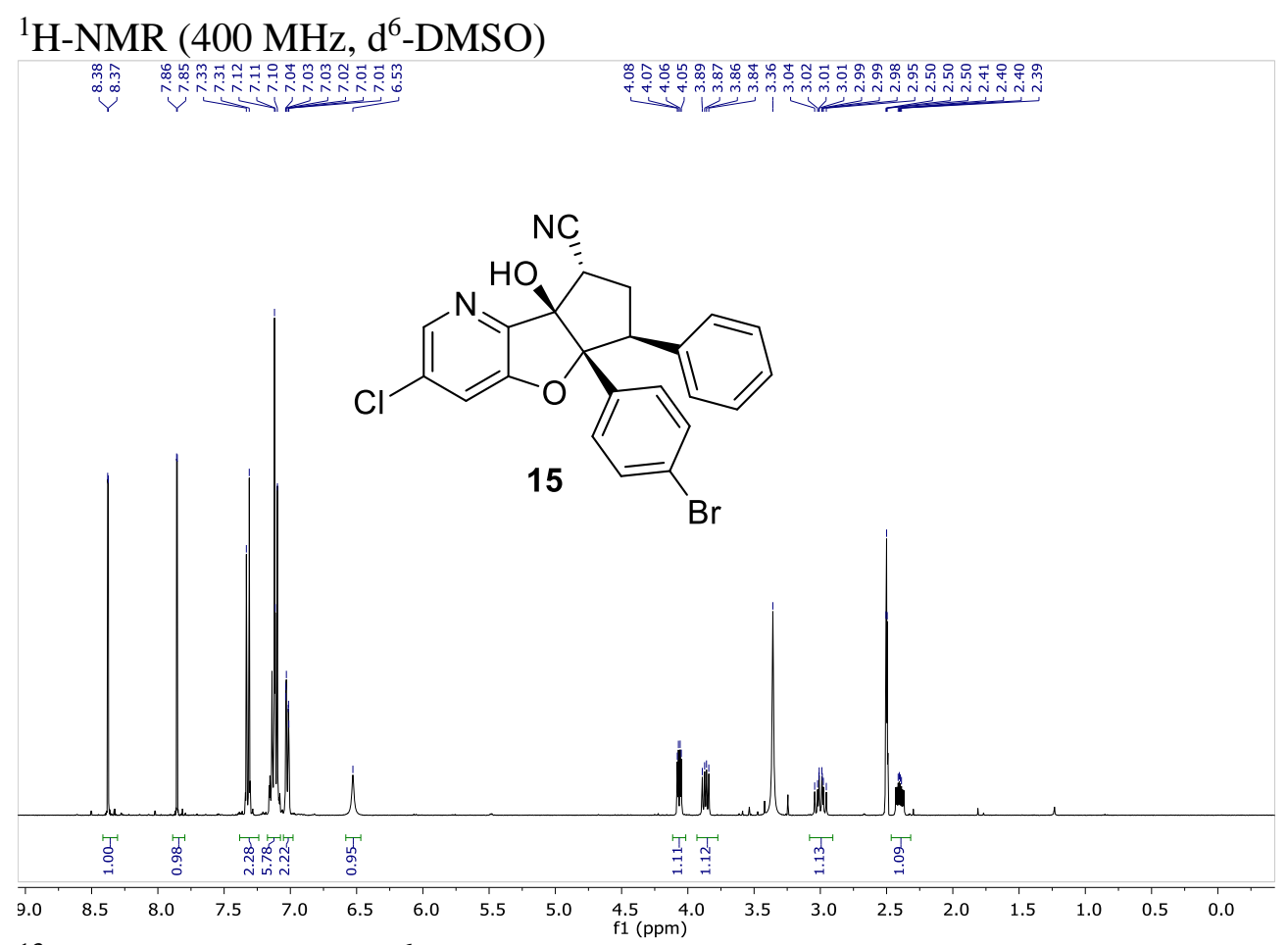

${ }^{13} \mathrm{C}-\mathrm{NMR}$ (101 MHz, d $\mathrm{d}^{6}$-DMSO)

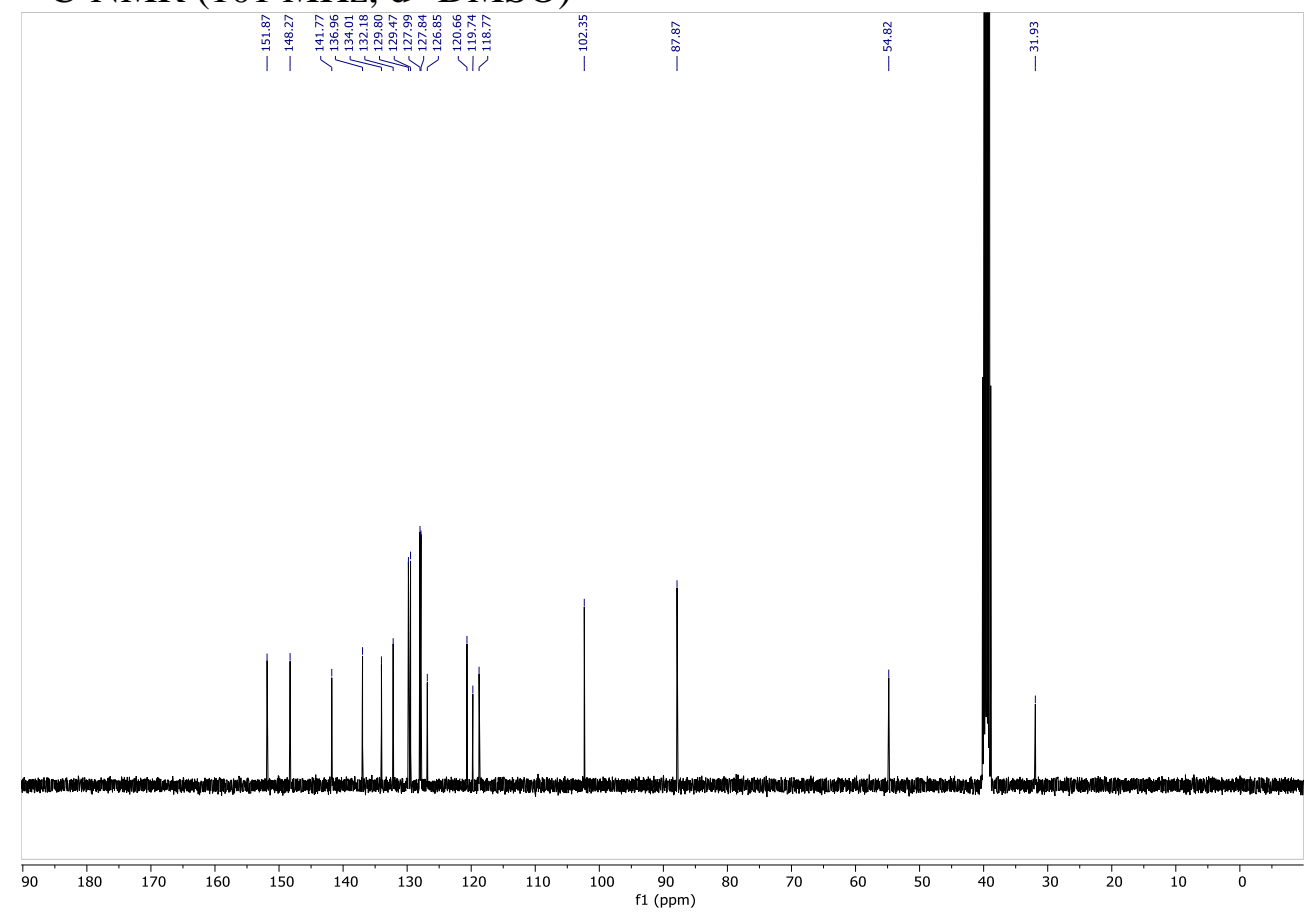


${ }^{1} \mathrm{H},{ }^{1} \mathrm{H}-\mathrm{COSY}\left(\mathrm{d}^{6}\right.$-DMSO)

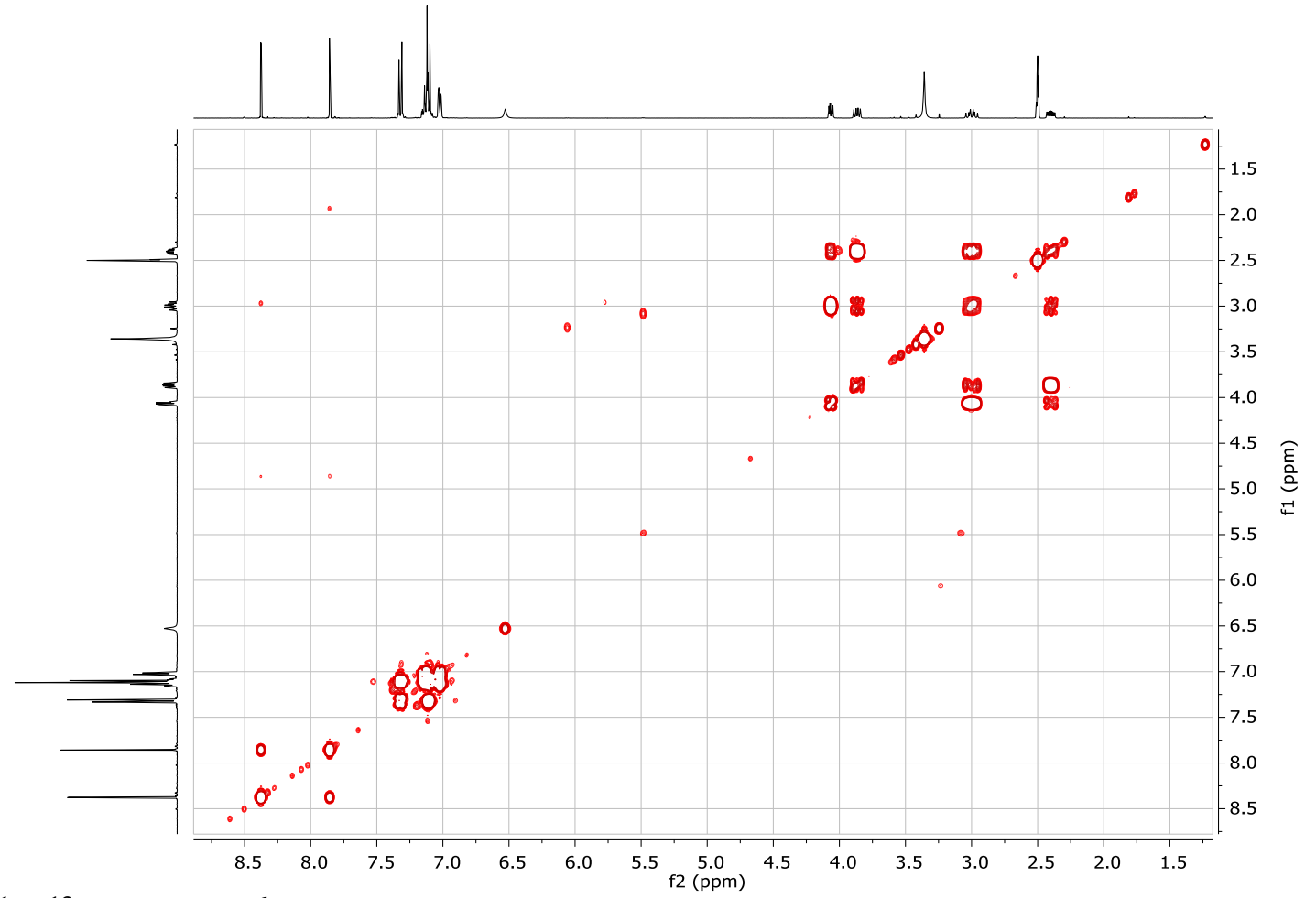

${ }^{1} \mathrm{H},{ }^{13} \mathrm{C}$-HSQC ( $\mathrm{d}^{6}$-DMSO)

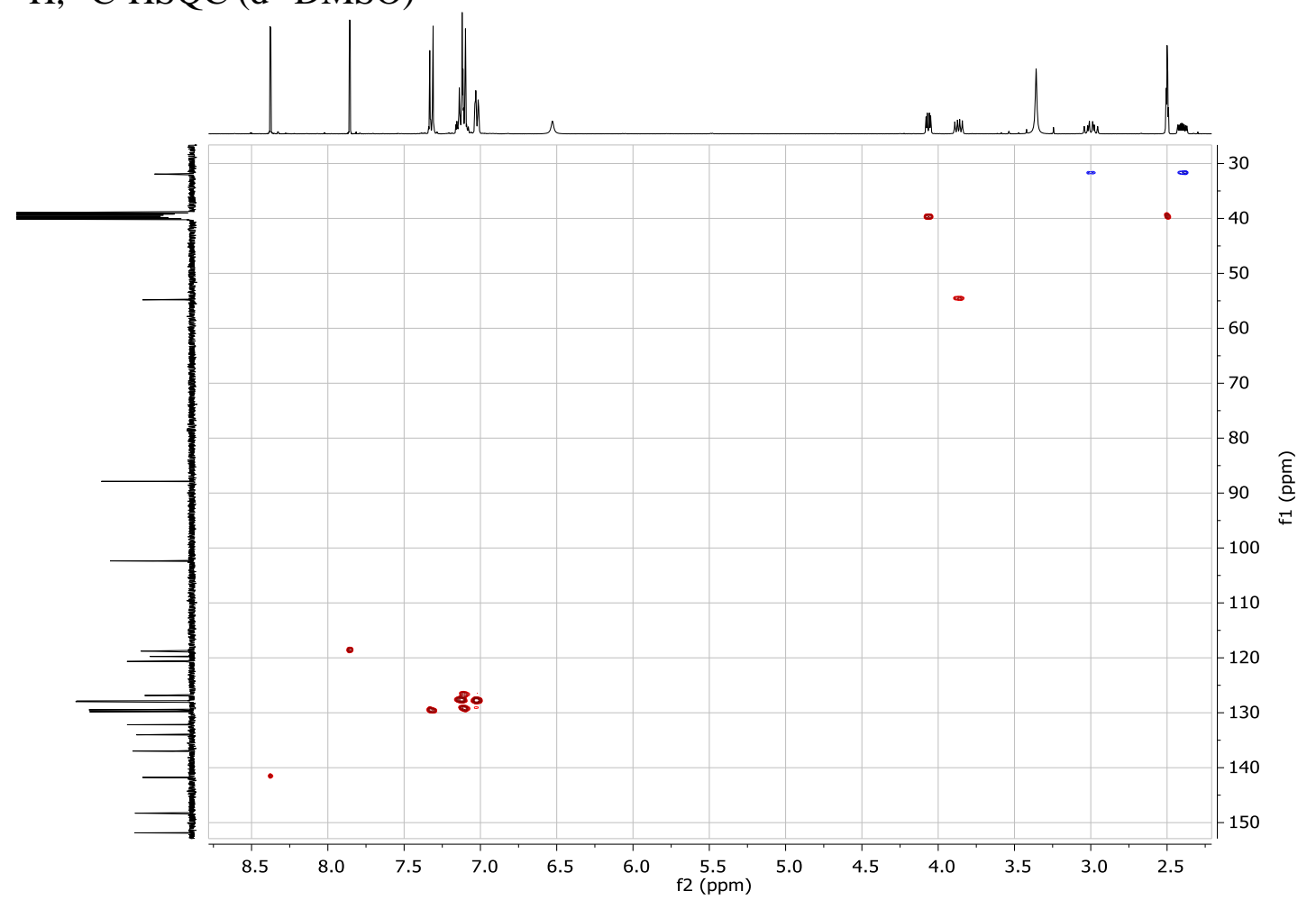


${ }^{1} \mathrm{H},{ }^{13} \mathrm{C}-\mathrm{HMBC}\left(\mathrm{d}^{6}\right.$-DMSO)

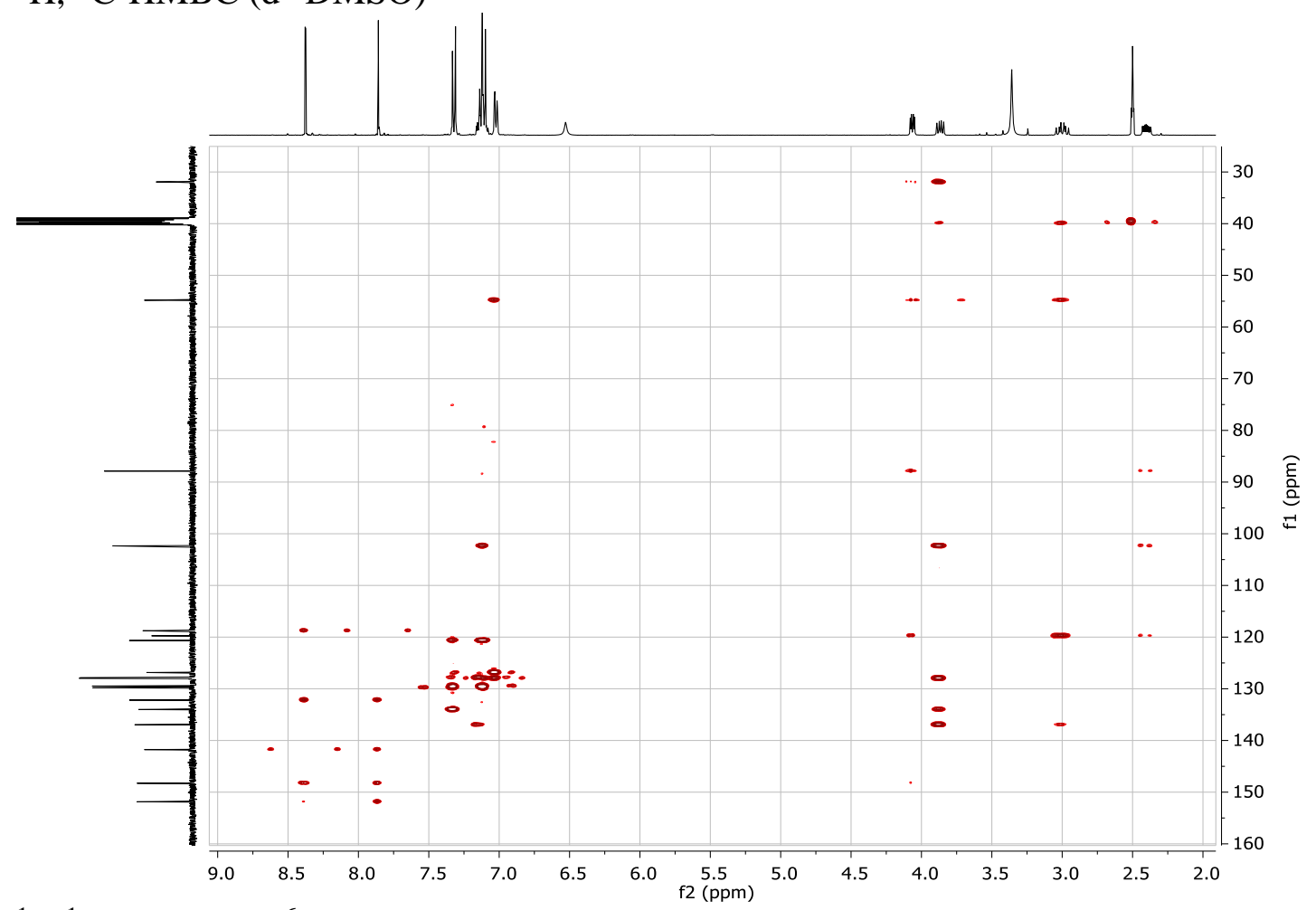

${ }^{1} \mathrm{H},{ }^{1} \mathrm{H}-\mathrm{NOESY}$ (d $\mathrm{d}^{6}$-DMSO)

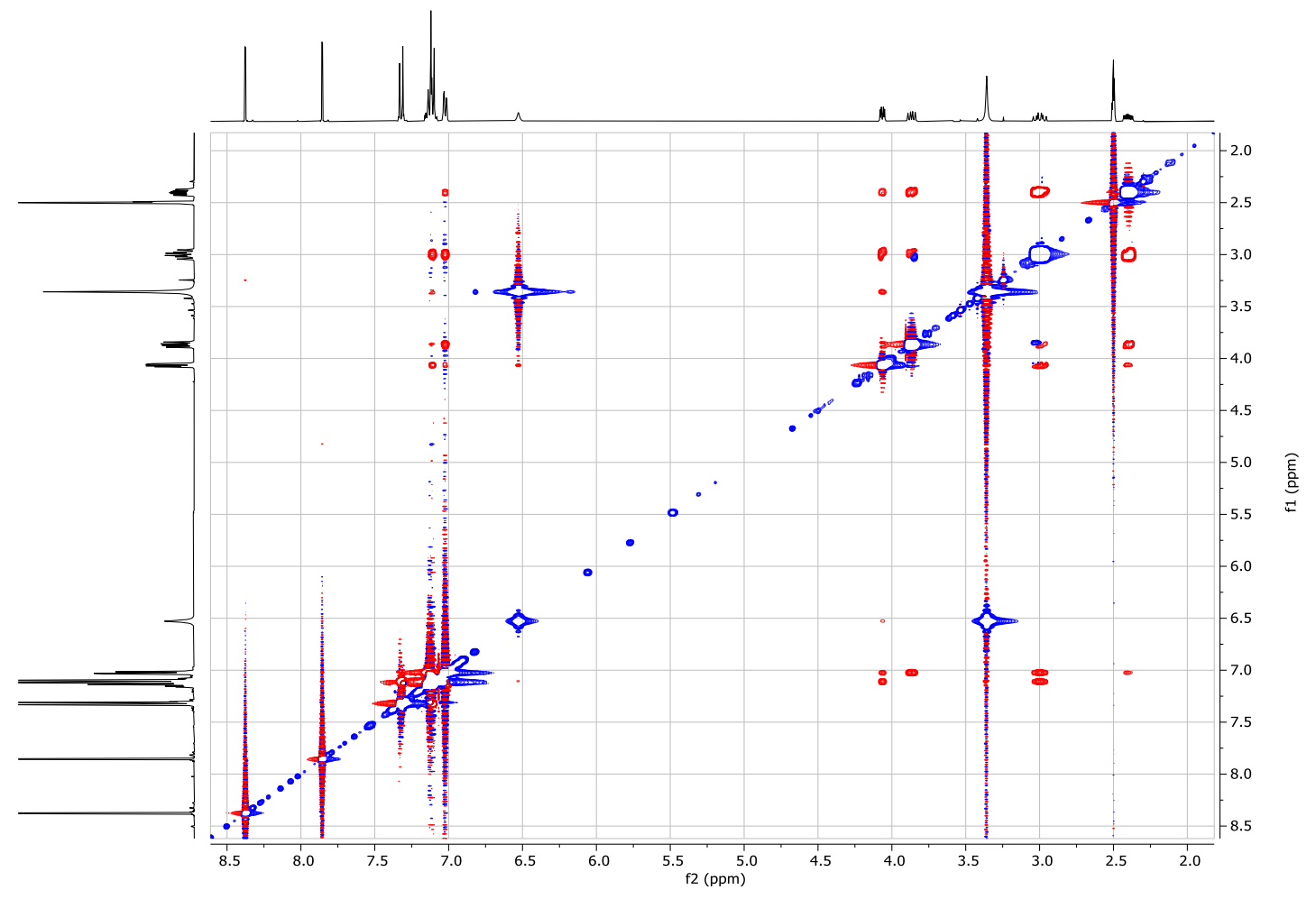


$r a c$-(5aR,6S,8S,8a $R)$-8-(aminomethyl)-5a-(4-bromophenyl)-3-chloro-6-phenyl-5a,6,7,8tetrahydro-8aH-cyclopenta[4,5]furo[3,2-b]pyridin-8a-ol (TFA salt) (16)

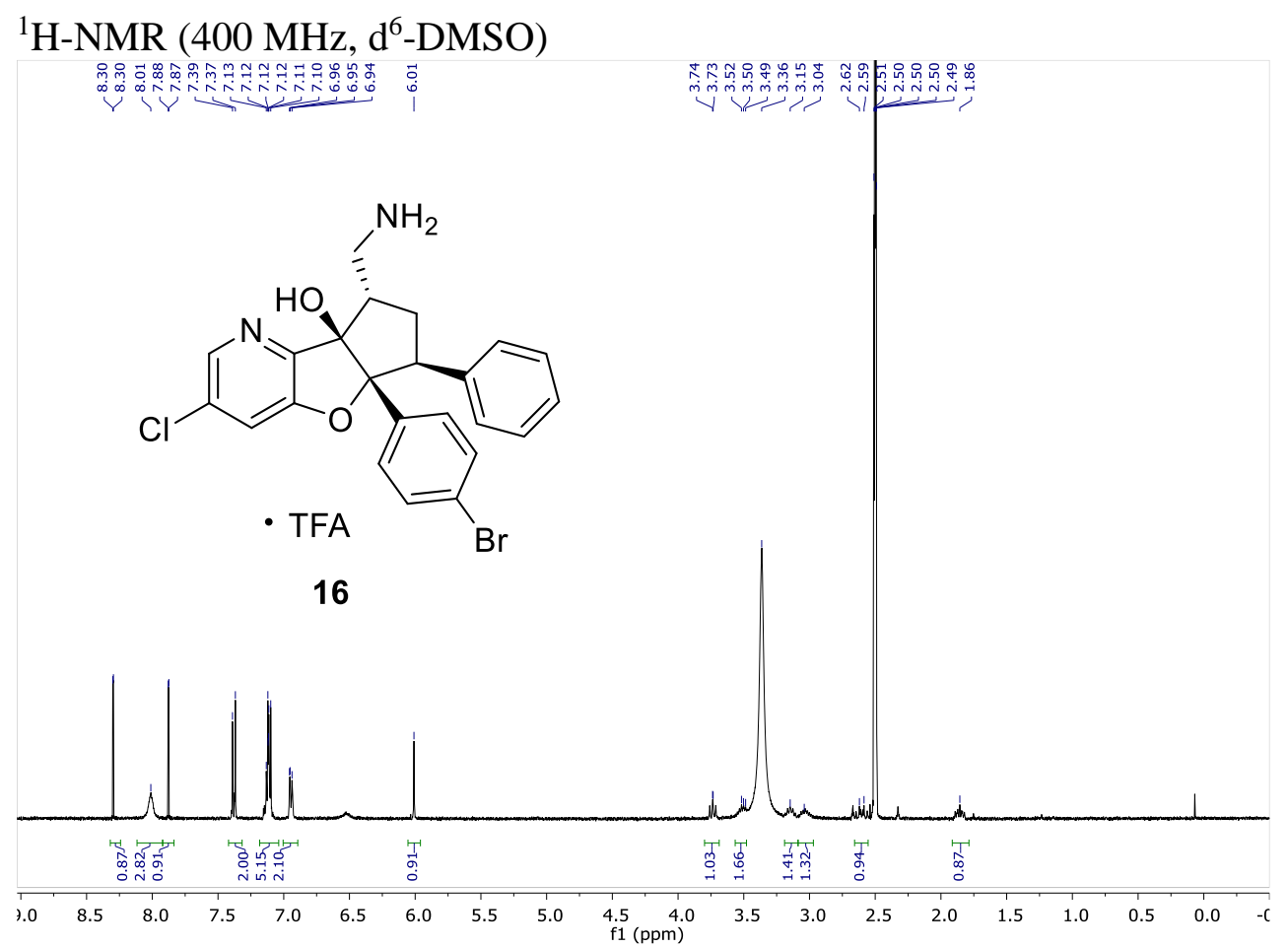

${ }^{13} \mathrm{C}-\mathrm{NMR}\left(101 \mathrm{MHz}, \mathrm{d}^{6}\right.$-DMSO)
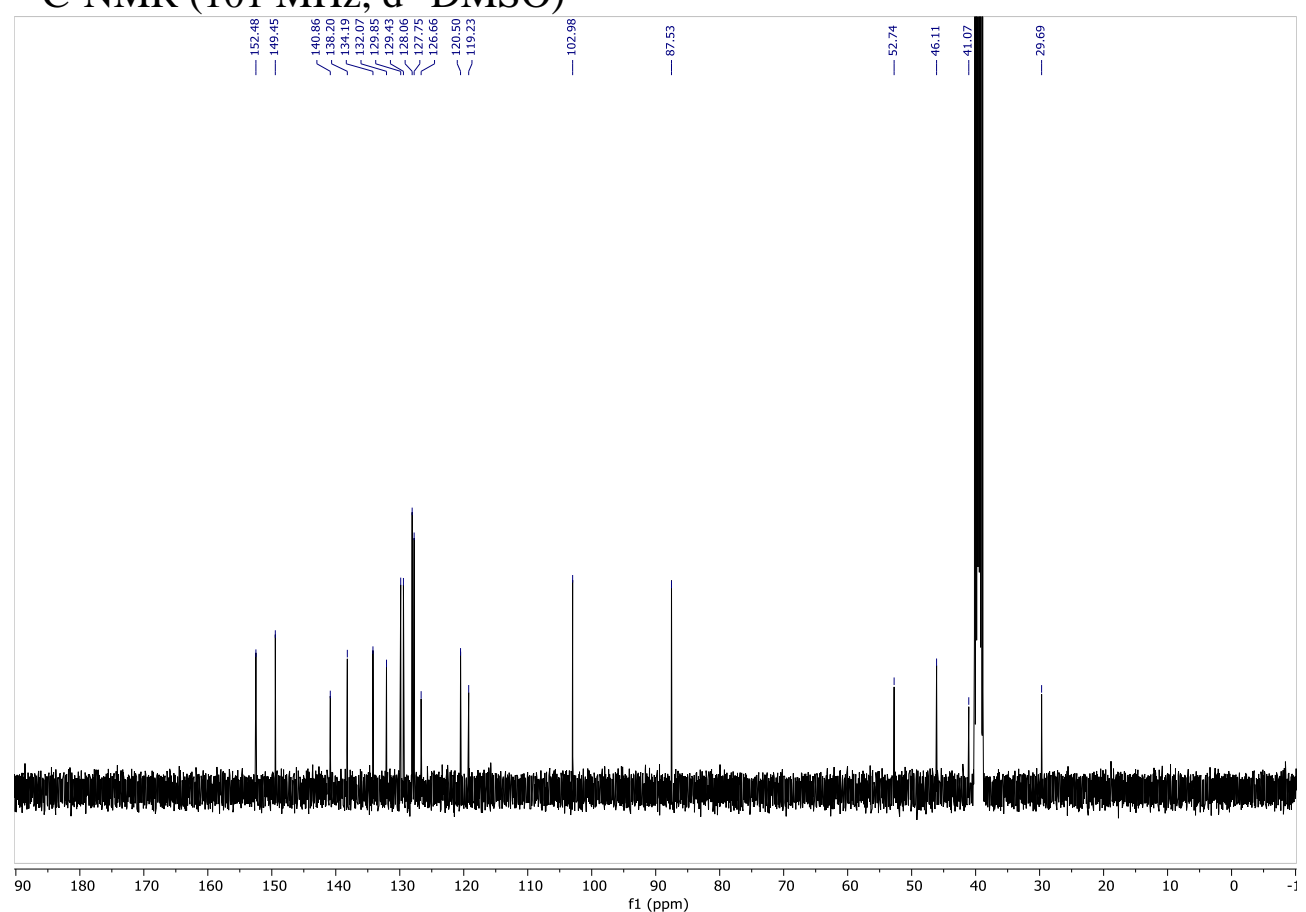
rac-methyl (5aR,6S,7R,8R,8aS)-5a-(4-bromophenyl)-3-chloro-8a-hydroxy-8((methylsulfonyl)-oxy)-6-phenyl-5a,7,8,8a-tetrahydro-6H-cyclopenta[4,5]furo[3,2b]pyridine-7-carboxylate (18)

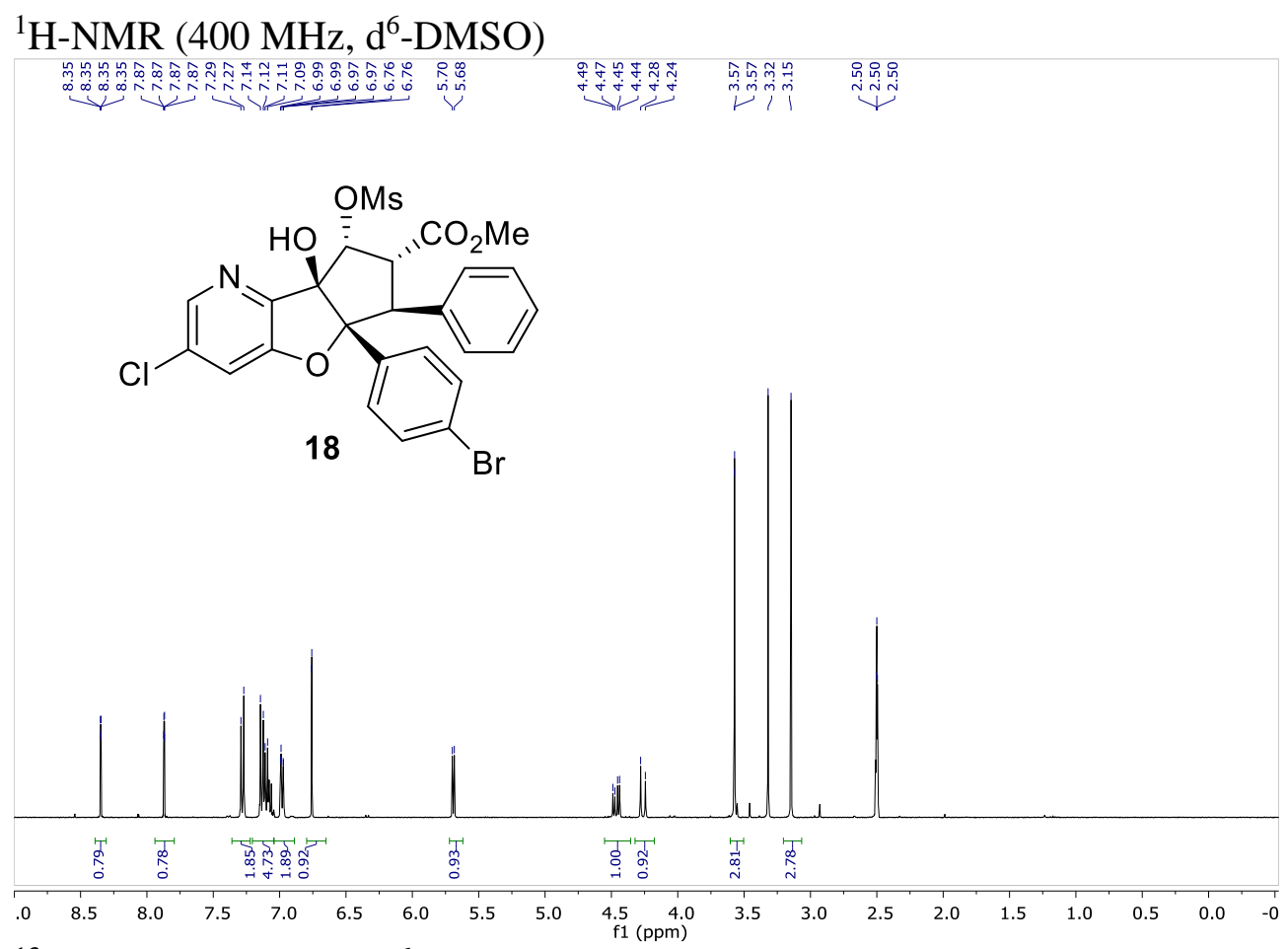

${ }^{13} \mathrm{C}-\mathrm{NMR}$ (101 MHz, d ${ }^{6}$-DMSO)

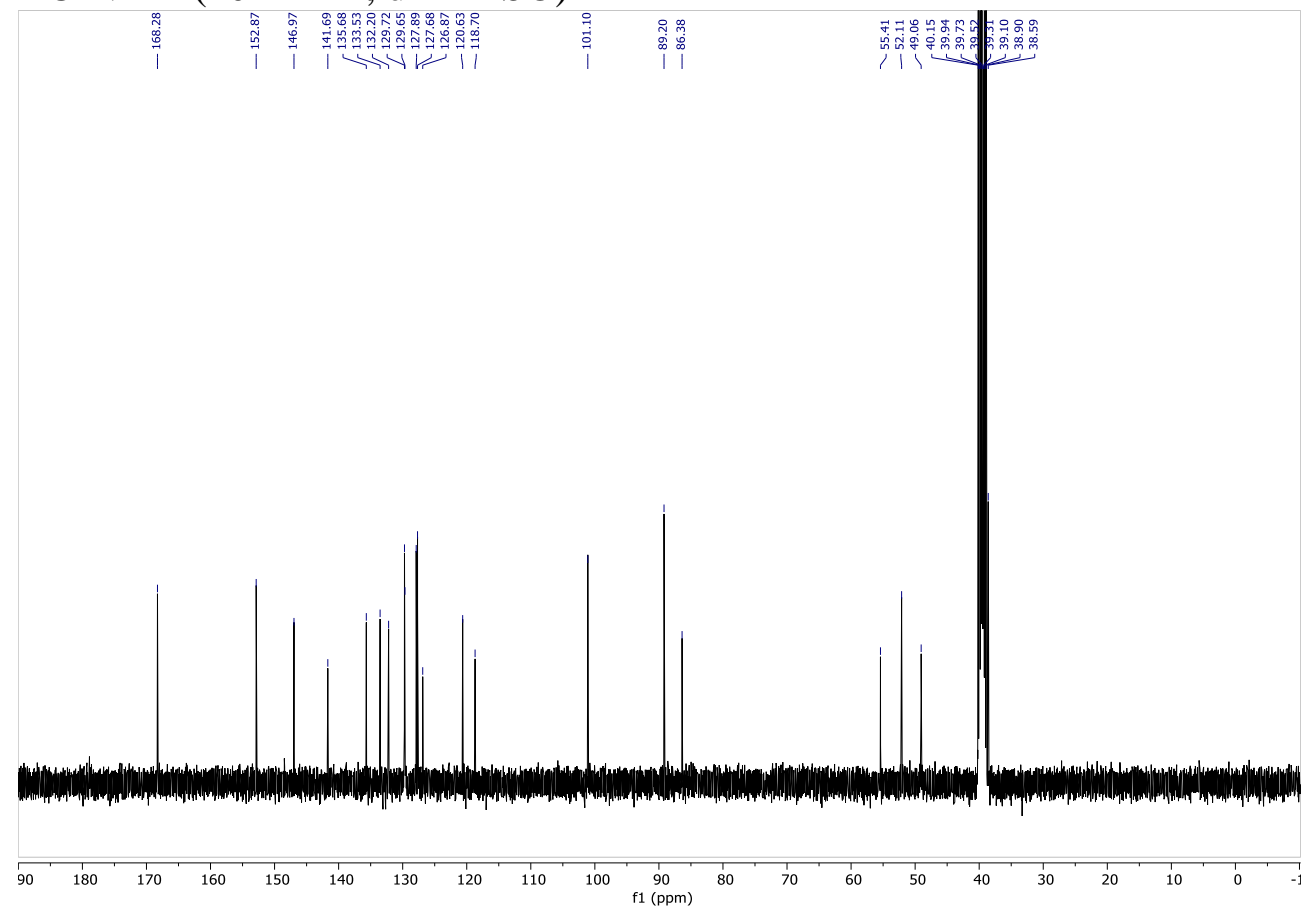


${ }^{1} \mathrm{H},{ }^{1} \mathrm{H}-\mathrm{COSY}\left(\mathrm{d}^{6}\right.$-DMSO)

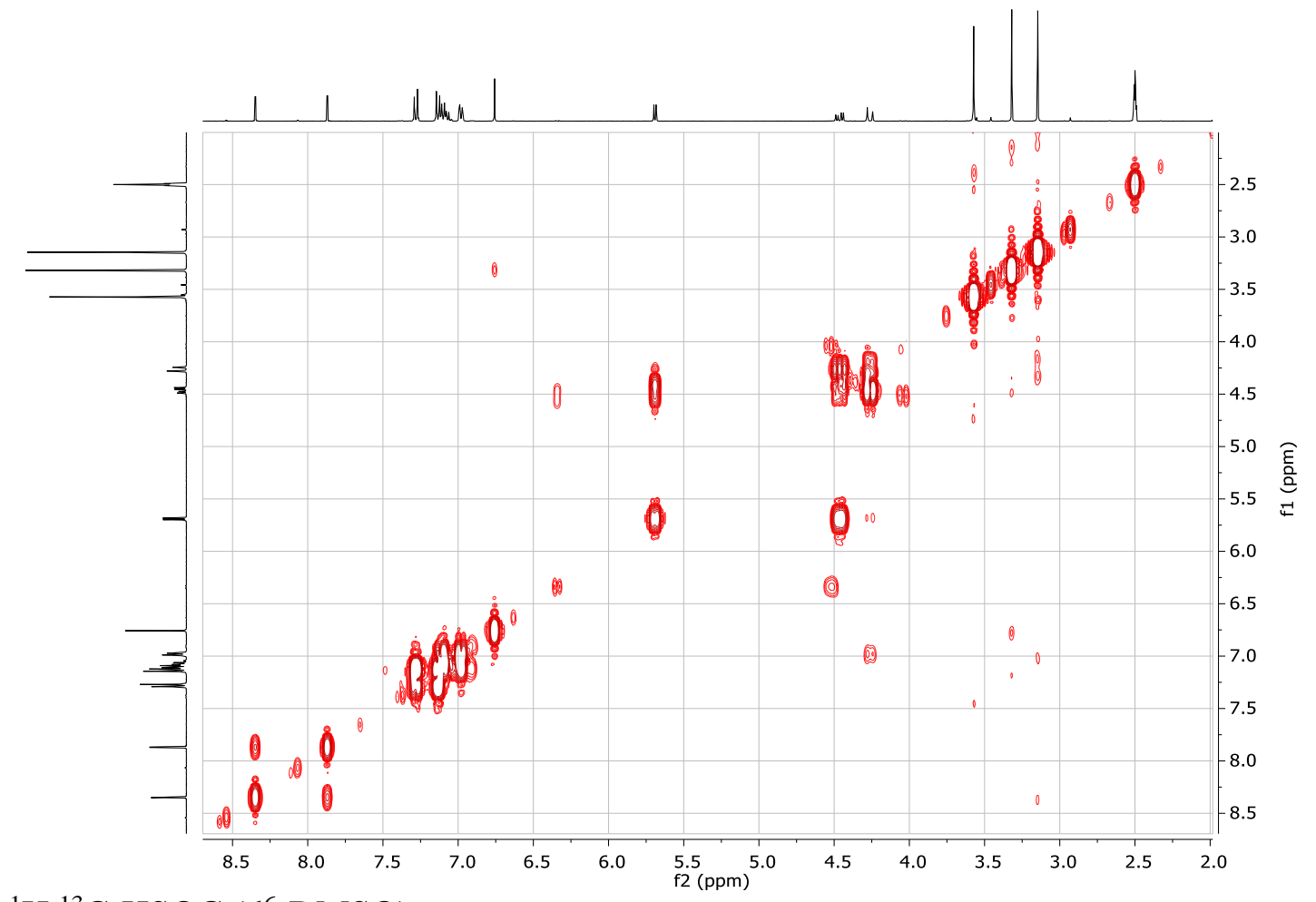

${ }^{1} \mathrm{H},{ }^{13} \mathrm{C}$-HSQC ( $\mathrm{d}^{6}$-DMSO)

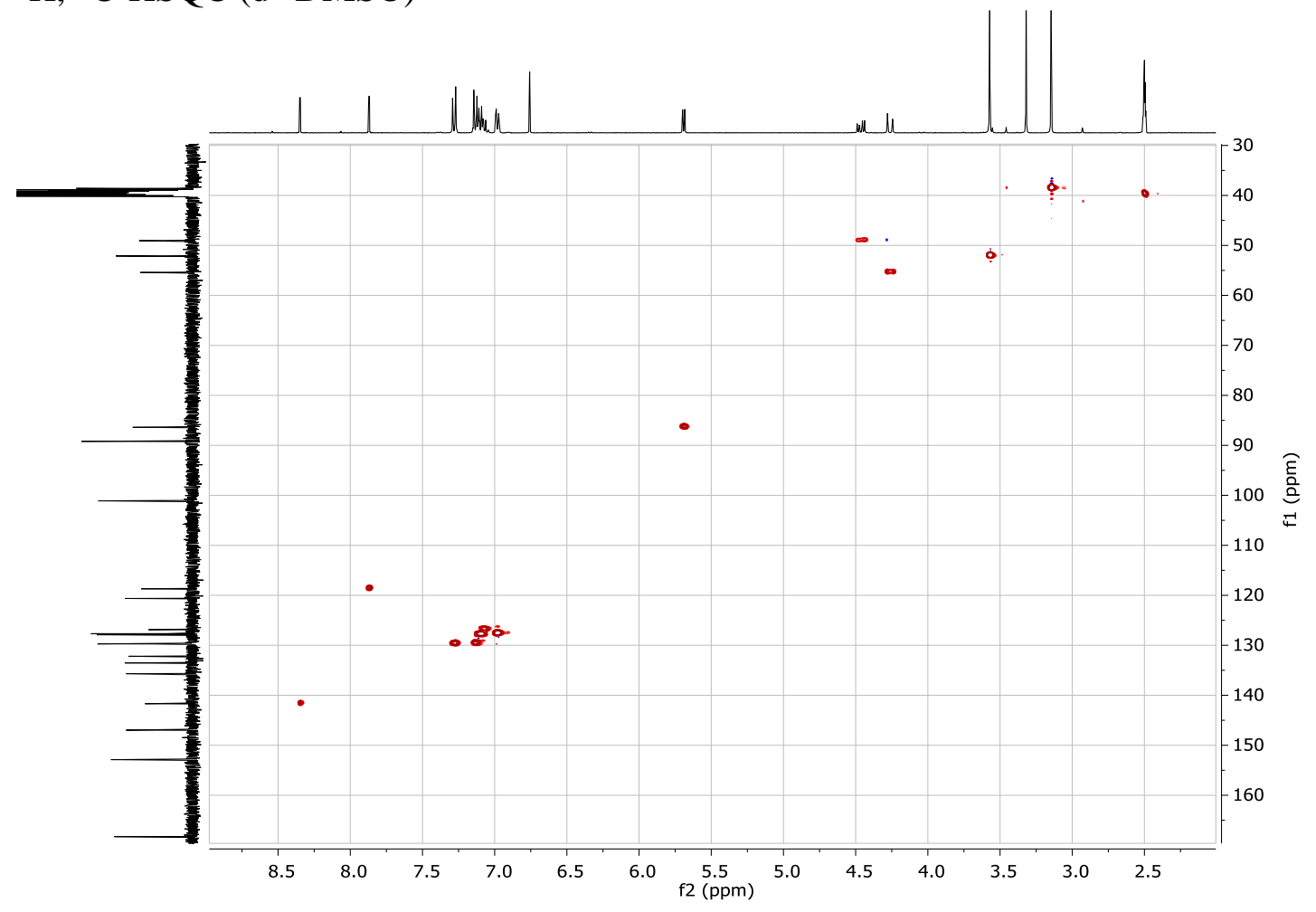


${ }^{1} \mathrm{H},{ }^{1} \mathrm{H}-\mathrm{NOESY}\left(\mathrm{d}^{6}\right.$-DMSO)

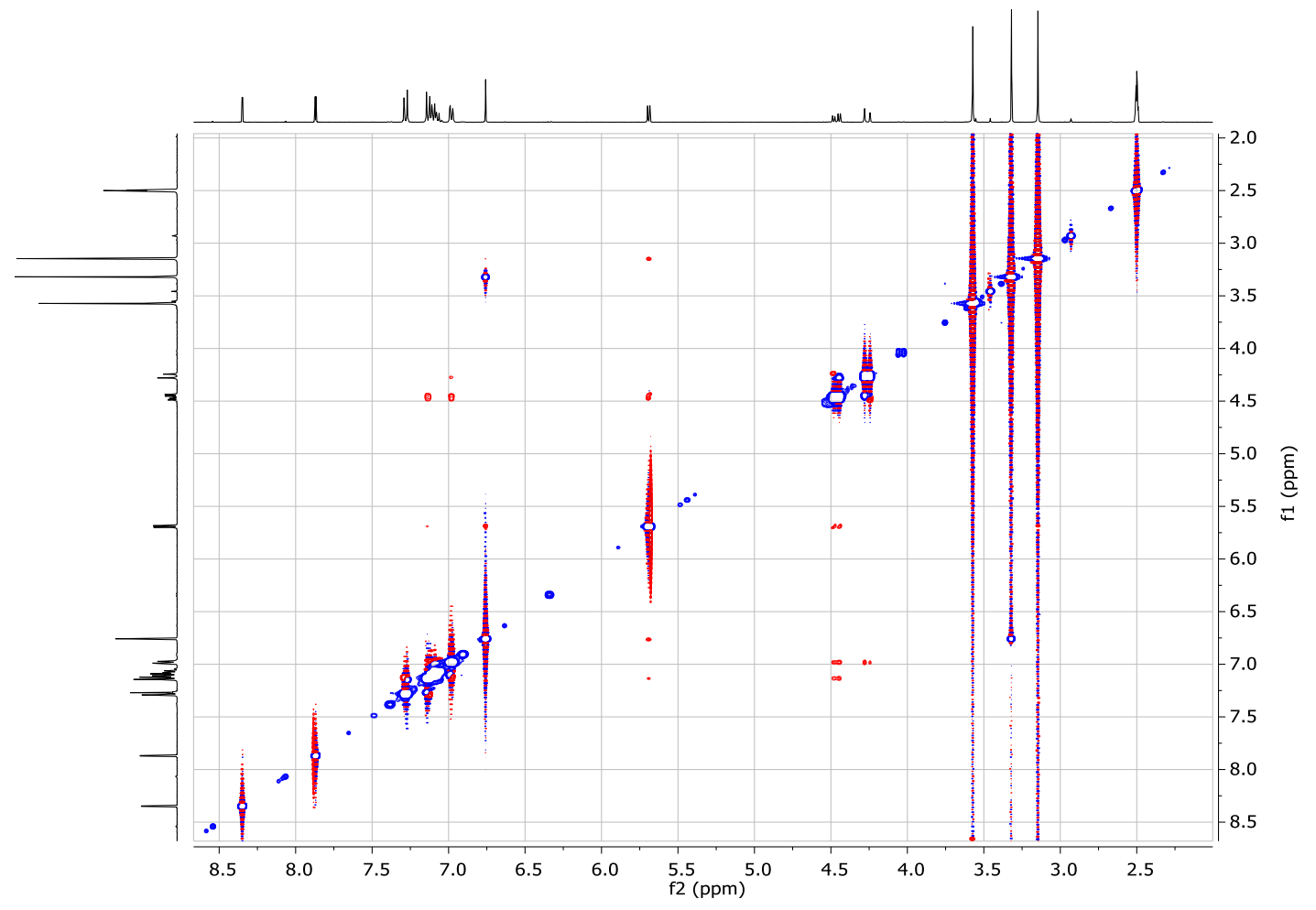


rac-methyl

(5a $R, 6 S, 7 R, 8 R, 8 \mathrm{a} R)-5 \mathrm{a}-(4-b r o m o p h e n y l)-3-c h l o r o-8-c y a n o-8 a-h y d r o x y-6-$ phenyl-5a,7,8,8a-tetrahydro-6H-cyclopenta[4,5]furo[3,2-b]pyridine-7-carboxylate (21)

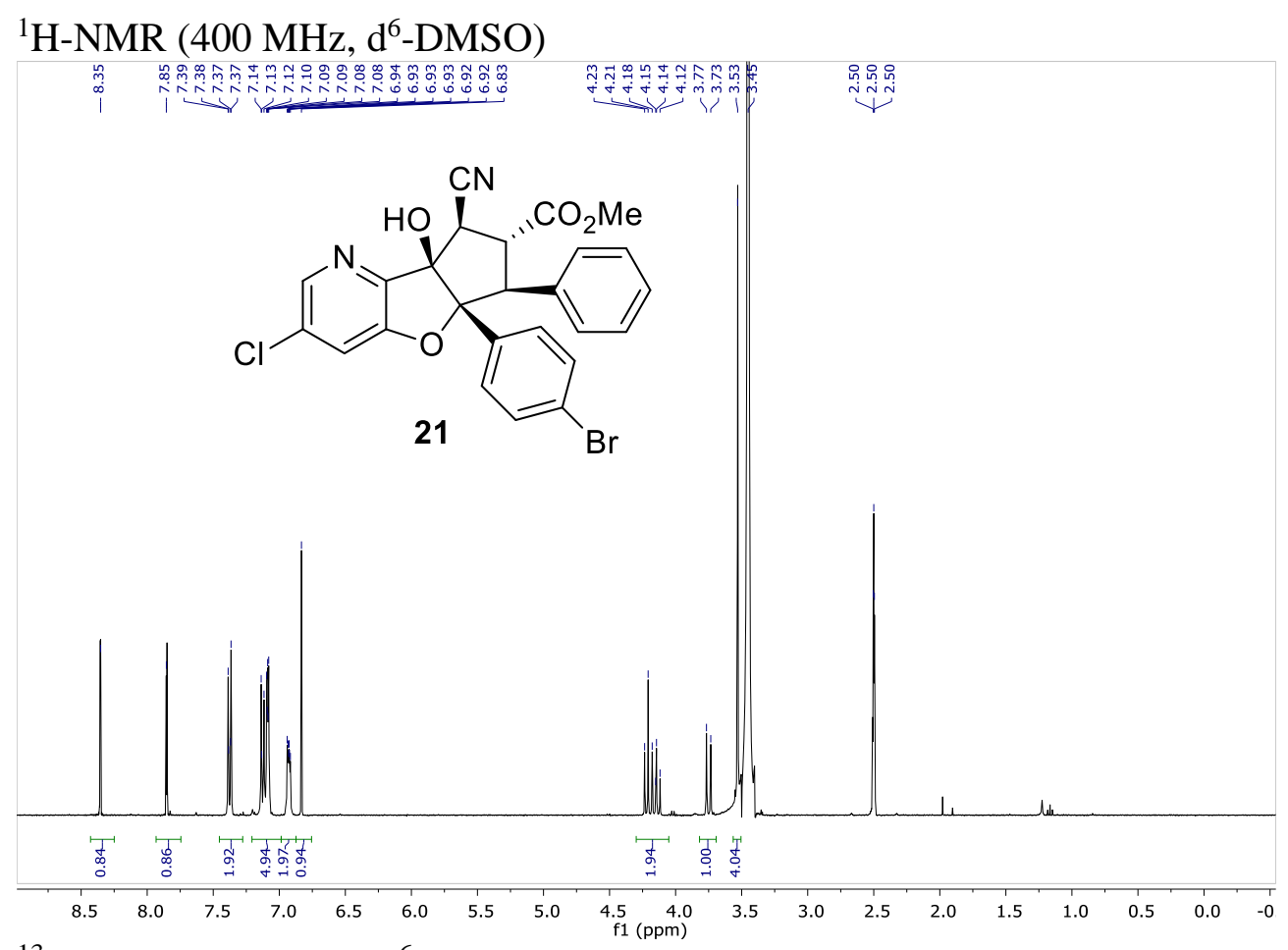

${ }^{13} \mathrm{C}-\mathrm{NMR}\left(101 \mathrm{MHz}, \mathrm{d}^{6}\right.$-DMSO)

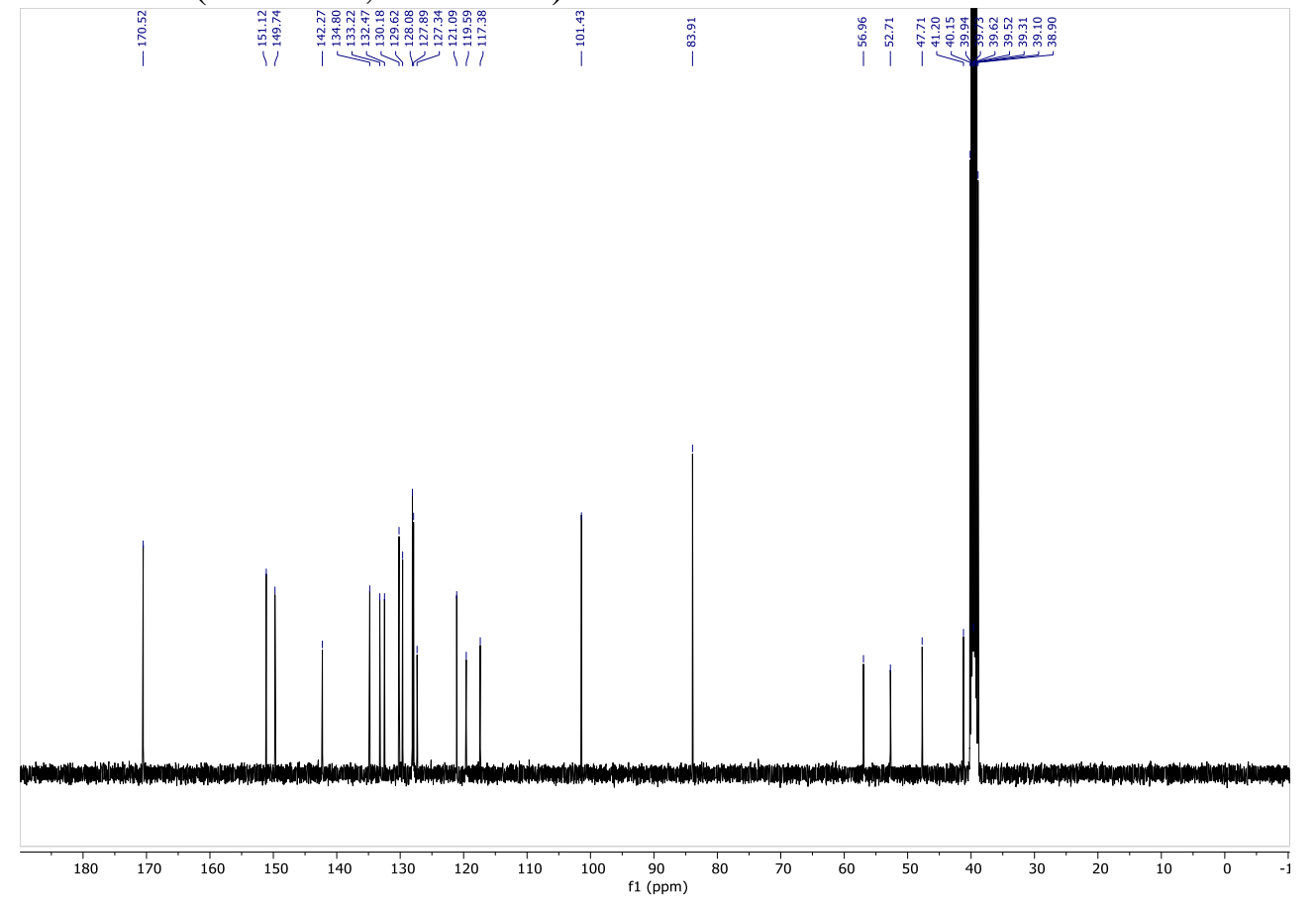


${ }^{1} \mathrm{H},{ }^{1} \mathrm{H}-\mathrm{COSY}\left(\mathrm{d}^{6}\right.$-DMSO)

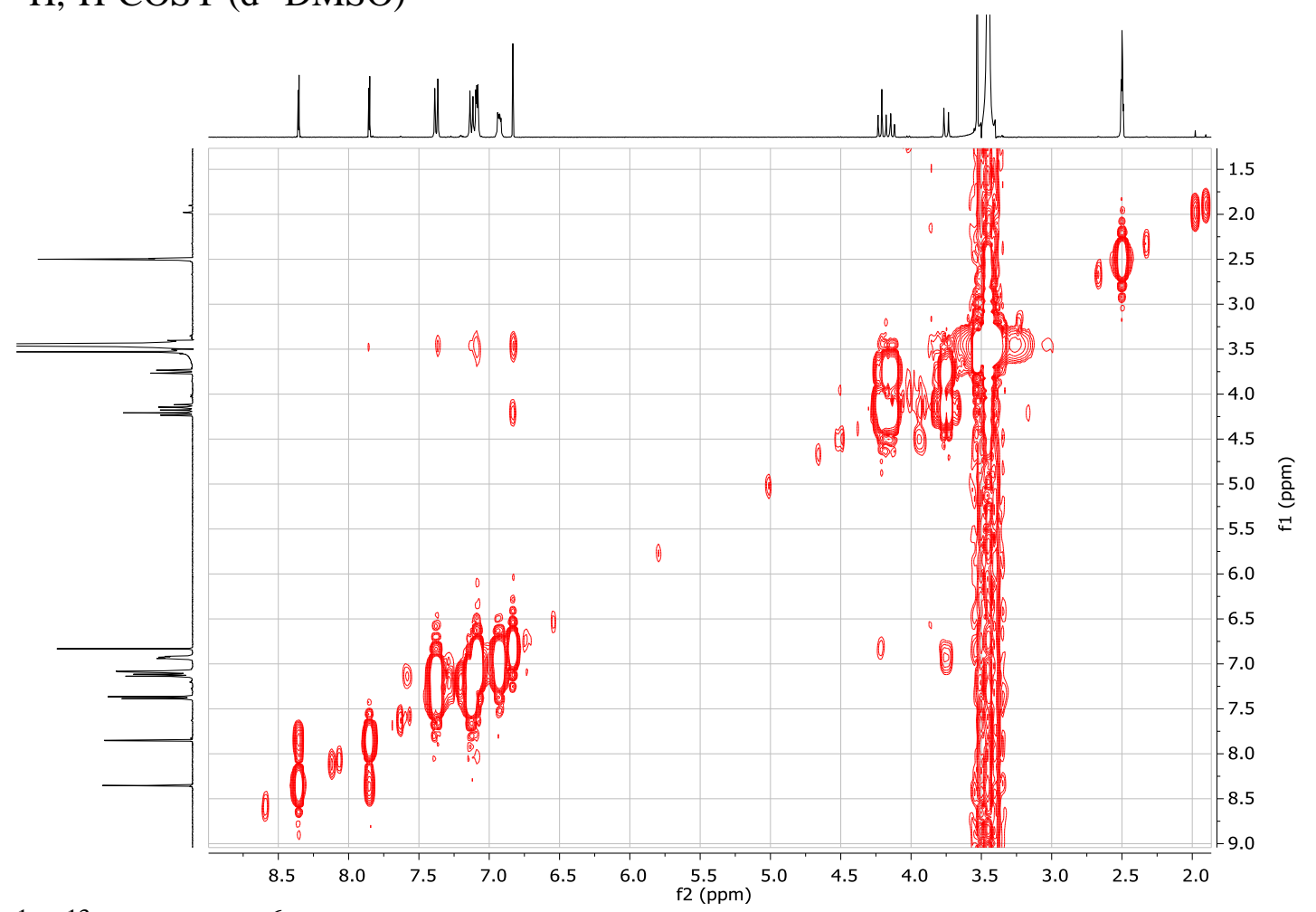

${ }^{1} \mathrm{H},{ }^{13} \mathrm{C}$-HSQC ( $\mathrm{d}^{6}$-DMSO)

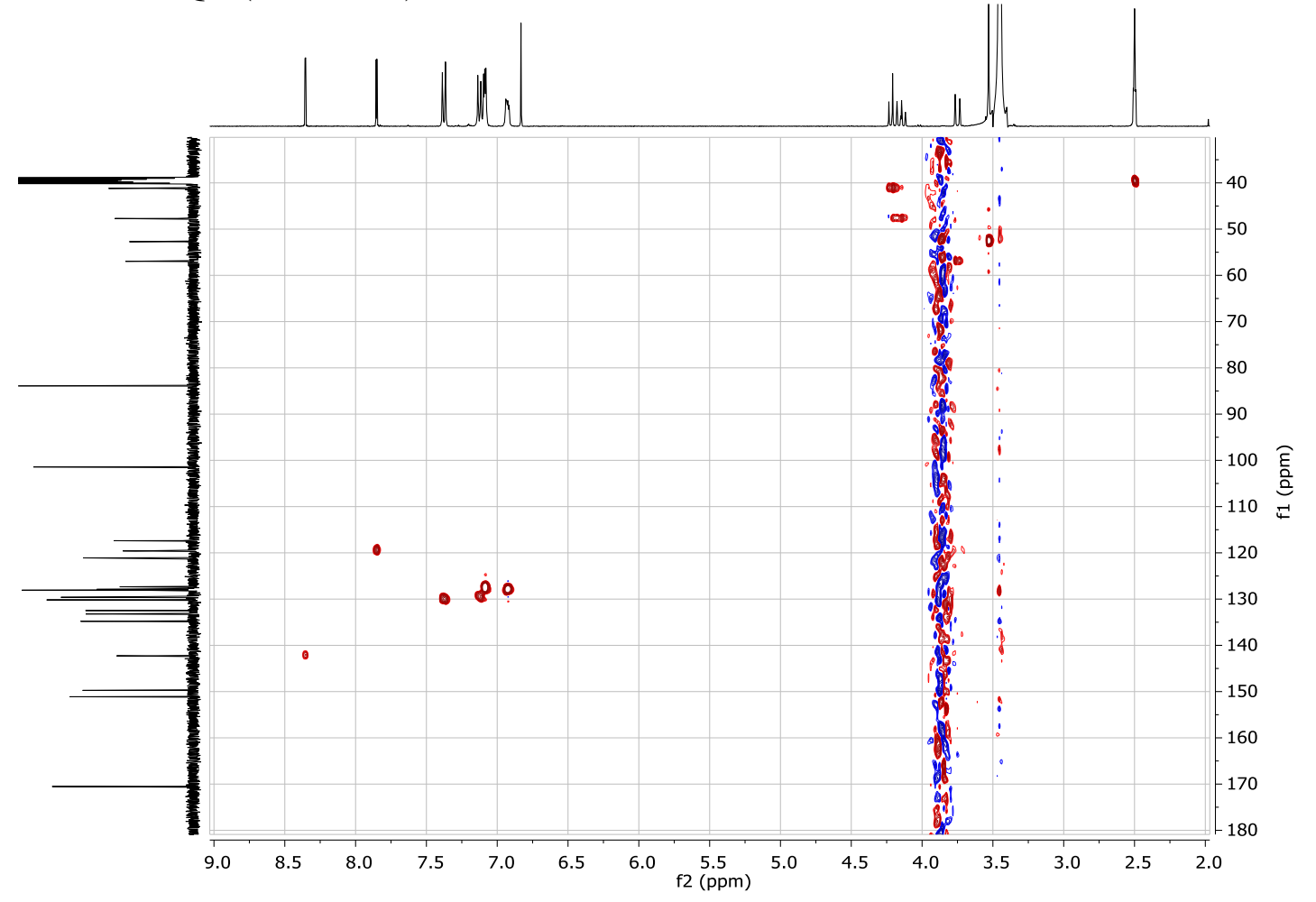




\section{${ }^{1} \mathrm{H},{ }^{1} \mathrm{H}-\mathrm{NOESY}\left(\mathrm{d}^{6}\right.$-DMSO)}

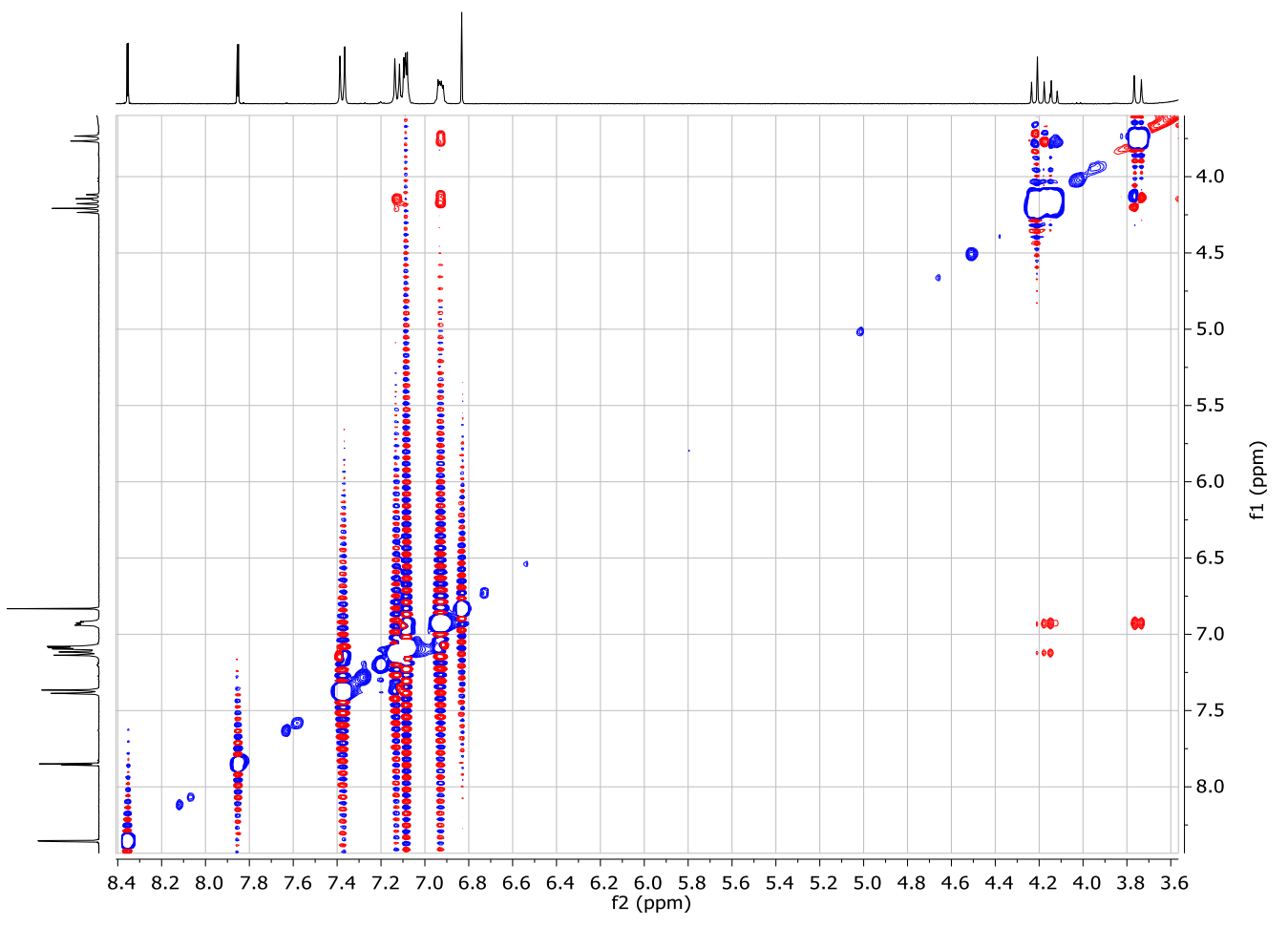




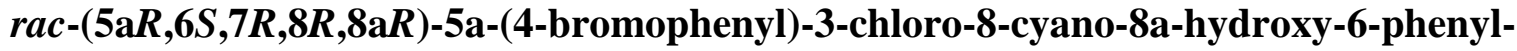
5a,7,8,8a-tetrahydro-6H-cyclopenta[4,5]furo[3,2-b]pyridine-7-carboxylic acid (22)
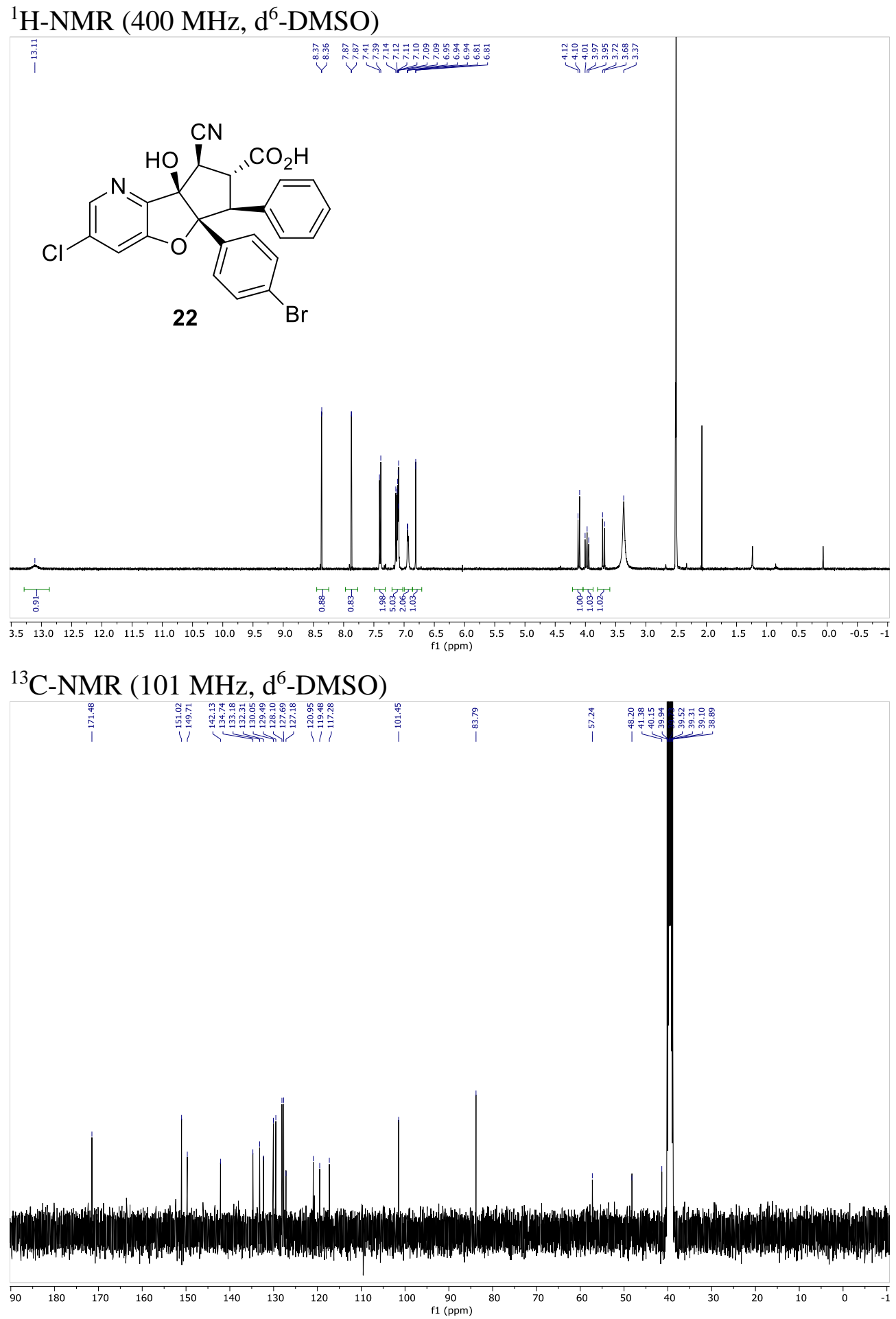
$r a c$-(5a $R, 6 S, 8 R, 8 \mathrm{a} R)$-5a-(4-bromophenyl)-3-chloro-8a-hydroxy-6-phenyl-5a,7,8,8atetrahydro-6H-cyclopenta[4,5]furo[3,2-b]pyridine-8-carbonitrile (23)

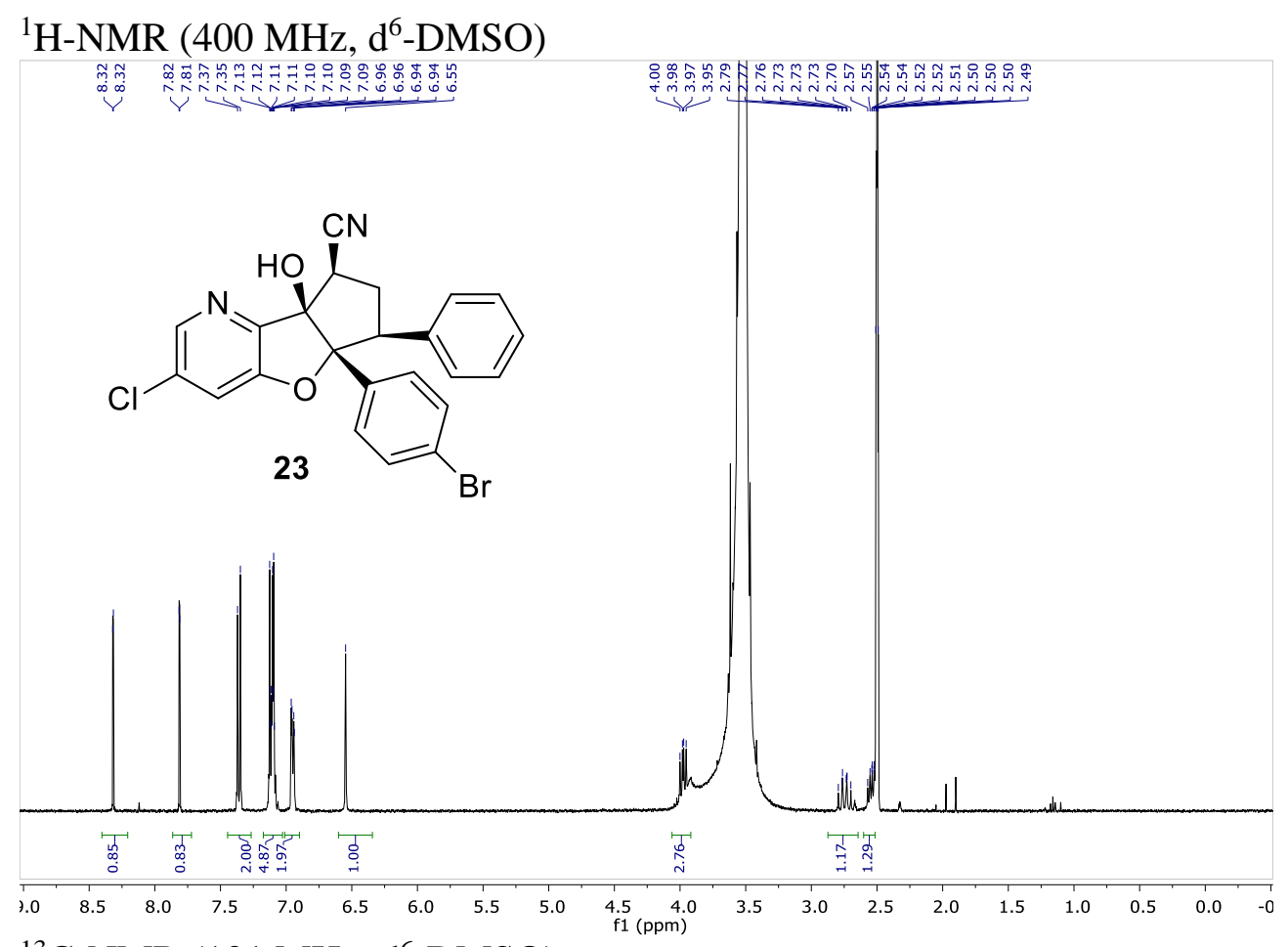

${ }^{13} \mathrm{C}-\mathrm{NMR}\left(101 \mathrm{MHz}, \mathrm{d}^{6}\right.$-DMSO)
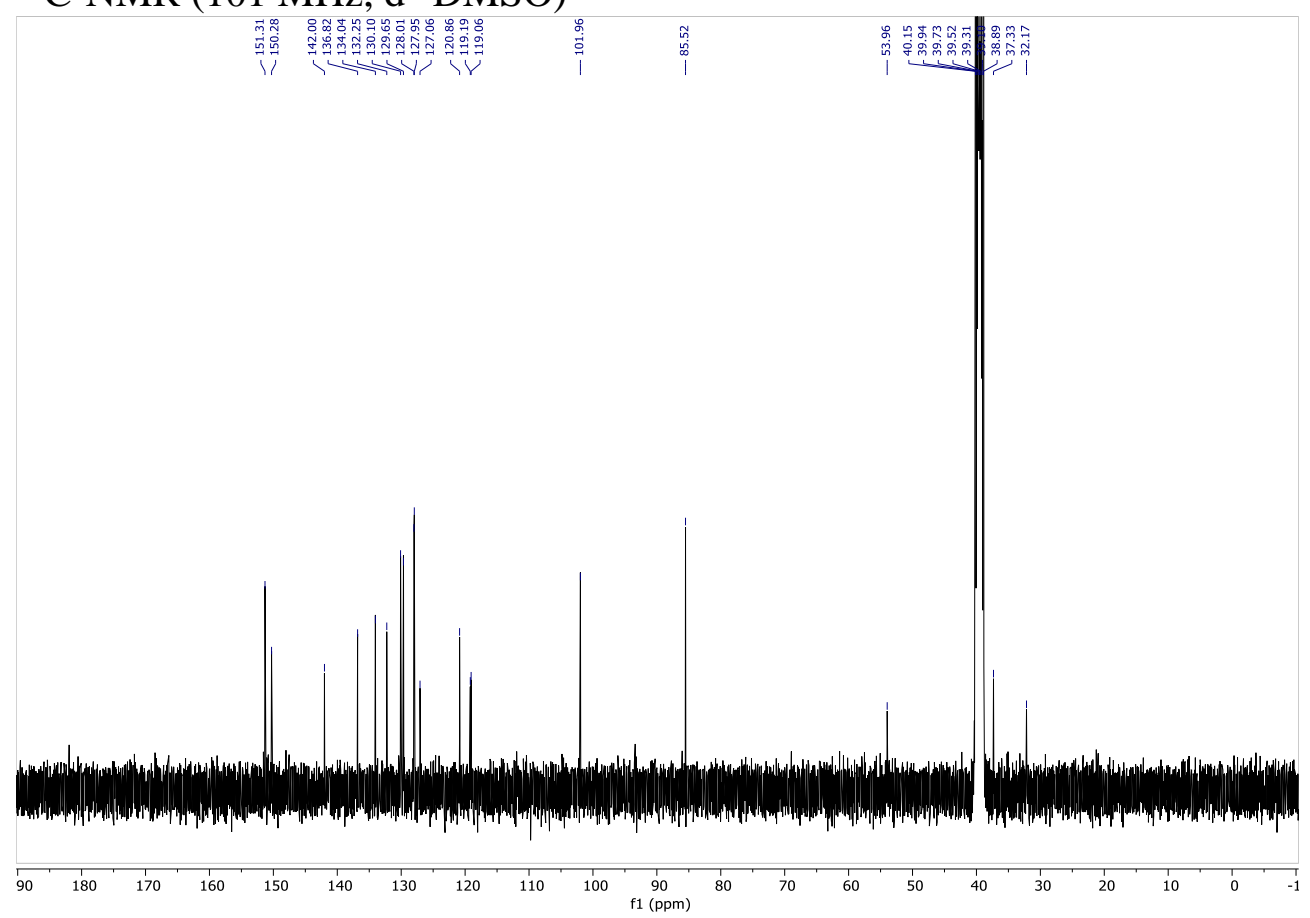
$r a c$-(5aR,6S,8R,8aR)-8-(aminomethyl)-5a-(4-bromophenyl)-3-chloro-6-phenyl-5a,6,7,8tetrahydro-8aH-cyclopenta[4,5]furo[3,2-b]pyridin-8a-ol (TFA salt) (24)

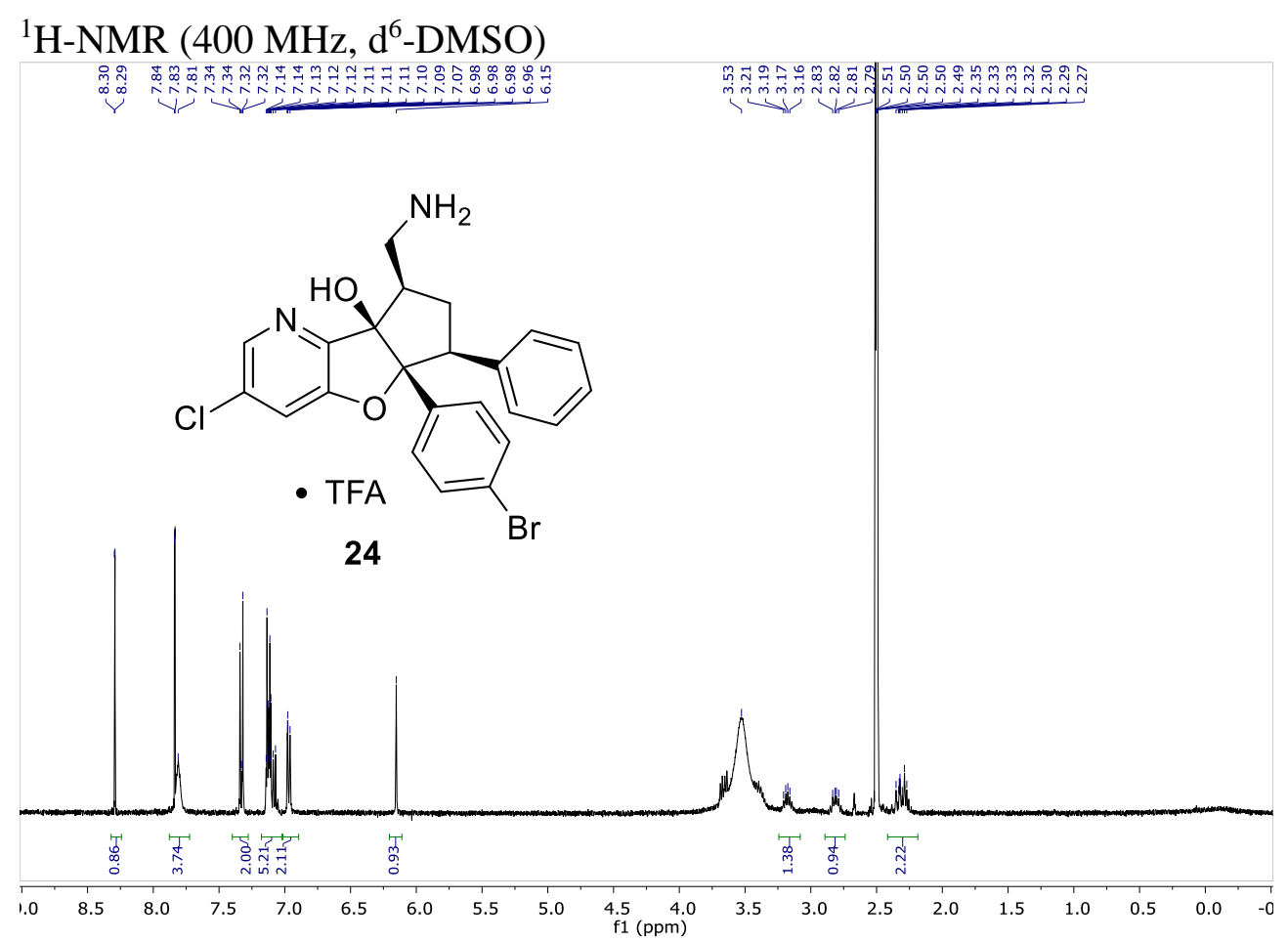

${ }^{13} \mathrm{C}-\mathrm{NMR}\left(101 \mathrm{MHz}, \mathrm{d}^{6}\right.$-DMSO)

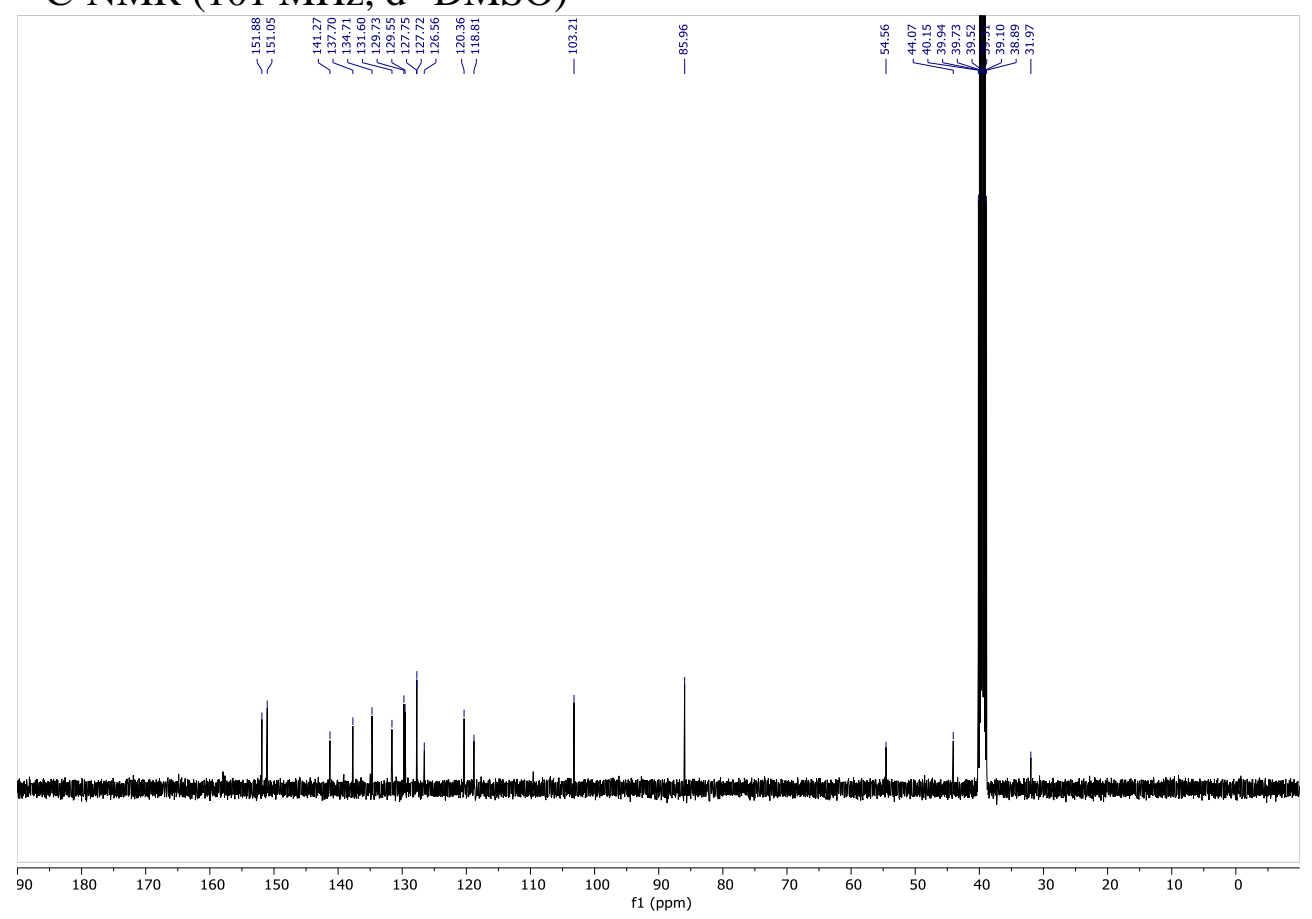


rac-4-((5aR,6S,8R,8aR)-8-(aminomethyl)-3-chloro-8a-hydroxy-6-phenyl-6,7,8,8atetrahydro-5aH-cyclopenta[4,5]furo[3,2-b]pyridin-5a-yl)benzonitrile (TFA salt) (25)

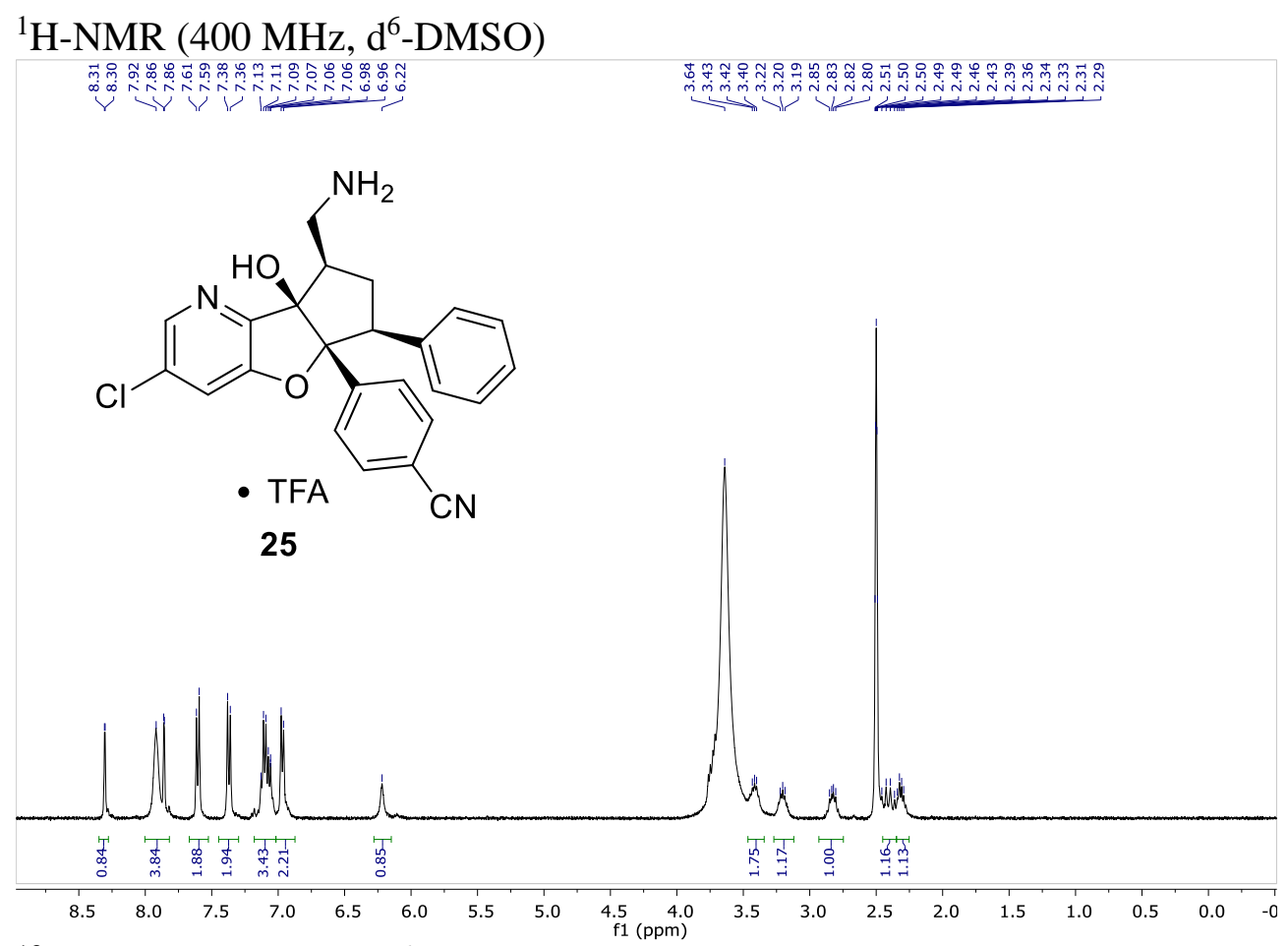

${ }^{13} \mathrm{C}-\mathrm{NMR}\left(126 \mathrm{MHz}, \mathrm{d}^{6}\right.$-DMSO)
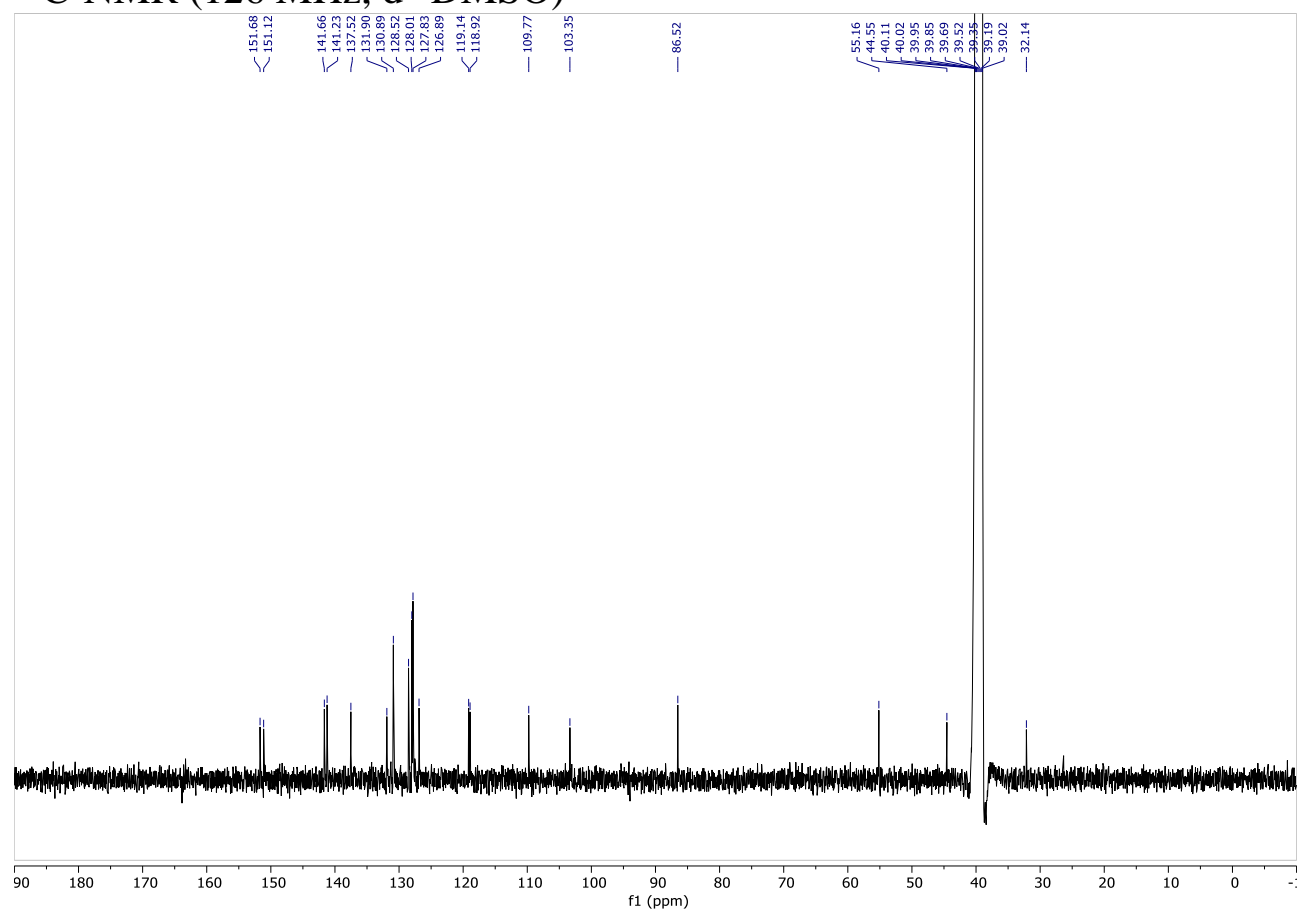
rac-(1aS,3S,3aR,8bS)-3a-(4-bromophenyl)-6-chloro-3-phenyl-1a,2,3,3atetrahydrooxireno[ $\left.2^{\prime \prime}, 3^{\prime \prime}: 1^{\prime}, 5^{\prime}\right]$ cyclopenta[1',2':4,5]furo[3,2-b]pyridine (17)

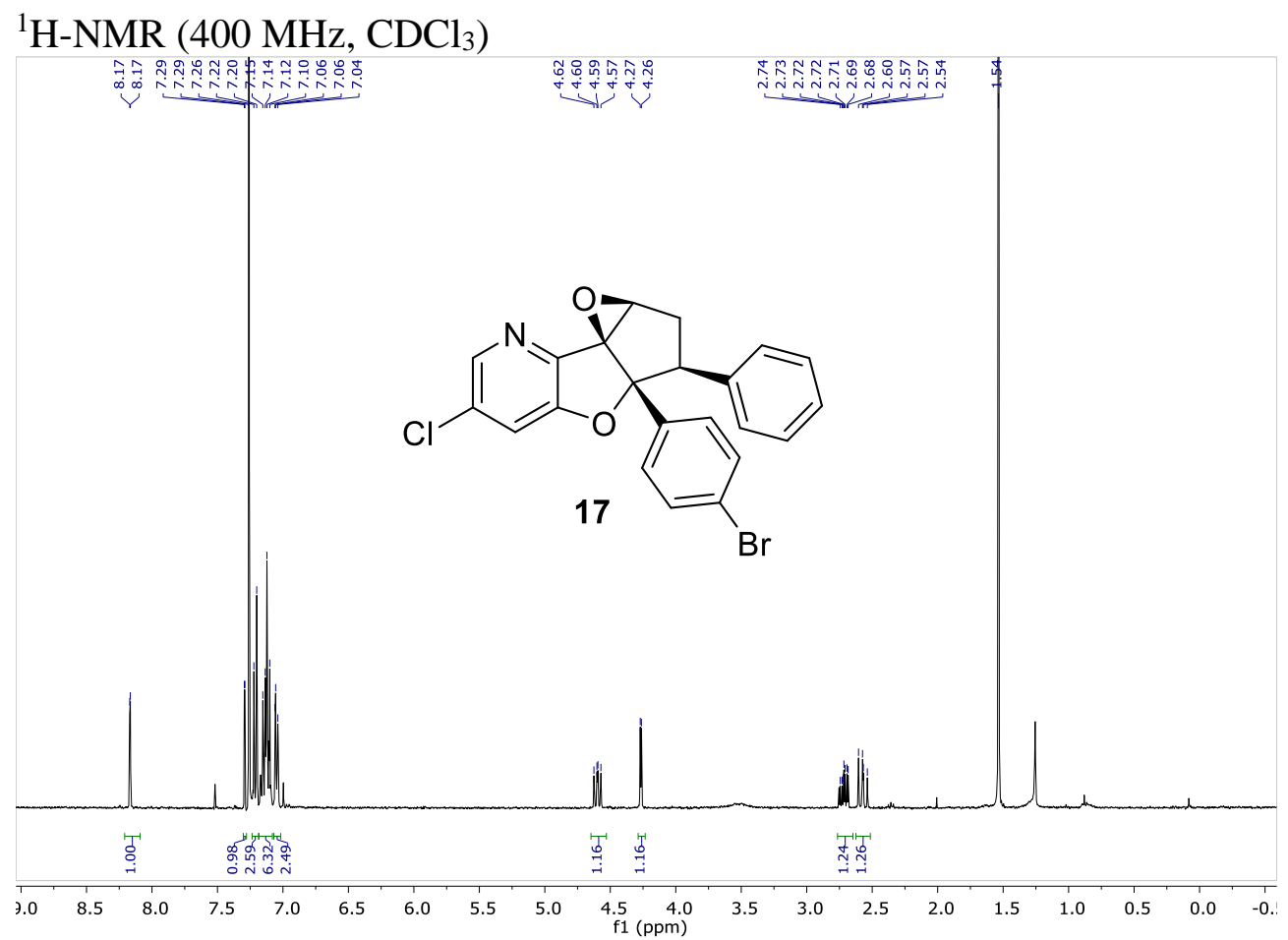

${ }^{13} \mathrm{C}-\mathrm{NMR}\left(101 \mathrm{MHz}, \mathrm{CDCl}_{3}\right)$

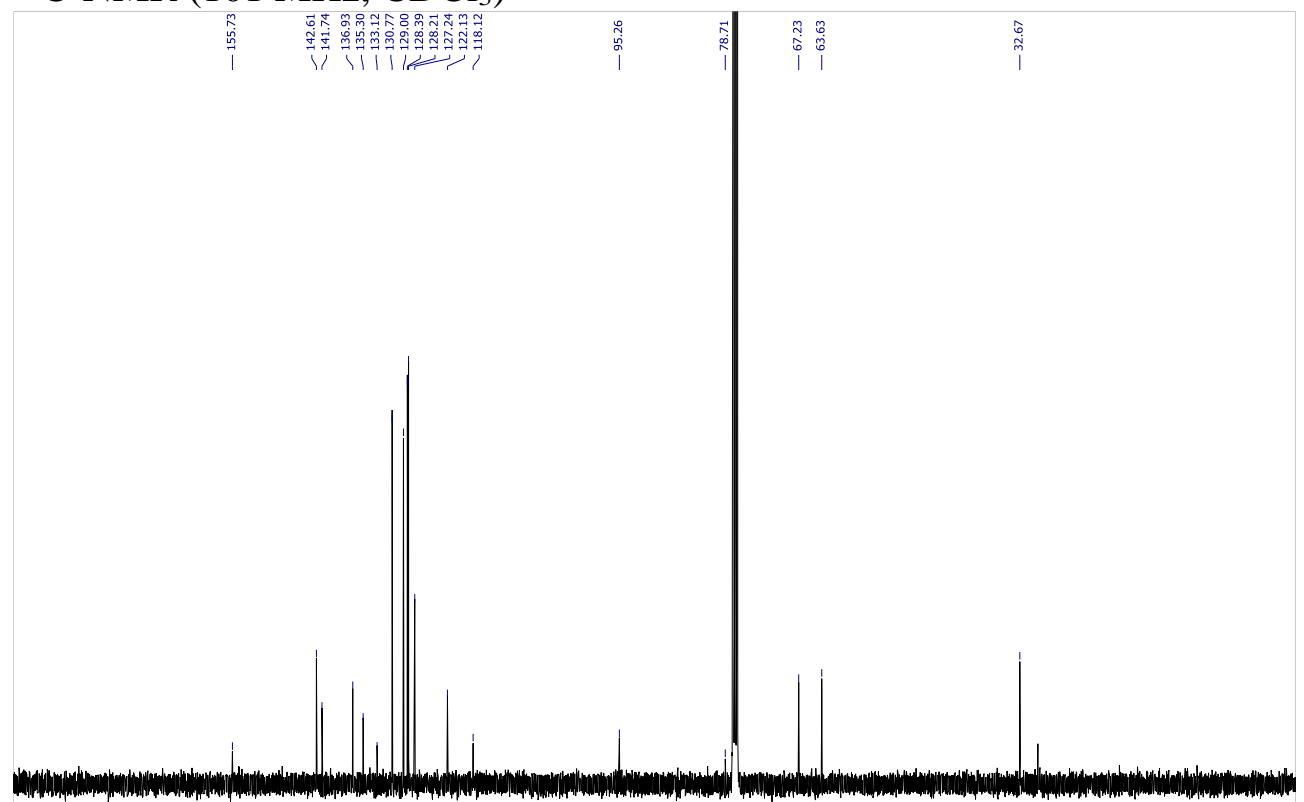

$\begin{array}{llllllllllllllllllll}180 & 170 & 160 & 150 & 140 & 130 & 120 & 110 & 100 & 90 & 90 & 70 & 60 & 50 & 40 & 30 & 20 & 10 & 0 & \mid / 1\end{array}$ 
rac-methyl $\quad(1 \mathrm{a} S, 2 R, 3 S, 3 \mathrm{a} R, 8 \mathrm{~b} S)$-3a-(4-bromophenyl)-6-chloro-3-phenyl-1a,2,3,3a-tetrahydro-oxireno[2",3": $\left.1^{\prime}, 5^{\prime}\right]$ cyclopenta[1',2':4,5]furo[3,2-b]pyridine-2-carboxylate (19)

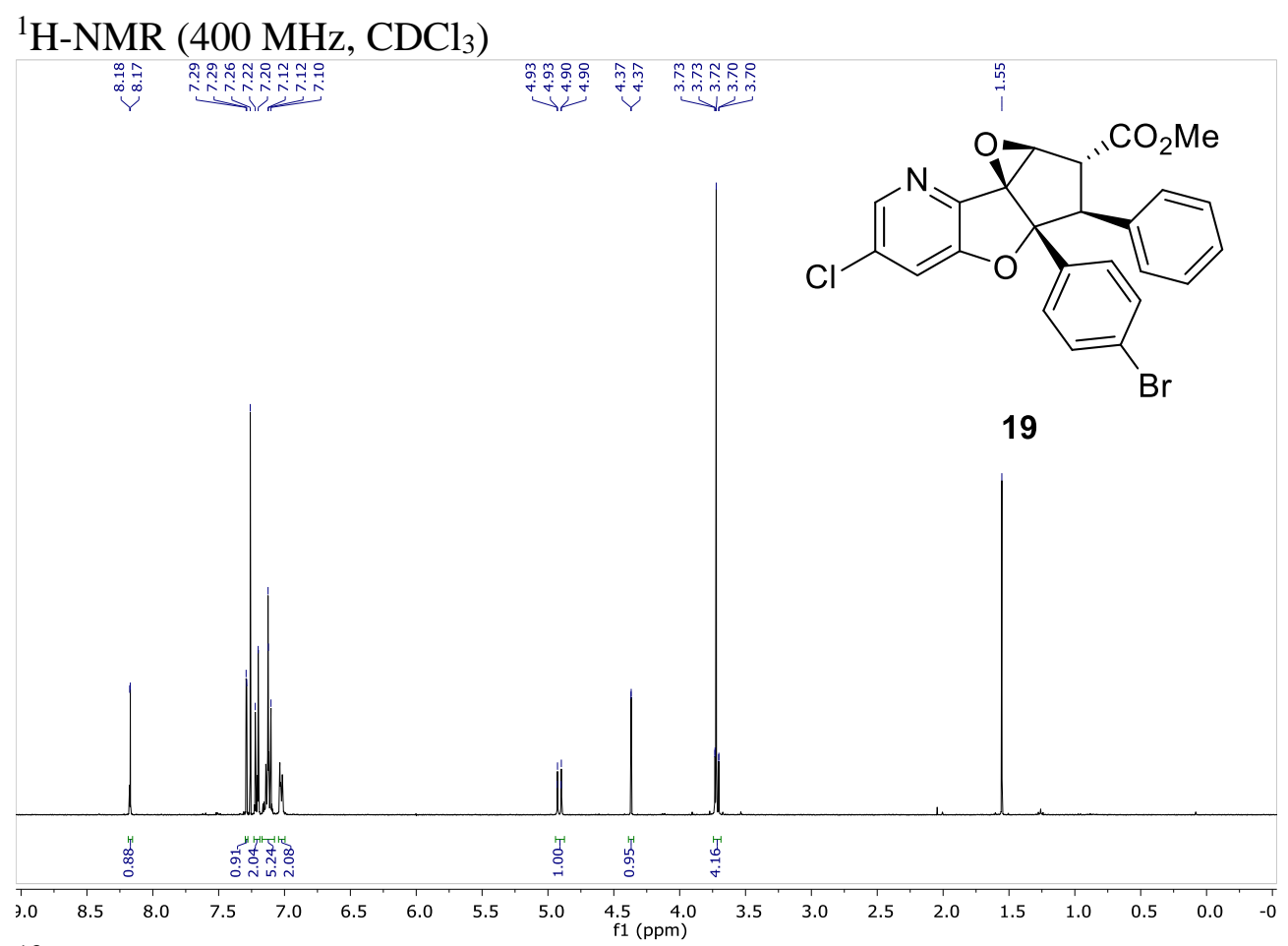

${ }^{13} \mathrm{C}-\mathrm{NMR}\left(101 \mathrm{MHz}, \mathrm{CDCl}_{3}\right)$
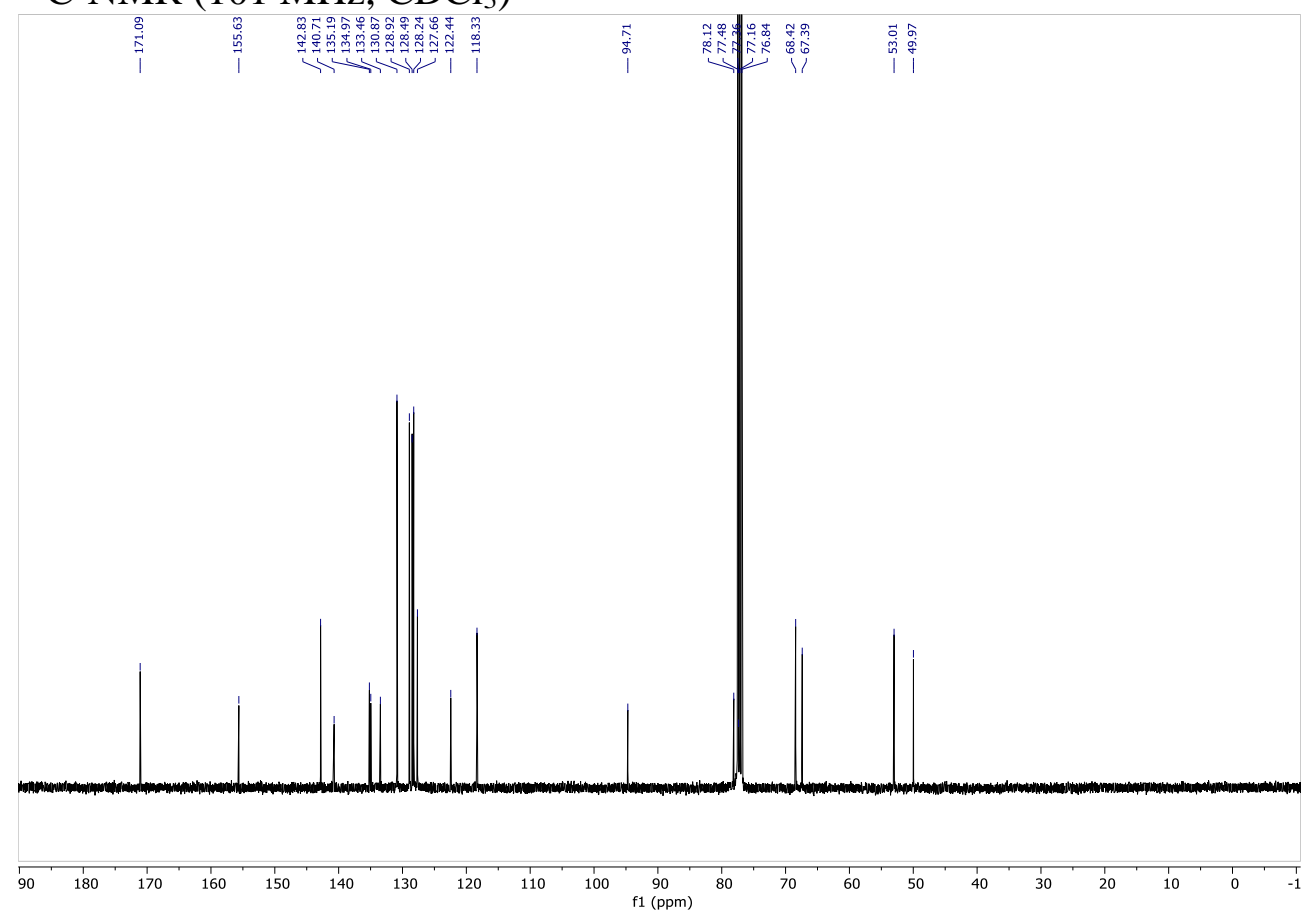
rac-(5aR,6S,7R,8R,8aS)-5a-(4-bromophenyl)-3-chloro-7-(methoxycarbonyl)-6-phenyl5a,6,7,8-tetrahydro-8aH-cyclopenta[4,5]furo[3,2-b]pyridine-8,8a-diyl dibenzoate (S2)

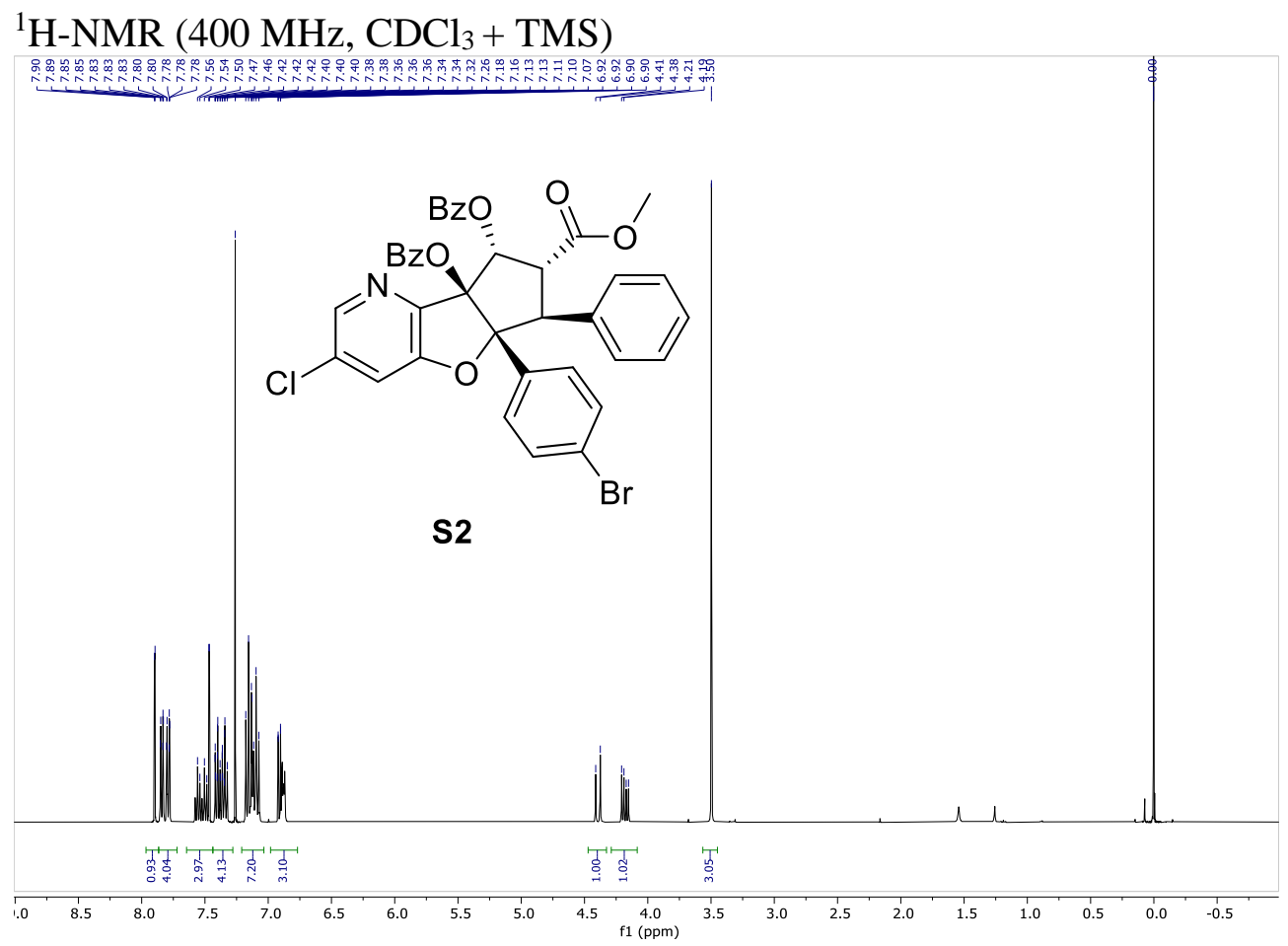

${ }^{13} \mathrm{C}-\mathrm{NMR}\left(101 \mathrm{MHz}, \mathrm{CDCl}_{3}+\mathrm{TMS}\right)$

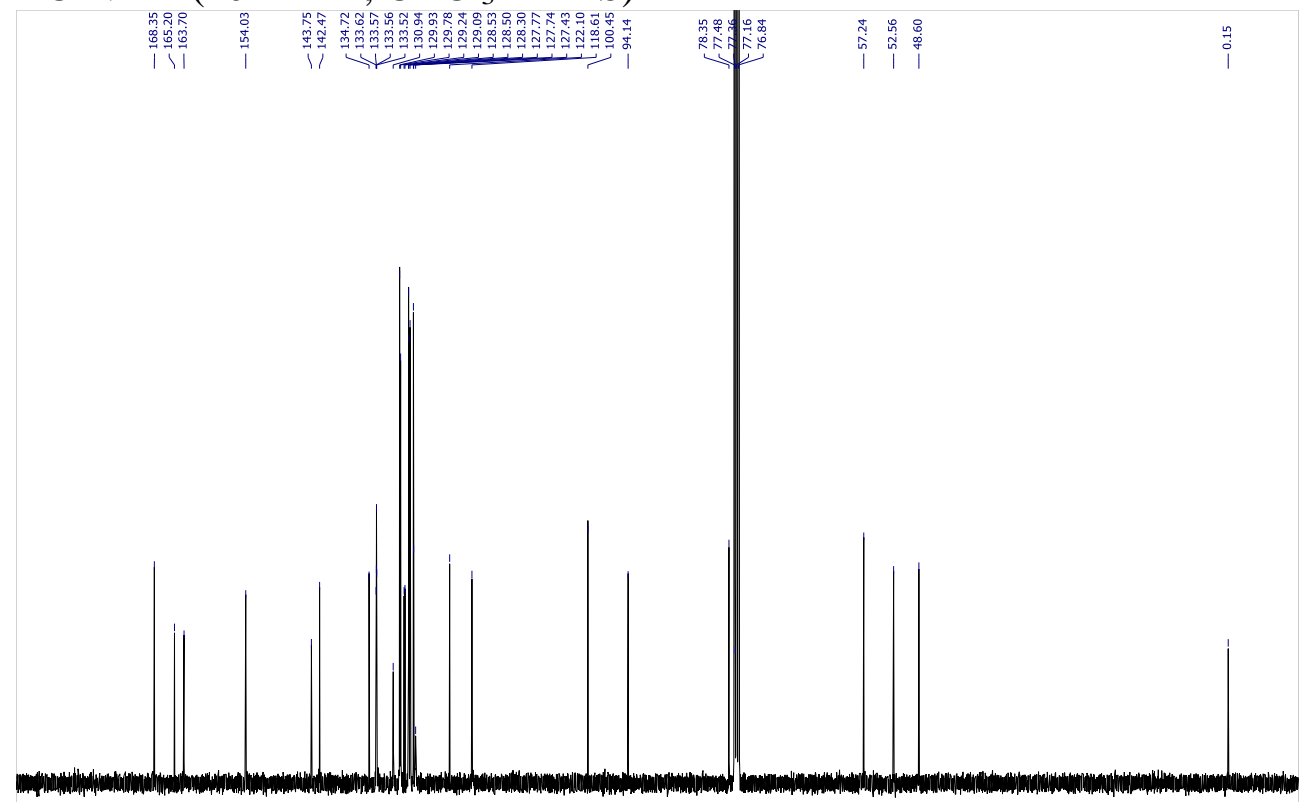

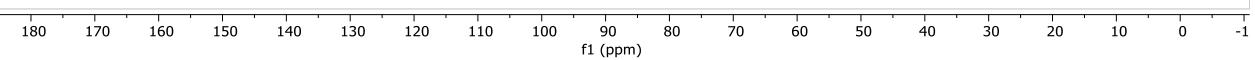


rac-(5a $R, 6 R, 8 \mathrm{a} R)-5 \mathrm{a}-(4-b r o m o p h e n y l)-3-c h l o r o-8 a-h y d r o x y-6-p h e n y l-5 a, 8 a-d i h y d r o-6 H-$ cyclopenta[4,5]furo[3,2-b]pyridine-7-carboxylic acid (S3)
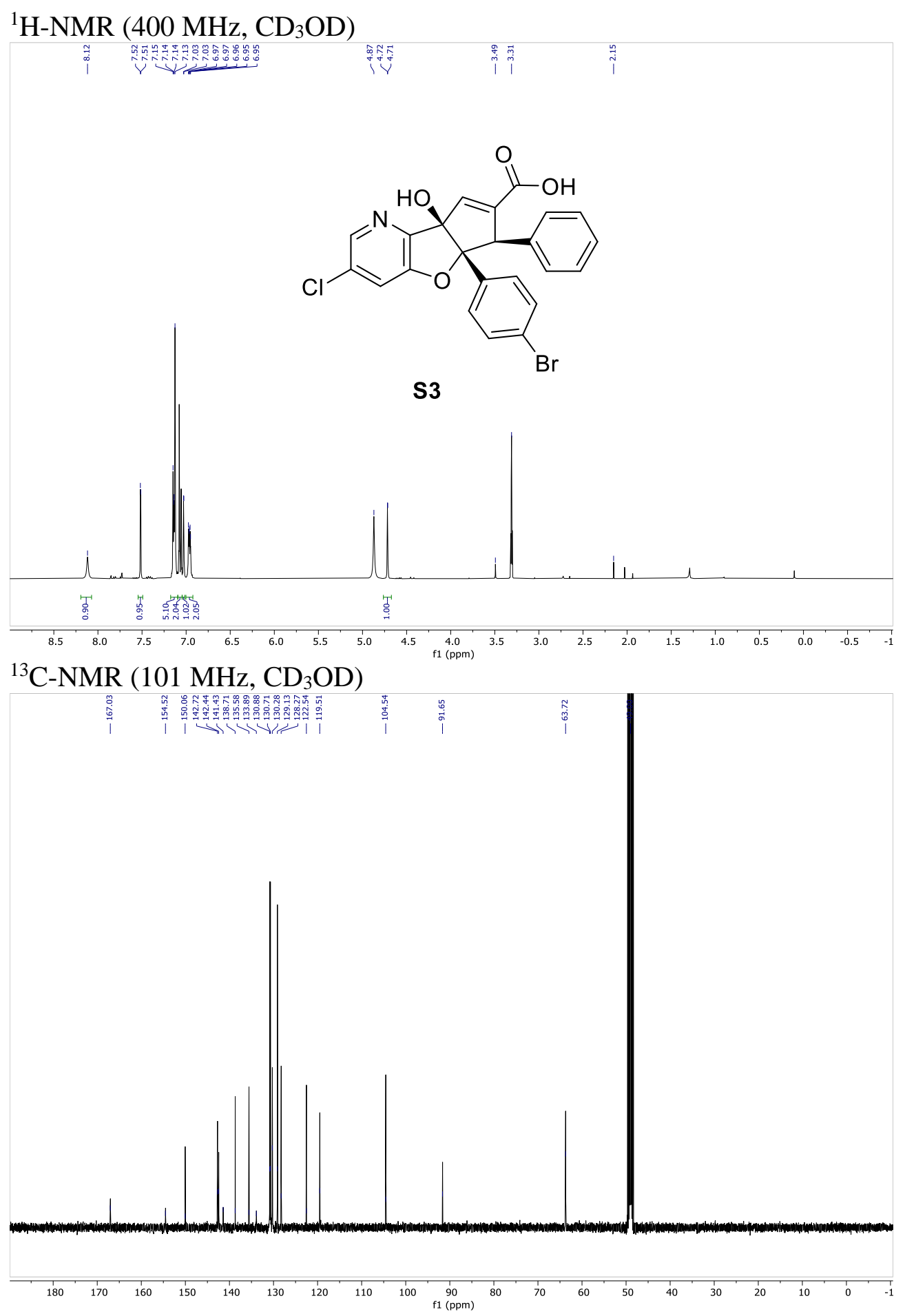

54 
$r a c$-methyl $(5 \mathrm{a} R, 6 R, 8 \mathrm{a} R)$-5a-(4-bromophenyl)-3-chloro-8a-hydroxy-6-phenyl-5a,8a-dihydro-6Hcyclopenta[4,5]furo[3,2-b]pyridine-7-carboxylate (20)
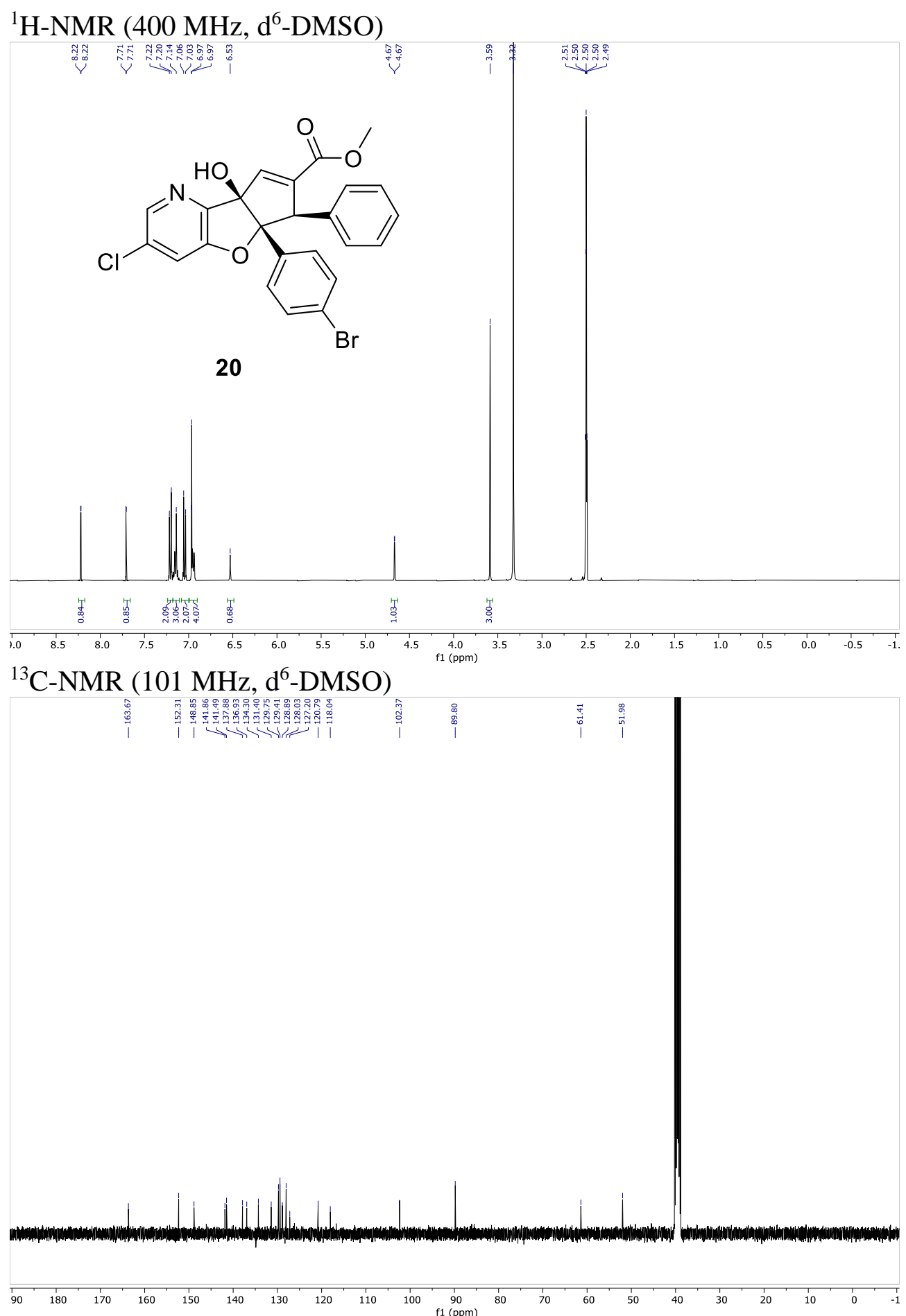
rac-methyl (5aR,6S,7R,8R,8aR)-3-chloro-8-cyano-5a-(4-cyanophenyl)-8a-hydroxy-6phenyl-5a,7,8,8a-tetrahydro-6H-cyclopenta[4,5]furo[3,2-b]pyridine-7-carboxylate (S4)

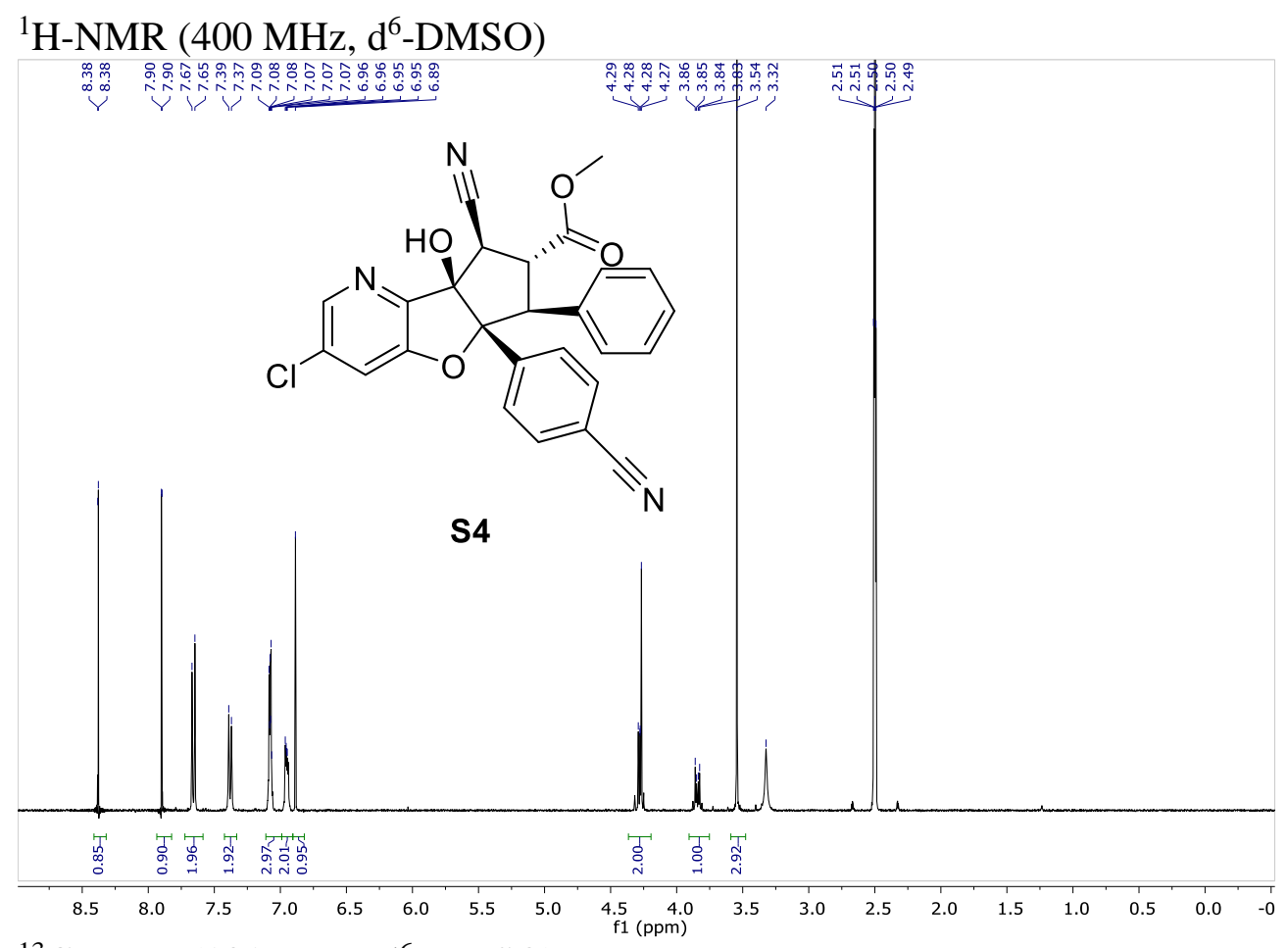

${ }^{13} \mathrm{C}-\mathrm{NMR}\left(101 \mathrm{MHz}, \mathrm{d}^{6}\right.$-DMSO)
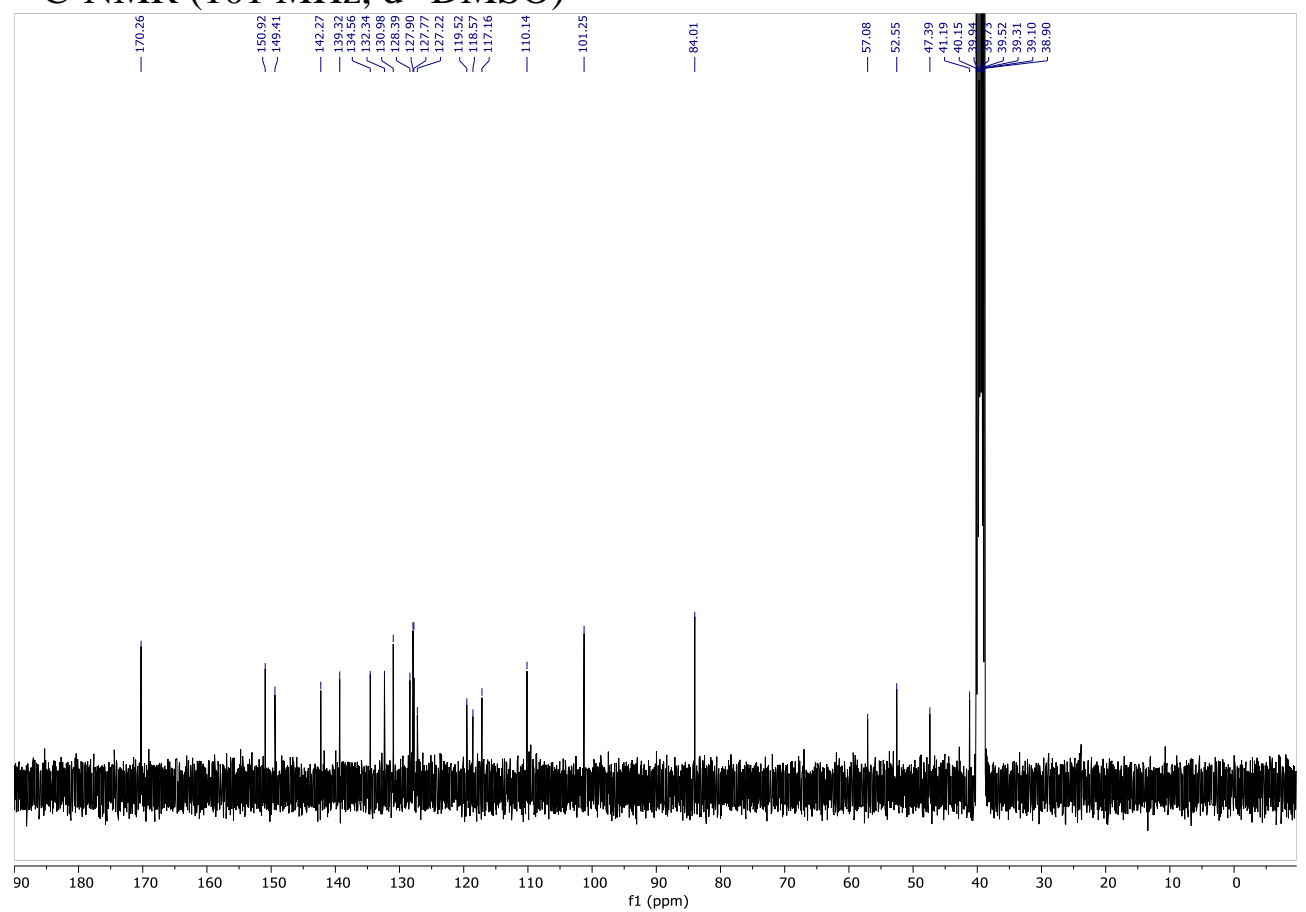
rac-(5aR,6S,7R,8R,8aR)-3-chloro-8-cyano-5a-(4-cyanophenyl)-8a-hydroxy-N,N-dimethyl-6phenyl-5a,7,8,8a-tetrahydro-6H-cyclopenta[4,5]furo[3,2-b]pyridine-7-carboxamide (26)

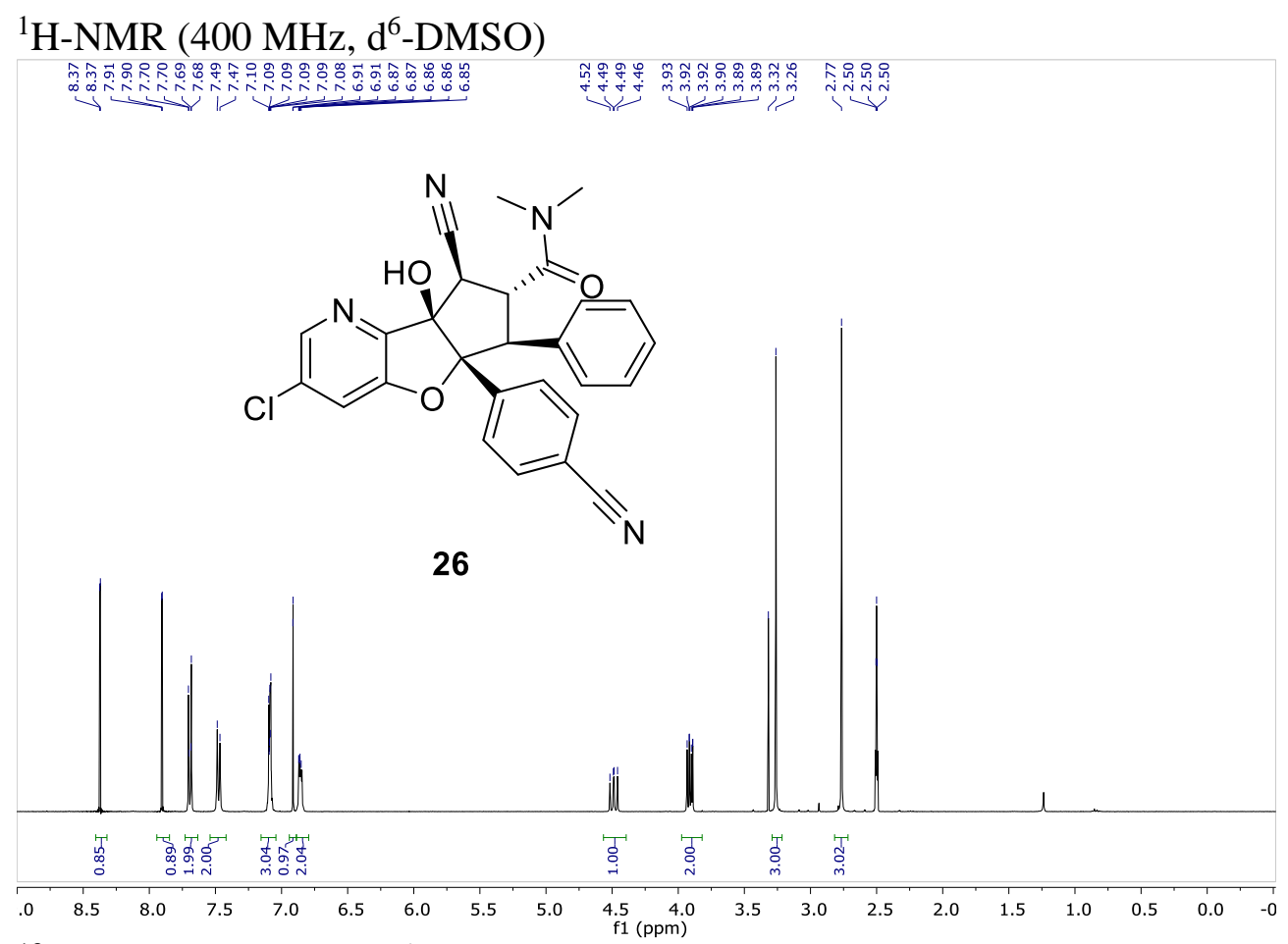

${ }^{13} \mathrm{C}-\mathrm{NMR}\left(101 \mathrm{MHz}, \mathrm{d}^{6}\right.$-DMSO)

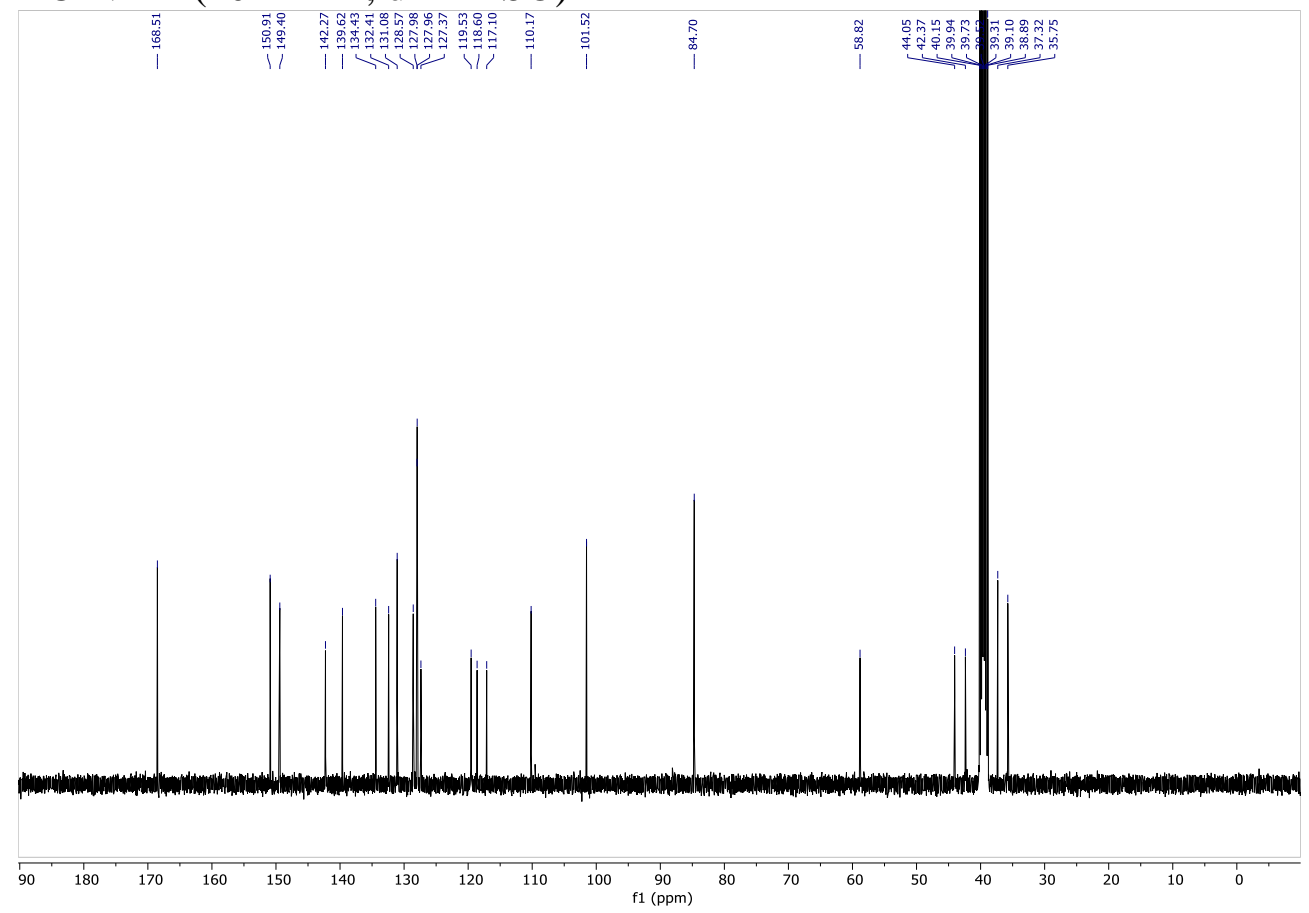




\section{X-ray crystallographic data}

\subsection{X-ray crystallographic data of compound $\mathbf{1 5}$}

\section{$\underline{\text { Experimental Summary }}$}

The single crystal X-ray diffraction studies were carried out on a Bruker Platinum-135 CCD diffractometer equipped with $\mathrm{Cu} \mathrm{K}_{\alpha}$ radiation $(\lambda=1.54178)$.

Crystal where used as received (grown from Metranol), A $0.20 \times 0.12 \times 0.06 \mathrm{~mm}$ colorless crystal was mounted on a Cryoloop with Paratone oil. Data were collected in a nitrogen gas stream at 100(2) K using $\phi$ and $\varpi$ scans. Crystal-to-detector distance was $45 \mathrm{~mm}$ using exposure time 1,2 and $4 \mathrm{~s}$ depending on the 2Theta angle with a scan width of $1.50^{\circ}$. Data collection was $98.50 \%$ complete to $67.50^{\circ}$ in $\theta$. A total of 18696 reflections were collected covering the indices, $-11<=\mathrm{h}<=11,-12<=\mathrm{k}<=12,-15<=\mathrm{l}<=15.4204$ reflections were found to be symmetry independent, with a $\mathrm{R}_{\text {int }}$ of 0.0388 . Indexing and unit cell refinement indicated a Primitive, Triclinic lattice. The space group was found to be $\boldsymbol{P - 1}$. The data were integrated using the Bruker SAINT software program and scaled using the SADABS software program. Solution by direct methods (SHELXT) produced a complete phasing model consistent with the proposed structure.

All nonhydrogen atoms were refined anisotropically by full-matrix least-squares (SHELXL-2014). All carbon bonded hydrogen atoms were placed using a riding model. Their positions were constrained relative to their parent atom using the appropriate HFIX command in SHELXL-2014.

Position of the O-H hydrogen atom have been refined using HFIX 147 command, with thermal parameter Uiso 1.5 of parent atom.

Crystallographic data are summarized in Table 1.

Notes:

Methanol solvate 


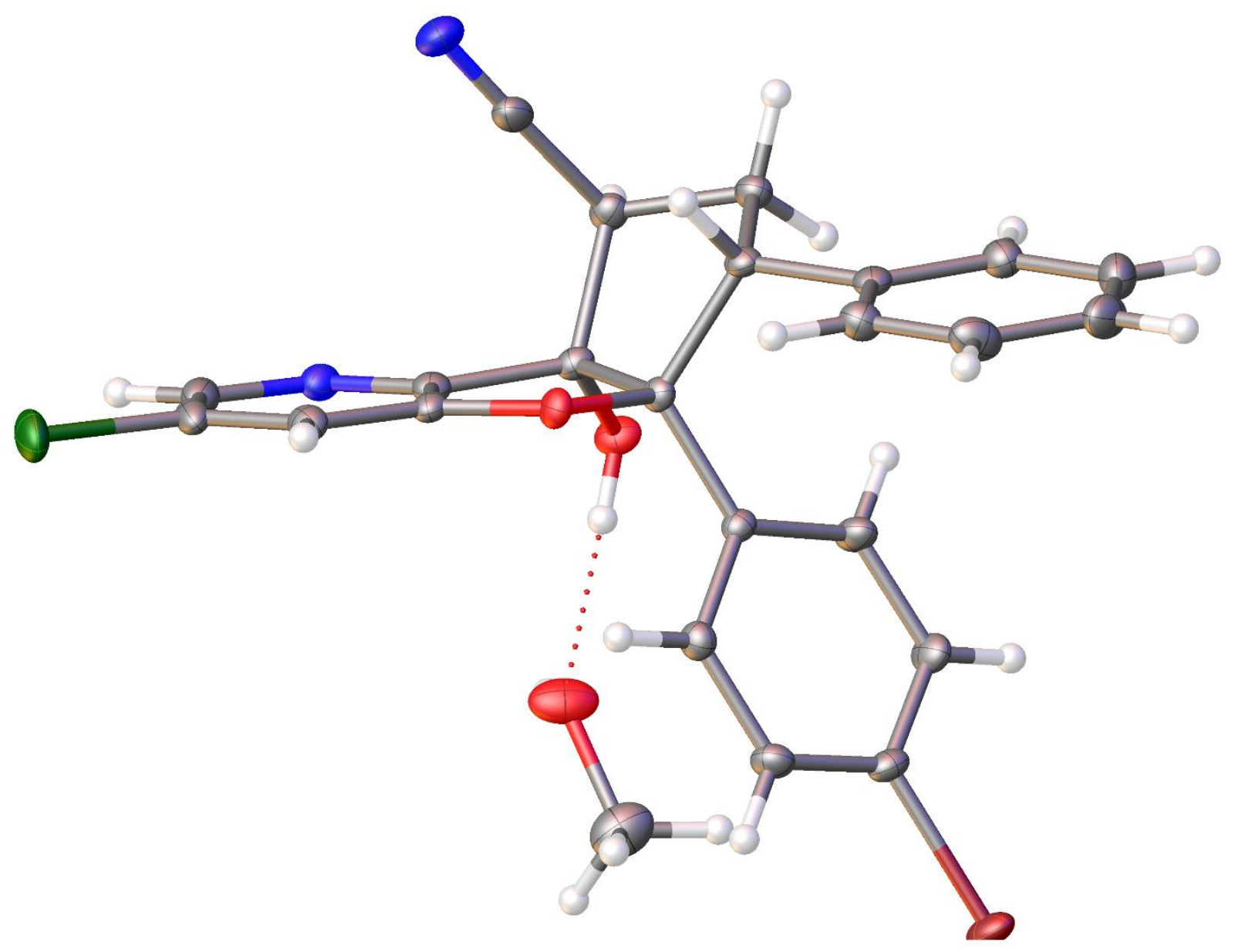




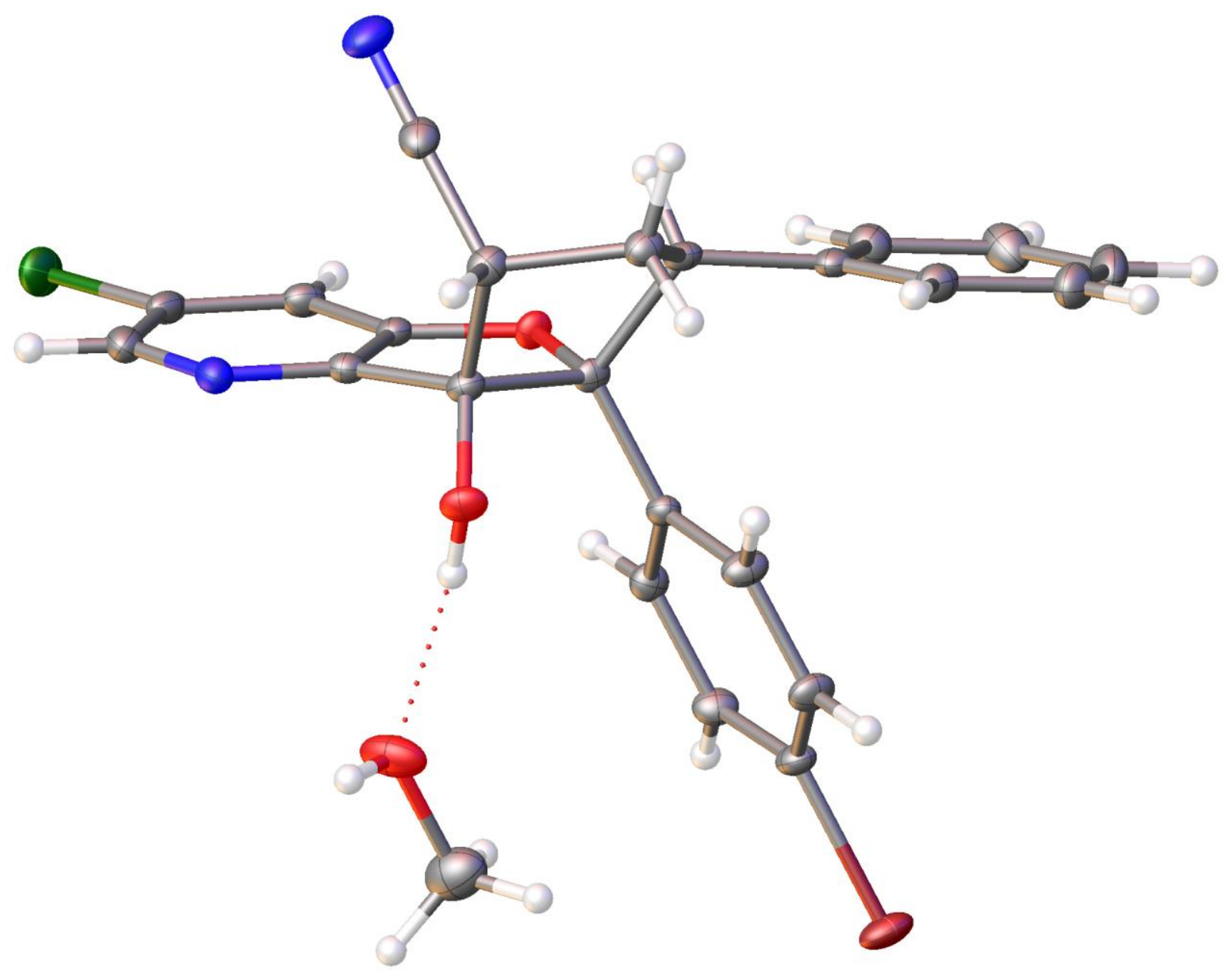


Table 1. Crystal data and structure refinement for CN_134_088.

Report date

Identification code

Empirical formula

Molecular formula

Formula weight

Temperature

Wavelength

Crystal system

Space group

Unit cell dimensions

Volume

Z

Density (calculated)

Absorption coefficient

$\mathrm{F}(000)$

Crystal size

Crystal color, habit

Theta range for data collection

Index ranges

Reflections collected

Independent reflections

Completeness to theta $=67.500^{\circ}$

Absorption correction

Max. and min. transmission

Refinement method

Data / restraints / parameters

Goodness-of-fit on $\mathrm{F}^{2}$

Final $\mathrm{R}$ indices [I>2sigma(I)]

$\mathrm{R}$ indices (all data)

Largest diff. peak and hole
2017-07-05

cn_134_088

$\mathrm{C} 24 \mathrm{H} 20 \mathrm{Br} \mathrm{Cl} \mathrm{N} 2 \mathrm{O} 3$

$\mathrm{C} 23 \mathrm{H} 16 \mathrm{Br} \mathrm{Cl} \mathrm{N} 2 \mathrm{O} 2, \mathrm{C} \mathrm{H} 4 \mathrm{O}$

499.78

$100.0 \mathrm{~K}$

$1.54178 \AA$

Triclinic

P-1

$\mathrm{a}=9.0883(2) \AA \quad \alpha=80.9870(10)^{\circ}$.

$\mathrm{b}=9.7340(2) \AA \quad \beta=77.1700(10)^{\circ}$.

$\mathrm{c}=12.7956(3) \AA \quad \gamma=87.6970(10)^{\circ}$.

1090.06(4) $\AA^{3}$

2

$1.523 \mathrm{Mg} / \mathrm{m}^{3}$

$3.939 \mathrm{~mm}^{-1}$

508

$0.2 \times 0.12 \times 0.06 \mathrm{~mm}^{3}$

colorless plank

3.584 to $72.758^{\circ}$.

$-11<=\mathrm{h}<=11,-12<=\mathrm{k}<=12,-15<=\mathrm{l}<=15$

18696

$4204[\mathrm{R}($ int $)=0.0388]$

$98.5 \%$

Semi-empirical from equivalents

0.5233 and 0.4092

Full-matrix least-squares on $\mathrm{F}^{2}$

4204 / 0 / 283

1.073

$\mathrm{R} 1=0.0278, \mathrm{wR} 2=0.0688$

$\mathrm{R} 1=0.0285, \mathrm{wR} 2=0.0693$

0.325 and -0.451 e. $\AA^{-3}$ 
Table 2. Atomic coordinates ( $\mathrm{x} 10^{4}$ ) and equivalent isotropic displacement parameters $\left(\AA^{2} \times 10^{3}\right)$ for $\mathrm{CN} \_134 \_088$. U(eq) is defined as one third of the trace of the orthogonalized $\mathrm{U}^{\mathrm{ij}}$ tensor.

\begin{tabular}{|c|c|c|c|c|}
\hline & $\mathrm{x}$ & y & $\mathrm{z}$ & $\mathrm{U}(\mathrm{eq})$ \\
\hline $\operatorname{Br}(1)$ & $5009(1)$ & $8897(1)$ & $3887(1)$ & $23(1)$ \\
\hline $\mathrm{Cl}(1)$ & 11313(1) & $-499(1)$ & $3026(1)$ & $27(1)$ \\
\hline $\mathrm{O}(1)$ & $6300(1)$ & $2168(1)$ & 3084(1) & $14(1)$ \\
\hline $\mathrm{O}(2)$ & $7320(1)$ & $4515(1)$ & $574(1)$ & $17(1)$ \\
\hline $\mathrm{O}(3)$ & $9134(2)$ & $6307(2)$ & 1086(1) & $30(1)$ \\
\hline $\mathrm{N}(1)$ & $9532(2)$ & 2092(2) & $926(1)$ & $18(1)$ \\
\hline $\mathrm{N}(2)$ & $6524(2)$ & $-207(2)$ & $600(2)$ & $27(1)$ \\
\hline $\mathrm{C}(1)$ & $10444(2)$ & 1214(2) & $1406(2)$ & $20(1)$ \\
\hline$C(2)$ & $10027(2)$ & $605(2)$ & $2488(2)$ & 19(1) \\
\hline$C(3)$ & $8640(2)$ & $867(2)$ & $3136(1)$ & $17(1)$ \\
\hline $\mathrm{C}(4)$ & $7714(2)$ & 1771(2) & $2618(1)$ & $14(1)$ \\
\hline$C(5)$ & $8192(2)$ & $2346(2)$ & 1541(1) & $15(1)$ \\
\hline$C(6)$ & $6935(2)$ & 3192(2) & $1168(1)$ & $14(1)$ \\
\hline$C(7)$ & $6119(2)$ & $2486(2)$ & $442(1)$ & $16(1)$ \\
\hline $\mathrm{C}(8)$ & $4441(2)$ & $2835(2)$ & $852(1)$ & $16(1)$ \\
\hline $\mathrm{C}(9)$ & $4260(2)$ & 2564(2) & 2089(1) & $14(1)$ \\
\hline$C(10)$ & $5726(2)$ & $3177(2)$ & $2284(1)$ & $12(1)$ \\
\hline $\mathrm{C}(11)$ & $6360(2)$ & $969(2)$ & 541(1) & 19(1) \\
\hline$C(12)$ & $5552(2)$ & $4566(2)$ & $2686(1)$ & $12(1)$ \\
\hline $\mathrm{C}(13)$ & $4828(2)$ & $5670(2)$ & 2163(1) & $18(1)$ \\
\hline$C(14)$ & $4657(2)$ & $6955(2)$ & 2514(1) & $18(1)$ \\
\hline$C(15)$ & $5225(2)$ & $7132(2)$ & $3402(1)$ & $16(1)$ \\
\hline$C(16)$ & $5962(2)$ & $6070(2)$ & 3931(1) & $17(1)$ \\
\hline $\mathrm{C}(17)$ & $6126(2)$ & 4788(2) & 3570(1) & $15(1)$ \\
\hline $\mathrm{C}(18)$ & 2793(2) & 2991(2) & 2783(1) & $16(1)$ \\
\hline $\mathrm{C}(19)$ & 1701(2) & $3807(2)$ & 2364(2) & 19(1) \\
\hline$C(20)$ & $362(2)$ & $4144(2)$ & 3051(2) & $25(1)$ \\
\hline $\mathrm{C}(21)$ & $95(2)$ & $3679(2)$ & $4155(2)$ & $26(1)$ \\
\hline$C(22)$ & $1180(2)$ & $2862(2)$ & $4579(2)$ & $25(1)$ \\
\hline$C(23)$ & $2510(2)$ & $2519(2)$ & 3899(1) & $19(1)$ \\
\hline$C(24)$ & $9024(3)$ & $7399(2)$ & 1714(2) & $34(1)$ \\
\hline
\end{tabular}


Table 3. Bond lengths $[\AA]$ and angles $\left[{ }^{\circ}\right]$ for $C N \_134 \_088$.

\begin{tabular}{|c|c|c|c|}
\hline $\mathrm{Br}(1)-\mathrm{C}(15)$ & $1.9050(16)$ & $\mathrm{C}(21)-\mathrm{H}(21)$ & 0.9500 \\
\hline $\mathrm{Cl}(1)-\mathrm{C}(2)$ & $1.7378(18)$ & $C(21)-C(22)$ & $1.392(3)$ \\
\hline $\mathrm{O}(1)-\mathrm{C}(4)$ & $1.359(2)$ & $\mathrm{C}(22)-\mathrm{H}(22)$ & 0.9500 \\
\hline $\mathrm{O}(1)-\mathrm{C}(10)$ & $1.4714(19)$ & $C(22)-C(23)$ & $1.386(3)$ \\
\hline $\mathrm{O}(2)-\mathrm{H}(2)$ & 0.8400 & $\mathrm{C}(23)-\mathrm{H}(23)$ & 0.9500 \\
\hline $\mathrm{O}(2)-\mathrm{C}(6)$ & $1.4039(19)$ & $\mathrm{C}(24)-\mathrm{H}(24 \mathrm{~A})$ & 0.9800 \\
\hline $\mathrm{O}(3)-\mathrm{H}(3 \mathrm{~A})$ & 0.8400 & $\mathrm{C}(24)-\mathrm{H}(24 \mathrm{~B})$ & 0.9800 \\
\hline $\mathrm{O}(3)-\mathrm{C}(24)$ & $1.418(3)$ & $\mathrm{C}(24)-\mathrm{H}(24 \mathrm{C})$ & 0.9800 \\
\hline $\mathrm{N}(1)-\mathrm{C}(1)$ & $1.341(2)$ & & \\
\hline $\mathrm{N}(1)-\mathrm{C}(5)$ & $1.332(2)$ & $\mathrm{C}(4)-\mathrm{O}(1)-\mathrm{C}(10)$ & $108.44(12)$ \\
\hline $\mathrm{N}(2)-\mathrm{C}(11)$ & $1.141(3)$ & $\mathrm{C}(6)-\mathrm{O}(2)-\mathrm{H}(2)$ & 109.5 \\
\hline $\mathrm{C}(1)-\mathrm{H}(1)$ & 0.9500 & $\mathrm{C}(24)-\mathrm{O}(3)-\mathrm{H}(3 \mathrm{~A})$ & 109.5 \\
\hline$C(1)-C(2)$ & $1.392(3)$ & $\mathrm{C}(5)-\mathrm{N}(1)-\mathrm{C}(1)$ & $116.26(15)$ \\
\hline$C(2)-C(3)$ & $1.385(3)$ & $\mathrm{N}(1)-\mathrm{C}(1)-\mathrm{H}(1)$ & 118.9 \\
\hline $\mathrm{C}(3)-\mathrm{H}(3)$ & 0.9500 & $\mathrm{~N}(1)-\mathrm{C}(1)-\mathrm{C}(2)$ & $122.27(16)$ \\
\hline$C(3)-C(4)$ & $1.389(2)$ & $\mathrm{C}(2)-\mathrm{C}(1)-\mathrm{H}(1)$ & 118.9 \\
\hline$C(4)-C(5)$ & $1.383(2)$ & $\mathrm{C}(1)-\mathrm{C}(2)-\mathrm{Cl}(1)$ & $118.11(14)$ \\
\hline$C(5)-C(6)$ & $1.501(2)$ & $\mathrm{C}(3)-\mathrm{C}(2)-\mathrm{Cl}(1)$ & $119.88(14)$ \\
\hline$C(6)-C(7)$ & $1.558(2)$ & $\mathrm{C}(3)-\mathrm{C}(2)-\mathrm{C}(1)$ & $122.00(16)$ \\
\hline$C(6)-C(10)$ & $1.596(2)$ & $\mathrm{C}(2)-\mathrm{C}(3)-\mathrm{H}(3)$ & 122.7 \\
\hline $\mathrm{C}(7)-\mathrm{H}(7)$ & 1.0000 & $C(2)-C(3)-C(4)$ & $114.58(16)$ \\
\hline $\mathrm{C}(7)-\mathrm{C}(8)$ & $1.541(2)$ & $\mathrm{C}(4)-\mathrm{C}(3)-\mathrm{H}(3)$ & 122.7 \\
\hline$C(7)-C(11)$ & $1.474(2)$ & $\mathrm{O}(1)-\mathrm{C}(4)-\mathrm{C}(3)$ & $125.13(15)$ \\
\hline $\mathrm{C}(8)-\mathrm{H}(8 \mathrm{~A})$ & 0.9900 & $\mathrm{O}(1)-\mathrm{C}(4)-\mathrm{C}(5)$ & $114.08(14)$ \\
\hline $\mathrm{C}(8)-\mathrm{H}(8 \mathrm{~B})$ & 0.9900 & $C(5)-C(4)-C(3)$ & $120.78(15)$ \\
\hline $\mathrm{C}(8)-\mathrm{C}(9)$ & $1.536(2)$ & $\mathrm{N}(1)-\mathrm{C}(5)-\mathrm{C}(4)$ & $124.11(16)$ \\
\hline $\mathrm{C}(9)-\mathrm{H}(9)$ & 1.0000 & $\mathrm{~N}(1)-\mathrm{C}(5)-\mathrm{C}(6)$ & $126.18(15)$ \\
\hline$C(9)-C(10)$ & $1.566(2)$ & $C(4)-C(5)-C(6)$ & $109.55(14)$ \\
\hline $\mathrm{C}(9)-\mathrm{C}(18)$ & $1.511(2)$ & $\mathrm{O}(2)-\mathrm{C}(6)-\mathrm{C}(5)$ & $116.39(13)$ \\
\hline $\mathrm{C}(10)-\mathrm{C}(12)$ & $1.511(2)$ & $\mathrm{O}(2)-\mathrm{C}(6)-\mathrm{C}(7)$ & $104.27(13)$ \\
\hline $\mathrm{C}(12)-\mathrm{C}(13)$ & $1.395(2)$ & $\mathrm{O}(2)-\mathrm{C}(6)-\mathrm{C}(10)$ & $115.38(13)$ \\
\hline$C(12)-C(17)$ & $1.394(2)$ & $\mathrm{C}(5)-\mathrm{C}(6)-\mathrm{C}(7)$ & $114.69(13)$ \\
\hline $\mathrm{C}(13)-\mathrm{H}(13)$ & 0.9500 & $\mathrm{C}(5)-\mathrm{C}(6)-\mathrm{C}(10)$ & $101.18(12)$ \\
\hline$C(13)-C(14)$ & $1.385(2)$ & $C(7)-C(6)-C(10)$ & $104.72(12)$ \\
\hline $\mathrm{C}(14)-\mathrm{H}(14)$ & 0.9500 & $\mathrm{C}(6)-\mathrm{C}(7)-\mathrm{H}(7)$ & 109.7 \\
\hline $\mathrm{C}(14)-\mathrm{C}(15)$ & $1.384(2)$ & $C(8)-C(7)-C(6)$ & $103.60(13)$ \\
\hline$C(15)-C(16)$ & $1.379(3)$ & $\mathrm{C}(8)-\mathrm{C}(7)-\mathrm{H}(7)$ & 109.7 \\
\hline $\mathrm{C}(16)-\mathrm{H}(16)$ & 0.9500 & $\mathrm{C}(11)-\mathrm{C}(7)-\mathrm{C}(6)$ & $113.27(14)$ \\
\hline$C(16)-C(17)$ & $1.389(2)$ & $\mathrm{C}(11)-\mathrm{C}(7)-\mathrm{H}(7)$ & 109.7 \\
\hline $\mathrm{C}(17)-\mathrm{H}(17)$ & 0.9500 & $\mathrm{C}(11)-\mathrm{C}(7)-\mathrm{C}(8)$ & $110.81(14)$ \\
\hline $\mathrm{C}(18)-\mathrm{C}(19)$ & $1.394(3)$ & $\mathrm{C}(7)-\mathrm{C}(8)-\mathrm{H}(8 \mathrm{~A})$ & 111.3 \\
\hline$C(18)-C(23)$ & $1.399(2)$ & $\mathrm{C}(7)-\mathrm{C}(8)-\mathrm{H}(8 \mathrm{~B})$ & 111.3 \\
\hline $\mathrm{C}(19)-\mathrm{H}(19)$ & 0.9500 & $\mathrm{H}(8 \mathrm{~A})-\mathrm{C}(8)-\mathrm{H}(8 \mathrm{~B})$ & 109.2 \\
\hline$C(19)-C(20)$ & $1.394(3)$ & $\mathrm{C}(9)-\mathrm{C}(8)-\mathrm{C}(7)$ & $102.53(13)$ \\
\hline $\mathrm{C}(20)-\mathrm{H}(20)$ & 0.9500 & $\mathrm{C}(9)-\mathrm{C}(8)-\mathrm{H}(8 \mathrm{~A})$ & 111.3 \\
\hline$C(20)-C(21)$ & $1.384(3)$ & $\mathrm{C}(9)-\mathrm{C}(8)-\mathrm{H}(8 \mathrm{~B})$ & 111.3 \\
\hline
\end{tabular}




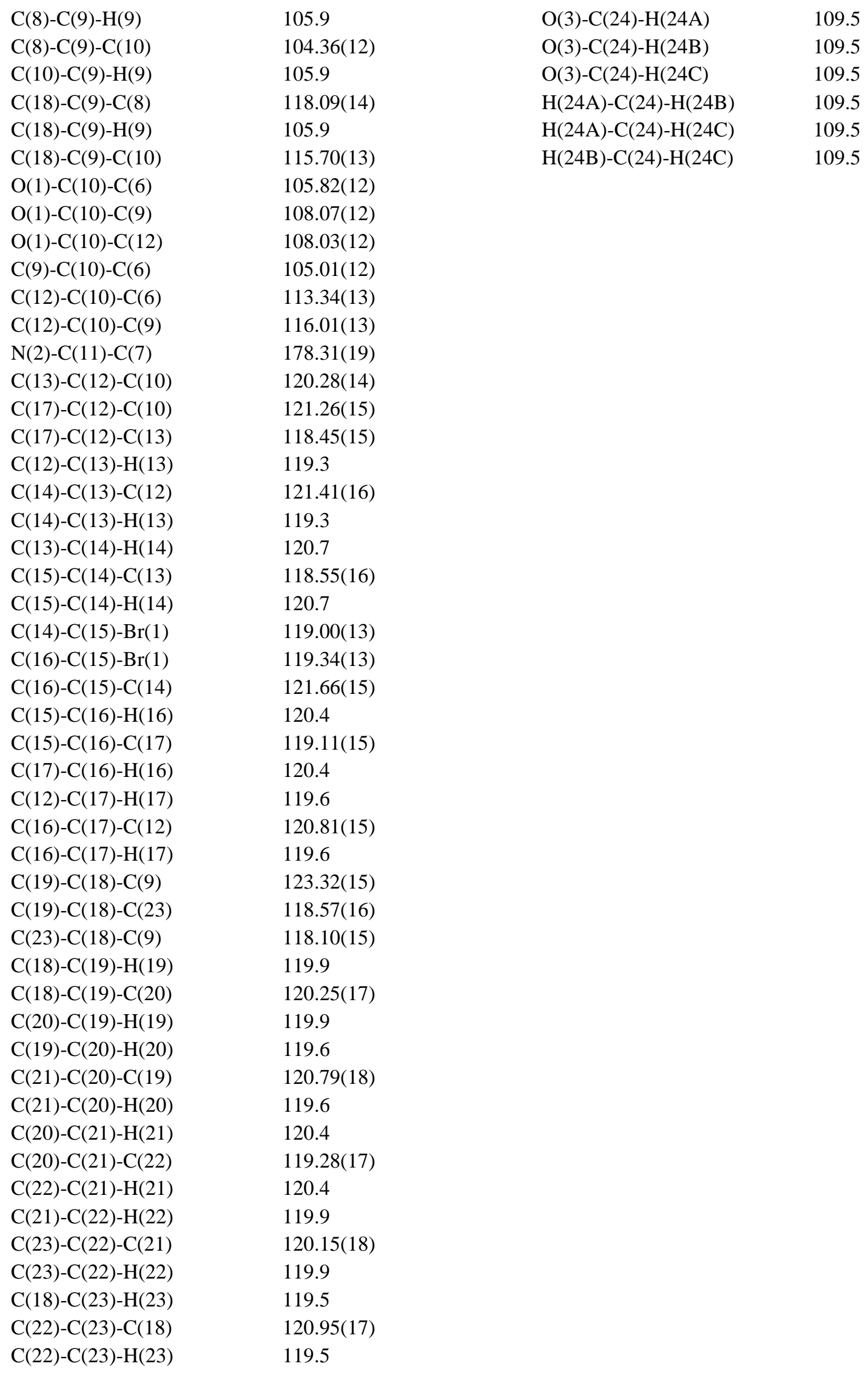


Table 4. Anisotropic displacement parameters $\left(\AA^{2} \times 10^{3}\right)$ for CN_134_088. The anisotropic displacement factor exponent takes the form: $-2 \pi^{2}\left[h^{2} a^{* 2} U^{11}+\ldots+2 h k a^{*} b^{*} U^{12}\right]$

\begin{tabular}{|c|c|c|c|c|c|c|}
\hline & $\mathrm{U}^{11}$ & $\mathrm{U}^{22}$ & $\mathrm{U}^{33}$ & $\mathrm{U}^{23}$ & $\mathrm{U}^{13}$ & $\mathrm{U}^{12}$ \\
\hline $\operatorname{Br}(1)$ & $33(1)$ & $12(1)$ & $27(1)$ & $-8(1)$ & $-13(1)$ & $3(1)$ \\
\hline $\mathrm{Cl}(1)$ & $19(1)$ & $21(1)$ & $42(1)$ & $-2(1)$ & $-13(1)$ & $6(1)$ \\
\hline $\mathrm{O}(1)$ & $16(1)$ & 11(1) & $13(1)$ & 1(1) & $-4(1)$ & $3(1)$ \\
\hline $\mathrm{O}(2)$ & $24(1)$ & $12(1)$ & $15(1)$ & $2(1)$ & $-4(1)$ & $-3(1)$ \\
\hline $\mathrm{O}(3)$ & $40(1)$ & $27(1)$ & $22(1)$ & $-1(1)$ & $-2(1)$ & $-16(1)$ \\
\hline $\mathrm{N}(1)$ & $15(1)$ & $16(1)$ & $22(1)$ & $-6(1)$ & $-2(1)$ & $-2(1)$ \\
\hline $\mathrm{N}(2)$ & $29(1)$ & $20(1)$ & $37(1)$ & $-14(1)$ & $-10(1)$ & $3(1)$ \\
\hline$C(1)$ & $14(1)$ & $17(1)$ & $30(1)$ & $-8(1)$ & $-4(1)$ & $-1(1)$ \\
\hline$C(2)$ & $16(1)$ & 11(1) & $32(1)$ & $-5(1)$ & $-10(1)$ & 1(1) \\
\hline$C(3)$ & $19(1)$ & $12(1)$ & 21(1) & $-1(1)$ & $-7(1)$ & $-1(1)$ \\
\hline$C(4)$ & $14(1)$ & $10(1)$ & $18(1)$ & $-4(1)$ & $-4(1)$ & $-1(1)$ \\
\hline $\mathrm{C}(5)$ & $15(1)$ & $12(1)$ & $18(1)$ & $-3(1)$ & $-4(1)$ & $-2(1)$ \\
\hline$C(6)$ & $17(1)$ & 11(1) & $13(1)$ & $-2(1)$ & $-3(1)$ & $-1(1)$ \\
\hline$C(7)$ & $19(1)$ & $16(1)$ & $14(1)$ & $-3(1)$ & $-6(1)$ & 2(1) \\
\hline $\mathrm{C}(8)$ & $18(1)$ & $15(1)$ & $17(1)$ & $-4(1)$ & $-7(1)$ & 1(1) \\
\hline $\mathrm{C}(9)$ & $16(1)$ & $9(1)$ & $17(1)$ & $-3(1)$ & $-6(1)$ & $-1(1)$ \\
\hline $\mathrm{C}(10)$ & $15(1)$ & $10(1)$ & $12(1)$ & $0(1)$ & $-5(1)$ & 1(1) \\
\hline$C(11)$ & $17(1)$ & $22(1)$ & $20(1)$ & $-10(1)$ & $-6(1)$ & $1(1)$ \\
\hline $\mathrm{C}(12)$ & $13(1)$ & $10(1)$ & $14(1)$ & $-1(1)$ & $-2(1)$ & $-1(1)$ \\
\hline$C(13)$ & $24(1)$ & $14(1)$ & $17(1)$ & $-4(1)$ & $-10(1)$ & $1(1)$ \\
\hline $\mathrm{C}(14)$ & $23(1)$ & $12(1)$ & $22(1)$ & $-1(1)$ & $-10(1)$ & $2(1)$ \\
\hline $\mathrm{C}(15)$ & $18(1)$ & 11(1) & $17(1)$ & $-4(1)$ & $-2(1)$ & $-2(1)$ \\
\hline$C(16)$ & 21(1) & $17(1)$ & $16(1)$ & $-4(1)$ & $-7(1)$ & $-2(1)$ \\
\hline $\mathrm{C}(17)$ & $16(1)$ & $14(1)$ & $16(1)$ & $-1(1)$ & $-5(1)$ & 1(1) \\
\hline $\mathrm{C}(18)$ & $16(1)$ & 11(1) & $21(1)$ & $-5(1)$ & $-5(1)$ & $-2(1)$ \\
\hline $\mathrm{C}(19)$ & $20(1)$ & $16(1)$ & $24(1)$ & $-4(1)$ & $-8(1)$ & 1(1) \\
\hline $\mathrm{C}(20)$ & $18(1)$ & $22(1)$ & $36(1)$ & $-8(1)$ & $-9(1)$ & $4(1)$ \\
\hline $\mathrm{C}(21)$ & $16(1)$ & $30(1)$ & $33(1)$ & $-16(1)$ & $-1(1)$ & $1(1)$ \\
\hline $\mathrm{C}(22)$ & $22(1)$ & $33(1)$ & $20(1)$ & $-9(1)$ & $-2(1)$ & $-5(1)$ \\
\hline $\mathrm{C}(23)$ & $19(1)$ & $20(1)$ & $20(1)$ & $-4(1)$ & $-6(1)$ & $-1(1)$ \\
\hline$C(24)$ & $43(1)$ & $28(1)$ & $29(1)$ & $-4(1)$ & $-2(1)$ & $-3(1)$ \\
\hline
\end{tabular}


Table 5. Hydrogen coordinates ( x 10 $)$ and isotropic displacement parameters $\left(\AA^{2} \times 10^{3}\right)$ for CN_134_088.

\begin{tabular}{|c|c|c|c|c|}
\hline & $\mathrm{x}$ & $\mathrm{y}$ & $\mathrm{z}$ & $\mathrm{U}(\mathrm{eq})$ \\
\hline $\mathrm{H}(2)$ & 7770 & 4959 & 921 & 26 \\
\hline $\mathrm{H}(3 \mathrm{~A})$ & 9594 & 6591 & 447 & 45 \\
\hline $\mathrm{H}(1)$ & 11407 & 1001 & 994 & 24 \\
\hline $\mathrm{H}(3)$ & 8347 & 462 & 3876 & 20 \\
\hline $\mathrm{H}(7)$ & 6461 & 2919 & -333 & 19 \\
\hline $\mathrm{H}(8 \mathrm{~A})$ & 4215 & 3817 & 596 & 19 \\
\hline $\mathrm{H}(8 \mathrm{~B})$ & 3778 & 2222 & 613 & 19 \\
\hline $\mathrm{H}(9)$ & 4337 & 1532 & 2289 & 16 \\
\hline $\mathrm{H}(13)$ & 4444 & 5537 & 1554 & 21 \\
\hline $\mathrm{H}(14)$ & 4160 & 7698 & 2153 & 22 \\
\hline $\mathrm{H}(16)$ & 6354 & 6214 & 4535 & 21 \\
\hline $\mathrm{H}(17)$ & 6634 & 4052 & 3929 & 18 \\
\hline H(19) & 1869 & 4134 & 1608 & 23 \\
\hline $\mathrm{H}(20)$ & -376 & 4700 & 2757 & 29 \\
\hline $\mathrm{H}(21)$ & -818 & 3915 & 4619 & 31 \\
\hline $\mathrm{H}(22)$ & 1008 & 2538 & 5335 & 30 \\
\hline $\mathrm{H}(23)$ & 3240 & 1954 & 4195 & 23 \\
\hline $\mathrm{H}(24 \mathrm{~A})$ & 9987 & 7893 & 1539 & 51 \\
\hline $\mathrm{H}(24 \mathrm{~B})$ & 8785 & 7011 & 2486 & 51 \\
\hline $\mathrm{H}(24 \mathrm{C})$ & 8223 & 8048 & 1552 & 51 \\
\hline
\end{tabular}




\subsection{X-ray crystallographic data of compound $\mathbf{2 6}$}

\section{$\underline{\text { Experimental Summary }}$}

The single crystal X-ray diffraction studies were carried out on a Bruker Kappa APEX-II CCD diffractometer equipped with $\mathrm{Cu} \mathrm{K}_{\alpha}$ radiation $(\lambda=1.5478)$. Crystals of the subject compound were grown by dissolving approximately $1 \mathrm{mg}$ of sample in $350 \mu \mathrm{L}$ of Dichloroethane, which was then vapor diffused with Pentane over several days. A $0.135 \times 0.067 \times 0.041 \mathrm{~mm}$ piece if a colorless rod was mounted on a Cryoloop with Paratone oil. Data were collected in a nitrogen gas stream at 100(2) K using $\phi$ and $\varpi$ scans. Crystal-to-detector distance was $40 \mathrm{~mm}$ using variable exposure time (5s-20s) depending on $\theta$ with a scan width of $1.0^{\circ}$. Data collection was $98.8 \%$ complete to $68.00^{\circ}$ in $\theta$. A total of 97780 reflections were collected covering the indices, $-21<=\mathrm{h}<=22,-23<=\mathrm{k}<=23,-24<=1<=22.14480$ reflections were found to be symmetry independent, with a $\mathrm{R}_{\text {int }}$ of 0.0897 . Indexing and unit cell refinement indicated a primitive, monoclinic lattice. The space group was found to be $P 2_{1} / \mathrm{n}$. The data were integrated using the Bruker SAINT software program and scaled using the SADABS software program. Solution by direct methods (SHELXT) produced a complete phasing model consistent with the proposed structure.

All nonhydrogen atoms were refined anisotropically by full-matrix least-squares (SHELXL-2014). All hydrogen atoms were placed using a riding model. Their positions were constrained relative to their parent atom using the appropriate HFIX command in SHELXL-2014. Crystallographic data are summarized in Table 1. 


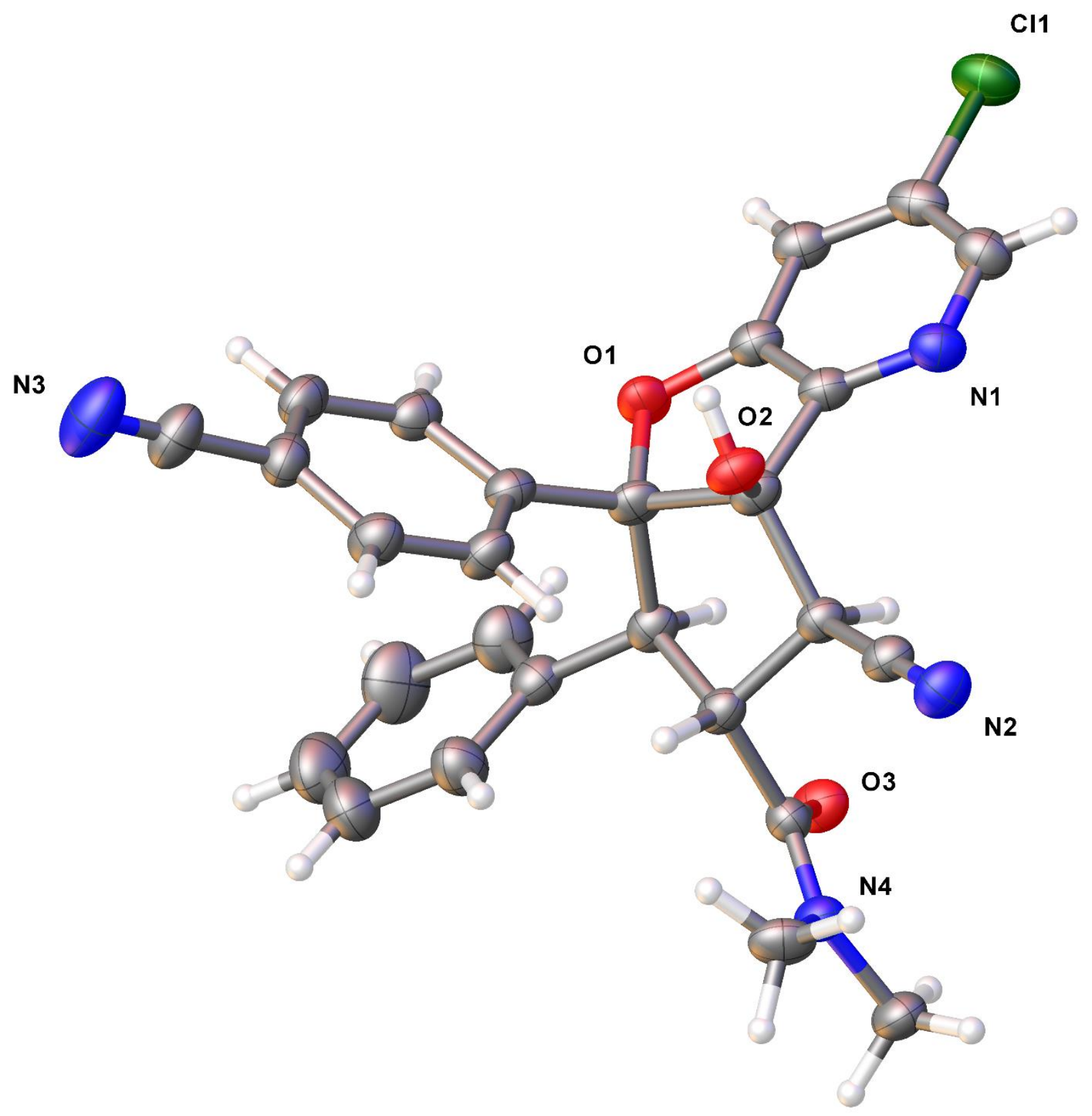


Table 1. Crystal data and structure refinement for eFT1605.

\begin{tabular}{|c|c|c|}
\hline Report date & \multicolumn{2}{|l|}{$2016-10-20$} \\
\hline Identification code & \multicolumn{2}{|l|}{ eFT1605 } \\
\hline Empirical formula & \multicolumn{2}{|l|}{$\mathrm{C} 27 \mathrm{H} 21 \mathrm{Cl} \mathrm{N} 4 \mathrm{O} 3$} \\
\hline Molecular formula & \multicolumn{2}{|l|}{$\mathrm{C} 27 \mathrm{H} 21 \mathrm{Cl} \mathrm{N} 4 \mathrm{O} 3$} \\
\hline Formula weight & \multicolumn{2}{|l|}{484.93} \\
\hline Temperature & \multicolumn{2}{|l|}{$100.0 \mathrm{~K}$} \\
\hline Wavelength & \multicolumn{2}{|l|}{$1.54178 \AA$} \\
\hline Crystal system & \multicolumn{2}{|l|}{ Monoclinic } \\
\hline Space group & \multicolumn{2}{|l|}{ P $121 / n 1$} \\
\hline \multirow[t]{3}{*}{ Unit cell dimensions } & $a=19.5956(4) \AA$ & $\alpha=90^{\circ}$ \\
\hline & $\mathrm{b}=19.9085(4) \AA$ & $\beta=93.891(2)^{\circ}$. \\
\hline & $c=20.5971(4) \AA$ & $\gamma=90^{\circ}$ \\
\hline Volume & \multicolumn{2}{|l|}{$8016.8(3) \AA^{3}$} \\
\hline $\mathrm{Z}$ & \multicolumn{2}{|l|}{12} \\
\hline Density (calculated) & \multicolumn{2}{|l|}{$1.205 \mathrm{Mg} / \mathrm{m}^{3}$} \\
\hline Absorption coefficient & \multicolumn{2}{|l|}{$1.540 \mathrm{~mm}^{-1}$} \\
\hline $\mathrm{F}(000)$ & \multicolumn{2}{|l|}{3024} \\
\hline Crystal size & \multicolumn{2}{|c|}{$0.135 \times 0.067 \times 0.041 \mathrm{~mm}^{3}$} \\
\hline Crystal color, habit & \multicolumn{2}{|l|}{ Colorless Rod } \\
\hline Theta range for data collection & \multicolumn{2}{|l|}{3.012 to $68.322^{\circ}$. } \\
\hline Index ranges & \multicolumn{2}{|c|}{$-21<=\mathrm{h}<=22,-23<=\mathrm{k}<=23,-24<=1<=22$} \\
\hline Reflections collected & \multicolumn{2}{|l|}{97780} \\
\hline Independent reflections & \multicolumn{2}{|c|}{$14480[\mathrm{R}(\mathrm{int})=0.0897, \mathrm{R}($ sigma $)=0.0462]$} \\
\hline Completeness to theta $=68.000^{\circ}$ & \multicolumn{2}{|l|}{$98.8 \%$} \\
\hline Absorption correction & \multicolumn{2}{|c|}{ Semi-empirical from equivalents } \\
\hline Max. and min. transmission & \multicolumn{2}{|l|}{0.3201 and 0.2231} \\
\hline Refinement method & \multicolumn{2}{|c|}{ Full-matrix least-squares on $\mathrm{F}^{2}$} \\
\hline Data / restraints / parameters & \multicolumn{2}{|l|}{14480 / 0 / 955} \\
\hline Goodness-of-fit on $\mathrm{F}^{2}$ & \multicolumn{2}{|l|}{1.012} \\
\hline Final $R$ indices $[\mathrm{I}>2 \operatorname{sigma}(\mathrm{I})]$ & \multicolumn{2}{|c|}{$\mathrm{R} 1=0.0395, \mathrm{wR} 2=0.0921$} \\
\hline $\mathrm{R}$ indices (all data) & \multicolumn{2}{|c|}{$\mathrm{R} 1=0.0650, \mathrm{wR} 2=0.1031$} \\
\hline Extinction coefficient & \multicolumn{2}{|l|}{$\mathrm{n} / \mathrm{a}$} \\
\hline Largest diff. peak and hole & \multicolumn{2}{|c|}{0.230 and -0.289 e. $\AA^{-3}$} \\
\hline
\end{tabular}


Table 2. Atomic coordinates ( $\times 10^{4}$ ) and equivalent isotropic displacement parameters $\left(\AA^{2} \times 10^{3}\right)$ for eFT1605. U(eq) is defined as one third of the trace of the orthogonalized $\mathrm{U}^{\mathrm{ij}}$ tensor.

\begin{tabular}{|c|c|c|c|c|}
\hline & $\mathrm{x}$ & $\mathrm{y}$ & $\mathrm{z}$ & $\mathrm{U}(\mathrm{eq})$ \\
\hline $\mathrm{Cl}(1)$ & 7872(1) & $3671(1)$ & $3443(1)$ & $42(1)$ \\
\hline $\mathrm{O}(1)$ & $5421(1)$ & $2760(1)$ & $3486(1)$ & $33(1)$ \\
\hline $\mathrm{O}(2)$ & 4999(1) & $3498(1)$ & 4947(1) & $32(1)$ \\
\hline $\mathrm{O}(3)$ & 5491(1) & $835(1)$ & 4881(1) & $37(1)$ \\
\hline $\mathrm{N}(1)$ & $6527(1)$ & $3426(1)$ & 4773(1) & $34(1)$ \\
\hline $\mathrm{N}(2)$ & $5342(1)$ & 2744(1) & $6350(1)$ & $38(1)$ \\
\hline $\mathrm{N}(3)$ & 1940(1) & $4263(1)$ & $3125(1)$ & $64(1)$ \\
\hline $\mathrm{N}(4)$ & $4853(1)$ & $980(1)$ & $5746(1)$ & $32(1)$ \\
\hline $\mathrm{C}(1)$ & 7088(1) & $3570(1)$ & 4459(1) & $36(1)$ \\
\hline$C(2)$ & 7121(1) & $3456(1)$ & 3799(1) & $34(1)$ \\
\hline$C(3)$ & $6584(1)$ & $3182(1)$ & $3422(1)$ & $32(1)$ \\
\hline C(4) & 6011(1) & $3032(1)$ & $3754(1)$ & $31(1)$ \\
\hline$C(5)$ & $6008(1)$ & $3162(1)$ & 4411(1) & $30(1)$ \\
\hline$C(6)$ & $5330(1)$ & 2967(1) & 4652(1) & $29(1)$ \\
\hline$C(7)$ & 4957(1) & $2652(1)$ & 4010(1) & $30(1)$ \\
\hline $\mathrm{C}(8)$ & 4934(1) & 1882(1) & 4152(1) & $29(1)$ \\
\hline $\mathrm{C}(9)$ & $4860(1)$ & $1846(1)$ & $4888(1)$ & $28(1)$ \\
\hline$C(10)$ & $5392(1)$ & $2372(1)$ & $5138(1)$ & $28(1)$ \\
\hline$C(11)$ & $5355(1)$ & $2588(1)$ & 5818(1) & $31(1)$ \\
\hline $\mathrm{C}(12)$ & $4273(1)$ & $2956(1)$ & $3790(1)$ & $30(1)$ \\
\hline$C(13)$ & $4113(1)$ & $3111(1)$ & $3137(1)$ & $33(1)$ \\
\hline$C(14)$ & $3498(1)$ & $3416(1)$ & 2941(1) & $36(1)$ \\
\hline$C(15)$ & $3035(1)$ & $3566(1)$ & $3402(1)$ & $36(1)$ \\
\hline$C(16)$ & $3170(1)$ & $3377(1)$ & $4046(1)$ & $38(1)$ \\
\hline $\mathrm{C}(17)$ & $3785(1)$ & $3075(1)$ & $4236(1)$ & $35(1)$ \\
\hline$C(18)$ & $2420(1)$ & $3946(1)$ & $3234(1)$ & $45(1)$ \\
\hline$C(19)$ & $4434(1)$ & $1485(1)$ & $3717(1)$ & $33(1)$ \\
\hline $\mathrm{C}(20)$ & $4633(1)$ & $1257(1)$ & $3123(1)$ & $47(1)$ \\
\hline $\mathrm{C}(21)$ & $4186(2)$ & $902(2)$ & 2704(1) & $62(1)$ \\
\hline $\mathrm{C}(22)$ & $3529(2)$ & $768(1)$ & $2867(1)$ & $59(1)$ \\
\hline$C(23)$ & $3324(1)$ & 992(1) & $3450(1)$ & $52(1)$ \\
\hline$C(24)$ & $3772(1)$ & $1347(1)$ & 3877(1) & $44(1)$ \\
\hline
\end{tabular}




\begin{tabular}{|c|c|c|c|c|}
\hline $\mathrm{C}(25)$ & $5073(1)$ & $1164(1)$ & $5174(1)$ & $28(1)$ \\
\hline$C(26)$ & $5127(1)$ & $374(1)$ & 6071(1) & $39(1)$ \\
\hline$C(27)$ & $4342(1)$ & $1337(1)$ & 6101(1) & $40(1)$ \\
\hline $\mathrm{Cl}\left(1^{\prime}\right)$ & $7265(1)$ & $2675(1)$ & $1606(1)$ & $43(1)$ \\
\hline $\mathrm{O}\left(1^{\prime}\right)$ & 6493(1) & $5051(1)$ & 2184(1) & $30(1)$ \\
\hline $\mathrm{O}\left(2^{\prime}\right)$ & 4921(1) & $5019(1)$ & 1471(1) & $31(1)$ \\
\hline $\mathrm{O}\left(3^{\prime}\right)$ & $5089(1)$ & $4431(1)$ & $4048(1)$ & $37(1)$ \\
\hline $\mathrm{N}\left(1^{\prime}\right)$ & $5494(1)$ & $3590(1)$ & 1811(1) & $34(1)$ \\
\hline $\mathrm{N}\left(2^{\prime}\right)$ & $3483(1)$ & 4984(1) & 2152(1) & $45(1)$ \\
\hline $\mathrm{N}\left(3^{\prime}\right)$ & $5592(1)$ & $8723(1)$ & 2044(1) & $51(1)$ \\
\hline $\mathrm{N}\left(4^{\prime}\right)$ & $4225(1)$ & $5186(1)$ & 4061(1) & $32(1)$ \\
\hline$C\left(1^{\prime}\right)$ & $5985(1)$ & $3140(1)$ & 1694(1) & $37(1)$ \\
\hline $\mathrm{C}\left(2^{\prime}\right)$ & 6674(1) & $3303(1)$ & $1735(1)$ & $35(1)$ \\
\hline$C\left(3^{\prime}\right)$ & 6903(1) & $3941(1)$ & 1903(1) & $31(1)$ \\
\hline$C\left(4^{\prime}\right)$ & 6393(1) & 4398(1) & 2015(1) & $30(1)$ \\
\hline$C\left(5^{\prime}\right)$ & $5715(1)$ & $4205(1)$ & 1960(1) & $30(1)$ \\
\hline$C\left(6^{\prime}\right)$ & $5270(1)$ & $4817(1)$ & $2055(1)$ & $29(1)$ \\
\hline$C\left(7^{\prime}\right)$ & $5829(1)$ & 5301(1) & $2366(1)$ & $29(1)$ \\
\hline$C\left(8^{\prime}\right)$ & $5771(1)$ & $5167(1)$ & $3110(1)$ & $28(1)$ \\
\hline $\mathrm{C}\left(9^{\prime}\right)$ & 4998(1) & $5189(1)$ & $3162(1)$ & $29(1)$ \\
\hline $\mathrm{C}\left(10^{\prime}\right)$ & $4740(1)$ & $4732(1)$ & 2583(1) & $31(1)$ \\
\hline $\mathrm{C}\left(11^{\prime}\right)$ & $4040(1)$ & $4875(1)$ & 2333(1) & $35(1)$ \\
\hline $\mathrm{C}\left(12^{\prime}\right)$ & $5782(1)$ & $6038(1)$ & $2198(1)$ & $28(1)$ \\
\hline $\mathrm{C}\left(13^{\prime}\right)$ & $5148(1)$ & 6354(1) & 2102(1) & $30(1)$ \\
\hline$C\left(14^{\prime}\right)$ & $5100(1)$ & $7046(1)$ & $2050(1)$ & $32(1)$ \\
\hline$C\left(15^{\prime}\right)$ & $5695(1)$ & $7429(1)$ & 2081(1) & $32(1)$ \\
\hline$C\left(16^{\prime}\right)$ & 6334(1) & $7119(1)$ & 2151(1) & $33(1)$ \\
\hline$C\left(17^{\prime}\right)$ & 6372(1) & $6430(1)$ & 2211(1) & $31(1)$ \\
\hline $\mathrm{C}\left(18^{\prime}\right)$ & $5641(1)$ & $8152(1)$ & $2054(1)$ & $38(1)$ \\
\hline $\mathrm{C}\left(19^{\prime}\right)$ & $6221(1)$ & $5608(1)$ & $3551(1)$ & $29(1)$ \\
\hline $\mathrm{C}\left(20^{\prime}\right)$ & 6033(1) & $6252(1)$ & $3726(1)$ & $34(1)$ \\
\hline$C\left(21^{\prime}\right)$ & 6491(1) & $6664(1)$ & $4075(1)$ & $40(1)$ \\
\hline $\mathrm{C}\left(22^{\prime}\right)$ & $7140(1)$ & $6438(1)$ & $4264(1)$ & $44(1)$ \\
\hline$C\left(23^{\prime}\right)$ & $7332(1)$ & $5794(1)$ & $4107(1)$ & $44(1)$ \\
\hline$C\left(24^{\prime}\right)$ & 6874(1) & $5382(1)$ & $3752(1)$ & $36(1)$ \\
\hline$C\left(25^{\prime}\right)$ & $4764(1)$ & $4915(1)$ & $3802(1)$ & $30(1)$ \\
\hline$C\left(26^{\prime}\right)$ & $3990(1)$ & $4912(1)$ & $4669(1)$ & $42(1)$ \\
\hline
\end{tabular}




\begin{tabular}{|c|c|c|c|c|}
\hline$C\left(27^{\prime}\right)$ & $3789(1)$ & $5718(1)$ & $3773(1)$ & $40(1)$ \\
\hline $\mathrm{Cl}\left(1^{\prime \prime}\right)$ & $6137(1)$ & $1170(1)$ & 1844(1) & $45(1)$ \\
\hline $\mathrm{O}(1 ")$ & 7101(1) & $1406(1)$ & $4219(1)$ & $35(1)$ \\
\hline $\mathrm{O}\left(2^{\prime \prime}\right)$ & $6461(1)$ & $-65(1)$ & $4832(1)$ & $32(1)$ \\
\hline O(3") & $9135(1)$ & $-46(1)$ & $4386(1)$ & $43(1)$ \\
\hline N(1") & $6554(1)$ & $-53(1)$ & $3330(1)$ & $33(1)$ \\
\hline N(2") & $7260(1)$ & $-1511(1)$ & $4841(1)$ & $46(1)$ \\
\hline N(3") & $6587(1)$ & 2401(2) & $7628(1)$ & $77(1)$ \\
\hline N(4") & $9080(1)$ & $-757(1)$ & $5237(1)$ & $37(1)$ \\
\hline C(1") & $6372(1)$ & $205(1)$ & 2743(1) & $35(1)$ \\
\hline C(2") & $6415(1)$ & $888(1)$ & 2613(1) & $35(1)$ \\
\hline C(3") & $6664(1)$ & 1341(1) & 3081(1) & $35(1)$ \\
\hline C(4") & $6843(1)$ & 1062(1) & $3686(1)$ & $32(1)$ \\
\hline C(5") & $6770(1)$ & $382(1)$ & $3790(1)$ & $30(1)$ \\
\hline C(6") & $6984(1)$ & 213(1) & $4486(1)$ & $30(1)$ \\
\hline C(7") & 7301(1) & $909(1)$ & $4728(1)$ & $32(1)$ \\
\hline C(8") & $8083(1)$ & 787(1) & 4731(1) & $31(1)$ \\
\hline C(9") & $8157(1)$ & $61(1)$ & $4982(1)$ & $30(1)$ \\
\hline C(10") & $7596(1)$ & $-285(1)$ & $4539(1)$ & $31(1)$ \\
\hline C(11") & $7402(1)$ & $-969(1)$ & $4721(1)$ & $36(1)$ \\
\hline C(12") & 7101(1) & $1204(1)$ & $5368(1)$ & $34(1)$ \\
\hline C(13") & $6968(1)$ & $817(1)$ & $5903(1)$ & $38(1)$ \\
\hline C(14") & $6842(1)$ & $1116(1)$ & $6494(1)$ & $45(1)$ \\
\hline C(15") & $6854(1)$ & 1812(1) & $6552(1)$ & $47(1)$ \\
\hline C(16") & 6999(1) & $2206(1)$ & $6021(1)$ & $45(1)$ \\
\hline C(17") & $7119(1)$ & 1907(1) & $5434(1)$ & $39(1)$ \\
\hline C(18") & $6706(1)$ & $2136(2)$ & $7158(1)$ & $59(1)$ \\
\hline C(19") & $8508(1)$ & $1328(1)$ & $5068(1)$ & $33(1)$ \\
\hline C(20") & $8716(1)$ & $1305(1)$ & $5725(1)$ & $38(1)$ \\
\hline C(21") & $9048(1)$ & 1851(1) & $6027(1)$ & $48(1)$ \\
\hline C(22") & $9176(1)$ & $2424(1)$ & $5671(1)$ & $55(1)$ \\
\hline C(23") & 8981(1) & $2447(1)$ & $5015(1)$ & $54(1)$ \\
\hline C(24") & $8653(1)$ & 1902(1) & $4716(1)$ & $41(1)$ \\
\hline C(25") & $8846(1)$ & $-252(1)$ & $4860(1)$ & $33(1)$ \\
\hline C(26") & $8746(1)$ & $-1026(1)$ & $5796(1)$ & $42(1)$ \\
\hline C(27") & $9705(1)$ & $-1106(1)$ & $5075(1)$ & $50(1)$ \\
\hline
\end{tabular}


Table 3. Bond lengths $[\AA]$ and angles $\left[{ }^{\circ}\right]$ for eFT1605.

\begin{tabular}{|c|c|c|c|}
\hline $\mathrm{Cl}(1)-\mathrm{C}(2)$ & $1.740(2)$ & $\mathrm{C}(13)-\mathrm{H}(13)$ & 0.9500 \\
\hline $\mathrm{O}(1)-\mathrm{C}(4)$ & $1.360(3)$ & $\mathrm{C}(13)-\mathrm{C}(14)$ & $1.384(3)$ \\
\hline $\mathrm{O}(1)-\mathrm{C}(7)$ & $1.473(2)$ & $\mathrm{C}(14)-\mathrm{H}(14)$ & 0.9500 \\
\hline $\mathrm{O}(2)-\mathrm{H}(2)$ & 0.8400 & $C(14)-C(15)$ & $1.390(3)$ \\
\hline $\mathrm{O}(2)-\mathrm{C}(6)$ & $1.401(2)$ & $C(15)-C(16)$ & $1.388(3)$ \\
\hline $\mathrm{O}(3)-\mathrm{C}(25)$ & $1.238(2)$ & $\mathrm{C}(15)-\mathrm{C}(18)$ & $1.444(3)$ \\
\hline $\mathrm{N}(1)-\mathrm{C}(1)$ & $1.342(3)$ & $\mathrm{C}(16)-\mathrm{H}(16)$ & 0.9500 \\
\hline $\mathrm{N}(1)-\mathrm{C}(5)$ & $1.329(3)$ & $C(16)-C(17)$ & $1.380(3)$ \\
\hline $\mathrm{N}(2)-\mathrm{C}(11)$ & $1.141(3)$ & $\mathrm{C}(17)-\mathrm{H}(17)$ & 0.9500 \\
\hline $\mathrm{N}(3)-\mathrm{C}(18)$ & $1.143(3)$ & $C(19)-C(20)$ & $1.385(3)$ \\
\hline $\mathrm{N}(4)-\mathrm{C}(25)$ & $1.333(2)$ & $C(19)-C(24)$ & $1.388(3)$ \\
\hline $\mathrm{N}(4)-\mathrm{C}(26)$ & $1.463(3)$ & $\mathrm{C}(20)-\mathrm{H}(20)$ & 0.9500 \\
\hline $\mathrm{N}(4)-\mathrm{C}(27)$ & $1.464(3)$ & $\mathrm{C}(20)-\mathrm{C}(21)$ & $1.382(4)$ \\
\hline $\mathrm{C}(1)-\mathrm{H}(1)$ & 0.9500 & $\mathrm{C}(21)-\mathrm{H}(21)$ & 0.9500 \\
\hline$C(1)-C(2)$ & $1.384(3)$ & $C(21)-C(22)$ & $1.379(4)$ \\
\hline $\mathrm{C}(2)-\mathrm{C}(3)$ & $1.378(3)$ & $\mathrm{C}(22)-\mathrm{H}(22)$ & 0.9500 \\
\hline $\mathrm{C}(3)-\mathrm{H}(3)$ & 0.9500 & $C(22)-C(23)$ & $1.367(4)$ \\
\hline $\mathrm{C}(3)-\mathrm{C}(4)$ & $1.386(3)$ & $\mathrm{C}(23)-\mathrm{H}(23)$ & 0.9500 \\
\hline$C(4)-C(5)$ & $1.377(3)$ & $C(23)-C(24)$ & $1.391(3)$ \\
\hline$C(5)-C(6)$ & $1.501(3)$ & $\mathrm{C}(24)-\mathrm{H}(24)$ & 0.9500 \\
\hline$C(6)-C(7)$ & $1.595(3)$ & $\mathrm{C}(26)-\mathrm{H}(26 \mathrm{~A})$ & 0.9800 \\
\hline$C(6)-C(10)$ & $1.551(3)$ & $\mathrm{C}(26)-\mathrm{H}(26 \mathrm{~B})$ & 0.9800 \\
\hline$C(7)-C(8)$ & $1.562(3)$ & $\mathrm{C}(26)-\mathrm{H}(26 \mathrm{C})$ & 0.9800 \\
\hline$C(7)-C(12)$ & $1.511(3)$ & $\mathrm{C}(27)-\mathrm{H}(27 \mathrm{~A})$ & 0.9800 \\
\hline $\mathrm{C}(8)-\mathrm{H}(8)$ & 1.0000 & $\mathrm{C}(27)-\mathrm{H}(27 \mathrm{~B})$ & 0.9800 \\
\hline $\mathrm{C}(8)-\mathrm{C}(9)$ & $1.535(3)$ & $\mathrm{C}(27)-\mathrm{H}(27 \mathrm{C})$ & 0.9800 \\
\hline $\mathrm{C}(8)-\mathrm{C}(19)$ & $1.506(3)$ & $\mathrm{Cl}\left(1^{\prime}\right)-\mathrm{C}\left(2^{\prime}\right)$ & $1.736(2)$ \\
\hline $\mathrm{C}(9)-\mathrm{H}(9)$ & 1.0000 & $\mathrm{O}\left(1^{\prime}\right)-\mathrm{C}\left(4^{\prime}\right)$ & $1.358(2)$ \\
\hline $\mathrm{C}(9)-\mathrm{C}(10)$ & $1.541(3)$ & $\mathrm{O}\left(1^{\prime}\right)-\mathrm{C}\left(7^{\prime}\right)$ & $1.465(2)$ \\
\hline $\mathrm{C}(9)-\mathrm{C}(25)$ & $1.527(3)$ & $\mathrm{O}\left(2^{\prime}\right)-\mathrm{H}\left(2^{\prime}\right)$ & 0.8400 \\
\hline $\mathrm{C}(10)-\mathrm{H}(10)$ & 1.0000 & $\mathrm{O}\left(2^{\prime}\right)-\mathrm{C}\left(6^{\prime}\right)$ & $1.402(2)$ \\
\hline$C(10)-C(11)$ & $1.471(3)$ & $\mathrm{O}\left(3^{\prime}\right)-\mathrm{C}\left(25^{\prime}\right)$ & $1.244(2)$ \\
\hline$C(12)-C(13)$ & $1.394(3)$ & $\mathrm{N}\left(1^{\prime}\right)-\mathrm{C}\left(1^{\prime}\right)$ & $1.348(3)$ \\
\hline $\mathrm{C}(12)-\mathrm{C}(17)$ & 1.391(3) & $\mathrm{N}\left(1^{\prime}\right)-\mathrm{C}\left(5^{\prime}\right)$ & $1.329(3)$ \\
\hline
\end{tabular}




\begin{tabular}{|c|c|c|c|}
\hline $\mathrm{N}\left(2^{\prime}\right)-\mathrm{C}\left(11^{\prime}\right)$ & $1.148(3)$ & $\mathrm{C}\left(19^{\prime}\right)-\mathrm{C}\left(24^{\prime}\right)$ & $1.392(3)$ \\
\hline $\mathrm{N}\left(3^{\prime}\right)-\mathrm{C}\left(18^{\prime}\right)$ & $1.142(3)$ & $\mathrm{C}\left(20^{\prime}\right)-\mathrm{H}\left(20^{\prime}\right)$ & 0.9500 \\
\hline $\mathrm{N}\left(4^{\prime}\right)-\mathrm{C}\left(25^{\prime}\right)$ & $1.330(3)$ & $\mathrm{C}\left(20^{\prime}\right)-\mathrm{C}\left(21^{\prime}\right)$ & $1.381(3)$ \\
\hline$N\left(4^{\prime}\right)-C\left(26^{\prime}\right)$ & $1.469(3)$ & $\mathrm{C}\left(21^{\prime}\right)-\mathrm{H}\left(21^{\prime}\right)$ & 0.9500 \\
\hline $\mathrm{N}\left(4^{\prime}\right)-\mathrm{C}\left(27^{\prime}\right)$ & $1.460(3)$ & $\mathrm{C}\left(21^{\prime}\right)-\mathrm{C}\left(22^{\prime}\right)$ & $1.380(4)$ \\
\hline $\mathrm{C}\left(1^{\prime}\right)-\mathrm{H}\left(1^{\prime}\right)$ & 0.9500 & $\mathrm{C}\left(22^{\prime}\right)-\mathrm{H}\left(22^{\prime}\right)$ & 0.9500 \\
\hline $\mathrm{C}\left(1^{\prime}\right)-\mathrm{C}\left(2^{\prime}\right)$ & $1.386(3)$ & $\mathrm{C}\left(22^{\prime}\right)-\mathrm{C}\left(23^{\prime}\right)$ & $1.382(4)$ \\
\hline $\mathrm{C}\left(2^{\prime}\right)-\mathrm{C}\left(3^{\prime}\right)$ & $1.384(3)$ & $\mathrm{C}\left(23^{\prime}\right)-\mathrm{H}\left(23^{\prime}\right)$ & 0.9500 \\
\hline $\mathrm{C}\left(3^{\prime}\right)-\mathrm{H}\left(3^{\prime}\right)$ & 0.9500 & $\mathrm{C}\left(23^{\prime}\right)-\mathrm{C}\left(24^{\prime}\right)$ & $1.387(3)$ \\
\hline$C\left(3^{\prime}\right)-C\left(4^{\prime}\right)$ & $1.382(3)$ & $\mathrm{C}\left(24^{\prime}\right)-\mathrm{H}\left(24^{\prime}\right)$ & 0.9500 \\
\hline$C\left(4^{\prime}\right)-C\left(5^{\prime}\right)$ & $1.380(3)$ & $\mathrm{C}\left(26^{\prime}\right)-\mathrm{H}(26 \mathrm{D})$ & 0.9800 \\
\hline$C\left(5^{\prime}\right)-C\left(6^{\prime}\right)$ & $1.517(3)$ & $\mathrm{C}\left(26^{\prime}\right)-\mathrm{H}(26 \mathrm{E})$ & 0.9800 \\
\hline$C\left(6^{\prime}\right)-C\left(7^{\prime}\right)$ & $1.563(3)$ & $\mathrm{C}\left(26^{\prime}\right)-\mathrm{H}(26 \mathrm{~F})$ & 0.9800 \\
\hline $\mathrm{C}\left(6^{\prime}\right)-\mathrm{C}\left(10^{\prime}\right)$ & $1.565(2)$ & $\mathrm{C}\left(27^{\prime}\right)-\mathrm{H}(27 \mathrm{D})$ & 0.9800 \\
\hline $\mathrm{C}\left(7^{\prime}\right)-\mathrm{C}\left(8^{\prime}\right)$ & $1.567(2)$ & $\mathrm{C}\left(27^{\prime}\right)-\mathrm{H}(27 \mathrm{E})$ & 0.9800 \\
\hline $\mathrm{C}\left(7^{\prime}\right)-\mathrm{C}\left(12^{\prime}\right)$ & $1.508(3)$ & $\mathrm{C}\left(27^{\prime}\right)-\mathrm{H}(27 \mathrm{~F})$ & 0.9800 \\
\hline $\mathrm{C}\left(8^{\prime}\right)-\mathrm{H}\left(8^{\prime}\right)$ & 1.0000 & $\mathrm{Cl}\left(1^{\prime \prime}\right)-\mathrm{C}\left(2^{\prime \prime}\right)$ & $1.734(2)$ \\
\hline $\mathrm{C}\left(8^{\prime}\right)-\mathrm{C}\left(9^{\prime}\right)$ & $1.528(3)$ & $\mathrm{O}(1 ")-\mathrm{C}(4 ")$ & $1.362(2)$ \\
\hline $\mathrm{C}\left(8^{\prime}\right)-\mathrm{C}\left(19^{\prime}\right)$ & $1.504(3)$ & $\mathrm{O}(1 ")-\mathrm{C}(7 ")$ & $1.475(2)$ \\
\hline $\mathrm{C}\left(9^{\prime}\right)-\mathrm{H}\left(9^{\prime}\right)$ & 1.0000 & $\mathrm{O}\left(2^{\prime \prime}\right)-\mathrm{H}\left(2^{\prime \prime}\right)$ & 0.8400 \\
\hline $\mathrm{C}\left(9^{\prime}\right)-\mathrm{C}\left(10^{\prime}\right)$ & $1.557(3)$ & $\mathrm{O}\left(2^{\prime \prime}\right)-\mathrm{C}(6 ")$ & $1.401(2)$ \\
\hline $\mathrm{C}\left(9^{\prime}\right)-\mathrm{C}\left(25^{\prime}\right)$ & $1.524(3)$ & $\mathrm{O}\left(3^{\prime \prime}\right)-\mathrm{C}\left(25^{\prime \prime}\right)$ & $1.233(2)$ \\
\hline $\mathrm{C}\left(10^{\prime}\right)-\mathrm{H}\left(10^{\prime}\right)$ & 1.0000 & N(1")-C(1") & $1.339(3)$ \\
\hline $\mathrm{C}\left(10^{\prime}\right)-\mathrm{C}\left(11^{\prime}\right)$ & $1.461(3)$ & N(1")-C(5") & $1.332(3)$ \\
\hline $\mathrm{C}\left(12^{\prime}\right)-\mathrm{C}\left(13^{\prime}\right)$ & $1.396(3)$ & N(2")-C(11") & $1.144(3)$ \\
\hline $\mathrm{C}\left(12^{\prime}\right)-\mathrm{C}\left(17^{\prime}\right)$ & $1.394(3)$ & N(3")-C(18") & $1.139(3)$ \\
\hline $\mathrm{C}\left(13^{\prime}\right)-\mathrm{H}\left(13^{\prime}\right)$ & 0.9500 & $\mathrm{~N}\left(4^{\prime \prime}\right)-\mathrm{C}\left(25^{\prime \prime}\right)$ & $1.333(3)$ \\
\hline $\mathrm{C}\left(13^{\prime}\right)-\mathrm{C}\left(14^{\prime}\right)$ & $1.384(3)$ & N(4")-C(26") & $1.463(3)$ \\
\hline $\mathrm{C}\left(14^{\prime}\right)-\mathrm{H}\left(14^{\prime}\right)$ & 0.9500 & N(4")-C(27") & $1.467(3)$ \\
\hline $\mathrm{C}\left(14^{\prime}\right)-\mathrm{C}\left(15^{\prime}\right)$ & $1.390(3)$ & $\mathrm{C}(1 ")-\mathrm{H}(1 ")$ & 0.9500 \\
\hline $\mathrm{C}\left(15^{\prime}\right)-\mathrm{C}\left(16^{\prime}\right)$ & $1.394(3)$ & C(1")-C(2") & $1.390(3)$ \\
\hline $\mathrm{C}\left(15^{\prime}\right)-\mathrm{C}\left(18^{\prime}\right)$ & $1.444(3)$ & $\mathrm{C}\left(2^{\prime \prime}\right)-\mathrm{C}\left(3^{\prime \prime}\right)$ & $1.383(3)$ \\
\hline $\mathrm{C}\left(16^{\prime}\right)-\mathrm{H}\left(16^{\prime}\right)$ & 0.9500 & C(3")-H(3") & 0.9500 \\
\hline $\mathrm{C}\left(16^{\prime}\right)-\mathrm{C}\left(17^{\prime}\right)$ & $1.379(3)$ & C(3")-C(4") & $1.387(3)$ \\
\hline $\mathrm{C}\left(17^{\prime}\right)-\mathrm{H}\left(17^{\prime}\right)$ & 0.9500 & $\mathrm{C}\left(4^{\prime \prime}\right)-\mathrm{C}\left(5^{\prime \prime}\right)$ & $1.381(3)$ \\
\hline $\mathrm{C}\left(19^{\prime}\right)-\mathrm{C}\left(20^{\prime}\right)$ & $1.389(3)$ & $\mathrm{C}\left(5^{\prime \prime}\right)-\mathrm{C}\left(6^{\prime \prime}\right)$ & $1.502(3)$ \\
\hline
\end{tabular}




\begin{tabular}{|c|c|c|c|}
\hline C(6")-C(7") & $1.586(3)$ & C(26")-H(26I) & 0.9800 \\
\hline C(6")-C(10") & $1.554(3)$ & $\mathrm{C}(27 ")-\mathrm{H}(27 \mathrm{G})$ & 0.9800 \\
\hline C(7")-C(8") & $1.553(3)$ & $\mathrm{C}(27 ")-\mathrm{H}(27 \mathrm{H})$ & 0.9800 \\
\hline C(7")-C(12") & $1.518(3)$ & C(27")-H(27I) & 0.9800 \\
\hline C(8")-H(8") & 1.0000 & & \\
\hline $\mathrm{C}\left(8^{\prime \prime}\right)-\mathrm{C}\left(9^{\prime \prime}\right)$ & $1.540(3)$ & $\mathrm{C}(4)-\mathrm{O}(1)-\mathrm{C}(7)$ & $108.10(14)$ \\
\hline C(8")-C(19") & $1.501(3)$ & $\mathrm{C}(6)-\mathrm{O}(2)-\mathrm{H}(2)$ & 109.5 \\
\hline $\mathrm{C}\left(9^{\prime \prime}\right)-\mathrm{H}\left(9^{\prime \prime}\right)$ & 1.0000 & $\mathrm{C}(5)-\mathrm{N}(1)-\mathrm{C}(1)$ & $115.81(17)$ \\
\hline C(9")-C(10") & $1.543(3)$ & $\mathrm{C}(25)-\mathrm{N}(4)-\mathrm{C}(26)$ & $120.08(17)$ \\
\hline $\mathrm{C}\left(9^{\prime \prime}\right)-\mathrm{C}\left(25^{\prime \prime}\right)$ & $1.523(3)$ & $\mathrm{C}(25)-\mathrm{N}(4)-\mathrm{C}(27)$ & $125.56(17)$ \\
\hline C(10")-H(10") & 1.0000 & $\mathrm{C}(26)-\mathrm{N}(4)-\mathrm{C}(27)$ & $114.35(16)$ \\
\hline C(10")-C(11") & $1.470(3)$ & $\mathrm{N}(1)-\mathrm{C}(1)-\mathrm{H}(1)$ & 118.9 \\
\hline C(12")-C(13") & $1.384(3)$ & $\mathrm{N}(1)-\mathrm{C}(1)-\mathrm{C}(2)$ & $122.2(2)$ \\
\hline C(12")-C(17") & $1.404(3)$ & $\mathrm{C}(2)-\mathrm{C}(1)-\mathrm{H}(1)$ & 118.9 \\
\hline C(13")-H(13") & 0.9500 & $\mathrm{C}(1)-\mathrm{C}(2)-\mathrm{Cl}(1)$ & $118.12(17)$ \\
\hline C(13")-C(14") & $1.392(3)$ & $\mathrm{C}(3)-\mathrm{C}(2)-\mathrm{Cl}(1)$ & $119.65(16)$ \\
\hline C(14")-H(14") & 0.9500 & $C(3)-C(2)-C(1)$ & $122.23(18)$ \\
\hline C(14")-C(15") & $1.390(4)$ & $\mathrm{C}(2)-\mathrm{C}(3)-\mathrm{H}(3)$ & 122.6 \\
\hline C(15")-C(16") & $1.391(4)$ & $C(2)-C(3)-C(4)$ & $114.87(18)$ \\
\hline C(15")-C(18") & $1.452(3)$ & $\mathrm{C}(4)-\mathrm{C}(3)-\mathrm{H}(3)$ & 122.6 \\
\hline C(16")-H(16") & 0.9500 & $\mathrm{O}(1)-\mathrm{C}(4)-\mathrm{C}(3)$ & $125.46(18)$ \\
\hline C(16")-C(17") & $1.382(3)$ & $\mathrm{O}(1)-\mathrm{C}(4)-\mathrm{C}(5)$ & $114.38(17)$ \\
\hline C(17")-H(17") & 0.9500 & $C(5)-C(4)-C(3)$ & $120.15(19)$ \\
\hline C(19")-C(20") & $1.387(3)$ & $\mathrm{N}(1)-\mathrm{C}(5)-\mathrm{C}(4)$ & $124.77(18)$ \\
\hline C(19")-C(24") & $1.392(3)$ & $\mathrm{N}(1)-\mathrm{C}(5)-\mathrm{C}(6)$ & $125.37(17)$ \\
\hline C(20")-H(20") & 0.9500 & $C(4)-C(5)-C(6)$ & $109.86(17)$ \\
\hline C(20")-C(21") & $1.391(3)$ & $\mathrm{O}(2)-\mathrm{C}(6)-\mathrm{C}(5)$ & $113.20(16)$ \\
\hline C(21")-H(21") & 0.9500 & $\mathrm{O}(2)-\mathrm{C}(6)-\mathrm{C}(7)$ & $117.18(16)$ \\
\hline C(21")-C(22") & $1.388(4)$ & $\mathrm{O}(2)-\mathrm{C}(6)-\mathrm{C}(10)$ & $108.33(14)$ \\
\hline C(22")-H(22") & 0.9500 & $C(5)-C(6)-C(7)$ & $101.16(14)$ \\
\hline C(22")-C(23") & $1.380(4)$ & $C(5)-C(6)-C(10)$ & $112.34(16)$ \\
\hline C(23")-H(23") & 0.9500 & $C(10)-C(6)-C(7)$ & $104.28(15)$ \\
\hline C(23")-C(24") & $1.385(3)$ & $\mathrm{O}(1)-\mathrm{C}(7)-\mathrm{C}(6)$ & $106.21(15)$ \\
\hline C(24")-H(24") & 0.9500 & $\mathrm{O}(1)-\mathrm{C}(7)-\mathrm{C}(8)$ & $107.99(14)$ \\
\hline $\mathrm{C}\left(26^{\prime \prime}\right)-\mathrm{H}(26 \mathrm{G})$ & 0.9800 & $\mathrm{O}(1)-\mathrm{C}(7)-\mathrm{C}(12)$ & $107.45(15)$ \\
\hline $\mathrm{C}\left(26^{\prime \prime}\right)-\mathrm{H}(26 \mathrm{H})$ & 0.9800 & $C(8)-C(7)-C(6)$ & $104.34(15)$ \\
\hline
\end{tabular}




\begin{tabular}{|c|c|c|c|}
\hline$C(12)-C(7)-C(6)$ & $115.97(15)$ & $\mathrm{C}(12)-\mathrm{C}(17)-\mathrm{H}(17)$ & 119.5 \\
\hline$C(12)-C(7)-C(8)$ & $114.38(16)$ & $C(16)-C(17)-C(12)$ & $121.02(19)$ \\
\hline $\mathrm{C}(7)-\mathrm{C}(8)-\mathrm{H}(8)$ & 106.5 & $\mathrm{C}(16)-\mathrm{C}(17)-\mathrm{H}(17)$ & 119.5 \\
\hline $\mathrm{C}(9)-\mathrm{C}(8)-\mathrm{C}(7)$ & $103.68(15)$ & $\mathrm{N}(3)-\mathrm{C}(18)-\mathrm{C}(15)$ & $176.9(3)$ \\
\hline $\mathrm{C}(9)-\mathrm{C}(8)-\mathrm{H}(8)$ & 106.5 & $C(20)-C(19)-C(8)$ & 119.11(19) \\
\hline $\mathrm{C}(19)-\mathrm{C}(8)-\mathrm{C}(7)$ & $115.42(16)$ & $\mathrm{C}(20)-\mathrm{C}(19)-\mathrm{C}(24)$ & $118.0(2)$ \\
\hline $\mathrm{C}(19)-\mathrm{C}(8)-\mathrm{H}(8)$ & 106.5 & $C(24)-C(19)-C(8)$ & $122.91(18)$ \\
\hline$C(19)-C(8)-C(9)$ & $117.55(16)$ & $\mathrm{C}(19)-\mathrm{C}(20)-\mathrm{H}(20)$ & 119.5 \\
\hline $\mathrm{C}(8)-\mathrm{C}(9)-\mathrm{H}(9)$ & 111.6 & $C(21)-C(20)-C(19)$ & $121.0(2)$ \\
\hline $\mathrm{C}(8)-\mathrm{C}(9)-\mathrm{C}(10)$ & $101.04(15)$ & $\mathrm{C}(21)-\mathrm{C}(20)-\mathrm{H}(20)$ & 119.5 \\
\hline $\mathrm{C}(10)-\mathrm{C}(9)-\mathrm{H}(9)$ & 111.6 & $\mathrm{C}(20)-\mathrm{C}(21)-\mathrm{H}(21)$ & 119.7 \\
\hline $\mathrm{C}(25)-\mathrm{C}(9)-\mathrm{C}(8)$ & $112.29(15)$ & $\mathrm{C}(22)-\mathrm{C}(21)-\mathrm{C}(20)$ & $120.5(2)$ \\
\hline $\mathrm{C}(25)-\mathrm{C}(9)-\mathrm{H}(9)$ & 111.6 & $\mathrm{C}(22)-\mathrm{C}(21)-\mathrm{H}(21)$ & 119.7 \\
\hline$C(25)-C(9)-C(10)$ & $108.33(15)$ & $\mathrm{C}(21)-\mathrm{C}(22)-\mathrm{H}(22)$ & 120.4 \\
\hline $\mathrm{C}(6)-\mathrm{C}(10)-\mathrm{H}(10)$ & 107.1 & $\mathrm{C}(23)-\mathrm{C}(22)-\mathrm{C}(21)$ & $119.2(2)$ \\
\hline $\mathrm{C}(9)-\mathrm{C}(10)-\mathrm{C}(6)$ & $106.36(15)$ & $\mathrm{C}(23)-\mathrm{C}(22)-\mathrm{H}(22)$ & 120.4 \\
\hline $\mathrm{C}(9)-\mathrm{C}(10)-\mathrm{H}(10)$ & 107.1 & $\mathrm{C}(22)-\mathrm{C}(23)-\mathrm{H}(23)$ & 119.7 \\
\hline$C(11)-C(10)-C(6)$ & $112.60(15)$ & $C(22)-C(23)-C(24)$ & $120.6(3)$ \\
\hline $\mathrm{C}(11)-\mathrm{C}(10)-\mathrm{C}(9)$ & $116.22(16)$ & $\mathrm{C}(24)-\mathrm{C}(23)-\mathrm{H}(23)$ & 119.7 \\
\hline $\mathrm{C}(11)-\mathrm{C}(10)-\mathrm{H}(10)$ & 107.1 & $\mathrm{C}(19)-\mathrm{C}(24)-\mathrm{C}(23)$ & $120.7(2)$ \\
\hline $\mathrm{N}(2)-\mathrm{C}(11)-\mathrm{C}(10)$ & $178.1(2)$ & $\mathrm{C}(19)-\mathrm{C}(24)-\mathrm{H}(24)$ & 119.7 \\
\hline$C(13)-C(12)-C(7)$ & 121.01(17) & $\mathrm{C}(23)-\mathrm{C}(24)-\mathrm{H}(24)$ & 119.7 \\
\hline$C(17)-C(12)-C(7)$ & $120.40(17)$ & $\mathrm{O}(3)-\mathrm{C}(25)-\mathrm{N}(4)$ & $123.40(18)$ \\
\hline $\mathrm{C}(17)-\mathrm{C}(12)-\mathrm{C}(13)$ & $118.58(19)$ & $\mathrm{O}(3)-\mathrm{C}(25)-\mathrm{C}(9)$ & $117.11(16)$ \\
\hline $\mathrm{C}(12)-\mathrm{C}(13)-\mathrm{H}(13)$ & 119.5 & $\mathrm{~N}(4)-\mathrm{C}(25)-\mathrm{C}(9)$ & $119.20(16)$ \\
\hline$C(14)-C(13)-C(12)$ & $120.91(18)$ & $\mathrm{N}(4)-\mathrm{C}(26)-\mathrm{H}(26 \mathrm{~A})$ & 109.5 \\
\hline $\mathrm{C}(14)-\mathrm{C}(13)-\mathrm{H}(13)$ & 119.5 & $\mathrm{~N}(4)-\mathrm{C}(26)-\mathrm{H}(26 \mathrm{~B})$ & 109.5 \\
\hline $\mathrm{C}(13)-\mathrm{C}(14)-\mathrm{H}(14)$ & 120.3 & $\mathrm{~N}(4)-\mathrm{C}(26)-\mathrm{H}(26 \mathrm{C})$ & 109.5 \\
\hline $\mathrm{C}(13)-\mathrm{C}(14)-\mathrm{C}(15)$ & $119.39(18)$ & $\mathrm{H}(26 \mathrm{~A})-\mathrm{C}(26)-\mathrm{H}(26 \mathrm{~B})$ & 109.5 \\
\hline $\mathrm{C}(15)-\mathrm{C}(14)-\mathrm{H}(14)$ & 120.3 & $\mathrm{H}(26 \mathrm{~A})-\mathrm{C}(26)-\mathrm{H}(26 \mathrm{C})$ & 109.5 \\
\hline $\mathrm{C}(14)-\mathrm{C}(15)-\mathrm{C}(18)$ & $121.45(19)$ & $H(26 B)-C(26)-H(26 C)$ & 109.5 \\
\hline$C(16)-C(15)-C(14)$ & $120.3(2)$ & $\mathrm{N}(4)-\mathrm{C}(27)-\mathrm{H}(27 \mathrm{~A})$ & 109.5 \\
\hline $\mathrm{C}(16)-\mathrm{C}(15)-\mathrm{C}(18)$ & $118.2(2)$ & $\mathrm{N}(4)-\mathrm{C}(27)-\mathrm{H}(27 \mathrm{~B})$ & 109.5 \\
\hline $\mathrm{C}(15)-\mathrm{C}(16)-\mathrm{H}(16)$ & 120.2 & $\mathrm{~N}(4)-\mathrm{C}(27)-\mathrm{H}(27 \mathrm{C})$ & 109.5 \\
\hline$C(17)-C(16)-C(15)$ & $119.62(19)$ & $\mathrm{H}(27 \mathrm{~A})-\mathrm{C}(27)-\mathrm{H}(27 \mathrm{~B})$ & 109.5 \\
\hline $\mathrm{C}(17)-\mathrm{C}(16)-\mathrm{H}(16)$ & 120.2 & $\mathrm{H}(27 \mathrm{~A})-\mathrm{C}(27)-\mathrm{H}(27 \mathrm{C})$ & 109.5 \\
\hline
\end{tabular}




\begin{tabular}{|c|c|c|c|}
\hline $\mathrm{H}(27 \mathrm{~B})-\mathrm{C}(27)-\mathrm{H}(27 \mathrm{C})$ & 109.5 & $\mathrm{C}\left(9^{\prime}\right)-\mathrm{C}\left(8^{\prime}\right)-\mathrm{H}\left(8^{\prime}\right)$ & 107.0 \\
\hline $\mathrm{C}\left(4^{\prime}\right)-\mathrm{O}\left(1^{\prime}\right)-\mathrm{C}\left(7^{\prime}\right)$ & $106.02(14)$ & $\mathrm{C}\left(19^{\prime}\right)-\mathrm{C}\left(8^{\prime}\right)-\mathrm{C}\left(7^{\prime}\right)$ & $114.42(15)$ \\
\hline $\mathrm{C}\left(6^{\prime}\right)-\mathrm{O}\left(2^{\prime}\right)-\mathrm{H}\left(2^{\prime}\right)$ & 109.5 & $\mathrm{C}\left(19^{\prime}\right)-\mathrm{C}\left(8^{\prime}\right)-\mathrm{H}\left(8^{\prime}\right)$ & 107.0 \\
\hline $\mathrm{C}\left(5^{\prime}\right)-\mathrm{N}\left(1^{\prime}\right)-\mathrm{C}\left(1^{\prime}\right)$ & $115.36(18)$ & $\mathrm{C}\left(19^{\prime}\right)-\mathrm{C}\left(8^{\prime}\right)-\mathrm{C}\left(9^{\prime}\right)$ & $119.05(15)$ \\
\hline $\mathrm{C}\left(25^{\prime}\right)-\mathrm{N}\left(4^{\prime}\right)-\mathrm{C}\left(26^{\prime}\right)$ & 119.66(17) & $\mathrm{C}\left(8^{\prime}\right)-\mathrm{C}\left(9^{\prime}\right)-\mathrm{H}\left(9^{\prime}\right)$ & 110.1 \\
\hline $\mathrm{C}\left(25^{\prime}\right)-\mathrm{N}\left(4^{\prime}\right)-\mathrm{C}\left(27^{\prime}\right)$ & $126.13(16)$ & $\mathrm{C}\left(8^{\prime}\right)-\mathrm{C}\left(9^{\prime}\right)-\mathrm{C}\left(10^{\prime}\right)$ & $101.64(14)$ \\
\hline $\mathrm{C}\left(27^{\prime}\right)-\mathrm{N}\left(4^{\prime}\right)-\mathrm{C}\left(26^{\prime}\right)$ & $114.05(16)$ & $\mathrm{C}\left(10^{\prime}\right)-\mathrm{C}\left(9^{\prime}\right)-\mathrm{H}\left(9^{\prime}\right)$ & 110.1 \\
\hline $\mathrm{N}\left(1^{\prime}\right)-\mathrm{C}\left(1^{\prime}\right)-\mathrm{H}\left(1^{\prime}\right)$ & 118.7 & $\mathrm{C}\left(25^{\prime}\right)-\mathrm{C}\left(9^{\prime}\right)-\mathrm{C}\left(8^{\prime}\right)$ & $114.07(16)$ \\
\hline $\mathrm{N}\left(1^{\prime}\right)-\mathrm{C}\left(1^{\prime}\right)-\mathrm{C}\left(2^{\prime}\right)$ & $122.58(19)$ & $\mathrm{C}\left(25^{\prime}\right)-\mathrm{C}\left(9^{\prime}\right)-\mathrm{H}\left(9^{\prime}\right)$ & 110.1 \\
\hline $\mathrm{C}\left(2^{\prime}\right)-\mathrm{C}\left(1^{\prime}\right)-\mathrm{H}\left(1^{\prime}\right)$ & 118.7 & $\mathrm{C}\left(25^{\prime}\right)-\mathrm{C}\left(9^{\prime}\right)-\mathrm{C}\left(10^{\prime}\right)$ & $110.60(15)$ \\
\hline $\mathrm{C}\left(1^{\prime}\right)-\mathrm{C}\left(2^{\prime}\right)-\mathrm{Cl}\left(1^{\prime}\right)$ & 118.61(16) & $\mathrm{C}\left(6^{\prime}\right)-\mathrm{C}\left(10^{\prime}\right)-\mathrm{H}\left(10^{\prime}\right)$ & 108.1 \\
\hline $\mathrm{C}\left(3^{\prime}\right)-\mathrm{C}\left(2^{\prime}\right)-\mathrm{Cl}\left(1^{\prime}\right)$ & $119.45(17)$ & $\mathrm{C}\left(9^{\prime}\right)-\mathrm{C}\left(10^{\prime}\right)-\mathrm{C}\left(6^{\prime}\right)$ & $105.87(15)$ \\
\hline $\mathrm{C}\left(3^{\prime}\right)-\mathrm{C}\left(2^{\prime}\right)-\mathrm{C}\left(1^{\prime}\right)$ & $121.88(18)$ & $\mathrm{C}\left(9^{\prime}\right)-\mathrm{C}\left(10^{\prime}\right)-\mathrm{H}\left(10^{\prime}\right)$ & 108.1 \\
\hline $\mathrm{C}\left(2^{\prime}\right)-\mathrm{C}\left(3^{\prime}\right)-\mathrm{H}\left(3^{\prime}\right)$ & 122.6 & $\mathrm{C}\left(11^{\prime}\right)-\mathrm{C}\left(10^{\prime}\right)-\mathrm{C}\left(6^{\prime}\right)$ & $112.64(16)$ \\
\hline $\mathrm{C}\left(4^{\prime}\right)-\mathrm{C}\left(3^{\prime}\right)-\mathrm{C}\left(2^{\prime}\right)$ & $114.83(19)$ & $\mathrm{C}\left(11^{\prime}\right)-\mathrm{C}\left(10^{\prime}\right)-\mathrm{C}\left(9^{\prime}\right)$ & $113.84(16)$ \\
\hline $\mathrm{C}\left(4^{\prime}\right)-\mathrm{C}\left(3^{\prime}\right)-\mathrm{H}\left(3^{\prime}\right)$ & 122.6 & $\mathrm{C}\left(11^{\prime}\right)-\mathrm{C}\left(10^{\prime}\right)-\mathrm{H}\left(10^{\prime}\right)$ & 108.1 \\
\hline $\mathrm{O}\left(1^{\prime}\right)-\mathrm{C}\left(4^{\prime}\right)-\mathrm{C}\left(3^{\prime}\right)$ & $125.47(18)$ & $\mathrm{N}\left(2^{\prime}\right)-\mathrm{C}\left(11^{\prime}\right)-\mathrm{C}\left(10^{\prime}\right)$ & $178.1(2)$ \\
\hline $\mathrm{O}\left(1^{\prime}\right)-\mathrm{C}\left(4^{\prime}\right)-\mathrm{C}\left(5^{\prime}\right)$ & $114.08(16)$ & $\mathrm{C}\left(13^{\prime}\right)-\mathrm{C}\left(12^{\prime}\right)-\mathrm{C}\left(7^{\prime}\right)$ & $120.76(17)$ \\
\hline $\mathrm{C}\left(5^{\prime}\right)-\mathrm{C}\left(4^{\prime}\right)-\mathrm{C}\left(3^{\prime}\right)$ & $120.46(18)$ & $\mathrm{C}\left(17^{\prime}\right)-\mathrm{C}\left(12^{\prime}\right)-\mathrm{C}\left(7^{\prime}\right)$ & $120.19(18)$ \\
\hline $\mathrm{N}\left(1^{\prime}\right)-\mathrm{C}\left(5^{\prime}\right)-\mathrm{C}\left(4^{\prime}\right)$ & $124.88(18)$ & $\mathrm{C}\left(17^{\prime}\right)-\mathrm{C}\left(12^{\prime}\right)-\mathrm{C}\left(13^{\prime}\right)$ & $118.62(18)$ \\
\hline $\mathrm{N}\left(1^{\prime}\right)-\mathrm{C}\left(5^{\prime}\right)-\mathrm{C}\left(6^{\prime}\right)$ & $126.05(18)$ & $\mathrm{C}\left(12^{\prime}\right)-\mathrm{C}\left(13^{\prime}\right)-\mathrm{H}\left(13^{\prime}\right)$ & 119.5 \\
\hline$C\left(4^{\prime}\right)-C\left(5^{\prime}\right)-C\left(6^{\prime}\right)$ & $108.96(17)$ & $\mathrm{C}\left(14^{\prime}\right)-\mathrm{C}\left(13^{\prime}\right)-\mathrm{C}\left(12^{\prime}\right)$ & $121.02(18)$ \\
\hline $\mathrm{O}\left(2^{\prime}\right)-\mathrm{C}\left(6^{\prime}\right)-\mathrm{C}\left(5^{\prime}\right)$ & $111.83(15)$ & $\mathrm{C}\left(14^{\prime}\right)-\mathrm{C}\left(13^{\prime}\right)-\mathrm{H}\left(13^{\prime}\right)$ & 119.5 \\
\hline $\mathrm{O}\left(2^{\prime}\right)-\mathrm{C}\left(6^{\prime}\right)-\mathrm{C}\left(7^{\prime}\right)$ & $117.52(15)$ & $\mathrm{C}\left(13^{\prime}\right)-\mathrm{C}\left(14^{\prime}\right)-\mathrm{H}\left(14^{\prime}\right)$ & 120.3 \\
\hline $\mathrm{O}\left(2^{\prime}\right)-\mathrm{C}\left(6^{\prime}\right)-\mathrm{C}\left(10^{\prime}\right)$ & $108.62(15)$ & $\mathrm{C}\left(13^{\prime}\right)-\mathrm{C}\left(14^{\prime}\right)-\mathrm{C}\left(15^{\prime}\right)$ & 119.32(19) \\
\hline$C\left(5^{\prime}\right)-\mathrm{C}\left(6^{\prime}\right)-\mathrm{C}\left(7^{\prime}\right)$ & $98.93(15)$ & $\mathrm{C}\left(15^{\prime}\right)-\mathrm{C}\left(14^{\prime}\right)-\mathrm{H}\left(14^{\prime}\right)$ & 120.3 \\
\hline $\mathrm{C}\left(5^{\prime}\right)-\mathrm{C}\left(6^{\prime}\right)-\mathrm{C}\left(10^{\prime}\right)$ & $114.64(15)$ & $\mathrm{C}\left(14^{\prime}\right)-\mathrm{C}\left(15^{\prime}\right)-\mathrm{C}\left(16^{\prime}\right)$ & $120.45(18)$ \\
\hline $\mathrm{C}\left(7^{\prime}\right)-\mathrm{C}\left(6^{\prime}\right)-\mathrm{C}\left(10^{\prime}\right)$ & $105.13(14)$ & $\mathrm{C}\left(14^{\prime}\right)-\mathrm{C}\left(15^{\prime}\right)-\mathrm{C}\left(18^{\prime}\right)$ & 119.07(19) \\
\hline $\mathrm{O}\left(1^{\prime}\right)-\mathrm{C}\left(7^{\prime}\right)-\mathrm{C}\left(6^{\prime}\right)$ & $107.19(14)$ & $\mathrm{C}\left(16^{\prime}\right)-\mathrm{C}\left(15^{\prime}\right)-\mathrm{C}\left(18^{\prime}\right)$ & $120.47(19)$ \\
\hline $\mathrm{O}\left(1^{\prime}\right)-\mathrm{C}\left(7^{\prime}\right)-\mathrm{C}\left(8^{\prime}\right)$ & $108.45(15)$ & $\mathrm{C}\left(15^{\prime}\right)-\mathrm{C}\left(16^{\prime}\right)-\mathrm{H}\left(16^{\prime}\right)$ & 120.2 \\
\hline $\mathrm{O}\left(1^{\prime}\right)-\mathrm{C}\left(7^{\prime}\right)-\mathrm{C}\left(12^{\prime}\right)$ & $108.19(14)$ & $\mathrm{C}\left(17^{\prime}\right)-\mathrm{C}\left(16^{\prime}\right)-\mathrm{C}\left(15^{\prime}\right)$ & $119.50(18)$ \\
\hline $\mathrm{C}\left(6^{\prime}\right)-\mathrm{C}\left(7^{\prime}\right)-\mathrm{C}\left(8^{\prime}\right)$ & $101.64(14)$ & $\mathrm{C}\left(17^{\prime}\right)-\mathrm{C}\left(16^{\prime}\right)-\mathrm{H}\left(16^{\prime}\right)$ & 120.2 \\
\hline $\mathrm{C}\left(12^{\prime}\right)-\mathrm{C}\left(7^{\prime}\right)-\mathrm{C}\left(6^{\prime}\right)$ & $118.39(17)$ & $\mathrm{C}\left(12^{\prime}\right)-\mathrm{C}\left(17^{\prime}\right)-\mathrm{H}\left(17^{\prime}\right)$ & 119.5 \\
\hline $\mathrm{C}\left(12^{\prime}\right)-\mathrm{C}\left(7^{\prime}\right)-\mathrm{C}\left(8^{\prime}\right)$ & $112.47(15)$ & $\mathrm{C}\left(16^{\prime}\right)-\mathrm{C}\left(17^{\prime}\right)-\mathrm{C}\left(12^{\prime}\right)$ & 121.02(19) \\
\hline $\mathrm{C}\left(7^{\prime}\right)-\mathrm{C}\left(8^{\prime}\right)-\mathrm{H}\left(8^{\prime}\right)$ & 107.0 & $\mathrm{C}\left(16^{\prime}\right)-\mathrm{C}\left(17^{\prime}\right)-\mathrm{H}\left(17^{\prime}\right)$ & 119.5 \\
\hline $\mathrm{C}\left(9^{\prime}\right)-\mathrm{C}\left(8^{\prime}\right)-\mathrm{C}\left(7^{\prime}\right)$ & $101.63(15)$ & $\mathrm{N}\left(3^{\prime}\right)-\mathrm{C}\left(18^{\prime}\right)-\mathrm{C}\left(15^{\prime}\right)$ & $178.6(2)$ \\
\hline
\end{tabular}




\begin{tabular}{|c|c|c|c|}
\hline $\mathrm{C}\left(20^{\prime}\right)-\mathrm{C}\left(19^{\prime}\right)-\mathrm{C}\left(8^{\prime}\right)$ & $122.67(18)$ & C(25")-N(4")-C(26") & $125.41(17)$ \\
\hline $\mathrm{C}\left(20^{\prime}\right)-\mathrm{C}\left(19^{\prime}\right)-\mathrm{C}\left(24^{\prime}\right)$ & $118.45(19)$ & $\mathrm{C}\left(25^{\prime \prime}\right)-\mathrm{N}\left(4^{\prime \prime}\right)-\mathrm{C}\left(27^{\prime \prime}\right)$ & $119.00(18)$ \\
\hline $\mathrm{C}\left(24^{\prime}\right)-\mathrm{C}\left(19^{\prime}\right)-\mathrm{C}\left(8^{\prime}\right)$ & $118.69(17)$ & $\mathrm{C}\left(26^{\prime \prime}\right)-\mathrm{N}\left(4^{\prime \prime}\right)-\mathrm{C}\left(27^{\prime \prime}\right)$ & $115.53(18)$ \\
\hline $\mathrm{C}\left(19^{\prime}\right)-\mathrm{C}\left(20^{\prime}\right)-\mathrm{H}\left(20^{\prime}\right)$ & 119.7 & N(1")-C(1")-H(1") & 118.9 \\
\hline $\mathrm{C}\left(21^{\prime}\right)-\mathrm{C}\left(20^{\prime}\right)-\mathrm{C}\left(19^{\prime}\right)$ & $120.6(2)$ & N(1")-C(1")-C(2") & $122.26(19)$ \\
\hline $\mathrm{C}\left(21^{\prime}\right)-\mathrm{C}\left(20^{\prime}\right)-\mathrm{H}\left(20^{\prime}\right)$ & 119.7 & $\mathrm{C}\left(2^{\prime \prime}\right)-\mathrm{C}(1 ")-\mathrm{H}\left(1^{\prime \prime}\right)$ & 118.9 \\
\hline $\mathrm{C}\left(20^{\prime}\right)-\mathrm{C}\left(21^{\prime}\right)-\mathrm{H}\left(21^{\prime}\right)$ & 119.8 & $\mathrm{C}\left(1^{\prime \prime}\right)-\mathrm{C}\left(2^{\prime \prime}\right)-\mathrm{Cl}\left(1^{\prime \prime}\right)$ & $118.31(16)$ \\
\hline $\mathrm{C}\left(22^{\prime}\right)-\mathrm{C}\left(21^{\prime}\right)-\mathrm{C}\left(20^{\prime}\right)$ & $120.5(2)$ & C(3")-C(2")-Cl(1") & $119.92(16)$ \\
\hline $\mathrm{C}\left(22^{\prime}\right)-\mathrm{C}\left(21^{\prime}\right)-\mathrm{H}\left(21^{\prime}\right)$ & 119.8 & C(3")-C(2")-C(1") & $121.76(19)$ \\
\hline $\mathrm{C}\left(21^{\prime}\right)-\mathrm{C}\left(22^{\prime}\right)-\mathrm{H}\left(22^{\prime}\right)$ & 120.1 & $\mathrm{C}\left(2^{\prime \prime}\right)-\mathrm{C}\left(3^{\prime \prime}\right)-\mathrm{H}\left(3^{\prime \prime}\right)$ & 122.6 \\
\hline $\mathrm{C}\left(21^{\prime}\right)-\mathrm{C}\left(22^{\prime}\right)-\mathrm{C}\left(23^{\prime}\right)$ & $119.8(2)$ & C(2")-C(3")-C(4") & $114.90(18)$ \\
\hline $\mathrm{C}\left(23^{\prime}\right)-\mathrm{C}\left(22^{\prime}\right)-\mathrm{H}\left(22^{\prime}\right)$ & 120.1 & $\mathrm{C}\left(4^{\prime \prime}\right)-\mathrm{C}\left(3^{\prime \prime}\right)-\mathrm{H}\left(3^{\prime \prime}\right)$ & 122.6 \\
\hline $\mathrm{C}\left(22^{\prime}\right)-\mathrm{C}\left(23^{\prime}\right)-\mathrm{H}\left(23^{\prime}\right)$ & 120.1 & $\mathrm{O}\left(1^{\prime \prime}\right)-\mathrm{C}\left(4^{\prime \prime}\right)-\mathrm{C}\left(3^{\prime \prime}\right)$ & $125.58(18)$ \\
\hline $\mathrm{C}\left(22^{\prime}\right)-\mathrm{C}\left(23^{\prime}\right)-\mathrm{C}\left(24^{\prime}\right)$ & $119.8(2)$ & $\mathrm{O}\left(1^{\prime \prime}\right)-\mathrm{C}\left(4^{\prime \prime}\right)-\mathrm{C}\left(5^{\prime \prime}\right)$ & $113.77(17)$ \\
\hline $\mathrm{C}\left(24^{\prime}\right)-\mathrm{C}\left(23^{\prime}\right)-\mathrm{H}\left(23^{\prime}\right)$ & 120.1 & C(5")-C(4")-C(3") & $120.65(19)$ \\
\hline $\mathrm{C}\left(19^{\prime}\right)-\mathrm{C}\left(24^{\prime}\right)-\mathrm{H}\left(24^{\prime}\right)$ & 119.5 & N(1")-C(5")-C(4") & $123.88(18)$ \\
\hline $\mathrm{C}\left(23^{\prime}\right)-\mathrm{C}\left(24^{\prime}\right)-\mathrm{C}\left(19^{\prime}\right)$ & $120.9(2)$ & $\mathrm{N}\left(1^{\prime \prime}\right)-\mathrm{C}\left(5^{\prime \prime}\right)-\mathrm{C}\left(6^{\prime \prime}\right)$ & $126.03(17)$ \\
\hline $\mathrm{C}\left(23^{\prime}\right)-\mathrm{C}\left(24^{\prime}\right)-\mathrm{H}\left(24^{\prime}\right)$ & 119.5 & C(4")-C(5")-C(6") & $110.08(17)$ \\
\hline $\mathrm{O}\left(3^{\prime}\right)-\mathrm{C}\left(25^{\prime}\right)-\mathrm{N}\left(4^{\prime}\right)$ & $123.35(17)$ & $\mathrm{O}\left(2^{\prime \prime}\right)-\mathrm{C}\left(6^{\prime \prime}\right)-\mathrm{C}\left(5^{\prime \prime}\right)$ & $114.04(16)$ \\
\hline $\mathrm{O}\left(3^{\prime}\right)-\mathrm{C}\left(25^{\prime}\right)-\mathrm{C}\left(9^{\prime}\right)$ & $116.98(16)$ & $\mathrm{O}\left(2^{\prime \prime}\right)-\mathrm{C}\left(6^{\prime \prime}\right)-\mathrm{C}\left(7^{\prime \prime}\right)$ & $117.96(15)$ \\
\hline $\mathrm{N}\left(4^{\prime}\right)-\mathrm{C}\left(25^{\prime}\right)-\mathrm{C}\left(9^{\prime}\right)$ & $119.65(17)$ & $\mathrm{O}\left(2^{\prime \prime}\right)-\mathrm{C}\left(6^{\prime \prime}\right)-\mathrm{C}\left(10^{\prime \prime}\right)$ & $107.40(15)$ \\
\hline $\mathrm{N}\left(4^{\prime}\right)-\mathrm{C}\left(26^{\prime}\right)-\mathrm{H}(26 \mathrm{D})$ & 109.5 & $\mathrm{C}\left(5^{\prime \prime}\right)-\mathrm{C}\left(6^{\prime \prime}\right)-\mathrm{C}\left(7^{\prime \prime}\right)$ & $100.53(15)$ \\
\hline $\mathrm{N}\left(4^{\prime}\right)-\mathrm{C}\left(26^{\prime}\right)-\mathrm{H}(26 \mathrm{E})$ & 109.5 & $\mathrm{C}\left(5^{\prime \prime}\right)-\mathrm{C}\left(6^{\prime \prime}\right)-\mathrm{C}\left(10^{\prime \prime}\right)$ & $111.96(15)$ \\
\hline $\mathrm{N}\left(4^{\prime}\right)-\mathrm{C}\left(26^{\prime}\right)-\mathrm{H}(26 \mathrm{~F})$ & 109.5 & $\mathrm{C}\left(10^{\prime \prime}\right)-\mathrm{C}\left(6^{\prime \prime}\right)-\mathrm{C}\left(7^{\prime \prime}\right)$ & $104.59(16)$ \\
\hline $\mathrm{H}(26 \mathrm{D})-\mathrm{C}\left(26^{\prime}\right)-\mathrm{H}(26 \mathrm{E})$ & 109.5 & $\mathrm{O}\left(1^{\prime \prime}\right)-\mathrm{C}\left(7^{\prime \prime}\right)-\mathrm{C}\left(6^{\prime \prime}\right)$ & $106.42(15)$ \\
\hline $\mathrm{H}(26 \mathrm{D})-\mathrm{C}\left(26^{\prime}\right)-\mathrm{H}(26 \mathrm{~F})$ & 109.5 & $\mathrm{O}\left(1^{\prime \prime}\right)-\mathrm{C}\left(7^{\prime \prime}\right)-\mathrm{C}\left(8^{\prime \prime}\right)$ & $108.72(15)$ \\
\hline $\mathrm{H}(26 \mathrm{E})-\mathrm{C}\left(26^{\prime}\right)-\mathrm{H}(26 \mathrm{~F})$ & 109.5 & $\mathrm{O}\left(1^{\prime \prime}\right)-\mathrm{C}\left(7^{\prime \prime}\right)-\mathrm{C}\left(12^{\prime \prime}\right)$ & $106.66(15)$ \\
\hline $\mathrm{N}\left(4^{\prime}\right)-\mathrm{C}\left(27^{\prime}\right)-\mathrm{H}(27 \mathrm{D})$ & 109.5 & C(8")-C(7")-C(6") & $103.27(15)$ \\
\hline $\mathrm{N}\left(4^{\prime}\right)-\mathrm{C}\left(27^{\prime}\right)-\mathrm{H}(27 \mathrm{E})$ & 109.5 & $\mathrm{C}\left(12^{\prime \prime}\right)-\mathrm{C}\left(7^{\prime \prime}\right)-\mathrm{C}\left(6^{\prime \prime}\right)$ & $119.60(16)$ \\
\hline $\mathrm{N}\left(4^{\prime}\right)-\mathrm{C}\left(27^{\prime}\right)-\mathrm{H}(27 \mathrm{~F})$ & 109.5 & C(12")-C(7")-C(8") & $111.73(16)$ \\
\hline $\mathrm{H}(27 \mathrm{D})-\mathrm{C}\left(27^{\prime}\right)-\mathrm{H}(27 \mathrm{E})$ & 109.5 & $\mathrm{C}\left(7^{\prime \prime}\right)-\mathrm{C}\left(8^{\prime \prime}\right)-\mathrm{H}\left(8^{\prime \prime}\right)$ & 106.9 \\
\hline $\mathrm{H}(27 \mathrm{D})-\mathrm{C}\left(27^{\prime}\right)-\mathrm{H}(27 \mathrm{~F})$ & 109.5 & C(9")-C(8")-C(7") & $102.58(15)$ \\
\hline $\mathrm{H}(27 \mathrm{E})-\mathrm{C}\left(27^{\prime}\right)-\mathrm{H}(27 \mathrm{~F})$ & 109.5 & $\mathrm{C}\left(9^{\prime \prime}\right)-\mathrm{C}\left(8^{\prime \prime}\right)-\mathrm{H}\left(8^{\prime \prime}\right)$ & 106.9 \\
\hline C(4")-O(1")-C(7") & $107.66(14)$ & C(19")-C(8")-C(7") & $113.97(16)$ \\
\hline C(6")-O(2")-H(2") & 109.5 & $\mathrm{C}\left(19^{\prime \prime}\right)-\mathrm{C}\left(8^{\prime \prime}\right)-\mathrm{H}\left(8^{\prime \prime}\right)$ & 106.9 \\
\hline $\mathrm{C}\left(5^{\prime \prime}\right)-\mathrm{N}\left(1^{\prime \prime}\right)-\mathrm{C}\left(1^{\prime \prime}\right)$ & $116.48(17)$ & C(19")-C(8")-C(9") & $118.76(17)$ \\
\hline
\end{tabular}




\begin{tabular}{|c|c|c|c|}
\hline $\mathrm{C}\left(8^{\prime \prime}\right)-\mathrm{C}\left(9^{\prime \prime}\right)-\mathrm{H}\left(9^{\prime \prime}\right)$ & 111.5 & $\mathrm{C}\left(19^{\prime \prime}\right)-\mathrm{C}\left(20^{\prime \prime}\right)-\mathrm{C}\left(21^{\prime \prime}\right)$ & $120.6(2)$ \\
\hline $\mathrm{C}\left(8^{\prime \prime}\right)-\mathrm{C}\left(9^{\prime \prime}\right)-\mathrm{C}\left(10^{\prime \prime}\right)$ & $99.98(15)$ & $\mathrm{C}\left(21^{\prime \prime}\right)-\mathrm{C}\left(20^{\prime \prime}\right)-\mathrm{H}\left(20^{\prime \prime}\right)$ & 119.7 \\
\hline C(10")-C(9")-H(9") & 111.5 & C(20")-C(21")-H(21") & 119.9 \\
\hline $\mathrm{C}\left(25^{\prime \prime}\right)-\mathrm{C}\left(9^{\prime \prime}\right)-\mathrm{C}\left(8^{\prime \prime}\right)$ & 113.09(16) & $\mathrm{C}\left(22^{\prime \prime}\right)-\mathrm{C}\left(21^{\prime \prime}\right)-\mathrm{C}\left(20^{\prime \prime}\right)$ & $120.1(2)$ \\
\hline $\mathrm{C}\left(25^{\prime \prime}\right)-\mathrm{C}\left(9^{\prime \prime}\right)-\mathrm{H}\left(9^{\prime \prime}\right)$ & 111.5 & $\mathrm{C}\left(22^{\prime \prime}\right)-\mathrm{C}\left(21^{\prime \prime}\right)-\mathrm{H}\left(21^{\prime \prime}\right)$ & 119.9 \\
\hline C(25")-C(9")-C(10") & $108.87(15)$ & $\mathrm{C}\left(21^{\prime \prime}\right)-\mathrm{C}\left(22^{\prime \prime}\right)-\mathrm{H}\left(22^{\prime \prime}\right)$ & 120.1 \\
\hline $\mathrm{C}\left(6^{\prime \prime}\right)-\mathrm{C}\left(10^{\prime \prime}\right)-\mathrm{H}\left(10^{\prime \prime}\right)$ & 106.8 & $\mathrm{C}(23 ")-\mathrm{C}\left(22^{\prime \prime}\right)-\mathrm{C}\left(21^{\prime \prime}\right)$ & $119.7(2)$ \\
\hline C(9")-C(10")-C(6") & $105.81(15)$ & $\mathrm{C}\left(23^{\prime \prime}\right)-\mathrm{C}\left(22^{\prime \prime}\right)-\mathrm{H}\left(22^{\prime \prime}\right)$ & 120.1 \\
\hline C(9")-C(10")-H(10") & 106.8 & $\mathrm{C}\left(22^{\prime \prime}\right)-\mathrm{C}\left(23^{\prime \prime}\right)-\mathrm{H}\left(23^{\prime \prime}\right)$ & 120.1 \\
\hline $\mathrm{C}\left(11^{\prime \prime}\right)-\mathrm{C}\left(10^{\prime \prime}\right)-\mathrm{C}\left(6^{\prime \prime}\right)$ & $113.44(17)$ & $\mathrm{C}\left(22^{\prime \prime}\right)-\mathrm{C}\left(23^{\prime \prime}\right)-\mathrm{C}\left(24^{\prime \prime}\right)$ & $119.9(2)$ \\
\hline $\mathrm{C}\left(11^{\prime \prime}\right)-\mathrm{C}\left(10^{\prime \prime}\right)-\mathrm{C}\left(9^{\prime \prime}\right)$ & $116.66(17)$ & $\mathrm{C}\left(24^{\prime \prime}\right)-\mathrm{C}\left(23^{\prime \prime}\right)-\mathrm{H}\left(23^{\prime \prime}\right)$ & 120.1 \\
\hline C(11")-C(10")-H(10") & 106.8 & C(19")-C(24")-H(24") & 119.4 \\
\hline N(2")-C(11")-C(10") & $177.4(2)$ & $\mathrm{C}\left(23^{\prime \prime}\right)-\mathrm{C}\left(24^{\prime \prime}\right)-\mathrm{C}\left(19^{\prime \prime}\right)$ & $121.2(2)$ \\
\hline $\mathrm{C}\left(13^{\prime \prime}\right)-\mathrm{C}\left(12^{\prime \prime}\right)-\mathrm{C}\left(7^{\prime \prime}\right)$ & $123.27(19)$ & $\mathrm{C}\left(23^{\prime \prime}\right)-\mathrm{C}\left(24^{\prime \prime}\right)-\mathrm{H}\left(24^{\prime \prime}\right)$ & 119.4 \\
\hline $\mathrm{C}\left(13^{\prime \prime}\right)-\mathrm{C}\left(12^{\prime \prime}\right)-\mathrm{C}\left(17^{\prime \prime}\right)$ & $118.82(19)$ & $\mathrm{O}\left(3^{\prime \prime}\right)-\mathrm{C}\left(25^{\prime \prime}\right)-\mathrm{N}\left(4^{\prime \prime}\right)$ & $123.60(18)$ \\
\hline $\mathrm{C}\left(17^{\prime \prime}\right)-\mathrm{C}\left(12^{\prime \prime}\right)-\mathrm{C}\left(7^{\prime \prime}\right)$ & 117.61(19) & $\mathrm{O}\left(3^{\prime \prime}\right)-\mathrm{C}\left(25^{\prime \prime}\right)-\mathrm{C}\left(9^{\prime \prime}\right)$ & $117.13(18)$ \\
\hline C(12")-C(13")-H(13") & 119.6 & N(4")-C(25")-C(9") & $119.10(17)$ \\
\hline $\mathrm{C}\left(12^{\prime \prime}\right)-\mathrm{C}\left(13^{\prime \prime}\right)-\mathrm{C}\left(14^{\prime \prime}\right)$ & $120.8(2)$ & $\mathrm{N}\left(4^{\prime \prime}\right)-\mathrm{C}\left(26^{\prime \prime}\right)-\mathrm{H}(26 \mathrm{G})$ & 109.5 \\
\hline C(14")-C(13")-H(13") & 119.6 & $\mathrm{~N}\left(4^{\prime \prime}\right)-\mathrm{C}\left(26^{\prime \prime}\right)-\mathrm{H}(26 \mathrm{H})$ & 109.5 \\
\hline C(13")-C(14")-H(14") & 120.1 & $\mathrm{~N}\left(4^{\prime \prime}\right)-\mathrm{C}\left(26^{\prime \prime}\right)-\mathrm{H}(26 \mathrm{I})$ & 109.5 \\
\hline $\mathrm{C}\left(15^{\prime \prime}\right)-\mathrm{C}\left(14^{\prime \prime}\right)-\mathrm{C}\left(13^{\prime \prime}\right)$ & $119.9(2)$ & $H(26 G)-C\left(26^{\prime \prime}\right)-H(26 H)$ & 109.5 \\
\hline C(15")-C(14")-H(14") & 120.1 & $H(26 G)-C(26 ")-H(26 I)$ & 109.5 \\
\hline $\mathrm{C}\left(14^{\prime \prime}\right)-\mathrm{C}\left(15^{\prime \prime}\right)-\mathrm{C}\left(16^{\prime \prime}\right)$ & $119.8(2)$ & $\mathrm{H}(26 \mathrm{H})-\mathrm{C}\left(26^{\prime \prime}\right)-\mathrm{H}(26 \mathrm{I})$ & 109.5 \\
\hline $\mathrm{C}\left(14^{\prime \prime}\right)-\mathrm{C}\left(15^{\prime \prime}\right)-\mathrm{C}\left(18^{\prime \prime}\right)$ & $120.9(3)$ & $\mathrm{N}\left(4^{\prime \prime}\right)-\mathrm{C}\left(27^{\prime \prime}\right)-\mathrm{H}(27 \mathrm{G})$ & 109.5 \\
\hline $\mathrm{C}\left(16^{\prime \prime}\right)-\mathrm{C}\left(15^{\prime \prime}\right)-\mathrm{C}\left(18^{\prime \prime}\right)$ & $119.3(2)$ & $\mathrm{N}\left(4^{\prime \prime}\right)-\mathrm{C}\left(27^{\prime \prime}\right)-\mathrm{H}(27 \mathrm{H})$ & 109.5 \\
\hline $\mathrm{C}\left(15^{\prime \prime}\right)-\mathrm{C}\left(16^{\prime \prime}\right)-\mathrm{H}\left(16^{\prime \prime}\right)$ & 120.0 & $\mathrm{~N}\left(4^{\prime \prime}\right)-\mathrm{C}\left(27^{\prime \prime}\right)-\mathrm{H}(27 \mathrm{I})$ & 109.5 \\
\hline $\mathrm{C}\left(17^{\prime \prime}\right)-\mathrm{C}\left(16^{\prime \prime}\right)-\mathrm{C}\left(15^{\prime \prime}\right)$ & $120.1(2)$ & $\mathrm{H}(27 \mathrm{G})-\mathrm{C}\left(27^{\prime \prime}\right)-\mathrm{H}(27 \mathrm{H})$ & 109.5 \\
\hline C(17")-C(16")-H(16") & 120.0 & $\mathrm{H}(27 \mathrm{G})-\mathrm{C}\left(27^{\prime \prime}\right)-\mathrm{H}(27 \mathrm{I})$ & 109.5 \\
\hline C(12")-C(17")-H(17") & 119.7 & $\mathrm{H}(27 \mathrm{H})-\mathrm{C}\left(27^{\prime \prime}\right)-\mathrm{H}(27 \mathrm{I})$ & 109.5 \\
\hline $\mathrm{C}\left(16^{\prime \prime}\right)-\mathrm{C}\left(17^{\prime \prime}\right)-\mathrm{C}\left(12^{\prime \prime}\right)$ & $120.6(2)$ & & \\
\hline C(16")-C(17")-H(17") & 119.7 & & \\
\hline N(3")-C(18")-C(15") & $178.8(3)$ & & \\
\hline $\mathrm{C}(20 ")-\mathrm{C}\left(19^{\prime \prime}\right)-\mathrm{C}\left(8^{\prime \prime}\right)$ & 123.01(19) & & \\
\hline $\mathrm{C}\left(20^{\prime \prime}\right)-\mathrm{C}\left(19^{\prime \prime}\right)-\mathrm{C}\left(24^{\prime \prime}\right)$ & $118.5(2)$ & & \\
\hline $\mathrm{C}\left(24^{\prime \prime}\right)-\mathrm{C}\left(19^{\prime \prime}\right)-\mathrm{C}\left(8^{\prime \prime}\right)$ & $118.30(19)$ & & \\
\hline $\mathrm{C}\left(19^{\prime \prime}\right)-\mathrm{C}\left(20^{\prime \prime}\right)-\mathrm{H}\left(20^{\prime \prime}\right)$ & 119.7 & & \\
\hline
\end{tabular}


Table 4. Anisotropic displacement parameters $\left(\AA^{2} \times 10^{3}\right)$ for eFT1605. The anisotropic displacement factor exponent takes the form: $-2 \pi^{2}\left[h^{2} a^{* 2} U^{11}+\ldots+2 h k a^{*} b^{*} U^{12}\right]$

\begin{tabular}{|c|c|c|c|c|c|c|}
\hline & $\mathrm{U}^{11}$ & $\mathrm{U}^{22}$ & $\mathrm{U}^{33}$ & $\mathrm{U}^{23}$ & $\mathrm{U}^{13}$ & $\mathrm{U}^{12}$ \\
\hline $\mathrm{Cl}(1)$ & $38(1)$ & $39(1)$ & $51(1)$ & $0(1)$ & $20(1)$ & $-2(1)$ \\
\hline $\mathrm{O}(1)$ & $35(1)$ & $42(1)$ & $23(1)$ & $2(1)$ & $7(1)$ & $-3(1)$ \\
\hline $\mathrm{O}(2)$ & $41(1)$ & $29(1)$ & $26(1)$ & $4(1)$ & $10(1)$ & $7(1)$ \\
\hline $\mathrm{O}(3)$ & $36(1)$ & $36(1)$ & $40(1)$ & $4(1)$ & $9(1)$ & $9(1)$ \\
\hline $\mathrm{N}(1)$ & $37(1)$ & $33(1)$ & $33(1)$ & $0(1)$ & $8(1)$ & $-2(1)$ \\
\hline $\mathrm{N}(2)$ & $42(1)$ & $42(1)$ & $30(1)$ & $0(1)$ & $6(1)$ & $6(1)$ \\
\hline $\mathrm{N}(3)$ & $64(2)$ & $82(2)$ & $47(1)$ & $13(1)$ & $2(1)$ & $30(1)$ \\
\hline $\mathrm{N}(4)$ & $32(1)$ & $32(1)$ & $32(1)$ & $6(1)$ & $4(1)$ & $0(1)$ \\
\hline $\mathrm{C}(1)$ & $34(1)$ & $36(1)$ & $40(1)$ & $-2(1)$ & $8(1)$ & $-2(1)$ \\
\hline$C(2)$ & $34(1)$ & $27(1)$ & $44(1)$ & $2(1)$ & $13(1)$ & $2(1)$ \\
\hline $\mathrm{C}(3)$ & $39(1)$ & $30(1)$ & $30(1)$ & $1(1)$ & $10(1)$ & $3(1)$ \\
\hline $\mathrm{C}(4)$ & $36(1)$ & $29(1)$ & $29(1)$ & $3(1)$ & $6(1)$ & $3(1)$ \\
\hline $\mathrm{C}(5)$ & $35(1)$ & $26(1)$ & $28(1)$ & $2(1)$ & $8(1)$ & 1(1) \\
\hline$C(6)$ & $33(1)$ & $30(1)$ & $27(1)$ & $3(1)$ & $6(1)$ & $4(1)$ \\
\hline $\mathrm{C}(7)$ & $34(1)$ & $34(1)$ & $23(1)$ & $3(1)$ & $9(1)$ & 1(1) \\
\hline $\mathrm{C}(8)$ & $30(1)$ & $33(1)$ & $25(1)$ & $2(1)$ & $7(1)$ & $4(1)$ \\
\hline $\mathrm{C}(9)$ & $27(1)$ & $31(1)$ & $27(1)$ & $4(1)$ & $5(1)$ & $4(1)$ \\
\hline$C(10)$ & $30(1)$ & $29(1)$ & $25(1)$ & $2(1)$ & $5(1)$ & 2(1) \\
\hline $\mathrm{C}(11)$ & $32(1)$ & $30(1)$ & $30(1)$ & $4(1)$ & $5(1)$ & $4(1)$ \\
\hline $\mathrm{C}(12)$ & $35(1)$ & $27(1)$ & $27(1)$ & $3(1)$ & $3(1)$ & $-1(1)$ \\
\hline $\mathrm{C}(13)$ & $41(1)$ & $32(1)$ & $25(1)$ & $0(1)$ & $3(1)$ & $-2(1)$ \\
\hline $\mathrm{C}(14)$ & $44(1)$ & $38(1)$ & $26(1)$ & $3(1)$ & $-4(1)$ & $-1(1)$ \\
\hline$C(15)$ & $38(1)$ & $35(1)$ & $34(1)$ & $2(1)$ & $-5(1)$ & 1(1) \\
\hline$C(16)$ & $37(1)$ & $42(1)$ & $35(1)$ & $4(1)$ & $6(1)$ & $5(1)$ \\
\hline $\mathrm{C}(17)$ & $39(1)$ & $39(1)$ & $27(1)$ & $7(1)$ & $4(1)$ & $5(1)$ \\
\hline $\mathrm{C}(18)$ & $50(2)$ & $52(1)$ & $33(1)$ & $6(1)$ & $-2(1)$ & $14(1)$ \\
\hline$C(19)$ & $38(1)$ & $30(1)$ & $31(1)$ & $3(1)$ & 1(1) & $4(1)$ \\
\hline $\mathrm{C}(20)$ & $49(2)$ & $55(1)$ & $37(1)$ & $-9(1)$ & $4(1)$ & $3(1)$ \\
\hline $\mathrm{C}(21)$ & $73(2)$ & $70(2)$ & $42(1)$ & $-21(1)$ & $-1(1)$ & $4(1)$ \\
\hline $\mathrm{C}(22)$ & $66(2)$ & $54(2)$ & $54(2)$ & $-7(1)$ & $-14(1)$ & $-8(1)$ \\
\hline$C(23)$ & $46(2)$ & $55(1)$ & $54(2)$ & $-1(1)$ & $-7(1)$ & $-10(1)$ \\
\hline $\mathrm{C}(24)$ & $42(1)$ & $50(1)$ & $39(1)$ & $-2(1)$ & $3(1)$ & $-6(1)$ \\
\hline
\end{tabular}




\begin{tabular}{|c|c|c|c|c|c|c|}
\hline$C(25)$ & $25(1)$ & $30(1)$ & $29(1)$ & 1(1) & 1(1) & $-2(1)$ \\
\hline$C(26)$ & $40(1)$ & $38(1)$ & $40(1)$ & $12(1)$ & 1(1) & 1(1) \\
\hline$C(27)$ & $49(1)$ & $38(1)$ & $35(1)$ & $3(1)$ & $18(1)$ & $-3(1)$ \\
\hline $\mathrm{Cl}\left(1^{\prime}\right)$ & $49(1)$ & $36(1)$ & $44(1)$ & $-2(1)$ & $12(1)$ & $10(1)$ \\
\hline $\mathrm{O}\left(1^{\prime}\right)$ & $28(1)$ & $30(1)$ & $33(1)$ & $-1(1)$ & $10(1)$ & $-1(1)$ \\
\hline $\mathrm{O}\left(2^{\prime}\right)$ & $30(1)$ & $38(1)$ & $26(1)$ & $0(1)$ & $7(1)$ & $0(1)$ \\
\hline $\mathrm{O}\left(3^{\prime}\right)$ & $44(1)$ & $34(1)$ & $33(1)$ & $6(1)$ & $15(1)$ & $4(1)$ \\
\hline $\mathrm{N}\left(1^{\prime}\right)$ & $39(1)$ & $32(1)$ & $32(1)$ & $-2(1)$ & $5(1)$ & $-2(1)$ \\
\hline $\mathrm{N}\left(2^{\prime}\right)$ & $37(1)$ & $58(1)$ & $42(1)$ & $-3(1)$ & $8(1)$ & $-5(1)$ \\
\hline $\mathrm{N}\left(3^{\prime}\right)$ & $48(1)$ & $35(1)$ & $71(1)$ & $-2(1)$ & $14(1)$ & $0(1)$ \\
\hline $\mathrm{N}\left(4^{\prime}\right)$ & $37(1)$ & $30(1)$ & $32(1)$ & $-1(1)$ & $13(1)$ & $-1(1)$ \\
\hline$C\left(1^{\prime}\right)$ & $46(1)$ & $30(1)$ & $34(1)$ & $-3(1)$ & $7(1)$ & $0(1)$ \\
\hline $\mathrm{C}\left(2^{\prime}\right)$ & $42(1)$ & $35(1)$ & $27(1)$ & $2(1)$ & $10(1)$ & $8(1)$ \\
\hline$C\left(3^{\prime}\right)$ & $32(1)$ & $35(1)$ & $28(1)$ & $3(1)$ & $8(1)$ & $3(1)$ \\
\hline$C\left(4^{\prime}\right)$ & $34(1)$ & $32(1)$ & $24(1)$ & $1(1)$ & $8(1)$ & $-2(1)$ \\
\hline$C\left(5^{\prime}\right)$ & $33(1)$ & $32(1)$ & $26(1)$ & $2(1)$ & $7(1)$ & $0(1)$ \\
\hline$C\left(6^{\prime}\right)$ & $29(1)$ & $31(1)$ & $27(1)$ & $0(1)$ & $7(1)$ & $-2(1)$ \\
\hline$C\left(7^{\prime}\right)$ & $26(1)$ & $32(1)$ & $29(1)$ & $-2(1)$ & $9(1)$ & $0(1)$ \\
\hline $\mathrm{C}\left(8^{\prime}\right)$ & $30(1)$ & $27(1)$ & $28(1)$ & $2(1)$ & $9(1)$ & $-1(1)$ \\
\hline $\mathrm{C}\left(9^{\prime}\right)$ & $32(1)$ & $28(1)$ & $28(1)$ & $1(1)$ & $7(1)$ & $-1(1)$ \\
\hline $\mathrm{C}\left(10^{\prime}\right)$ & $30(1)$ & $33(1)$ & $31(1)$ & $-1(1)$ & $9(1)$ & $-3(1)$ \\
\hline $\mathrm{C}\left(11^{\prime}\right)$ & $34(1)$ & $41(1)$ & $31(1)$ & $-3(1)$ & $10(1)$ & $-6(1)$ \\
\hline $\mathrm{C}\left(12^{\prime}\right)$ & $31(1)$ & $32(1)$ & $22(1)$ & 1(1) & $8(1)$ & $-1(1)$ \\
\hline $\mathrm{C}\left(13^{\prime}\right)$ & $30(1)$ & $33(1)$ & $26(1)$ & $0(1)$ & $6(1)$ & $-3(1)$ \\
\hline $\mathrm{C}\left(14^{\prime}\right)$ & $34(1)$ & $35(1)$ & $27(1)$ & $3(1)$ & $6(1)$ & $1(1)$ \\
\hline $\mathrm{C}\left(15^{\prime}\right)$ & $39(1)$ & $30(1)$ & $28(1)$ & $1(1)$ & $8(1)$ & $0(1)$ \\
\hline$C\left(16^{\prime}\right)$ & $32(1)$ & $36(1)$ & $32(1)$ & $1(1)$ & $7(1)$ & $-6(1)$ \\
\hline $\mathrm{C}\left(17^{\prime}\right)$ & $29(1)$ & $34(1)$ & $29(1)$ & $3(1)$ & $7(1)$ & $-1(1)$ \\
\hline $\mathrm{C}\left(18^{\prime}\right)$ & $37(1)$ & $37(1)$ & $42(1)$ & $-1(1)$ & $10(1)$ & $-2(1)$ \\
\hline $\mathrm{C}\left(19^{\prime}\right)$ & $31(1)$ & $33(1)$ & $24(1)$ & $3(1)$ & $8(1)$ & $-2(1)$ \\
\hline $\mathrm{C}\left(20^{\prime}\right)$ & $39(1)$ & $36(1)$ & $28(1)$ & $0(1)$ & $10(1)$ & $-1(1)$ \\
\hline $\mathrm{C}\left(21^{\prime}\right)$ & $57(2)$ & $37(1)$ & $29(1)$ & $-1(1)$ & 11(1) & $-7(1)$ \\
\hline$C\left(22^{\prime}\right)$ & $54(2)$ & $52(1)$ & $27(1)$ & $2(1)$ & $7(1)$ & $-20(1)$ \\
\hline $\mathrm{C}\left(23^{\prime}\right)$ & $39(1)$ & $57(1)$ & $35(1)$ & $7(1)$ & $1(1)$ & $-8(1)$ \\
\hline $\mathrm{C}\left(24^{\prime}\right)$ & $35(1)$ & $38(1)$ & $36(1)$ & $4(1)$ & $4(1)$ & $-1(1)$ \\
\hline$C\left(25^{\prime}\right)$ & $34(1)$ & $26(1)$ & $30(1)$ & $-1(1)$ & $8(1)$ & $-2(1)$ \\
\hline$C\left(26^{\prime}\right)$ & $54(1)$ & $37(1)$ & $38(1)$ & $0(1)$ & $24(1)$ & $-1(1)$ \\
\hline
\end{tabular}




\begin{tabular}{|c|c|c|c|c|c|c|}
\hline$C\left(27^{\prime}\right)$ & $41(1)$ & $42(1)$ & $37(1)$ & $-2(1)$ & $12(1)$ & $7(1)$ \\
\hline $\mathrm{Cl}(1 ")$ & $58(1)$ & $44(1)$ & $32(1)$ & $7(1)$ & $2(1)$ & 1(1) \\
\hline $\mathrm{O}(1 ")$ & $37(1)$ & $31(1)$ & $35(1)$ & $-1(1)$ & $-3(1)$ & $3(1)$ \\
\hline $\mathrm{O}\left(2^{\prime \prime}\right)$ & $30(1)$ & $33(1)$ & $33(1)$ & $3(1)$ & $9(1)$ & $5(1)$ \\
\hline $\mathrm{O}\left(3^{\prime \prime}\right)$ & $35(1)$ & $56(1)$ & $38(1)$ & $2(1)$ & $16(1)$ & $8(1)$ \\
\hline N(1") & $37(1)$ & $34(1)$ & $30(1)$ & $1(1)$ & $8(1)$ & $0(1)$ \\
\hline N(2") & $47(1)$ & $37(1)$ & $55(1)$ & $1(1)$ & $9(1)$ & $5(1)$ \\
\hline N(3") & $72(2)$ & $104(2)$ & $54(1)$ & $-32(1)$ & $-6(1)$ & $38(2)$ \\
\hline N(4") & $32(1)$ & $40(1)$ & $38(1)$ & $-3(1)$ & $3(1)$ & $10(1)$ \\
\hline $\mathrm{C}\left(1^{\prime \prime}\right)$ & $40(1)$ & $36(1)$ & $30(1)$ & $-1(1)$ & $6(1)$ & $-2(1)$ \\
\hline$C\left(2^{\prime \prime}\right)$ & $36(1)$ & $38(1)$ & $32(1)$ & $2(1)$ & $7(1)$ & $3(1)$ \\
\hline C(3") & $36(1)$ & $31(1)$ & $38(1)$ & $3(1)$ & $6(1)$ & $2(1)$ \\
\hline C(4") & $29(1)$ & $34(1)$ & $32(1)$ & $-3(1)$ & $4(1)$ & $2(1)$ \\
\hline C(5") & $26(1)$ & $33(1)$ & $31(1)$ & $1(1)$ & $6(1)$ & $2(1)$ \\
\hline C(6") & $28(1)$ & $33(1)$ & $29(1)$ & $-1(1)$ & $7(1)$ & $3(1)$ \\
\hline $\mathrm{C}\left(7^{\prime \prime}\right)$ & $30(1)$ & $33(1)$ & $32(1)$ & $1(1)$ & $2(1)$ & $5(1)$ \\
\hline C(8") & $28(1)$ & $36(1)$ & $30(1)$ & 1(1) & $6(1)$ & $2(1)$ \\
\hline C $\left(9^{\prime \prime}\right)$ & $29(1)$ & $34(1)$ & $26(1)$ & $0(1)$ & $8(1)$ & $6(1)$ \\
\hline $\mathrm{C}\left(10^{\prime \prime}\right)$ & $32(1)$ & $34(1)$ & $29(1)$ & $-2(1)$ & $7(1)$ & $6(1)$ \\
\hline$C\left(11^{\prime \prime}\right)$ & $32(1)$ & $38(1)$ & $39(1)$ & $-1(1)$ & $6(1)$ & $7(1)$ \\
\hline$C\left(12^{\prime \prime}\right)$ & $23(1)$ & $42(1)$ & $37(1)$ & $-7(1)$ & $2(1)$ & $7(1)$ \\
\hline$C\left(13^{\prime \prime}\right)$ & $30(1)$ & $48(1)$ & $36(1)$ & $-6(1)$ & $4(1)$ & $6(1)$ \\
\hline C(14") & $32(1)$ & $67(2)$ & $35(1)$ & $-7(1)$ & $3(1)$ & $8(1)$ \\
\hline$C\left(15^{\prime \prime}\right)$ & $30(1)$ & $66(2)$ & $44(1)$ & $-19(1)$ & $-2(1)$ & $13(1)$ \\
\hline$C\left(16^{\prime \prime}\right)$ & $31(1)$ & $48(1)$ & $54(1)$ & $-17(1)$ & $-5(1)$ & $12(1)$ \\
\hline$C\left(17^{\prime \prime}\right)$ & $27(1)$ & $43(1)$ & $46(1)$ & $-9(1)$ & $0(1)$ & $9(1)$ \\
\hline $\mathrm{C}\left(18^{\prime \prime}\right)$ & $47(2)$ & $81(2)$ & $48(1)$ & $-21(1)$ & $-4(1)$ & $25(1)$ \\
\hline C(19") & $27(1)$ & $38(1)$ & $36(1)$ & $-3(1)$ & $8(1)$ & $4(1)$ \\
\hline $\mathrm{C}\left(20^{\prime \prime}\right)$ & $31(1)$ & $46(1)$ & $39(1)$ & $-4(1)$ & $4(1)$ & $5(1)$ \\
\hline$C(21 ")$ & $34(1)$ & $59(2)$ & $50(1)$ & $-14(1)$ & $-3(1)$ & $6(1)$ \\
\hline$C\left(22^{\prime \prime}\right)$ & $43(2)$ & $52(1)$ & $70(2)$ & $-17(1)$ & $-1(1)$ & $-4(1)$ \\
\hline $\mathrm{C}\left(23^{\prime \prime}\right)$ & $50(2)$ & $42(1)$ & $71(2)$ & $-2(1)$ & $8(1)$ & $-6(1)$ \\
\hline $\mathrm{C}\left(24^{\prime \prime}\right)$ & $38(1)$ & $43(1)$ & $43(1)$ & $-1(1)$ & $8(1)$ & $-2(1)$ \\
\hline$C\left(25^{\prime \prime}\right)$ & $29(1)$ & $42(1)$ & $29(1)$ & $-6(1)$ & $6(1)$ & $6(1)$ \\
\hline$C\left(26^{\prime \prime}\right)$ & $43(1)$ & $44(1)$ & $40(1)$ & $6(1)$ & $-3(1)$ & $5(1)$ \\
\hline$C(27 ")$ & $38(1)$ & $51(1)$ & $62(2)$ & $-5(1)$ & $5(1)$ & $15(1)$ \\
\hline
\end{tabular}


Table 5. Hydrogen coordinates ( x 10 $)$ and isotropic displacement parameters $\left(\AA^{2} \times 10^{3}\right)$ for eFT1605.

\begin{tabular}{|c|c|c|c|c|}
\hline & $\mathrm{X}$ & $\mathrm{y}$ & $\mathrm{z}$ & $\mathrm{U}(\mathrm{eq})$ \\
\hline $\mathrm{H}(2)$ & 4998 & 3838 & 4705 & 48 \\
\hline $\mathrm{H}(1)$ & 7474 & 3756 & 4698 & 43 \\
\hline $\mathrm{H}(3)$ & 6605 & 3101 & 2970 & 39 \\
\hline $\mathrm{H}(8)$ & 5399 & 1702 & 4078 & 35 \\
\hline $\mathrm{H}(9)$ & 4388 & 1972 & 5000 & 34 \\
\hline $\mathrm{H}(10)$ & 5853 & 2167 & 5101 & 34 \\
\hline $\mathrm{H}(13)$ & 4430 & 3007 & 2823 & 39 \\
\hline $\mathrm{H}(14)$ & 3394 & 3521 & 2495 & 43 \\
\hline $\mathrm{H}(16)$ & 2840 & 3454 & 4356 & 45 \\
\hline $\mathrm{H}(17)$ & 3877 & 2946 & 4677 & 42 \\
\hline $\mathrm{H}(20)$ & 5083 & 1347 & 3002 & 56 \\
\hline $\mathrm{H}(21)$ & 4334 & 748 & 2299 & 74 \\
\hline $\mathrm{H}(22)$ & 3223 & 524 & 2578 & 71 \\
\hline $\mathrm{H}(23)$ & 2871 & 905 & 3565 & 63 \\
\hline $\mathrm{H}(24)$ & 3623 & 1496 & 4282 & 53 \\
\hline $\mathrm{H}(26 \mathrm{~A})$ & 4763 & 38 & 6086 & 59 \\
\hline $\mathrm{H}(26 \mathrm{~B})$ & 5300 & 487 & 6516 & 59 \\
\hline $\mathrm{H}(26 \mathrm{C})$ & 5500 & 194 & 5831 & 59 \\
\hline $\mathrm{H}(27 \mathrm{~A})$ & 4573 & 1606 & 6449 & 60 \\
\hline $\mathrm{H}(27 \mathrm{~B})$ & 4038 & 1011 & 6291 & 60 \\
\hline $\mathrm{H}(27 \mathrm{C})$ & 4073 & 1632 & 5802 & 60 \\
\hline $\mathrm{H}\left(2^{\prime}\right)$ & 5188 & 5005 & 1169 & 47 \\
\hline $\mathrm{H}\left(1^{\prime}\right)$ & 5854 & 2694 & 1580 & 44 \\
\hline $\mathrm{H}\left(3^{\prime}\right)$ & 7375 & 4057 & 1938 & 38 \\
\hline $\mathrm{H}\left(8^{\prime}\right)$ & 5920 & 4693 & 3196 & 33 \\
\hline $\mathrm{H}\left(9^{\prime}\right)$ & 4825 & 5657 & 3090 & 35 \\
\hline $\mathrm{H}\left(10^{\prime}\right)$ & 4758 & 4255 & 2737 & 37 \\
\hline H(13') & 4742 & 6091 & 2072 & 35 \\
\hline $\mathrm{H}\left(14^{\prime}\right)$ & 4665 & 7257 & 1993 & 38 \\
\hline $\mathrm{H}\left(16^{\prime}\right)$ & 6739 & 7381 & 2157 & 40 \\
\hline
\end{tabular}




\begin{tabular}{|c|c|c|c|c|}
\hline $\mathrm{H}\left(17^{\prime}\right)$ & 6807 & 6219 & 2262 & 37 \\
\hline $\mathrm{H}\left(20^{\prime}\right)$ & 5585 & 6411 & 3605 & 41 \\
\hline $\mathrm{H}\left(21^{\prime}\right)$ & 6358 & 7106 & 4186 & 49 \\
\hline $\mathrm{H}\left(22^{\prime}\right)$ & 7454 & 6725 & 4502 & 53 \\
\hline $\mathrm{H}\left(23^{\prime}\right)$ & 7776 & 5633 & 4242 & 52 \\
\hline $\mathrm{H}\left(24^{\prime}\right)$ & 7008 & 4939 & 3643 & 43 \\
\hline $\mathrm{H}(26 \mathrm{D})$ & 3582 & 4635 & 4572 & 63 \\
\hline $\mathrm{H}(26 \mathrm{E})$ & 3878 & 5281 & 4957 & 63 \\
\hline $\mathrm{H}(26 \mathrm{~F})$ & 4353 & 4636 & 4884 & 63 \\
\hline $\mathrm{H}(27 \mathrm{D})$ & 4027 & 5945 & 3432 & 60 \\
\hline $\mathrm{H}(27 \mathrm{E})$ & 3686 & 6043 & 4110 & 60 \\
\hline $\mathrm{H}(27 \mathrm{~F})$ & 3362 & 5522 & 3584 & 60 \\
\hline H(2") & 6141 & 213 & 4848 & 48 \\
\hline $\mathrm{H}\left(1^{\prime \prime}\right)$ & 6208 & -89 & 2405 & 42 \\
\hline $\mathrm{H}\left(3^{\prime \prime}\right)$ & 6708 & 1807 & 2994 & 42 \\
\hline H( $\left.8^{\prime \prime}\right)$ & 8202 & 783 & 4266 & 37 \\
\hline H(9") & 8058 & 29 & 5451 & 36 \\
\hline $\mathrm{H}\left(10^{\prime \prime}\right)$ & 7774 & -315 & 4096 & 38 \\
\hline H(13") & 6963 & 341 & 5866 & 45 \\
\hline H(14") & 6749 & 845 & 6857 & 54 \\
\hline $\mathrm{H}\left(16^{\prime \prime}\right)$ & 7014 & 2681 & 6061 & 54 \\
\hline H(17") & 7216 & 2178 & 5072 & 46 \\
\hline $\mathrm{H}\left(20^{\prime \prime}\right)$ & 8632 & 914 & 5970 & 46 \\
\hline $\mathrm{H}(21 ")$ & 9188 & 1831 & 6477 & 58 \\
\hline $\mathrm{H}\left(22^{\prime \prime}\right)$ & 9397 & 2799 & 5879 & 66 \\
\hline $\mathrm{H}\left(23^{\prime \prime}\right)$ & 9072 & 2836 & 4768 & 65 \\
\hline $\mathrm{H}(24 ")$ & 8524 & 1919 & 4264 & 50 \\
\hline $\mathrm{H}(26 \mathrm{G})$ & 8535 & -657 & 6026 & 64 \\
\hline $\mathrm{H}(26 \mathrm{H})$ & 9086 & -1251 & 6091 & 64 \\
\hline $\mathrm{H}(26 \mathrm{I})$ & 8393 & -1349 & 5644 & 64 \\
\hline $\mathrm{H}(27 \mathrm{G})$ & 9592 & -1567 & 4938 & 75 \\
\hline $\mathrm{H}(27 \mathrm{H})$ & 10030 & -1116 & 5458 & 75 \\
\hline $\mathrm{H}(27 \mathrm{I})$ & 9911 & -869 & 4720 & 75 \\
\hline
\end{tabular}


Table 6. Hydrogen bonds for eFT1605 [Å and $\left.{ }^{\circ}\right]$.

\begin{tabular}{lcccc}
\hline $\mathrm{D}-\mathrm{H} \ldots \mathrm{A}$ & $\mathrm{d}(\mathrm{D}-\mathrm{H})$ & $\mathrm{d}(\mathrm{H} \ldots \mathrm{A})$ & $\mathrm{d}(\mathrm{D} \ldots \mathrm{A})$ & $<(\mathrm{DHA})$ \\
\hline $\mathrm{O}(2)-\mathrm{H}(2) \ldots \mathrm{O}\left(3^{\prime}\right)$ & 0.84 & 1.82 & $2.6380(18)$ & 166.0 \\
$\mathrm{O}\left(2^{\prime}\right)-\mathrm{H}\left(2^{\prime}\right) \ldots \mathrm{O}\left(3^{\prime \prime}\right) \# 1$ & 0.84 & 1.81 & $2.6442(18)$ & 171.3 \\
$\mathrm{O}\left(2^{\prime \prime}\right)-\mathrm{H}\left(2^{\prime \prime}\right) \ldots \mathrm{O}(3)$ & 0.84 & 1.78 & $2.6198(19)$ & 177.3 \\
& & & & \\
\hline
\end{tabular}

Symmetry transformations used to generate equivalent atoms:

$\# 1-\mathrm{x}+3 / 2, \mathrm{y}+1 / 2,-\mathrm{z}+1 / 2$ 


\section{Biological assays}

\subsection{Cell proliferation assay}

MDA-MB-231 cells were cultured in DMEM media, 10\% FBS and 1x penicillin/streptomycin. Exponentially growing cells were seeded at 3000 cells per well in 96-well flat bottom white polystyrene plates from Thermofisher Scientific (Waltham, MA) and cultured overnight. The following day, compound was added in a 3-fold dilution series along with a DMSO control. The final DMSO concentration was $0.1 \%$. Cells were incubated for 72 hours at $37^{\circ} \mathrm{C}$ in a $\mathrm{CO}_{2}$ incubator. Baseline viability of untreated cells was measured on the day of treatment and proliferation was measured after 72 hours of treatment using CellTiter-Glo reagent from Promega (Madison, WI) according to manufactures instructions. Dose-response curves were plotted using Prism 6 software, and $\mathrm{EC}_{50}$ values were calculated using a 4 parameter, variable slope non-linear regression model.

Calculation of \% Inhibition:

$\%$ inhibition $=1-((($ cells + inhibitor $)-$ baseline $) /(($ cells + DMSO $)-$ baseline $))) X 100$.

\subsection{In vitro translation reporter assay}

c-MYC or Tubulin 5'-UTRs were cloned into the luciferase reporter vector pGL3 (Promega Corp., Madison, WI) at Genewiz (San Diego). DNA templates were PCR amplified using Phusion Highfidelity PCR Master Mix (New England Biolabs, Ipswich, MA) from the reporter vector that incorporates the T7 promoter sequence for subsequent RNA transcription. Reactions were purified using the Qiagen PCR clean up kit and eluted in 50uL water. RNA was transcribed from the DNA templates using the mMESSAGE mMACHINE T7 Ultra kit (Ambion ThermoFisher, Waltham, MA). RNA was purified with the RNA Mega Clear Kit and eluted with $30 \mu \mathrm{L}$ elution buffer (Ambion ThermoFisher, Waltham, MA). Using the manufacturer's instructions, RNA was transiently transfected into exponentially growing MDA-MB-231 cancer cells seeded into 96-well plates using the TransIT mRNA transfection kit (Mirus Bio, Madison, WI). The transfected cells were treated with various concentrations of compounds or control (DMSO) for 4 hours at $37^{\circ} \mathrm{C}$. 
Cells were rinsed with $100 \mu \mathrm{L}$ PBS and lysed using $50 \mu \mathrm{L}$ 1x passive lysis buffer (Promega Corp., Madison, WI). After shaking for 20 minutes at room temperature, $100 \mu \mathrm{L}$ luciferase assay reagent (Promega Corp., Madison, WI) was added and luminescence was quantitated using a Victor (Perkin Elmer) plate reader. The data were fitted using GraphPad Prism (GraphPad Software, La Jolla, CA) and a four-parameter dose response equation.

See Figure S1 and Table S1 for data obtained for compounds 1, 2, 24, 25 and CHX.
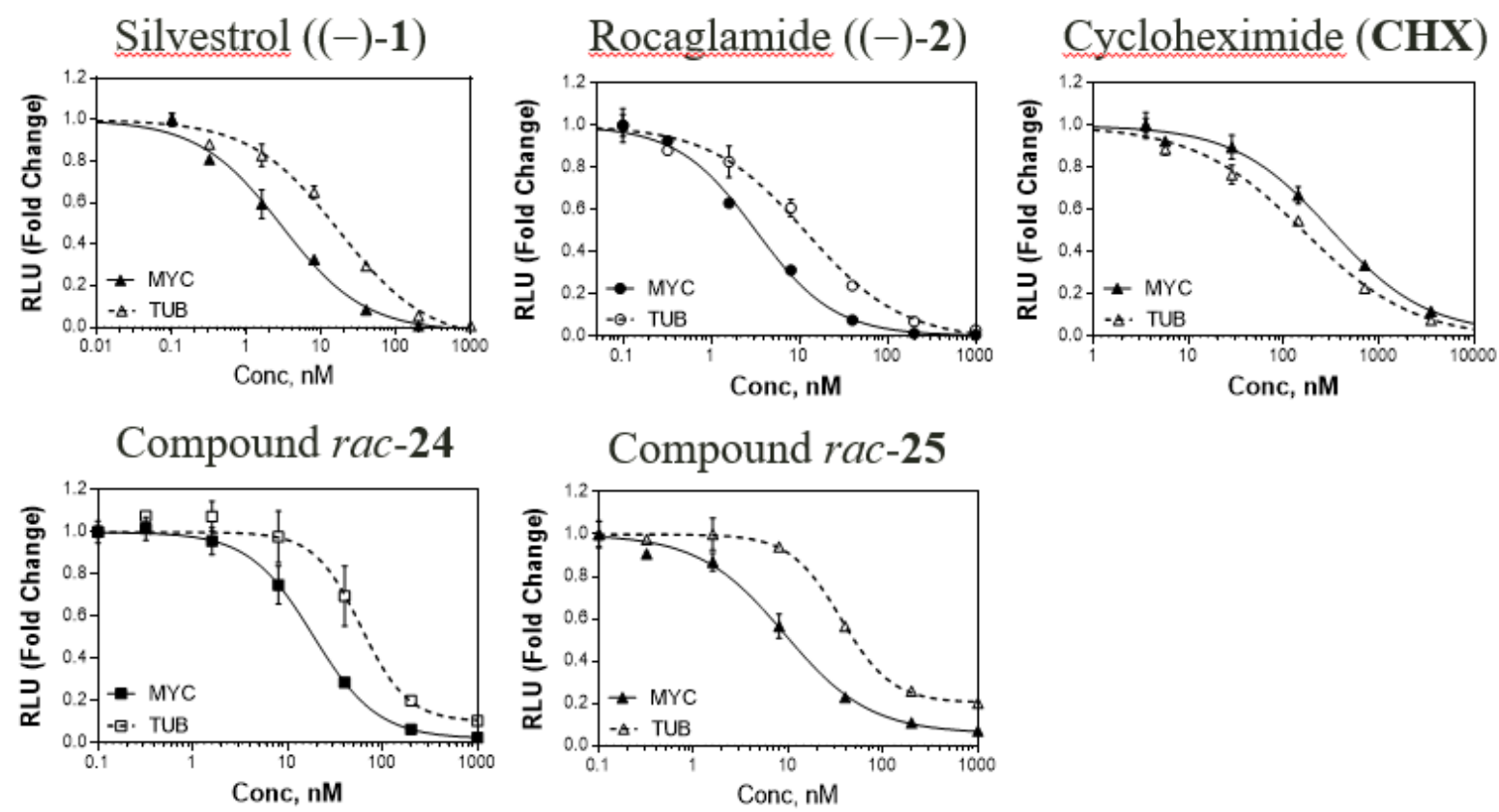

\section{Compound rac-25}

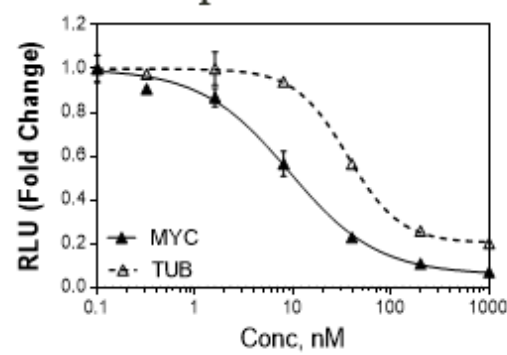

Figure S1: Cell based translation reporter assay. Relative luciferase expressed in the in vitro luciferase reporter assay containing either the highly structured 5'-UTR of c-MYC or the short 5'UTR of tubulin. The luciferase reporter gene constructs were transiently transfected into the MDAMB-231 cell line and treated with increasing concentrations of compound for 4 hours in triplicate. Data were fitted to a four-parameter dose response curve. 
Table S1: Cell based translation reporter assay results for compounds 1, 2, 24, 25 and cycloheximide (CHX).

\begin{tabular}{ccc} 
Compound & MYC IC $_{\mathbf{5 0}}(\mathbf{n M})$ & TUB IC $_{\mathbf{5 0}}(\mathbf{n M})$ \\
\hline$(-)-1$ & 3 & 13 \\
$(-)-2$ & 3 & 12 \\
$\boldsymbol{r a c - 2 4}$ & 19 & 59 \\
$\boldsymbol{r a c - 2 5}$ & 9 & 36 \\
$\mathbf{C H X}$ & 311 & 165 \\
\hline
\end{tabular}

